



\section{PARAMETRIC MODELLING \& DIGITAL MANUFACTURING FOR BETTER BRA FIT}





\title{
Parametric modelling \& digital manufacturing for better bra fit
}

\section{Ariya Xuxu Amoozegar-Montero}

\author{
A thesis submitted to the Victoria University of Wellington in fulfilment \\ of the requirements for the degree of Master of Design Innovation \\ Victoria University of Wellington (2016)
}



This thesis is dedicated to all the women of the world who have endured generations of ill fitted designed bras. 

I would like to give my sincere thanks to the following people for their support and encouragement,

Dr. Edgar Rodriguez Ramirez

$$
\text { Papi }
$$

Mama \& Aida

Ben

My Wellington hermanas,

Esther, Beanie, Kelly, Char, Lauren, Emma \& Jade

\author{
Gordon Fraser \\ \& Peter Heslop, AUT Textile \& Design Lab
}

Miriam McCreanor, Massey University

Level 2 studio peers,

$\&$ all the staff at the

Faculty of Architecture \& Design, VUW 



\section{ABSTR ACT}

This research focuses on developing an overall system for a more accurate bra design and fit. The bra, consequently, becomes part of a life-long service system that is able to adapt to both physical and personal desires of different types of bra wearers. Due to the symbiotic relationship between bra fit and bra design, a parametric system has also been designed in order to digitize the measuring process. This process explores the possibility of bringing the bra into a product-servicesystem framework. Individuality will not only be in the form of taste, preference, attitude, and aesthetics, but also through one's inherent breast shape, structure, contours, and asymmetry.

Under this paradigm, the designed bra system should not operate independently of its intended users and must always connect back to individual needs at every stage. For instance, to understand what makes a good fit cannot merely rely on the knowledge gained from literature or industry standards. To understand what makes a good fit, bra wearers themselves must be invited to offer their input. Therefore, this research could also be considered part of a human-centered design methodology. As such, this work was conducted as a mixed-methods approach with a combination of qualitative and quantitative processes. Unlike traditional bra fitting methods, this system utilizes advanced technologies and digital manufacturing techniques. Using technologies such as 3D scanning, to consider additional factors that are not currently considered, such as breast outline, shape, and asymmetry. This research also investigated the role of $3 \mathrm{D}$ knitting to fill the gap of customization and individualization at a mass production level, as well as serving as an innovative approach to respond to bra fitting and design issues. The data accumulated helped influence unique bra designs in order to insure better individual bra fit, whilst also acting as a medium to give the bra wearer a better understanding of their breast measurements, and how the bra fits on them.

Finally, the thesis compares, contrasts and identifies gaps within current methods for bra fitting and bra design, a parametric modelled measuring system, and final designed bra prototypes. Overall, it resulted in an effective parametric measuring system, which is able to adapt to individual 3D scans. Additionally, it was suggested that the 3D knitted prototype bra provided improved alternatives to a traditional bra, such as a seamless knitted underwire, which provides a more comfortable and flexible fit. Furthermore, our hope is to increase the consumer engagement and awareness about their own breasts and individual bra fit. After all, if the necessary information can be provided to the consumer regarding how to achieve a good bra fit, then this could help the consumer's ability for self-selecting better bra fit and enhance the satisfaction they receive from all bras. Therefore, the research might have a broad positive impact on a large size of the population. 
CONTENT

$\vec{o}$ 
Figure list.

01 Introduction

$14-21$

02 Background.

$.22-57$

03 Bra System $.58-67$

04 Methodology. $.68-75$

05 Bra Experience. $.76-103$

06 Design Themes. $.104-109$

07 Breast Data Collection $110-131$

08 Bra Design Development: 3D Knitting Exploration $132-159$

09 Bra Design Development: Utilization of Measurements. $160-189$

10 Results. $190-209$

11 Discussion \& Conclusion. $.210-215$

References. $216-221$

Appendix 1 $.222-231$

Appendix II. $.232-239$

Appendix III. $.240-243$ 


\section{FIGURE LIST}

\section{Chapter 01}

1.1. Anatomy of the bra.

\section{Chapter 02}

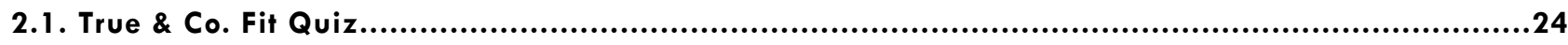

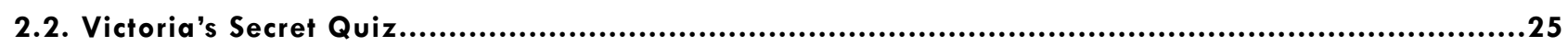

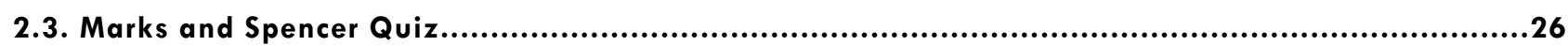

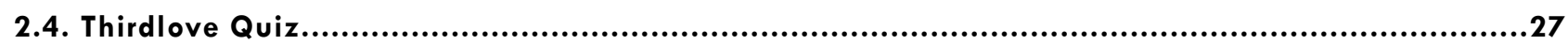

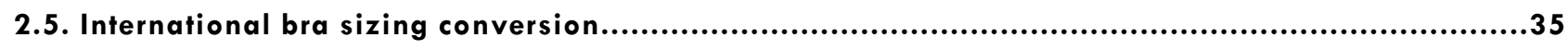

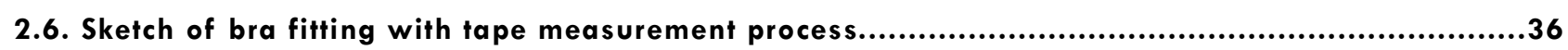

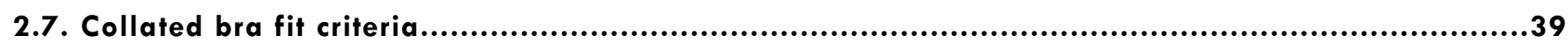

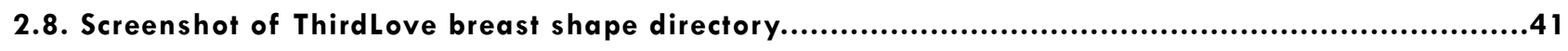

2.9. Sketch of breast landmarking points with breast outline circled..........................................44

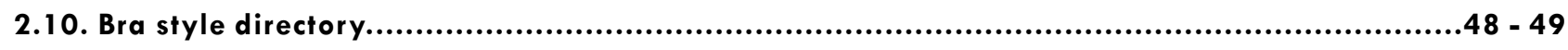

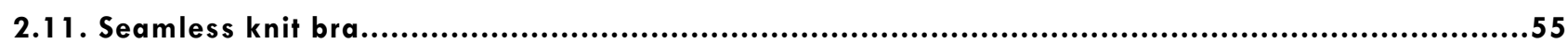

\section{Chapter 03}

3.1. Traditional and alternative systems................................................................60 - 61

3.2. Designer and consumer roles.................................................................................63

3.3.0. - 3.3.2. Images of a Shima Seiki 3D knitting machine..............................................65

3.4. Initial criteria for breast data collection......................................................................66

3.5. Initial criteria for bra design development: 3D knitting exploration......................................67

\section{Chapter 04}

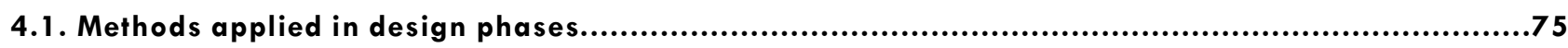

\section{Chapter 05}

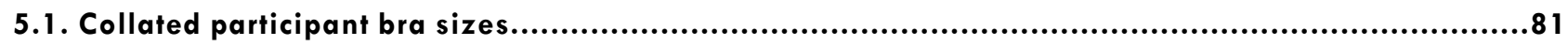

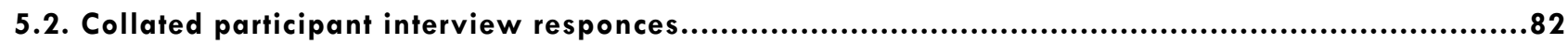

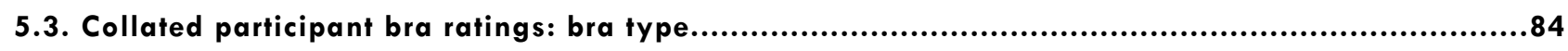

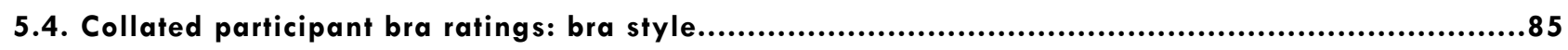

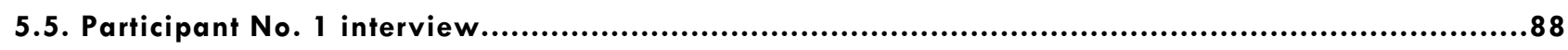

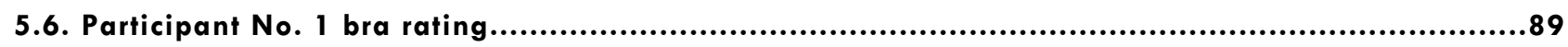

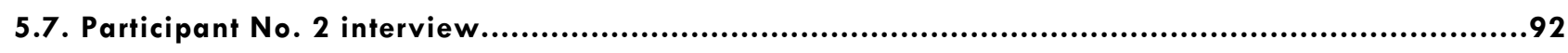

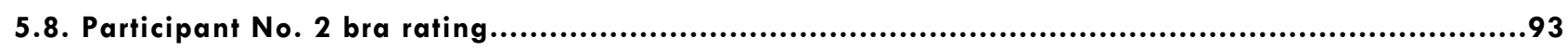

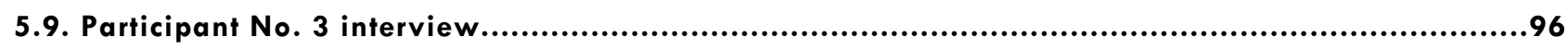

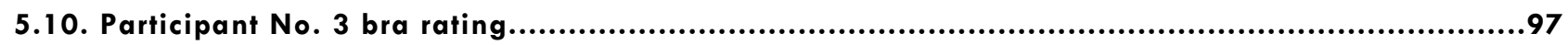

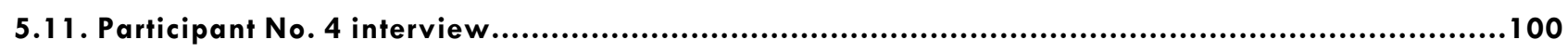

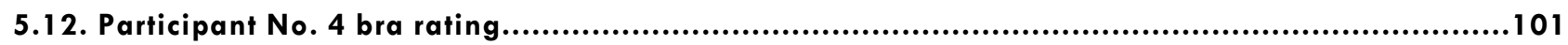




\section{Chapter 07}

7.1. 3D scans of participants, top, front and side view................................................ 15

7.2. Landmarking points explained.............................................................................116

7.3. Additional landmarking points for bra design....................................................... 17

7.4. Sketch of all design landmarking points on torso................................................. 18

7.5. 3D scans with landmarking points and breast outline highlighted............................120 - 121

7.6.0 - 7.6.3. Automation of points in Grasshopper series......................................122 - 125

7.7. Sketch of landmarking points utilized for underline and bust line comparisons.................... 27

\section{Chapter 08}

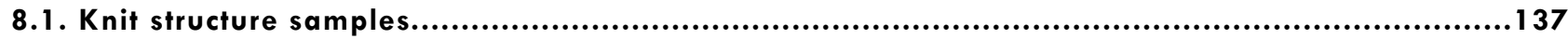

8.2. Screenshot of Shima Seiki digital knit design programming..............................................139

8.3.0. - 8.3.2. Images of "initial yarn selection" iterations.......................................142 - 143

8.4.0. - 8.4.1. Images of "addition of straps" iterations............................................144 - 145

8.5.0. - 8.5.1. Images of "yarn and structure development" iterations.............................146 - 147

8.6.0. - 8.6.1. Images of "incorporating polytex" iterations..............................................148

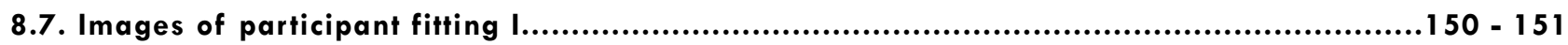

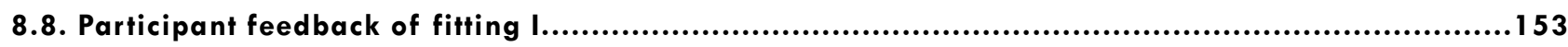

8.9. Sketches of potential bra designs............................................................... 154 - 155

8.10.0. - 8.10.1. Images of "vertical cup seam" iterations.......................................................157

\section{Chapter 09}

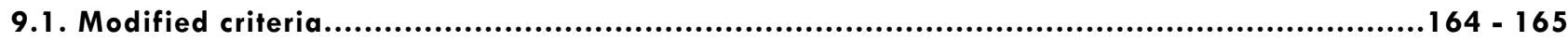

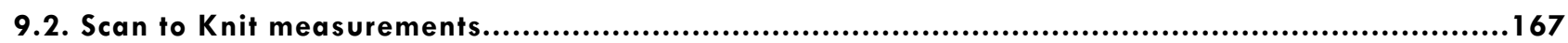

9.3.0 - 9.3.4. Scan to Knit Grasshopper series.............................................................169 - 173

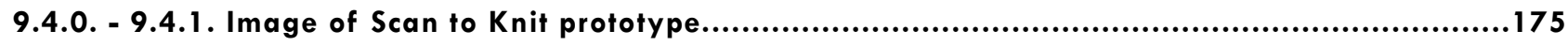

9.5. Scan to Sew measurements............................................................................... 176

9.6.0. - 9.6.4. Scan to Sew Grasshopper series.......................................................179-183

9.7.0. - 9.7.1. Image of Scan to Sew prototype..................................................................... 185

9.8. Comparing Scan to Knit and Scan to Sew measurements................................................186

9.9. Comparing Scan to Knit and Scan to Sew.................................................................... 187

\section{Chapter 10}

10.1 Participant fitting II Scan to Knit prototype.......................................................... 194

10.2. Participant fitting II Scan to Knit prototype................................................................195

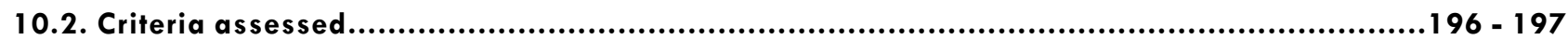

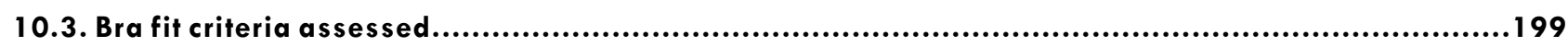

10.4.0. - 10.4.5. Final images of Scan to Knit bra......................................................202 - 205

10.5.0. - 10.5.6. Final Images of Scan to Sew bra...........................................................206 - 209 

C H A P T E R 01

INTRODUCTION 
$\vec{a}$ 
It has been more than a hundred years since Mary Phelps Jacob received a patent for the first bra design that is commonly recognized as the basis for all modern bras. About the same time, the First World War accelerated the role of women in society, putting many women to work in factories and uniforms for the first time. The war may have also helped influence social mores, freeing women from constraining and uncomfortable corsets. On the other hand, for generations after that, mainstream underwear brands have infiltrated society's perception of the ideal body image. From the 'teardrop' silhouette bra of the $70 \mathrm{~s}$ to the rounded and lifted bra shape of the $80 \mathrm{~s}$, salient advertisement campaigns have shaped perceptions of normality and desirability, promoting a narrow conceptualization of beauty and femininity. However, in the recent years there has been a movement to increase female body positivity. Many brands feature a celebration of the natural female form, incorporating a diverse range of body types to transcend normative understanding of the 'perfect' shape and size. This thesis strives to address the importance of individuality in bra design to increase a better bra fit for comfort and support.

Women often complain about limitations of size and lack of proper standardization in bra selection. The current common alphabetised "standard" for cup size has partitioned the women's breasts into discrete categories, most commonly, A to F, implying over 3 billion women around the world can fit to these rather arbitrary sizes, when in reality the left and right breast on a single woman may not even be of the same size, or shape. It is essential to understand this gap between women's needs in terms of fit, comfort and aesthetic and what is offered in the market. If cost is not an issue, then each woman could have a idividualised designed and fitted bra, but for a mass consumption of goods (i.e. affordability) to evaluate, use of advanced technology and better design process are needed to manufacture a product that can be used by the masses while still individual.

In earlier projects, an integration of technology with bra design was explored by the author, which later led to the current master's project research. One of the key insights from this research is a glaring lack of female contribution and involvement in the commercial bra design and marketing with specific regard to sizing and fit. Although there has been a recent consciousness of this issue, there is still tremendous room for improvement, to allow greater input by women for a product that is essentially designed for women. The change has been slow but steady, and in the author's opinion, one of the intriguing parts of this type of research. How can women take back the bra so that women are no longer forced to fit into a designated shape, or a shape that has been already designed for them, and instead have a relationship with a bra that molds to the woman just how she desires it to fit? Contemporary bra labels have started to reach out to everyday women, and through avenues such as social media, they have let women demonstrate what a bra means to them, and how they, as individuals, want to wear it and how it should fit and feel. In other words, a level of selfempowerment has been developed through the bra and through inviting women to finally be a part of the story and help guide the process.

Adequate bra comfort and support incorporates a variety of distinct dimensions. Not only does breast size need to be taken into consideration, but also individual breast contour, density and positioning (Chen, LaBat \& Bye, 2010, p. 514). It has been suggested that at least $70 \%$ of women wear the incorrect bra size, with large breasted women accounting for the majority of this discrepancy (Greenbaum et al., 2003, p. 230; McGhee, 2009 , p. 15; McGhee \& Steele, 2010, p. 568; Tsarenko \& Strizhakova, 2015, p. 41; White \& Scurr, 2012, p. 704). The fundamental elements of the current bra fitting system incorporate overbust and underbust size (McGhee, 2009 , p. 144). However, these factors are insufficient for a proper fit. Though some women may have similar band and cup size, any particular bra will not fit these women exactly the same way (Murray \& Michael, 1999, p. 972). Research suggests that the smallest variations in body measurements can cause a significant difference, specifically up to a three size differential in the standard bra sizing system (Liang, 2008, p. 19; White \& Scurr, 2012, p. 704; Wright, 2002, p. 41). Therefore, reliance on the current bra sizing system is left wanting and inadequate for a "perfect" bra fit.

Bra comfort and fit is a strongly subjective domain, and thus adherence to consumer preference is integral in determining correct bra style and fit. However, due to the variance in bra retailers' size labeling (Hardaker \& Fozzard, 1997, p. 314), it has been suggested that women struggle to acquire a well-fitted bra (McGhee \& Steele, 2010 , p. 568). The bra sizing system of many bra labels are inconsistent and indeed arbitrary, leaving women without a reliable method of selection. Even if there was an agreement for a universal standard of bra sizing, millions of women who do not necessarily fit accurately into these units of increment, or more importantly do not have symmetrical sized breasts, would be left neglected and marginalized. Distinct breast shapes, bust distance and distribution of breast muscle, and density collectively must be taken into consideration for bra design and fit (Chan et al., 2001, p. 38). Not only does a well-fitted bra reduce discomfort and pain a good bra fit also carries greater psychological significance (Mills et al., 2014). A correct bra fit can help increase self-confidence and alter negative body perceptions, an integral aspect of psychological functioning that disproportionately impacts women worldwide (Mills et al., 2014). 
This research will attempt to provide practical and useful information for the improvement of bra design through achieving better bra fit. This thesis strives to further the understanding and ultimately help improve the daily struggles of the everyday woman. Chapter 02, Background, provides an extensive review of the current state of research, as well as industry practice and gaps. Chapter 03, Bra System, bases the research within a product-service-system and mass customisation framework and outlines the current, versus the proposed bra system model. Additionally, this chapter establishes the initial design criteria, based on the background knowledge, to which will influence the physical design output. Chapter 04, Methodology, describes the methods used at each stage of the research. Furthermore, Chapter 05, Bra Experience, introduces the participants used in the study. Through semi-structured interviews and questionnaires, the participants help give the author a better understanding of personal bra experiences with design and fit. Using this insight, a range of design themes based on limitations from background research and concerns expressed by the participants. The themes are discussed in Chapter 06, Design Themes, to further drive the design process and final outcome. Chapter 07, Breast Data Collection, explores 3D scanning techniques and a parametric design process as a means to establish necessary measurements through landmarking points. Chapter 08, Bra Design Development: 3D Knitting Exploration, discusses the iterative process of gathering insight into the capabilities of $3 \mathrm{D}$ knitting in the context of bra design. The following chapter, Chapter 09, Bra Design Development: Utilization of Measurements, then uses the knowledge from Chapter 07 and Chapter 08 to compare these non-traditional methods with the traditional bra manufacturing technique of "cut and sew". The final design chapter, Chapter 10, Results, deliberates the results of the preceding chapter and addresses participant feedback on the final bra prototypes. This proposed bra system uses individualised measuring and digital manufacturing methods to design and produce a bra that aims to offer an inherently better fit. 


\section{How might digital technology facilitate a better bra fit beyond cup \& girth size?}

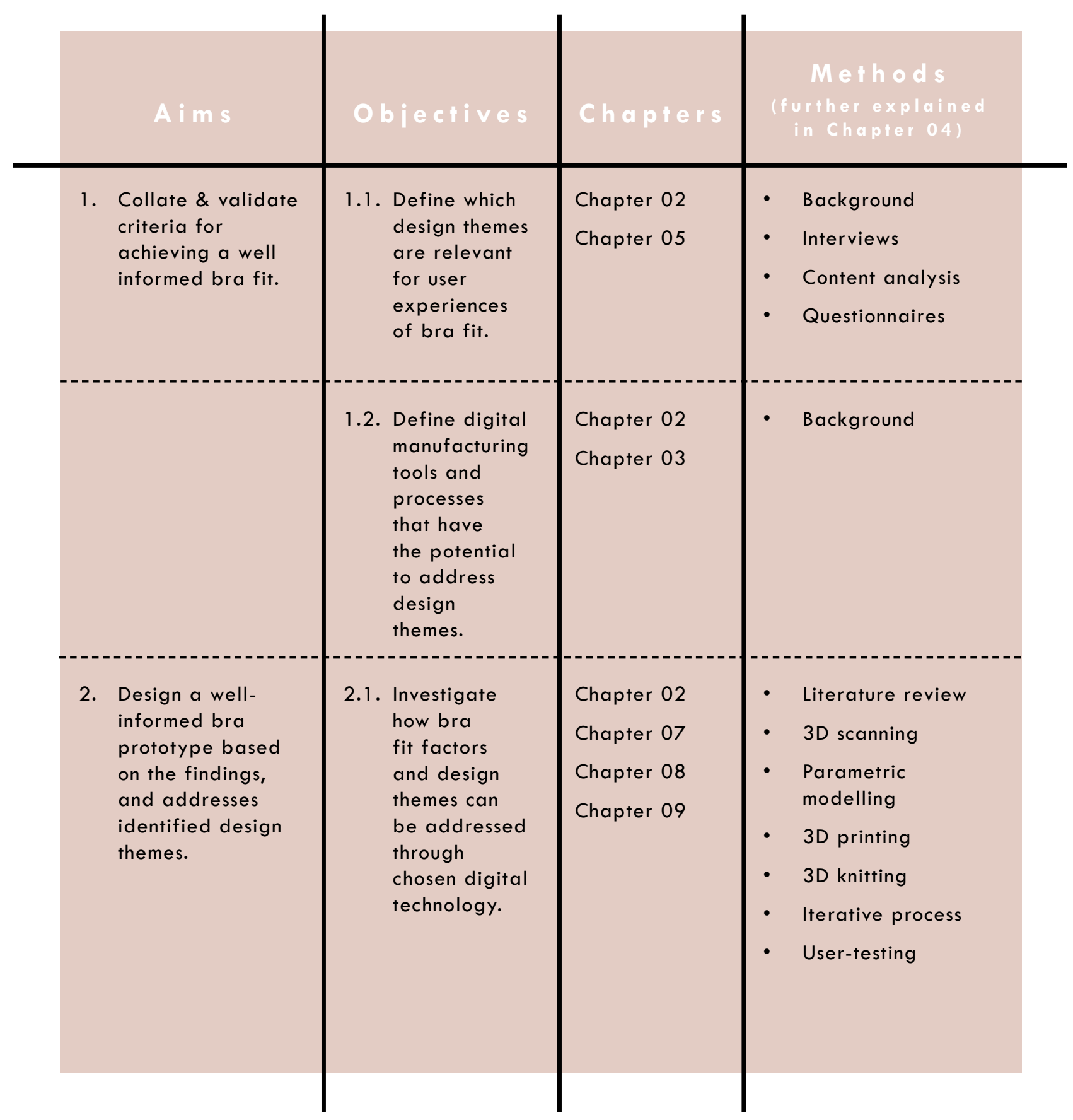




\section{A N A T O M Y O F T H E B R A}

* This is a general description of bra components. Parts may vary depending on bra style.

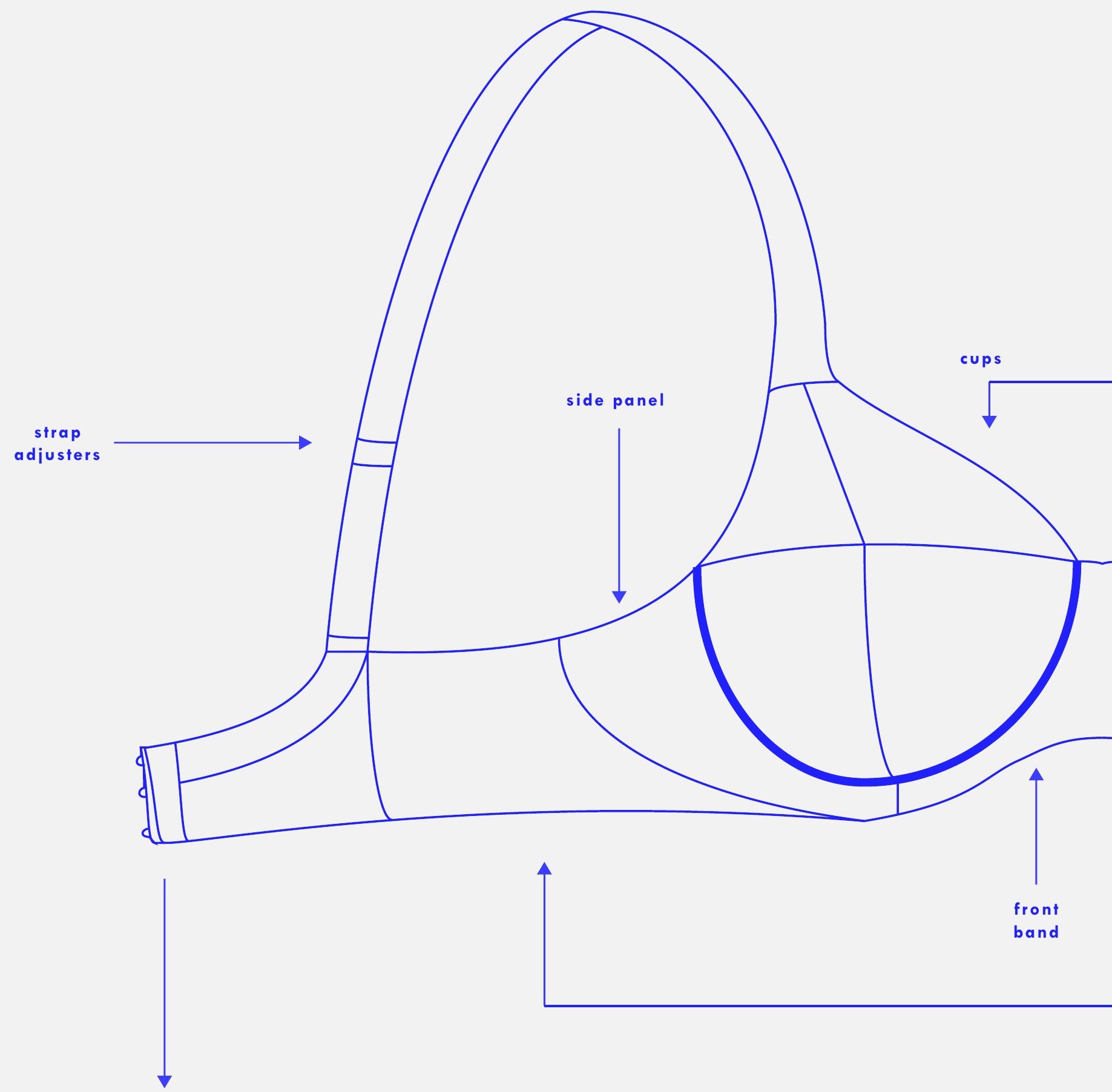




$$
8
$$



C H A P TER 02

B ACK GROUND 
The research area of bra fit and design spans a multitude of disciplines, from medical sciences to sports science, from textiles and fashion to technology and engineering, and from business to market based research. Section I of this chapter will explore women's experiences and attitudes towards the purchasing of bras, as reported through the literature. This section will present an analysis of the recent responses by manufacturers and retailers to changes in traditional purchasing methods. These changes have brought a new paradigm shift in bra buying and the fitting experience through online commerce. Section II will provide an overview of current bra sizing and traditional fitting methods. The literature review will be based on present bra sizing calculations and lack of a standardization between bra sizes, brands and styles. Section II will also outline the impact of incorrect bra fit on the wearer and how differen $t$ fitting methods can provide a better fit. Section III will address existing methods of breast measurements for bra fit and design, including breast topology and volume calculation- a specific aspect of breast size that is difficult to profileand the limitations of current technologies within the scope of this research area. This section will also highlight specific breast measurement parameters that are advantageous to bra fit and design. Section IV will outline the design process of the bra, including traditional and contemporary methods of bra construction, pattern development and bra design innovation. Section V will explore a range of advanced technologies, and how they might be able to aid in breast measuring and bra development. The discussion in this chapter brings together comparable studies in order to justify further specific developments in the area of bra fitting and bra design. 


\section{USER EXPERIENCE}

It is surprising to note many women have many problems with the fit of bras, even though bras have been in use for over a hundred years, and its invention has allowed some sense of comfort and a degree of aesthetic pleasure. Nevertheless, bras continue to be a source of displeasure and discomfort (Yu et al., 2006, p. 140-141). Presently, bras available in the market are limited in sizes and styles, tend to fit improperly, and at times painful to wear to the point that they have become detrimental to one's health. There are of course opportunities for a positive experience. According to one study (Hart \& Dewsnap, 2000, p. 113), consumers have had positive experiences under certain conditions such as when they found a suitable bra and would return to make a similar or repeat purchase; when they had excess money to spend and if the bras were a standard size; or when shopping for a bra to compliment a special outfit or occasion. In other words, comfort, consistency, reliability of function, and variety of specificity of design can provide a positive experience for the consumer. 


\section{Purchasing risks}

One does not think of risk as part of a garment purchase, but no other piece of clothing is so fraught with negative impact on comfort and pleasure. This is not a trivial point as the selected bra can only be assessed properly after it has been purchased, then washed and worn, a decision process that is highly sensitive to the consequences of a "mis-purchase" (Hart \& Dewsnap, 2000, p. 113). Both Hart \& Dewsnap (2000) and Tsarenko \& Strizhakova (2015) discuss bra purchasing as a high-risk activity, due to the difficulties that consumers experience in securing a well-fitted and physically comfortable bra. An ill-fitted bra has been noted to result in discomfort, stress and even lasting bodily injury and lasting body stresses (Greenbaum et al., 2003, p. 230; Oh \& Chun, 2014, p. 301; McGhee \& Steele, 2011, p. 351). A major factor of finding the right fit is due to the lack of consistency, and wide variation in sizes across different brands, even within the same style from the same manufacturer (Hart \& Dewsnap, 2000, p.113-114). Even if a well-fitted bra is found, most consumers feel anxious as they have little confidence in that particular bra style staying on the market and being available for purchase in the future. Moreover, respondents, in Hart \& Dewsnap (2000) study, have reported widespread disillusionment because of past incorrect measurements, which increases the probability of a mispurchase (p. 114).

\section{Buying trends and attitudes}

In contrast to other types of apparel, there is little research on the consumer behavior, attitudes, and trends for this category of intimate apparel. Hart \& Dewsnap (2000) investigated everyday bra buying behaviour and found that more than $60 \%$ of the participants described their bra shopping process as "a nightmare, frustrating... and traumatic" (p. 113). They found that the strongest reason consumers did not enjoy this experience was the difficulty in finding bras that offer good fit, the lack of choice, especially in larger sizes, and the right breast shape (p. 113). The majority of dissatisfaction from the bra buying experience stems from the lack of attractive bras in larger sizes and a lack of choice in general. Tsarenko \& Strizhakova (2015) confirmed the earlier results by showing consumers finding bras that have desirable fit and design have had enhanced shopping experience (p. 42). Furthermore, they show this positive experience in turn can lead to increased consumption. This suggests that companies, at minimum, should try to develop new fitting processes in which consumers, can find satisfaction and become repeat customers.

\section{Bra fitters}

Studies have shown women do not commonly use professional bra fitting services, but rather independently select their bra size with or without trying on any bras (McGhee \& Steele, 2010, p. 568; Tsarenko \& Strizhakova, 2015, p. 45). McGhee \& Steele (2010) suggest that this is because bra fitters are not readily available at all locations, where bras are purchased (p. 568). Unlike the social interactions associated with other fashion apparel shopping experiences, research shows the vast majority of women prefer to shop alone when purchasing intimate apparel (Hart \& Dewsnap, 2000, p. 114). Tsarenko \& Strizhakova (2015) found that aversion to use of bra fitting services is especially prevalent amongst younger women, as they tend to rely more on the advice and assurance from peer group (p. 45). Additionally, it is noted that women with larger breasts may experience higher levels of self-consciousness during bra fitting, and thereby attempt to size and fit the bras independently (Wood, 2009, p.4).

Even when bra fitters are available, the high variability between experience, technique and accuracy leaves consumers with additional risk of error and may results in an ill-fitting bra purchase (Greenbaum et al., 2003, p. 234). McGhee \& Steele (2010) suggest new research should focus on improving the ability of women to independently choose a correctly fitted bra (p. 571). In other words, if the necessary information can be provided to the consumer regarding how to achieve a good bra fit, then this could help the consumer's ability for selfselecting better bra fit and enhance the satisfaction they receive from all bras.

\section{Online fitting}

Many bra retailers have attempted to redefine the bra buying and fitting experience by offering them either exclusively online or as a combination of online and traditional ("brick and mortar") store. These retailers are trying to address the complaints and negative experiences women have confronted in traditional stores. For example, retailers are trying to respond to the risk of mis-purchase and disappointment with bra fitting in stores, by using surveys and questionnaires. This is an attempt by retailers to find out details about body type that are crucial to a correct bra fit, which as stated above, are frequently are not shared with a salesperson in traditional shops. Below, is a sample of some of the retailers that are attempting to collect data to assist consumers with their bra selection. 
True \& Co.
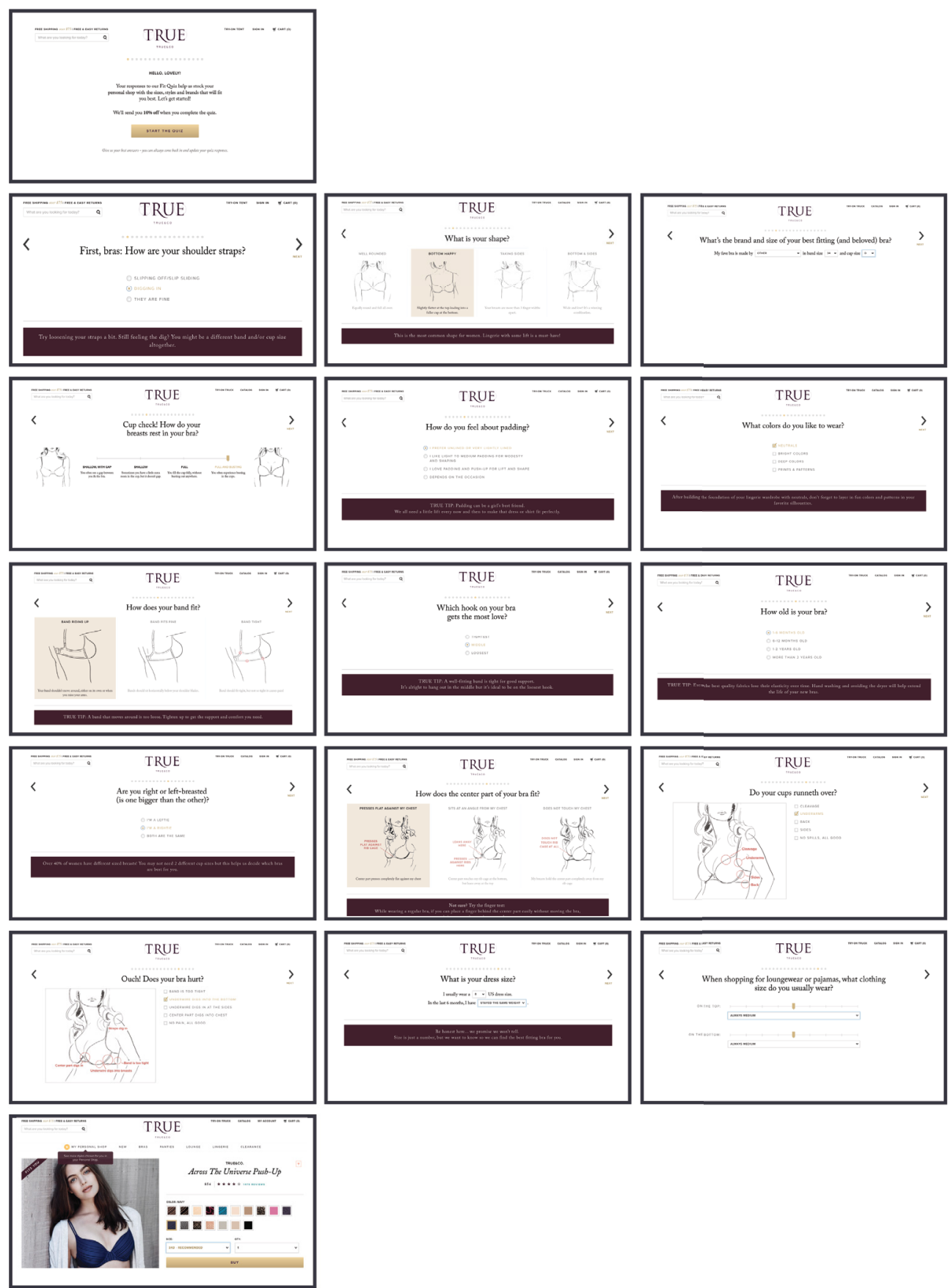

Proper fit is so crucial that over 20,000 of women signed up within 36 hours after True \& Co., a San Francisco startup retailer for intimate apparel, opened its web site in May 2012 (Olson, 2012). The True \& Co. method is an inception of two former Microsoft employees, who devised a computerized method to connect customers to a correct bra fit (Olson, 2012). True \& Co., started their online retail store based around survey responses from thousands of women and separating out the data to create profiles of size and body types (McCall, 2014). The True \& Co. method begins by using a questionnaire to determine the user's bra size. The questions revolve around the fit of one's current bra, including problematic straps and cups. It asked about body shape and clothing size. True \& Co. uses this data to assign the customer a size and a body type. 

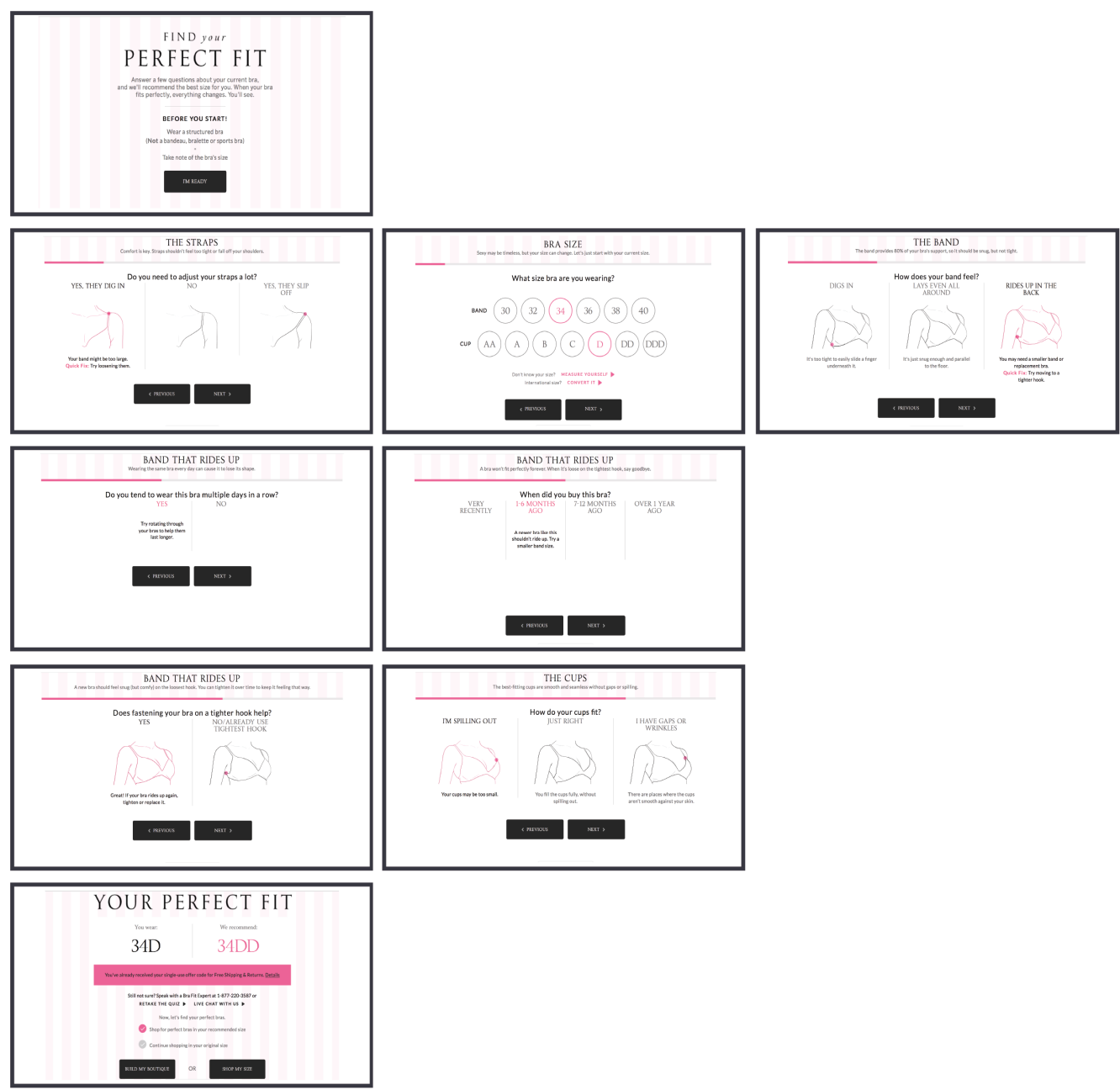

Similar to True \& Co., Victoria Secret's Fit Quiz, established in 2012, uses a survey format to reveal the user's correct fit. Depending on th answer given, there are up to nine different types of questions in the quiz. These questions ask the user to observe the bra they are currently wearing and state any concerns or fitting problems they encounter. Some questions even entail asking how long the bra has been worn in a given year. 

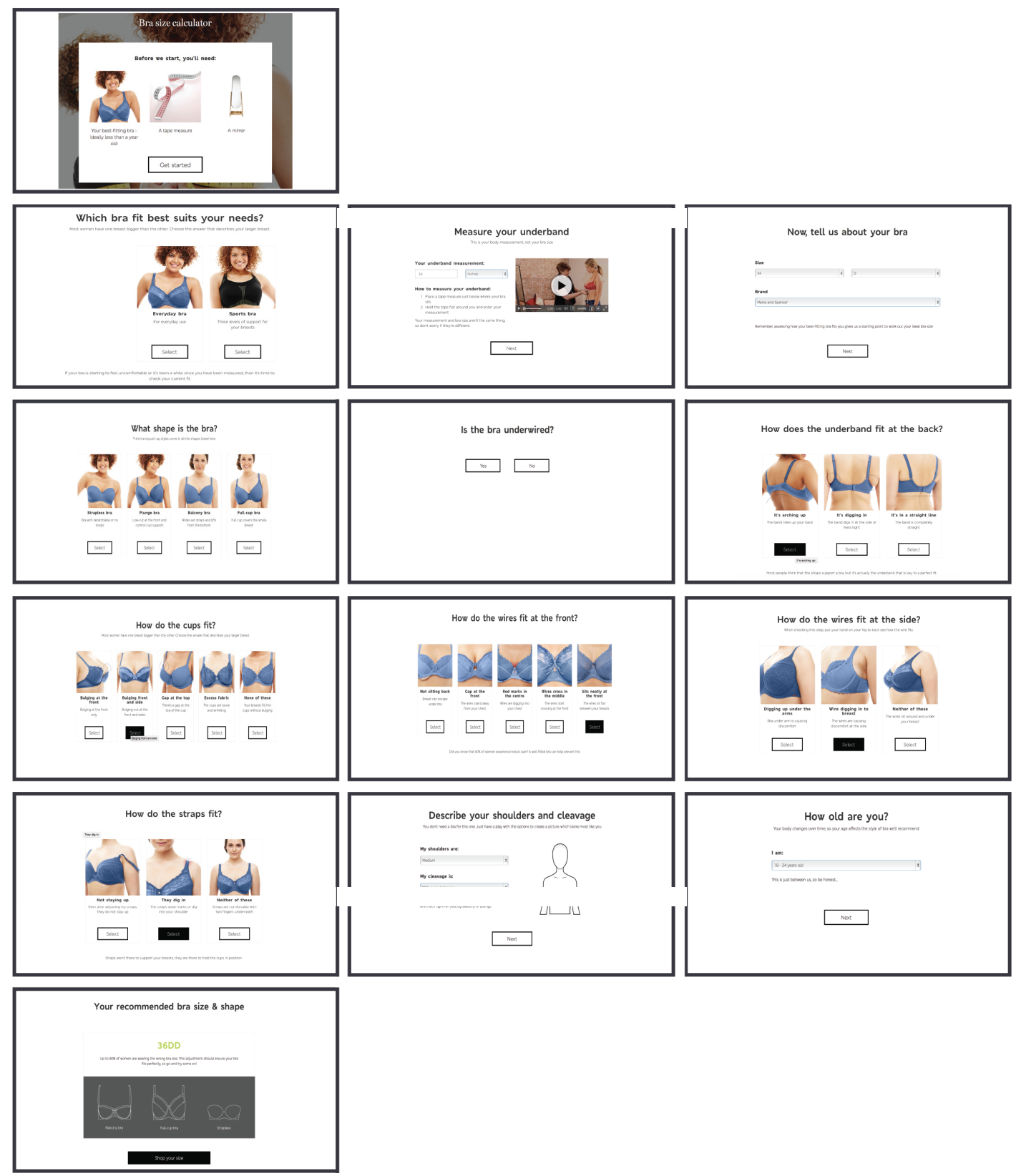

Marks and Spencer, a British retailer, also has a "Fit Quiz" to help users calculate their correct fit. Besides the usual questions about cup, band and strap fit, Marks and Spencer's questions also include bra style, age, and body shape. There are also detailed questions about the underwire and how it sits against the body, which has not been a question in any other fit quizzes. 

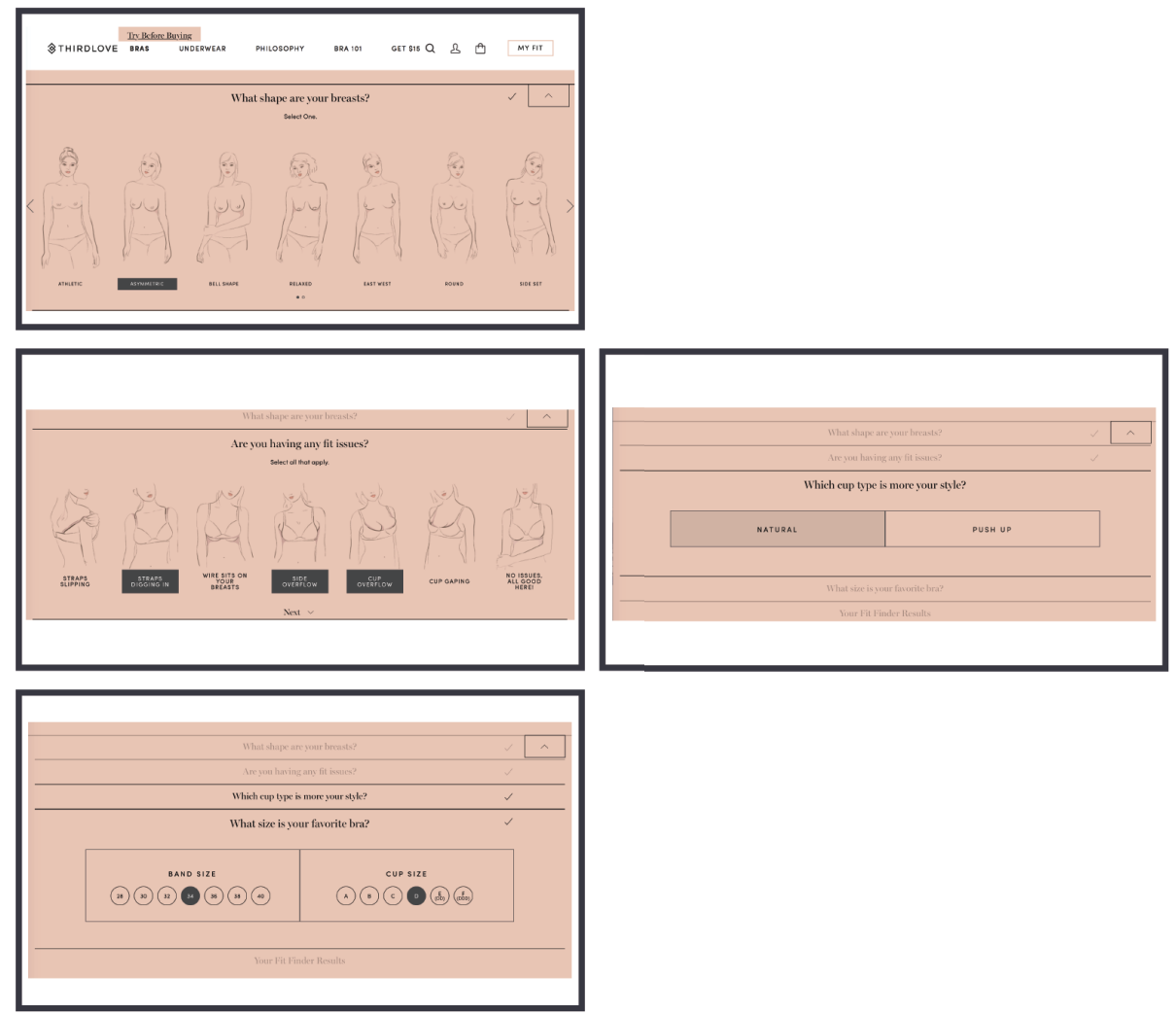

ThirdLove is an example of a successful start-up attempting to disrupt traditional approaches to buying and fitting bras, using commonly used technology. ThirdLove developed an app for smartphones that combines both the fitting process and the shopping experience. The process involves taking two photos, one front facing and one side facing, in a mirror. The app suggests wearing a non-padded underwire bra or a tight tank top. The algorithm developed by former Google employees, calculates the size of the user's chest and body in relationship to the phone itself. Besides the app, they also have a bra fitting quiz that can be completed online. This quiz is however, less detailed, in that there are minimal questions about the fit of the bra, and less measurements needed from the user. 


\section{Online quiz analysis}

These quizzes are attempting to reinvent the bra buying experience through a more private and convenient manner. This process has allowed women to become their own bra fitters and help narrow down the search for the correct fit. However, the majority of these online quizzes have inadvertently limited the options for users, as the results direct the women to a few bra selections. True \& Co. and ThirdLove recommend the user's bra size and then narrow the selection of bra choices and styles, based on designated size, followed by additional recommendations for purchase from their in-house collection. Victoria's Secret, on the other hand, only confirms a bra size but does not limit the selection of bras available to purchase based on the results. ThirdLove has taken this one step further by incorporating a voice on the phone which advises the user if they are doing something that will render the results useless. ThirdLove also responds to limitations in bra sizes by introducing half-cup sized options, which is a novel attempt at more precise measurements of the breast.

There are however, overwhelming limitations that outweigh the successes of using quizzes and surveys as a tool for better fit. Firstly, the use of online quizzes and surveys as a tool for better fit is questionable because of the uncertain reliability of customers self-reporting. Overall, there is too little, if any, bra fitting education and training and therefore it is difficult for women to know what they should be looking for when reporting their bra fit. Also quizzes like ThirdLove, which measurements stem from the bra that the consumer is currently wearing, are inherently prone to errors when they serve the basis for new size results. ThirdLove's algorithm requires wearing a bra (presumably a correctly fitted bra), and thus it is probable the results of a user with an ill-fitting are likely to have errors.

\section{"This [online fitting] process has allowed} women to become their own bra fitters and help narrow down the search for the correct fit." 
SECTION II.

SIZE \& FIT

The current state of bra fitting relies on only two factors: overbust and underbust size (McGhee, 2009, p. 144), and bras come with incomplete information reflecting these two metrics. For example, a bra may be labeled as $12 \mathrm{C}$ in New Zealand (or say, 34C in the U.S.), which presumably reflects the underbust and overbust respectively, but not all the $12 \mathrm{~s}$ are of the same length and not all the Cs are the same cup volume. Therefore, it is not surprising that recent research has shown at least $70 \%$ of women are wearing the wrong sized bra, with the fitting discrepancy greatest in larger breasted women (Greenbaum et al., 2003, p. 230; McGhee, 2009, p. 15; McGhee \& Steele, 2010, p. 568; Tsarenko \& Strizhakova, 2015, p. 41; White \& Scurr, 2012, p. 704). Even if bra manufacturers followed the same standard, a particular bra will not fit two women exactly the same way, even though they may have the same bust girth and cup size (Murray \& Michael, 1999, p. 972). This is because these two metrics are not sufficient to assess fit of a bra, as breasts do not come in standard sizes and shapes. A perfectly fitted bra is important for not just comfort and aesthetics, but also for health. Therefore other factors such as bust distance or distribution of breast muscle or density must be part of the calculus (Chan et al., 2001, p. 38). 


\section{Bra Sizing}

In 1926, less than twelve years after Mary Phelps Jacob received a patent for the first bra design that is commonly recognized as the basis for modern bras, there were attempts to establish a bra sizing system (Morris et al., 2002), where breast shapes were classified into analogous types. Later, Berlei Underwear Company in Australia carried out a size survey to study women's figures between 1926 and 1928. It primarily used the circumference of the women's chest, bust and underbust to characterize the bra size. Subsequently in 1935, Warner, an American company, incorporated the volume of breasts into the bra size specification (White \& Scurr, 2011, p. 704). It first advertised the alphabet of bras as "A" cup for youthful, "B" cup as average, "C" cup as large and "D" cup for heavy (Zheng et al., 2007, p. 697). Consequently, this system became the foundation of modern bra sizing. Overall, two major bra sizing systems have been generally used since the introduction of Warner's alphabetic bra sizes: imperial and metric systems (White \& Scurr, 2012, p. 704), though other variability exists as well. Figure 2.5. illustatres some of this variety of sizing and labelling across the world.

However, since its adoption, body sizes and shapes have changed considerably, with the average cup size rising from a B cup to a D cup or larger. This shift has not yet been acknowledged by the majority of bra companies and thus, current sizing system does not reliably reflect women's actual shape and contour. Furthermore, it needs to be argued that even if body sizes and shapes had stayed static, a simple lettering scheme does not sufficiently address the multitude of sizes and shapes across the world. In particular, studies suggest that bra cup size does not accurately indicate actual breast volume (Oh \& Chun, 2014, p. 301), and as will be discussed further in Section III. Breast measurement, breast volume calculation methods vary as well. For example, the current system does not take into account the natural occurrence of asymmetry within any given pair of breasts (further discussion in Section III).

\section{Inconsistencies in sizing, brand, \& style}

Due to inconsistencies across bra sizes and brands, a majority of women struggle to find correctly sized bras, and empirical evidence shows bra sizing as more of a trial and error process than anything else. A 1997 study of professional bra design practice compared multiple bra design manufacturing companies to assess the design process. In this study, it was observed that while all manufacturers used the same size notation, there was no industry-wide size chart, and therefore each manufacturer used its own company's chart to size the bras (Hardaker \& Fozzard, 1997, p. 314). Consequently, there are large inconsistencies across bra labeling, and the true sizes of bras are unknown despite their associated labels, if they are produced by different manufactures. This lack of a standardized set of measurements has led to a nonuniform size chart, thereby making bra consumers quite confused. Even within the same notation of alphabetized cup sizes, further confusion exists, as the actual size of the bra cup varies with the band size (Liang, 2008, p. 19).

McGhee \& Steele (2010) compared breast volume to corresponding correctly fitted bra size to demonstrate the range of volumes within each bra size. This study also showed inconsistencies between cup sizes across various brands. For example, a 10D labeled bra (D cup size) does not have the same cup size as $12 \mathrm{D}$. This study recommended, what many women know by experience, that letters associated with cup size cannot be considered in isolation and they should consider the brand when judging the cup size.

As argued earlier, even if there were a reliable international or domestic standard for bra sizing, it still would leave millions of women who don't necessarily fit well into current units of increment and perhaps even more importantly have dissimilar sized and shaped left and right breasts (i.e., asymmetry). Relying on the current standard calculated bra sizing system is not enough for a proper bra fit. Miscommunication of bra sizes is one of the main reasons that leads to inaccuracies in bra fit (Chen et al., 2011, p. 695). That is, very few women wear bras that fit them properly and this has resulted in discomfort, stress and even lasting bodily injury and lasting body stresses (Greenbaum et al., 2003, p. 230; Oh \& Chun, 2014, p. 301; McGhee \& Steele, 2011, p. 351). 


\section{N T E R N A T I O N A L B RA S I Z E CON VERSION}

\begin{tabular}{|c|c|c|c|c|}
\hline $\begin{array}{c}\text { New } \\
\text { Zealand } \& \\
\text { A ustralio }\end{array}$ & USA & $\begin{array}{l}\text { U K } 8 \\
\ln \text { di a }\end{array}$ & $\begin{array}{l}\text { Europe, } \\
\text { Chine, } \\
\text { Jopon, } \\
\text { Hong Kong } \\
\text { \& Kored }\end{array}$ & $\begin{array}{l}\text { Spain, } \\
\text { France \& } \\
\text { Belgivm }\end{array}$ \\
\hline $10 \mathrm{~A}$ & $32 \mathrm{~A}$ & $32 \mathrm{~A}$ & $70 \mathrm{~B}$ & $85 B$ \\
\hline $10 \mathrm{~B}$ & $32 \mathrm{~B}$ & 32 B & $70 \mathrm{C}$ & $85 \mathrm{C}$ \\
\hline $10 \mathrm{C}$ & $32 \mathrm{C}$ & $32 \mathrm{C}$ & $70 \mathrm{D}$ & $85 \mathrm{D}$ \\
\hline $10 \mathrm{D}$ & $32 \mathrm{D}$ & $32 \mathrm{D}$ & $70 \mathrm{E}$ & $85 \mathrm{E}$ \\
\hline $10 \mathrm{DD}$ & $32 \mathrm{DD}$ & $32 \mathrm{DD}$ & $70 \mathrm{~F}$ & $85 \mathrm{~F}$ \\
\hline $10 \mathrm{E}$ & 32 DDD & $32 \mathrm{E}$ & $70 \mathrm{G}$ & $85 \mathrm{G}$ \\
\hline $10 \mathrm{~F}$ & $32 \mathrm{~F}$ & $32 \mathrm{~F}$ & $70 \mathrm{H}$ & $85 \mathrm{H}$ \\
\hline $12 \mathrm{~A}$ & $34 \mathrm{~A}$ & $34 \mathrm{~A}$ & 75 B & $90 \mathrm{~B}$ \\
\hline 12 B & $34 \mathrm{~B}$ & $34 \mathrm{~B}$ & $75 \mathrm{C}$ & $90 \mathrm{C}$ \\
\hline $12 C$ & $34 \mathrm{C}$ & $34 \mathrm{C}$ & $75 \mathrm{D}$ & $90 \mathrm{D}$ \\
\hline $12 \mathrm{D}$ & $34 \mathrm{D}$ & $34 \mathrm{D}$ & $75 \mathrm{E}$ & $90 \mathrm{E}$ \\
\hline $12 \mathrm{DD}$ & $34 \mathrm{DD}$ & $34 \mathrm{DD}$ & $75 \mathrm{~F}$ & $90 \mathrm{~F}$ \\
\hline $12 \mathrm{E}$ & 34 DDD & $34 \mathrm{E}$ & $75 \mathrm{G}$ & $90 \mathrm{G}$ \\
\hline $12 \mathrm{~F}$ & $34 \mathrm{~F}$ & $34 \mathrm{~F}$ & $75 \mathrm{H}$ & $90 \mathrm{H}$ \\
\hline $14 \mathrm{~A}$ & $36 \mathrm{~A}$ & $36 \mathrm{~A}$ & $80 \mathrm{~B}$ & $95 \mathrm{~B}$ \\
\hline $14 \mathrm{~B}$ & $36 \mathrm{~B}$ & $36 \mathrm{~B}$ & $80 \mathrm{C}$ & $95 \mathrm{C}$ \\
\hline $14 C$ & $36 C$ & $36 c$ & $80 \mathrm{D}$ & $95 \mathrm{D}$ \\
\hline $14 \mathrm{D}$ & $36 \mathrm{D}$ & $36 \mathrm{D}$ & $80 \mathrm{E}$ & $95 \mathrm{E}$ \\
\hline $14 \mathrm{DD}$ & $36 \mathrm{DD}$ & $36 \mathrm{DD}$ & $80 \mathrm{~F}$ & $95 \mathrm{~F}$ \\
\hline $14 \mathrm{E}$ & $36 \mathrm{DDD}$ & $36 \mathrm{E}$ & $80 \mathrm{G}$ & $95 \mathrm{G}$ \\
\hline $14 \mathrm{~F}$ & $36 \mathrm{~F}$ & $36 \mathrm{~F}$ & $80 \mathrm{H}$ & $95 \mathrm{H}$ \\
\hline
\end{tabular}




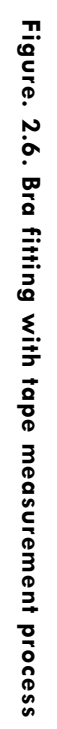
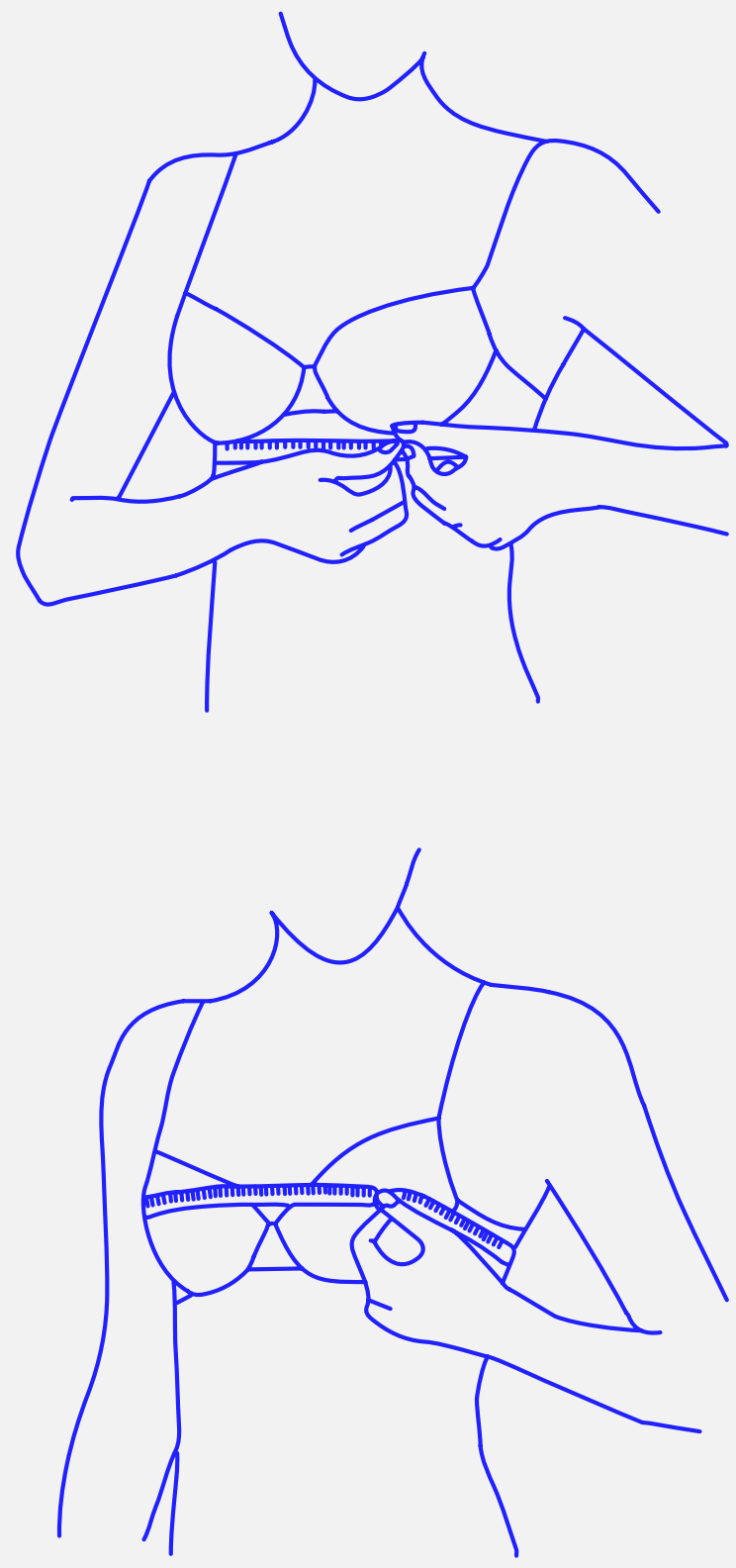

$\begin{array}{lllllllllllllllllllll}\text { T R A D I T I O } & \text { N A L } & \text { B R A } & \text { F I T } & \text { T I } & \text { N G }\end{array}$

$\omega$ 


\section{Flaw of averages}

Bra sizing is determined through the development and design of an initial sample bra, "base bra", and the majority of bra companies use an average bra size for this process (Hardaker \& Fozzard, 1997, p. 312). This sample bra is then used for a selection of sizes. Though every bra brand and company may have its own set of sizing standards, they all use this grading system based on an average to create a range of sizes.

In his book, "The End of Average", Rose (2016) discusses the flaw of designing for an average. He explains that the use of average could be useful in cases of comparing two different groups of people, but when it comes to making a decision about the individual (i.e, identifying individual traits), then the use of average becomes useless. Rose goes on further to say that using an average to design for an individual "creates the illusion of knowledge" (p.11). In a sense, the over reliance on the use of average covers up what is unique to that individual. The study questioned whether anyone actually has the sizes of the average sample. Their results, not surprisingly, show no single person fits into the average measurements. In turn, designing for an average means designing for no one.

This poses a question to whether one of the solutions to the problem of bra fit can be solved by reviewing and reassessing the current bra grading methods and building bra sizes based on a sample size of an average. Standardization seems neither possible nor helpful for bra designing, and seems to only help outdated manufacturing processes. Bra size notation may also be unnecessary. Since all sizes are dependent not only on the bra brand but also the bra style, size notation may be more problematic than useful.

\section{Traditional bra fitting method}

The traditional method of bra fitting, using the imperial system, is based on measuring the chest circumference (underbust circumference) and the circumference of the chest around the fullest part of the breasts (overbust circumference), as displayed in figure 2.6., (White \& Scurr, 2012, p. 704). To determine band size, an arbitrary number (commonly 4 or 5 ) is added to the underbust chest circumference in inches (White \& Scurr, 2012, p. 704). The cup size, which is presumed as a description of breast volume, is then established by calculating the difference between the band size measurement and over the bust measurement (White \& Scurr, 2012, p. 704; Zheng et al., 2007, p. 697). There has been much criticism of achieving cup size through this method, as it has been repeatedly proven as inaccurate (McGhee \& Steele, 2010, p. 570; Pandarum et al., 2011, p. 866; Zheng et al., 2007, p. 32-33). Small errors due to a rounding process in the initial band and cup measurements may lead to an error of up to three cup sizes (Liang, 2008, p. 19; White \& Scurr, 2012, p. 704; Wright, 2002, p. 41). Furthermore, the traditional bra fitting method, commonly used by retailers, was designed for cup sizes up to a " $D$ " cup volume only, and thus the accuracy of this method with sizes larger than a D cup is uncertain (White \& Scurr, 2012, p. 705). Additional bra fit criteria must also address the structural differences between the fit needs of smaller breasts, compared to larger ones. Marks and Spencer is one of the few retailers that provide bra fit criteria between A through D cup with additional specific criteria for cup size DD plus. Thus, it is no surprise that the majority of the women who face the most difficulty in fit and sizing are larger breasted women. Consequently, women with larger breasts are more likely to be wearing incorrectly sized and ill fitted bras (Wood, 2009). For example, when taking the underband measurement to fit a bra, there is a risk to cut into the excess flesh with the tape measure which in turn maximizes the inaccuracy of the cup-size measurement (Greenbaum et al., 2003, p. 235; Wood, 2009).

White \& Scurr (2012) have discussed inaccuracies in this method and suggest the need for women of all breast sizes to be wary of using traditional bra fitting techniques to assess their bra fit (p. 710). They recommend the bra size should be measured over a correctly fitted bra in order for the new bra to be fitted correctly, and note that retailers do not always follow this best practice. If the fit of a bra that is worn when taking measurements impacts the size calculation of the fit of the new bra, then there exists a weakness in the traditional fitting system. Unfortunately this same method of measuring over one's own bra continues to be used as was illustrated in a recent online fitting guide, offered by ThirdLove, which lead to unreliable results and ill-fitting bras, as explored in Section I. 


\section{Bra fit criteria}

Bra fitting is traditionally a subjective judging process, which relies on the experience of a bra fitter for assessment (Fong, 2014, p. 27). After a person has been measured (e.g.overbust and underbuist measurements) and a bra size has been determined, current bra-fitting principles recommend double-checking the fit while worn. This step would help the selection based on how the sidebands, cups, underwire, straps and front band fit against the body (McGhee, 2009, p. 32). A bra fit criteria can be used as a checklist for at least achieving adequate bra fit (Mitchell, 2013, p. 1-2). Figure 2.7. demostrates a collated list of bra fit criteria based on idustry and academia standards.

In 2005, Choice Magazine developed a set of criteria for bra fit that has been utilized and validated by McGhee $\&$ Steele (2010) with a reliability of $92 \%$ for those criteria (p. 569). However, Choice magazine's criteria (like most industry criteria) do not cover all aspects of bra fit suggested in the literature as essential, this points to the possibility that the industry and academic criteria are developed independently of each other and are not meaningfully coordinated. Additionally, it is noted that a bra, which could fulfill all the bra fit criteria might not always represent a good fit for the individual (Fong, 2014, p. 28). That is, individual preference must be part of any equation. A well-fitted bra should also be able to look and feel good. Most would agree the aesthetic aspect is highly subjective but comfort also depends, at least partially, on the individual. The subjective portion of the look and feel cannot simply be measured by a set of criteria independent of the person. For example, comfort and support may be defined differently depending on the wearer. This makes bra fit process highly complex and difficult to measure.

\section{Bra fitting}

McGhee \& Steele (2010) were the first to observe consumer's bra selection process and assess the results against professional fit criteria. In particular, they considered three methods consumers have used for finding the correct bra size: (1) trial and error, (2) chest circumference method (also referred to as underbust measure) and (3) the breast hemi-circumference method (also referred as overbust method) (p. 570). Their research shows a positive correlation between trial and error method and the bras participants actually wear. Their work suggests participants could independently select the same fit (i.e., consistency) but lacked the knowledge to ascertain what is determined as a good fit (p. 571). The findings of the study suggest that an increase in knowledge could improve bra fit but does not identify any specific gaps in the bra sizing systems used.
In addition to uneven bra fitter training, variability in methods and techniques leads to unreliable bra fitting results. Greenbaum et al. (2003) looked at the different ways bra fitters measure the over-bust circumference (explained above as part of the traditional fitting method). The variability included measuring over the participant's own bra, or asking the participant to lift the nude breasts to desired shape and then measuring, and manually measuring the nude breast. Due to general trepidation by most bra fitters to physical touch nude breasts, the most popular form of over-bust measurement is over the bra. However, once again the measurements are not meaningful if the consumer's own bra does not fit appropriately (Greenbaum et al. 2003, p. 234). A measuring system would thus need to account for the unreliability of the resulting measurements due to bra being worn, or develop a measuring technique that avoids a unfit bra while being measured. 


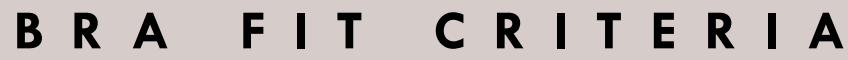

Collated list of bra fit criteria based on industry \& academia standards. A full list of bra fit criteria can be viewed in Appendix I.

\begin{tabular}{|c|c|}
\hline 01 & Primary support should come from the band \\
\hline 02 & Does not ride up \\
\hline 03 & Fitted on the loosest hook \\
\hline 04 & Follows a horizontal line across the body \\
\hline 05 & At most two fingers should be able to fit underneath \\
\hline 06 & No flesh should bulge out \\
\hline 07 & Fits firm \\
\hline 08 & Allows for comfortable breathing \\
\hline & Cups \& Underwires \\
\hline
\end{tabular}

02 Breast does not bulge or spill over the cups

03 Cup has no wrinkles, gaps or spaces

04 Space on just the top of the cup suggests style flaw

05 Breast should be fully enclosed within the cup / smooth line

06 The underwire should not dig into the skin

07

Wire should not slip down

Strops

Do not dig into the skin / do not leave a red mark

Should sit firm, at most two fingers should be able to fit underneath

Should not slip off

4 Smooth line against the skin

Front band 


\section{BREAST MEASUREMENT}

In order to achieve precise, functional and comfortable fit, accurate and detailed measurements of the breasts are necessary. However, this is not an easy process as such measurements are very sensitive to factors such as breathing and posture (Chen et al., 2010; Pandarum et al., 2011; White \& Scurr, 2011; Zheng et al. 2007). Therefore, in order to collect accurate data, a variety of different measurements of the breasts and upper torso are vital. Currently there are two possible options for breast measurement procedures in the bra fit system. The first is measuring breasts in the manufacturing stages, as part of bra design, patternmaking, and grading development. The second is to measure the breasts in the retail setting when purchasing a bra, used for fitting into the correct bra size of the individual wearer.

It is critical for both bra manufacturers and bra wearers to have an understanding of the significance of breast characteristics (volume, outline, and shape) in relation to bra size. If the goal is to design a better fitting bra, there must be recognition and a better understanding of the structure, support and function bras must provide. Ultimately, the design of bras cannot be independent of the characteristics of the woman's body and breasts. In the remainder of this section, we will discuss the physiology of breasts and all the associated characteristics needed for better measurement. 


\section{T H I R D L O V E \\ B R E A S T S H A PE DI RE C T O R Y}

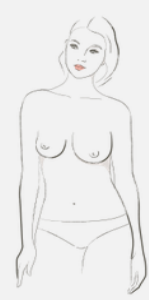

Asymmetric

One breast larger than the other

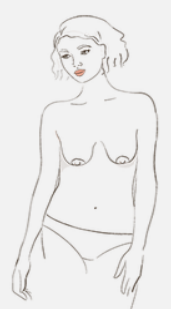

Relaxed

Breasts with lax tissue and nipples pointing downward

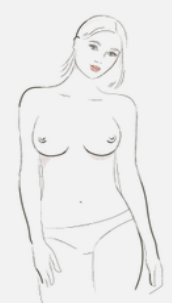

Side Set

Wide space between

breasts

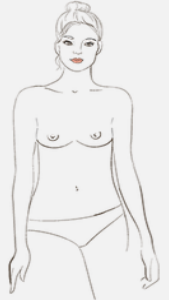

Athletic

Wider more muscular breasts with less tissue

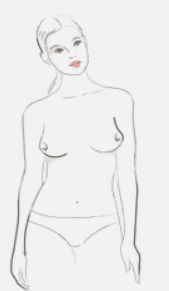

East West

Nipples pointing outward in opposite directions

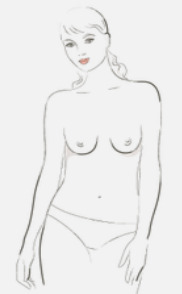

Slender

Thin breasts, nipples pointing downward

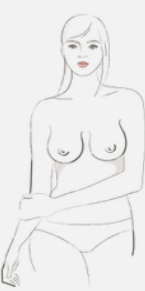

Bell Shape

Slimmer at the top, fuller at the bottom

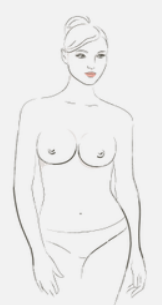

Round

Equally full at the top and bottom

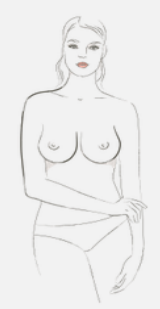

Tear Drop

Round but slightly less full at the top

\section{Breast physiology}

Human breasts are situated on the chest wall between the 2 nd and 6 th intercostal cartilage in the vertical plane (Zheng, 2009, p. 11). There are three major structural components within the breasts: the skin, the subcutaneous tissue and the corpus mammae (Jenkins, 2005, p. 112). However, since the breasts have no muscle tissue to provide support, the ligaments and the fibrous tissues become the internal anatomical support (Gehlsen \& Stoner, 1987). Breast shape variations have been categorized in medical fields as "hemispherical, conical, variably pendulous, piriform, thin and flattened" (Standring \& Gray, 2008). The limitation with these definitions is the underlying assumption of uniformity and symmetry, neither being true of natural breasts. McGhee \& Steele (2011) make reference to categorizing breast shape as "pert", "broad" and "ptotic" (p. 354). ThirdLove uses breast shape as part of their fitting guide, these include six different breast descriptions as demostrated above (figure 2.8.). Though these terms are applicable and sufficient for a broad description, neither set provides traceable reference, and therefore are difficult to validate for this research. Nevertheless, there is understanding that differences in breast shape do exist and will readily impact on the design and fit requirements of a bra. 


\section{Breast volume}

It has been suggested the correct bra fit is where the breast is fully encased within the cup. This implies the volume of the bra cup (not including the outer material) should equate to the volume of the breast within it (McGhee \& Steele, 2011, p. 352), though this may not consider the pressure applied by the bra on the breast tissue. Lee et al. (2004) further explain, it is the "inaccurate measurement of breast volume that causes the most common discomfort and bad bra fit" (p. 353). Therefore, we can hypothesise that accurate measurement of breast volume is a key indicator for designing cups for bras. As mentioned earlier, Warner was the first to develop a system to apply breast volume measurements into corresponding alphabetized bra cup sizes. This system formed the basis for how modern bra sizing is set today. There are several different types of breast volume measurement methods, both manual and technology based throughout several different fields. However, there is no agreed standard on measurement methods or measuring calculation techniques. In fact, and not surprisingly, each method of estimation offers different results of breast volume (Kovacs et al., 2007, p. 144).

The current breast volume measurement methods can be categorized into four groups (McGhee \& Steele, 2011; Bulstrode et al., 2001; Yip \& Yu, 2006):

- Computer-analyzed indirect visualization techniques

- Anthropometric measurements

- Water-displacement techniques", also known as the Archimedes principle

- Thermoplastic moulding

This section will briefly mention current breast volume protocols, giving greater detail to those that can be applied specifically for better bra design and fit.

\section{Computer-analyzed visualisation techniques}

There are three different methods in this technique that are most commonly used for breast volume measurement:

$013 \mathrm{D}$ digital imaging and scanning (Bulstrode et al., 2001; McGhee \& Steele, 2011; Mitchell, 2013; Lee et al., 2004; Kovacs et al., 2007; Yip \& Yu, 2006). Chen et al. (2011) suggests that 3D scanning techniques could advance bra design and revenue by applying data from 3D imaging to develop shapes of bra cups better suited to larger breasts (p. 701). Other researchers seem to concur and have argued that the $3 \mathrm{D}$ scanner has the most potential for breast volume because of its capability for measurement precision and characteristic relationships to the other recognized methods (Kovacs et al., 2007; Lee et al., 2004; Lee \& Hong, 2006). However, the large variation in $3 \mathrm{D}$ surface systems and software applications, which differ in cost and quality, make it difficult to universally validate this method (Simmons \& Istook, 2003; Kovacs et al., 2007). Nevertheless, there have been various ways discussed in literature to utilize the 3D scanner for obtaining breast volume. Chen et al. (2010) suggests the most efficient manner to predict breast volume through a $3 \mathrm{D}$ scanner is to use the bust angles of the 3D model with software such as ScanWorX (p. 517). Chen \& Wang (2015) explored a novel breast volume measuring method by mesh projection based on the $3 \mathrm{D}$ point cloud data gathered from 3D scanning. Lee et al. (2004) proposed slicing and filling the breasts of the $3 \mathrm{D}$ scanned models for effective breast volume (p. 356).

02 Magnetic resonance imaging techniques (Bulstrode et al., 2001; McGhee \& Steele, 2011; Kovacs et al., 2007; Yip \& Yu, 2006). MRI is one of the most precise volume assessments but it is also the most costly (Bulstrode et al., 2001; Kovacs et al., 2007), which makes it rather impractical for everyday bra shopper. MRI scan also has another major disadvantage in that the deformation of the chest region when in the supine position (lying down). The images taken will render the resulting 3D models of the MRI scans with little correspondence to the actual anatomical shape of the breasts (Kovacs et al., 2007, p. 143). 


\section{3} Mammograms (Bulstrode et al., 2001; McGhee \& Steele, 2011; Yu et al., 2006). Mammogram is an $\mathrm{x}$-ray imaging technique of the breasts. They have also been offered as one of the most effective form of breast volume measurement (Kayar et al., 2011; Bulstrode et al., 2001). Calculating the volume from mammography could be rather straightforward, however, not surprisingly this method is the least tolerated method of measurement amongst participants (Bulstrode et al., 2001). However, both Kayar et al. (2011) and Bulstrode et al. (2001) do not consider $3 \mathrm{D}$ scanning in their study, which has also been equivalently validated and is much more accessible (McGhee \& Steele, 2011; Lee et al., 2004; Lee \& Hong, 2006; Chen \& Wang, 2015; Kovacs et al., 2007).

\section{Anthropometric measurement}

Anthropometric measurement (also known as anatomical measurements) is a technique used to quantitatively express the form of the human body. The breast volumes identified through anthropometric measurements are computed based only on individually measured values, and a predefined geometrical shape is imposed on the breast form which does not necessarily correspond to the actual individuals anatomical conditions (Kovacs et al., 2007). The most commonly used formula is as follows:

$$
\mathrm{BV}=\pi / 3 \times \mathrm{MP}^{2} \times(\mathrm{MR}+\mathrm{LR}+\mathrm{IR}-\mathrm{MP})
$$

Where, BV is the breast volume, MP is the mammary projection, MR is the medial breast radius, LR is the lateral breast radius, and IR is the inferior breast radius (Kayar et al., 2011). It is suggested that the measurements should be performed when the participant is in a sitting or standing with her arms at her sides. The advantages of anthropometric measurement methods are its feasibility, easy access and its very low costs (Kovacs et al., 2007; McGhee \& Steele, 2011). However, this measurement method is highly variable and is dependent upon the individual observer implementing the measuring (Bulstrode et al., 2001; McGhee \& Steele, 2011; Kovacs et al., 2007; Simmons \& Istook, 2003; Yip \& Yu, 2006). This includes imprecision in landmark location, subject positioning and instrument applications (Simmons \& Istook, 2003). McGhee \& Steele (2011) further substantiates this by explaining that the complexity of breasts makes no one parameter or mathematical equation standard enough for all breast sizes and shapes.

\section{Water-displacement techniques}

Water-displacement techniques (also known as Archimedes Principle) involve the use of a calibrated container. To measure the volume of the breasts, each breast is placed into the container filled with water. The volume of the water is then displaced and measured (McGhee \& Steele, 2011; Yip \& Yu, 2006). For the Bulstrode et al. (2001) study, this technique resulted in overall majority favorability among participants. However, the study determined that it is not the best form of measurement as participants had difficulty in performing the test adequately. Water-displacement techniques also have limitations in the breast volume (e.g., very small breasts) they are able to measure, as well as not fully accounting for the variability in individual breast shapes (Bulstrode et al., 2001; McGhee \& Steele, 2011). The McGhee \& Steele (2011) study concludes that breast volumes for smaller breasts tended to be underestimated because of the difficulty to be fully immersed in the water displacement beaker.

\section{Thermoplastic moulding}

Thermoplastic moulding is recognized as another breast volume measuring technique (Bulstrode et al., 2001; Kovacs et al. 2007), which involves a physical 3D mould of each individual breast. However, the inflexibility of the thermoplastic material used for moulding cannot perfectly model the breast form and becomes easily distorted under any manual pressure (Kovacs et al., 2007). Bulstrode et al. (2001), Kovacs et al. (2007), and Lee et al. (2004) all agree that this technique is particularly convenient and economical. But, since the mould separates the chest from the breast, the actual curvature of the breast wall is not considered and therefore the breast volume calculation can be misleadingly small (Kovacs et al., 2007; Lee et al., 2004). Consequently, cast moulding is deemed as the least accurate in comparison to mammograms, waterdisplacement and anthropometric measurements (Kayar et al., 2011). 


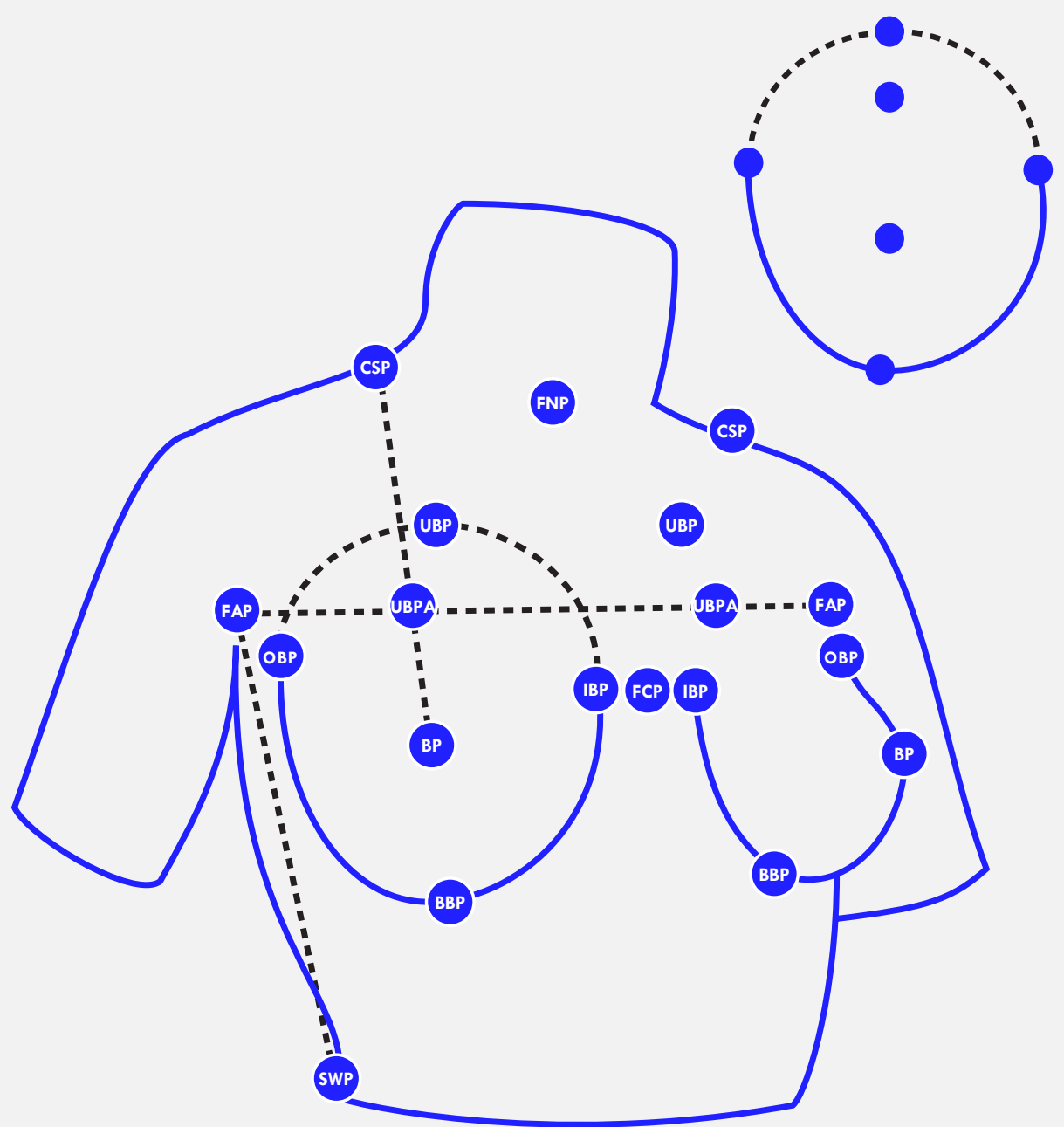

L A N D M A R K I N G P O I N T S \& BREASTOU T L I N E

$\stackrel{\vec{D}}{ }$ 


\section{Breast outline}

In order to fully understand breast characteristics for bra design and fit, breast volume data, though highly significant, cannot be the only metric. Previous research (Lee et al., 2004) stresses the importance of including shape and outline of the breast in measurement protocols. The breast outline is the curve that defines the boundaries of the breast. McGhee and Steele (2011) suggest that breast shape and outline may be as important as breast volume. The study determined that a range of breast volumes could be attributed to one bra size, highlighting limitations of traditional sizing system. There have been multiple ways of understating breast outline. Some researchers have measured the breast outline by pulling the breasts in superior, medial, and lateral directions in their palms (Lee et al., 2004). The outer crease line of the breast has been determined by pulling the breast in the lateral direction and the inner crease line was determined by pulling the breast in the medial direction (Lee \& Hong, 2006). However, this process of manually obtaining measurements of the outline of the breasts requires a repeated pull and push of the breast tissue. Also, this manual method can be both uncomfortable for the participants and difficult to measure. Alternatively, $3 \mathrm{D}$ scanning has been suggested as an efficient way to understand breast shape. This method allows for analyzing the $3 \mathrm{D}$ breast model, which could provide more information from the 3D surface point cloud data (Chen \& Wang, 2015; Yip \& Yu, 2006). However, reliable breast outline is difficult to define due to breast sag of an unsupported breast (Lee et al., 2004). Lee \& Hong (2006) suggest measuring the breast while wearing a bra made of thin elastic fabric rather than measuring the nude breast accounting for breast sagging. However, any additional fabric or clothing risk deforming the breasts and can impact the resulting measurements.

Anatomical landmarks have been suggested to aid with obtaining accurate breast outline. However obtaining accurate landmarks, specifically the under breast curve area, is difficult, again due to unsupported breast sag (Lee et al., 2004), and attempts to obtain automatic landmarking from 3D scanners did not work consistently on all body types (Simmons \& Istook, 2003). Therefore, establishing visible landmarks before the $3 \mathrm{D}$ scan, can help the data be widely understood. As a part of their study, Lee et al. (2004) provided new shape parameters of breasts for the design process of comfortable and formfitting bras. Landmarks and anatomical reference points include: Upper breast point (UBP), upper breast point A (UBPA), center shoulder point (CSP), the bust point (BP), front armpit points (FAP), inner breast point (IBP), outer breast point $(\mathrm{OBP})$, side waist point (SWP), the bottom breast point (BBP), front center point (FCP), and front neck point (FNP). These points help establish a clear understanding of how to reference the breast (discussed in detail in Chapter 7) and will be vital in developing accurate measurements and designing adequate bra fit (figure 2.9.).

\section{"...a range of breast volumes could be attributed to one bra size, highlighting limitations of traditional sizing system"}




\section{Body positions \& relationship to bra fit}

Chen et al. (2010) offer a quantitative approach to validate body variation, specifically looking at the relationships between shoulder slope, bust prominence, back curvature, and acromion placement, and influence on poor bra support and bra-motion restriction. In their study, they used a 3D body scanning system to extract angle data for the shoulder slope, bust and back and related the data to bra fit problems, including poor bra support and bramotion restriction. The study concluded that significant relationships existed between the figure variations and poor bra support, as well as, figure variations and bramotion restriction. Results suggested that relationships exist between figure variations of shoulder slope, bust prominence, back curvature, and acromion placement (Chen et al., 2010). The physical categories of shoulder slope, bust prominence, back curvature and acromion placement have effects on not only bra support, but also bra motion restriction. The variability in body shape, specifically through these four physical categories need to be accurately measured and analyzed in order to influence bra design and fit. The majority of the problem were found in bra support for body types with a relationship between large bust prominence and backward leaning acromion placement (Chen et al., 2010). Manufacturers and retailers are thus encouraged to use angle variable of bust prominence and acromion placement to predict consumers' perceptions of bra support. This new figurevariation combination could lead to improved bra designs, especially for those with larger breasts.

\section{Breast asymmetry}

Breast symmetry is rather uncommon. In fact, breast asymmetry has been reported from $62 \%$ of the female population (Losken et al., 2005) to upwards of $82 \%$ (Gabriel et al., 2011), with the left breast often being larger than the right (Losken et al., 2005; Page \& Steele, 1999). It is important to note that breast asymmetry may not always mean the difference in breast protrusion or volume, however breast asymmetry can be defined by the differences in breast outline for a women, all which influence negative bra fit.

It is not surprising to note all bras are designed and manufactured based on breast symmetry (Hardaker \& Fozzard, 1997), which means that both cups and underwires are exactly the same. This naturally results in fitting problems for women with even the slightest asymmetrical breast shapes. Majority of bra fit recommendations suggest that the bra should be fitted to the larger breast, which means that any asymmetry may reduce the effectiveness of the support of the bra for the smaller breast. Mills et al. (2015) suggests that due to the potential differences in bilateral breast mass due to asymmetry, a researcher's recommendations for bra design may depend upon the researcher's decision to analyse the left or right breast. Mill et al. (2015) research explored breast movement and displacement in breasts, and concluded that breast movement asymmetry does occur which may be linked to the reported differences in breast size and density. Furthermore, asymmetrical breast movement will have implications on bra design and in turn, fit, as the direction in which the greatest breast displacement occurred differed depending upon right or left breast selection (Mills et al., 2015).

The question is whether bra manufacturers could develop asymmetrical cups or customisable bra cups to minimize the displacement of each breast individually (Mills et al., 2015). Additionally, the question of how bra wearers themselves regard breast asymmetry and how they would respond to asymmetrical bra design is also important. Mills et al. (2015) recommend bra manufacturers to look into the practicality of producing asymmetrical cups that can cater for "all combinations and magnitudes of breast asymmetry". 


\section{SECTION IV.}

\section{BRA PRODUCTION}

A major point of dissatisfaction for bra wearers is the actual design of bras (Bye \& LaBat, 2006), especially for those women with larger breasts who have concerns with limited options (McGhee \& Steele, 2010). Design is one of the top three concerns during fitting sessions (Bye \& LaBat, 2006). There are various different types and styles of bras (figure 2.10), and thus fit requirement for each style and design is unique. For example, sport bras are designed to control breast motion (McGhee \& Steele, 2010; White et al., 2009) with little regard to breast shape and contour. In contrast, the socalled everyday bras have a deeper interest on body shape and appearance.

Very little research has been conducted on the bra design process (Hardaker \& Fozzard, 1997; Mitchell, 2013). The commercial process relies heavily on the expertise and heuristics of the designers (Hardaker \& Fozzard, 1997, p. 113), yet there is limited analysis on this process (Fong, 2014). Additionally, there is very little academic research on analysis of the design process of the bra, and defining the design elements and procedures for a "perfect" bra. Thus, there is a lack of reliable data of how the design influences the fit of a bra. The Hardaker \& Fozzard (1997) study on the bra design process marks as one of the few readily cited research pieces. They examined the working methods of professional bra designers and the manufacturing process of bra design by comparing different companies. 
01 Adhesive

Usually made of silicone, polyurethane, or similar material, they are attached to the underside of the breasts using medical-grade adhesive. Some versions provide one piece for each breast. May be reused for a limited number of times and provides little support.

\section{Balconetie}

Sometimes known as a shelf bra. Lifts the breasts to enhance their appearance, shape, and cleavage. They may be either wire-free or underwired.

\section{Bralefte}

A lightweight, simple design, usually an unlined, soft-cup pullover style bra. The breasts are covered but the bra offers little, if any, real support and is suitable for small busts. Usually wire-free.

\section{Convertible}

The bra straps can be detached and rearranged in different ways. Some strap arrangements include straight, criss-cross, halter, strapless and one-shoulder. They may be either wirefree or underwired.

\section{Demi-cup}

A partial-cup bra style that covers from half to threequarters of the breast and creates cleavage and uplift. The straps usually attach at the outer edge of the cup. Usually contains an underwire.

\section{Front-closure}

Bras with a clasp positioned in the centre front gore between the breasts. Frontclosure only is defined by the front band style, the rest of the bra design may vary. They may be either wire-free or underwired.

\section{Full-cup}

Designed to offer maximum support and coverage for the entire breast. A practical design for largebusted women. Full-support bras provide lift and support via clever design details such as seamed cups and detailed side panels. Usually contains an underwire.

\section{$08 \mathrm{Knitfed}$}

\section{( $s$ eamless)}

Machine knitted in one piece with few or no seams. Usually unstructured and similar shape to a bralette. Usually wire-free.

\section{Padded}

The lining of the cups are thickened and enhanced with foam padding. Unlike push-up bras they are not intended to significantly increase cleavage. They may be either wire-free or underwired.

\section{Plunge}

Allows for lower cleavage. Designed with angled cups and an open and lowered front band. Unlike push-up bras, are not generally as heavily padded as many women with larger breasts wear them. Usually contains an underwire.

\section{Push-up}

Designed to create the appearance of increased cleavage. Uses angled cups containing padding that pushes the breasts inwards and upwards. Usually contains an underwire.

\section{Moulded}

They have seamless, preformed cups containing a foam or other lining that helps define and hold the cup's shape, even when not being worn. Usually contains an underwire.

\section{Long-line}

Designed with an extended band, usually reaching down 2 or 3 " beyond the bust line. Most have seams to maintain structure. They may be either wire-free or underwired.

\section{Recerback}

Designed with shoulder straps that form a " $\vee$ " or "T" pattern between the shoulder blades. Many sports bras use a racerback design to improve support and reduce bounce. They may be either wire-free or underwired.

\section{Sports}

Designed for athletic activities to provide firm support and minimize breast movement during exercise. Various designs are suitable for a range of exercise, ranging from yoga to running. Usually made of stretchable, absorbent fabric like Lycra, and designed to wick perspiration from the skin to reduce irritation. They may be either wire-free or underwired.

\section{Strapless}

A design that has no straps and therfore relies on an extra-wide band for breast support. Some convertible bras (see above) allow straps to be removed, making a strapless bra. They may be either wirefree or underwired. 


\section{BREAST STYLE DIRECTORY}
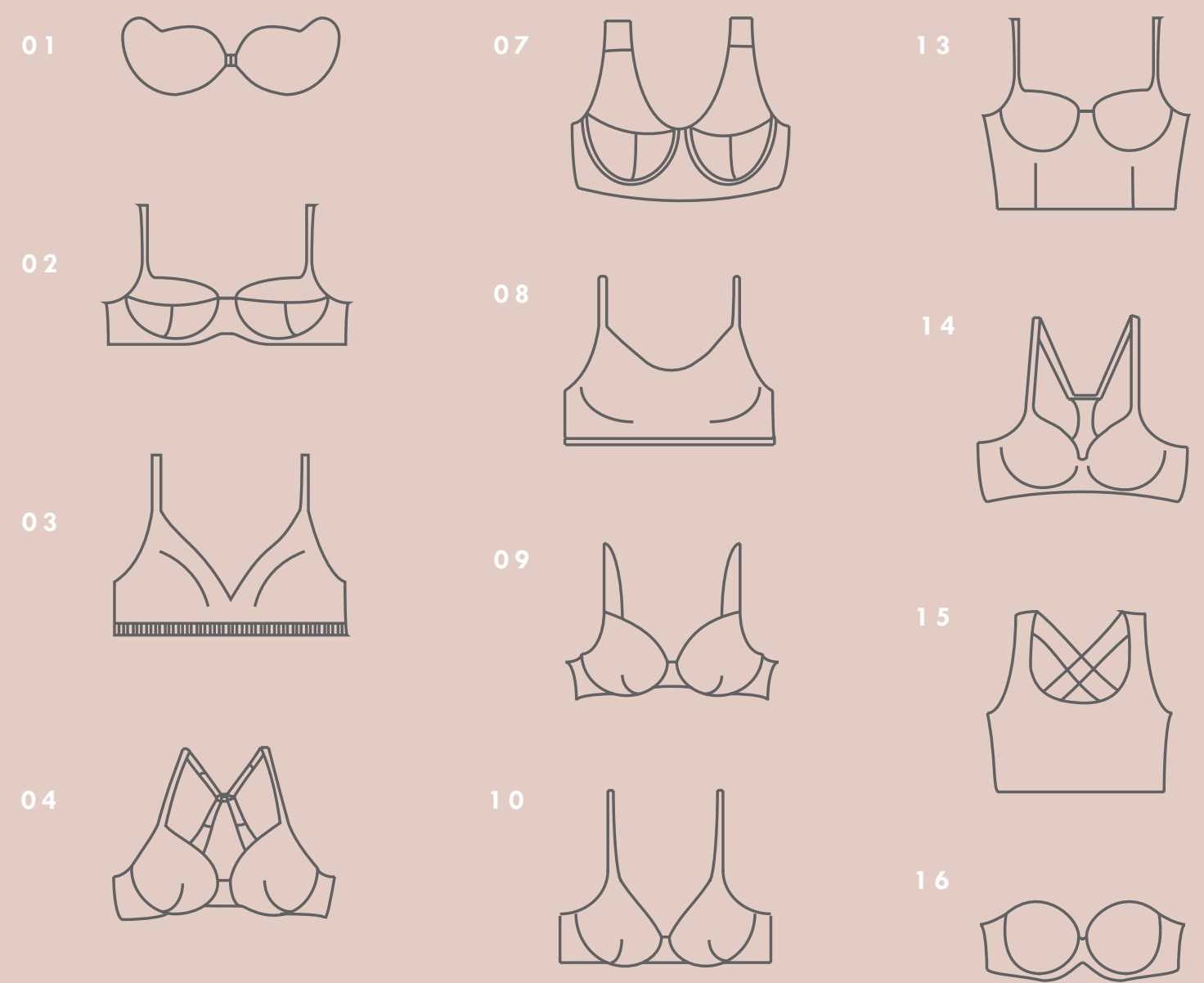

03
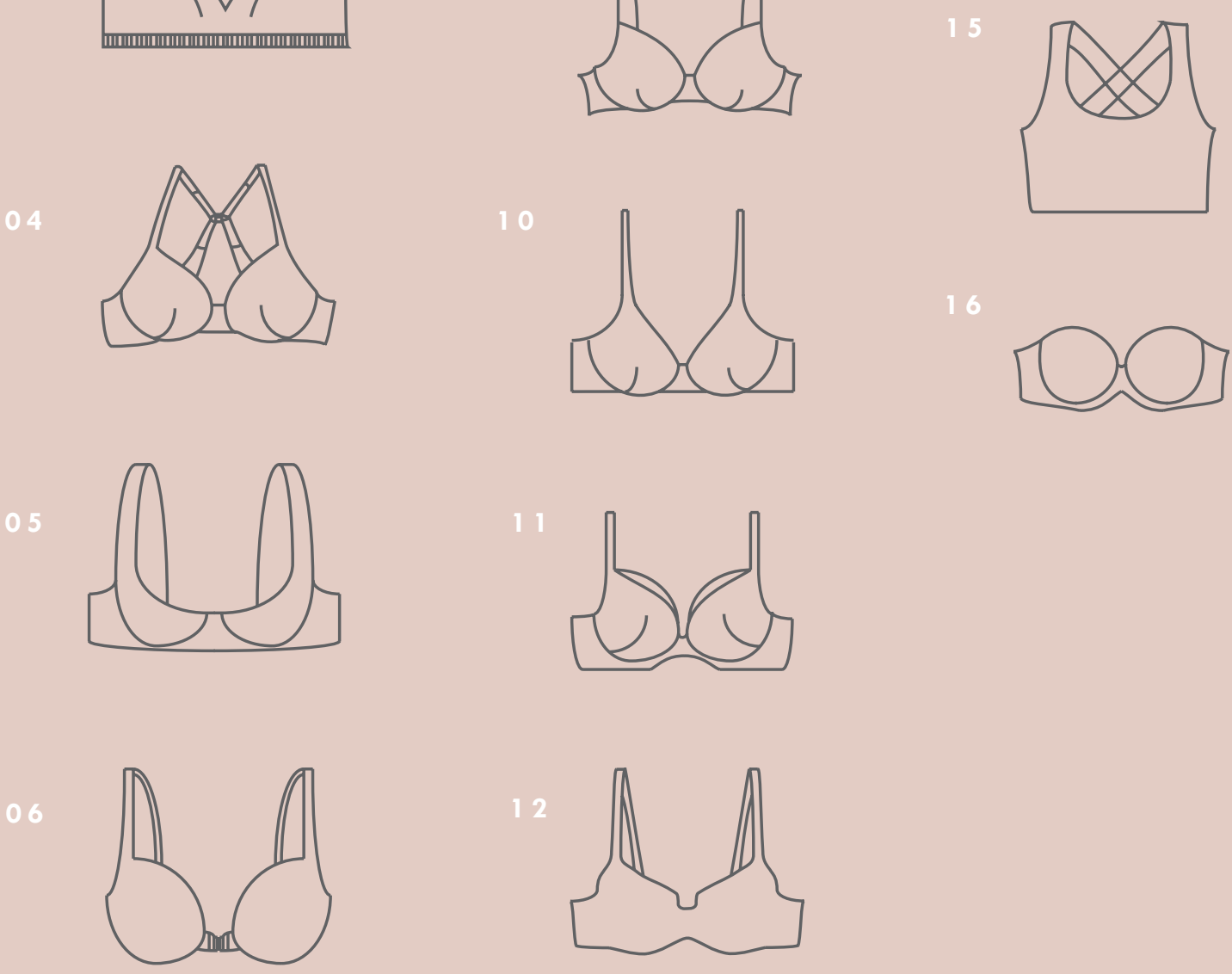


\section{The underwire}

As mentioned earlier, there are several intricate parts of the bra, each with an important function to the fit and design of the bra. Whether a bra is suitable for a woman depends not only on the bust and underbust, but also on the shape of under wires (Wang et al., 2009). Although the underwire appeared as early as 1934 , it only became common after World War II (Wohleber, 2003). Currently, the underwire can be seen in the majority of the bras available because the underwire is used to support the lower part of the breasts and also shapes the breasts (Wang et al., 2009). Therefore, this thesis will pay special attention to the underwire as a pivotal part of the bra and bra design. The underwire in bras has been a source of irritation for some women perhaps because this part of the bra is designed less for fit and comfort and instead for just shape and aesthetic. It has even been reported that the underwire has been one of the most important design factors causing discomfort (Kim et al., 2000). The problem become even more acute if women wear an ill-fit bra, as the underwire will press against their galactophores, blood vessels, nerves, lymphatic and other organs, and long term pressure of the underwire will lead to pathological changes of the breasts such as hyperplasia of mammary glands (Wang et al., 2009). A main cause of this unwanted pressure is the incorrect measurement of breasts (Yip \& Yu, 2006).

The body's rib cage has a curvature to which the underwire must conform. However, the underwire must also support the weight of the breasts, so there is the conflict. That is, the underwire must be both flexible for comfort when the body moves, and rigid for structural support at the same time. The main materials of the underwire are two-dimensional, steel, rigid plastic or other materials that are not easily deformed. In order to support the breast comfortably, some manufacturers have developed new underwire bras. As early as 1993 , Wacoal Company created a curved underwire based on changing the curvature of the normal bra, using steel or shape memory alloy. They claimed that the shape memory alloy could help the underwire bra prevent shape changing or could resume to its original shape after shape changing (Rong, 2006). In 2001, Wacoal developed a curvilinear underwire by using nickel-Ti alloy and a stainless steel underwire, which changes the cross-section into an elliptical shape. Both examples demonstrate the need for alternative underwire forms and materials to be explored, however both examples remain designing with the use of metal and stiff material properties. There is an opportunity to explore desired structural support meanwhile a much softer and flexible outer layer for comfort and mobility with the body.

\section{Pattern development}

All the bra styles and functions can be compartmentalized in three different design processes: cut and sew, moulded, and seamless. All bra design models have been proven to involve a lengthy process requiring a combination of design creativity, precision for measurements and detailed knowledge of fabric performance (Hardaker \& Fozzard, 1997).

The majority of bra designs are based off the "cut and sew" method (Hardaker \& Fozzard, 1997; Yip \& Yu, 2006) involving developing precise pattern drafting, which are composed of flat panels and shaped to the nearest millimeter (Hardaker \& Fozzard, 1997). The garments are constructed from $2 \mathrm{D}$ pattern design, then sew into garments and fitted to a life model. The designer will then begin a prototyping and adjustment loop to which the design will check fit and support and amend the pattern accordingly. Once the designer is satisfied with the fit, the pattern is then graded to a variety of sizes. In their study based of ten bra companies, Hardaker \& Fozzard (1997) concluded that this loop or "trial and error" has been noted as a main feature of the pattern development phase. All "cut and sew" bra designs involve some form of pattern drafting. The three main pattern-making methods used by apparel designers in the industry are, draping, direct pattern drafting and flat or block pattern drafting. Draping method involves creating pattern pieces by applying fabric directly onto the three dimensional form. The advantage is that the designer can transfer their two-dimensional sketch to a threedimensional sample directly onto the form (Shin, 2009).

However, finding fabrics with an equivalent drape to a fabric for a finished garment can be difficult, costly and not appropriate for a commercial or mass production level (Shin, 2009; Yip \& Yu, 2006). Direct pattern drafting is a method that develops a flat $2 \mathrm{D}$ pattern by "freehand drawing" based on direct body measurements (Yip \& Yu, 2006). To develop the formula, the designer must have a good understanding on the relationship between the developing pattern and the corresponding body measurements. This method requires detailed knowledge of the body (Yip \& Yu, 2006) and requires a high degree manual experience. Moreover, due to the level of dependency to the body measurements, which are obtained manually, this method seems weary to human error. The last method is based off of creating patterns by manipulating a basic block, which is like a "sample" pattern from which patterns for many different styles can be developed. The basic block is based off using a direct drafting method or a draping method. The basic block will vary from one company to the next (Shin, 2009). Flat patternmaking is known to bring consistency in both size and fit for mass-produced garments (Shin, 2009). This 
method is therefore the most favorable for fashion industry. However it can be very inefficient, and again requires a great detail of manual skill and expertise.

According to research conducted by Hardaker and Fozzard (1997), the average time spent on bra pattern development can be anything from two to six months, and the average training time expected for becoming a professional bra patternmaker capable of producing a good fit bra is 10 years. Additionally, these $2 \mathrm{D}$ pattern designs are based mainly on manual calculations, which require enormous of skill and experience, without such will produce an ill-fitted bra (Yip \& Yu, 2006). This is both inefficient and expensive and there is a clear industry need for more efficient methods of pattern making, which guarantee a good fit. There have been developments in the bra design development other than the traditional "cut and sew"; these include the moulded bra (discussed below) and the seamless bra (discussed in Section V.).

The moulded bra is distinct to the "cut and sew" bra in that instead of many flat pieces being stitched together, the moulded bra features a seamless rendered foam cup. In most cases the cup is made of polyurethane (PU) foam (Wu et al., 2012). The process involves a pair of aluminum male and female moulds with a conformed shape. The heated male mould stretches and compresses the flat foam sheets toward the female mould and a high temperature (Wu et al., 2012). The bra cup moulding can be completed with a variety of PU foams with different densities and harnesses. This allows for a variety of shapes, support systems and styles (Wu et al., 2012). The sizing of the moulds depends on the bra companies that order the cups (Wu et al., 2012). The moulded bra represents a much more economical and convenient way of constructing a seamless 3D form for bra design (Yick et al., 2008). However, the moulded bras have been found to have limitations in shaping the breasts to a singular circular shape, due to the foam material, which is undesirable for some who prefer a more natural look or for those who have asymmetrical breasts (the foam padded does not stretch with the breast). The foam also is not made of a breathable material, which can lead to irritation and discomfort. Additionally, the moulded foam bras are limited in supporting women with larger breasts. The shapes of the cups are determined in the moulding process and sometimes cannot account for heavy breast weight and hence the breast shape becomes compromised and some sagging may occur.

\section{The role of CAD}

Over last couple decades, computer-aided design (CAD) systems have been used for pattern amendment and grading to establish a more productive and efficient way to design bras. Hardaker and Fozzard (1997) acknowledged that almost all the companies surveyed had some type of CAD system. However, CAD was not being used to design the first pattern, this was still done manually by hand. Instead the CAD systems were being used for later pattern drafting and especially used for grading (Yip \& Yu, 2006). CAD systems are being used for shortening the product development cycle by improving pattern accuracy and grading sampling (Yip $\& \mathrm{Yu}, 2006)$. They can also be used to help designers visualize how the bra will look on the screen before they are created. Most CAD systems for bra design still maintain a pattern approach to bra design development. For example, a bra cup is represented as several flat pieces that must be sewn together (Yick et al., 2008). A system that "incorporated 3D working methods and addressed the idiosyncrasies of the bra fitting process" is regarded as an important requirement for future CAD systems (Hardaker \& Fozzard,1997). CAD offers an opportunity for more accurate approach to sizing and measuring as well as being able to draft a design on the computer before a physical form as such to reduce material waste. Additionally, CAD systems offer a storage of digital data which allow the designer to issue faster adjustments and easier prototypes. However, there is a clear need for updated CAD systems that offer an alternative way to create bra design forms not based on the manual pattern making approach.

\section{Materiality}

The secrecy of the bra design process has led to limited shared knowledge. Many of the key design processes, such as specific pattern techniques and measuring are kept within the company. The same proprietary attitude is also seen with material and fabric selection. The choice of fabric will usually influence the measuring of the pattern and ultimately the shaping of the design. Therefore materiality is usually decided at the same time as concept drafting. Materiality is an important piece of the design, both in quality and sustainability. In the case of bras, materials play a major role in how much impact or pressure is on the body and influences support and comfort, two key functions of the bra. A major part of the bra is the aesthetic feature so much so that some women solely base their choice off this. However, the bra must also perform the difficult role of providing adequate support for the breasts and thus this will impact its aesthetic and material. 


\section{ADVANCED TECHNOLOGIES}

Though many advanced technologies have been readily adopted in industry and research for decades, the rise in accessibility and drop in prices have also allowed new technologies to have a positive impact on the development and design in other areas that have traditionally avoided these processes. Literature has pointed to the potential of $3 \mathrm{D}$ scanner to gather breast volume, shape, three-dimensional curvature, breast outline, and many more measurements needed for bra design. Additionally, given the variability of breast shape and sizes and how pattern making is quite difficult to cater for this variability, there is a need to find manufacturing techniques that allow for individualisation: 3D printing and 3D knitting. Below is a list of three advanced technologies: 3D scanning, 3D printing and 3D knitting, which are crucial to explore for this thesis project. 


\section{D scanning}

The $3 \mathrm{D}$ scanner, as the name implies, is a device that scans real-world objects or environment, and then analyses the data collected in 3D form (Ebrahim, 2015). It is non-contact (i.e., the object does not need to touch the scanner), fast and accurate imaging tool. The 3D scanner has the ability to extract infinite number of data types and measurements (i.e., only constrained by the computer memory), as well as shapes and angles (Simmons \& Istook, 2003). The primary types of scanning systems are based on laser and light (Kim et al., 2014), and were developed during the last half of the 20 th century (Ebrahim, 2015). The 3D scanner has been applied to many different spheres of knowledge, from accumulating data for civil engineering for building analysis to medical field. Given the qualities and potential of this technology to address the criteria needed for the project, it has been decided to investigate its potential for the purpose of body imaging for a better fit bra design. The use of 3D scanning to generate body models has many uses such as "3D shape data for avatar creation for online shopping, virtual fit trials, 3D product development, body dimension analysis for target markets, animation and graphics, health and fitness management" (Apeagyei, 2010). Additionally, 3D scanning for body imaging allows for mass customization. The emergence of 3D scanning for body imaging, and their accompanying integrated CAD systems have permitted "made-to-measure" as a viable marketing tool (Devarajan \& Istook, 2004). In a sense, this type of scanning is shifting mass production to mass customisation, where the measurement procedure would be carried out once, and allow future re-use and adjustment of stored data (Apeagyei, 2010). This allows for individualised sizing and design with faster turnaround times. The London company, Bodi.me, founded in 2013, offers an online shopping sizing solution. Where 3D scanners are used to measure the body and then tell the customer which sizes in which clothing labels will work for them.

3D scanning has been validated as an effective method for breast imaging. Unlike other breast imaging techniques, the $3 \mathrm{D}$ scanner has the ability to create accurate surface images of the complicated breast contour. The scanner is able to capture a $3 \mathrm{D}$ breast models, which can be later analysed to obtain the necessary measurements for bra design. Apparel researchers are relying more and more on body scan technology to overcome the disadvantages of manual anthropometry (Kim et al., 2014), such as human error. One of the most ideal aspects of using 3D scanning for body imaging is its ability to store data for 3D models, where it allows for access to additional body measurement data without recalling a person back (Shin, 2007).

Additionally more accurate information can be obtained from 3D surface point clouds (Yip \& Yu, 2006). Accurate sets of complex data can be produced and reproduced in seconds, and then mapped onto other body data to check for generalisability (Shin, 2007; Paquette, 1996).

There are some limitations to $3 \mathrm{D}$ scanning, which must be understood when using it as a breast imaging tool. In terms of breast imaging, wearing a bra, or a thin layer will distort the breast image and change the measurements of the breasts (Kim et al., 2014). Additionally, since the breasts are made up of soft tissue, it is important to understand how the breast moves. Scanning in multiple positions will allow for greater understanding of breast measurements and positioning.

Overall, 3D scanning has been found to represent a non-invasive method for breast measurement, which is comparable to the accuracy of classical techniques. 3D scanning also presents an alternative for quantitative evaluation of bust symmetry, shape, contour and distance measurements for bra fit analysis (Mitchell, 2013).

Therefore, through 3D scanning almost the parameters needed for breast measurement for bra fit and design can be acquired. 


\section{D printing}

The $3 \mathrm{D}$ printing process can be simply compared to the traditional inkjet printing method, but instead of ink the printer builds up the output layer by layer with different filament depending on the printer. $3 \mathrm{D}$ printing is also referred to as additive manufacturing, since, unlike other processes that cut or mould an object, the 3D printer will build up the object only using the material required. There are several different types of $3 \mathrm{D}$ printers that are defined by material being used and printing properties. Some include, Fused Deposition Modelling (FDM), Polyjet Photopolymerization, Selective Laser Sintering (SLS), Stereolithography (SLA), and Laminated Object Manufacturing (LOM). 3D printing technology first came about in 1984 and since then has been made readily commercially available. Furthermore, not only does 3D printing offer the ability to develop intricate and complex form, the process also enables the chance to develop fully customisable forms based off designs developed off 3D models like those obtained through $3 \mathrm{D}$ scanning. In terms of bra design, there is a possibility to use $3 \mathrm{D}$ printing technologies to rapidly produce customisable prototypes of bra components, such as the underwire.

\section{D knitting}

Knitting is defined as a process of forming a fabric by the intermeshing of loops of yarn or the technique of "constructing textile structures by forming a continuous length of yarn into columns of vertically intermeshed loops" (Spencer, 2001, p. 7).

3D knitting is essentially a set of individual strands of yarn that feed into a complex machine that manipulates small knitting needles. Each thread is controlled by its own machine head, which is capable of creating most any known knit pattern. 3D knitting allows for the capability to knit using various types of yarns including synthetic, cotton, wool and silk. Combining these yarns with elastomeric fibers, which can be knitted in, floated, or laid in (Bremner, 2005). This allows for designers to create their own fabric. 3D knitting has the potential for the designs to be knit without any additionally sewing or seams, for instance a full sweater can be manufactured without the need of sewing the sleeves to the body of the garment. It is claimed that this technology could shorten manufacturing processes, reduce material wastage, and offer greater comfort and better fit to wearers (Lam, 2005). 3D knitting has the potential to have outstanding physical, thermal, and mechanical properties, such as light weight, high stiffness, strength, and dimensional stability (Hu, 2008). This type of knitting is used in advanced structural applications such as in the aerospace, automobile and medical industries (Chen, 2015). With 3D knitting textiles and knitted structures could be formed very fine, intricate and complex (Spencer, 2001).

\section{Seamless or whole garment knitting}

3D knitting has been readily used in bra production through developing the seamless knitted bra. This process uses whole garment technology to knit in a circular motion to build up the bra design. There are no hooks or adjustable features; instead the bra consists of a pullover one piece of stretch fabric (figure 2.11.). Seamless knitting has already had numerous applications from underwear, to swimwear, nightwear, outerwear and medical-wear.

Potential benefits of seamless knitting include reducing the number of parts (43 components) needed in a typical bra pattern (Bressler et al., 1998) to a singular form that can remain in digital form from the designer to the manufacturer (Fong, 2014). Seamless bras are suggested to have a soft handle, are wire-free, easy fitting and therefore very comfortable to wear without the constraints, swellings or unsightly stitching that may be harmful to women's health (Rong, 2006). Zheng et al. (2009) conducted pressure evaluation of seamless knitted bras compared with conventional underwire bras. This study was the first attempt to use knitting parameters to predict pressure distribution of seamless knitted bras. They concluded that the seamless knitted bras showed significantly lower pressure, especially at the points of inner wire, outer wire and side bottom band than conventional underwire bras. This study also provided design parameters for the future development of seamless knitted bras in terms of loop length such as using longer loop length in the bra cup region, and increases the cup volume without compressing the breasts (Zheng et al., 2009).

However, the compression, or flattening, of the breasts is identified as one of the most critical limitations to seamless knitted bras due to the lack of 3D form and 3D structure (Fong, 2014; Jin et al., 2009; Rong, 2006; Zheng et al., 2009). As a consequence, seamless knitted bras are not considered favourable for everyday bra use, which can be compared to sports bras where the compression of the breasts is not regarded as adverse. Another limitation is that seamless bras currently on the market are not completely seam-free and some sewing is still involved (Fong, 2014). The lateral seams of the bra are eliminated, but trimming and cutting is still required for the fabric area outside the garment piece. Sewing is needed for creating clean forms around the neckline and the joining shoulder straps (Fong, 2014). Currently, commercial seamless knitted bras have focused on flexibility, softness and smoothness as features of knitting properties. Very few attempts have been conducted into developing new forms or wires to support the breasts naturally, which are also suitable for knitting performance (Rong, 2006). A 3D knitted bra with knitted complexities such as different knit types, slots for wires and different types of closures and hooks could be a possible solution and has been addressed as part of this research (Chapter 08). 


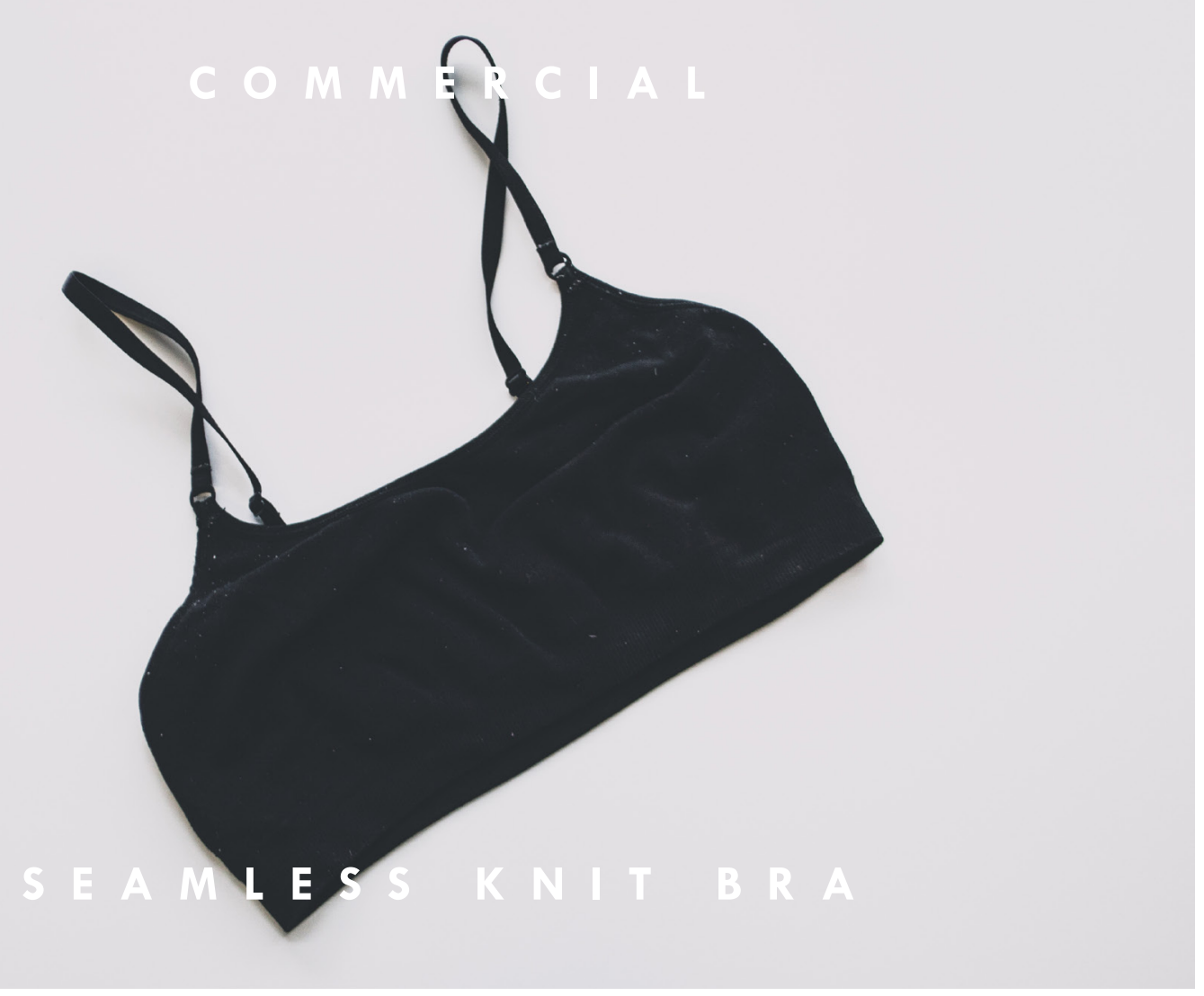

It offers the same favorable aspect as the seamless bra, but it can possibly address traditional seamless bra's limitations such as responding to breast compression and flattening by the addition of an underwire. Finally, it can offer a more contemporary aesthetic appeal.

Zheng et al. (2006) offers the following suggestions on the design of seamless knitting for bra design accumulating structure, all of which are addressed in this research (Chapter 08):

- Using longer loop length in the cup region can increase the cup volume without compressing the breasts.

- A denser knitting structure should be applied to create the required rigidity at the side panel to hold the breast shape.

- Elastic yarn tension should be carefully selected to provide appropriate underband tension to hold the bra in place without excessive pressure.

- Soft 3D supporting wire is recommended to support the breast weight in a natural manner and used to reduce the pressure at the cup base point. 
CHAPTER 02

\section{BACKGROUND}

DISCUSSION

\section{Section I. User Experience}

Innovative approaches have been made to by-pass in person bra-fitters. However an abundance of flaws are common due to inaccurate self-reporting as well as these innovative approaches still not responding to the vast complexity of the breast shape and measurements needed for bra design. In this section, user's general experience with wearing current design of bras was discussed, in particular the difficulty of finding the right fit and comfort for one's particular shape and size. This problem is compounded because of lack of access to professionals who may be able to accurately assess size and type. The research supports more self-assessment but individual's own poor training and education on how to evaluate the problem combined with certain sensitivity of this subject has made the progress in this area slow and ineffective. Technology certainly can help and online shopping with supportive questionnaires has provided an avenue for a more private self-assessment. However, as shown above, these on-line processed, despite their good intentions, have not necessarily provided the right answers for many women. The research hopes to respond to these limitations encountered from the online quizzes by providing a measuring system that offers a much more comprehensive measurement catalog, while not being dependent on self-reporting.

\section{Section II. Size \& Fit}

Bra sizing and fitting has been part of bra selection almost as long as modern bras were invented more than a century ago. However, whether because of cultural constraints and sensitivity or women's own lack of understanding about breasts and bras, the science and industry of bra design and manufacturing have relied on two basic metrics: bust girth and cup size. In this system, women's breasts are divided into a discrete alphabetised system. This is despite the fact that women's breast size is a continuum across the population, and even more specifically a woman's left and right breast may not be the same size or shape. The system in place was created when the average breast size was B and has stayed the same even though the breast sizes have shifted upward towards the average of $\mathrm{D}$ (though designing to average alone is not adequate either). It is clear that traditional bra fitting methods are not adequate if comfort and health are also included to basic measures (e.g. 12B) and aesthetic. Although there are professional bra fitters, lack of standardised training and inconsistency in methodology have found them lacking. There have been attempts to create set of criteria to more accurately measure breasts, but they all lack the fundamental process to measure individual measure of comfort. Other researchers have proposed new methods of breast measurement and to establish new sizing charts. However, these proposals are still 
currently based on linear correlations and do not consider 3D breast shape, angle and profile. Some studies have shown individuals can through process of elimination find the "right" size bra, but most consumers lack education in assessing what is the "right" fit bra.

\section{Section III. Breast Measurement}

It may not be surprising to note women may experience feelings of embarrassment and self-consciousness when purchasing bras from people conducting the sizing. Manual measurement methods, such as anthropometric measurement techniques, not only tend to be the most uncomfortable but also have a poor record of accuracy. Similar problems exist in water-displacement and thermoplastic cast moulding. It has been verified that out of the computer-analyzed measuring methods, applying $3 \mathrm{D}$ breast data through $3 \mathrm{D}$ scanning measuring techniques would be the most beneficial for bra design and fitting. 3D scanning represents a non-invasive method of breast measurement extraction, which can be comparable to the accuracy of other methods. 3D scanning and corresponding software allows for accurate breast volume data. In addition, unlike other types of measuring techniques, 3D scanning allows for the opportunity of quickly and accurately extracting breast and body surface measurements and angles. This method can be used to help determine body shape, posture, contour and body distance measurements, which are important features to influence bra design and bra fit. In contrast to manual measurement methods, 3D scanning allows for digital models of the breasts, which can be stored and analyzed. This may limit any potential discomfort for the bra consumer during measurement. However, simultaneously, the willingness of the consumer to have their breasts scanned and this data stored digitally may also bring up issues of privacy.

Optical data of sagged breasts has not been fully applied to the design of bras (in underwire and cups). Thus determining accurate measurements for the breast outline is important. Pre-scan manual landmarking will allow for the inframammary fold to be visible and marked. The 3D scan will locate the landmarks, which will aid in outlining the breast. In summary, there are various points of measurement to be understood for bra design and fit. A combination of 3D scanning and manual pre-scan landmarking will be key for this research to obtain breast volume, breast outline, body shape relationships, posture, breast asymmetry, body shape distances and more. Breast asymmetry is a necessary measure, and the cup design in bras that accounts for this asymmetry should be carefully considered.

\section{Section IV. Bra Production}

Until very recently there were little or no research on the design and manufacturing of bras with most of the manufacturers focusing on the aesthetic rather a "proper" fit. Perhaps one of the key innovations in bra design is the use of underwire. However, consequently and because of poor bra sizing, underwire bras have created an insufficient support structure. There is an opportunity for the bra to have technology that allows for customised measuring of breasts and well as customised manufacturing of bras. This exploration can ultimately aid in better fit bras at a mass level, and is something that traditional pattern-making has not explored at a nonbespoke level. Traditional pattern making for bra design has been the most commonly way to design and develop bras. However, there is more room for advancement in bra development process. The time consuming and intricacies in traditional pattern making techniques can be resolved through innovative processes and advanced technologies, the subject of the next section.

\section{Section V. Advanced Technologies}

Different advanced technologies currently play a role in bra development and experience. However, there is an opportunity to respond to the current limitations such as, 3D knitted bras lack of 3D form, or 3D printed underwires large and stiff materiality, and explore more with digital technologies and processes throughout the entire bra process. Seamless bra design and manufacturing could provide a partial solution to development of well-fitted bra. But like any process, it has its own limitation such as a lack of three-dimensional shape and structure. Advanced technologies could provide additional resources and harness the best aspect of manual design. This chapter discussed three of these technologies: 3D scanning, 3D printing and $3 \mathrm{D}$ knitting. 3D scanning provides a stress free, one-time data collection that provides a "true" image of one's body and provides data needed to addressed the required parameters for better bra design. 3D printing and 3D knitting could provide a resource for on-demand, body specific manufacturing of bras that are not only comfortable and supportive but also aesthetically pleasing. 

C H A P T E R 03

BRA SYSTEM 


\section{CURRENT BRA SYSTEM}

In the current bra system, the design and manufacturing of bras is a separate and somewhat independent process than purchasing and fitting the bra. In the first phase of the process, most bras are constructed from flat panels, which are then shaped to the $3 \mathrm{D}$ form and fitted to a life model. The designer will then check the fit and design, making any necessary adjustments. This is a start of a long prototyping cycle, which involves many iterations of fit and pattern amendments to achieve the desired bra design. When the bra sample is completed, it is then graded across the company dependent size range (i.e., each manufacturer sets its own size (see Chapter 02, Section II, for more information on bra sizing) (Hardaker \& Fozzard, 1997). When the graded patterns have been finalized, they are sent to production along with manufacturing instructions.

The purchasing and fitting stage of the current bra system includes shopping in a store or online with a range of styles, brands and sizes. The customer selects a bra with a size closest to her perceived size, and then must try it on and check the actual fit. The fitting can either be done independently or with the help of a "professional" bra fitter. This begins a familiar process of trial and error until the customer can find a bra that fits as best as possible (as explained in Chapter 02, Section II).
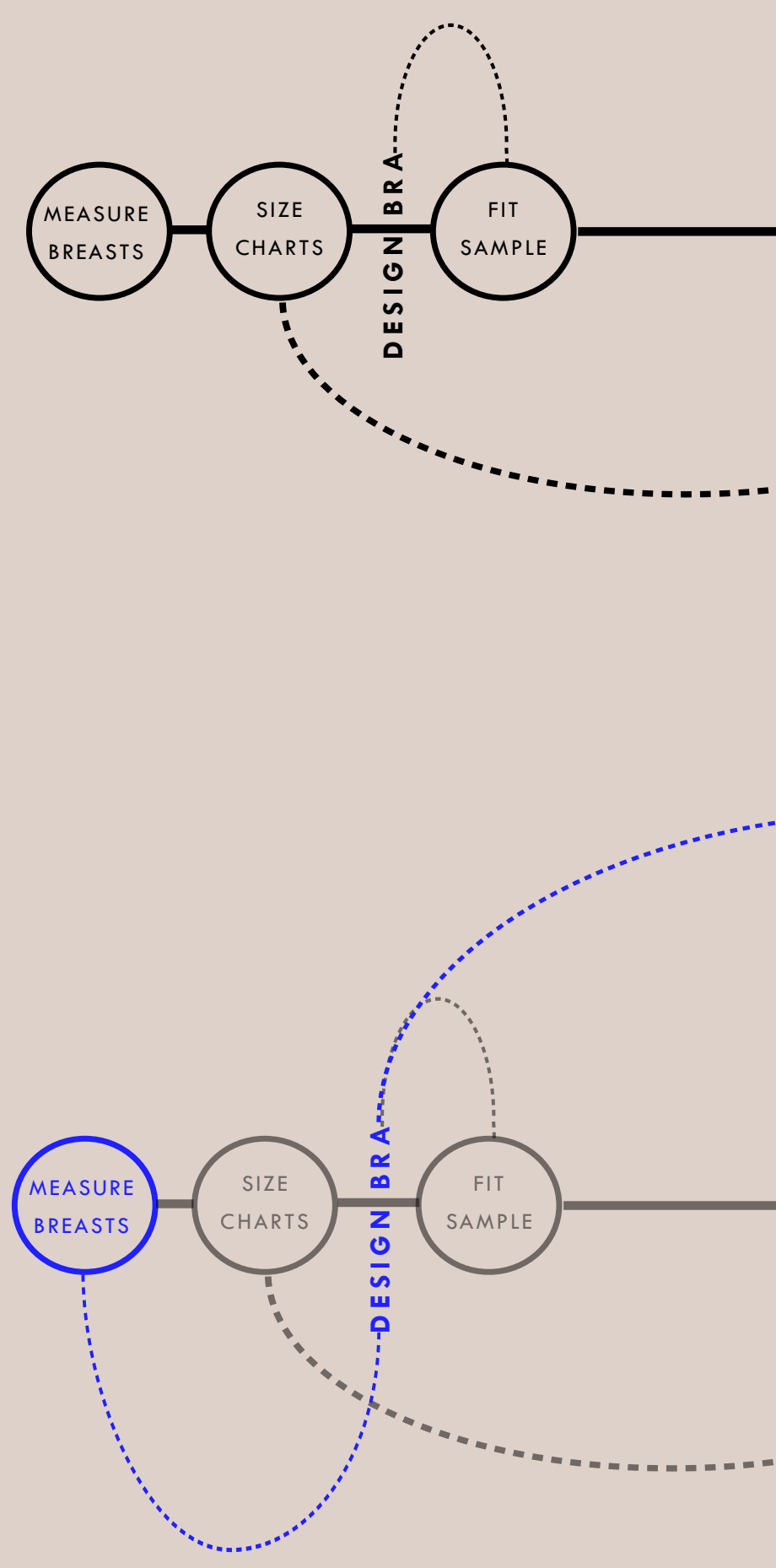


\section{Proposed System Framework Product-Service-System}

A product-service-system, the result of an innovation strategy, can be defined as shifts in the business focus from merely designing and selling products, to selling a system of products and services which are interrelated (Manzini \& Vezzoli, 2003). This integration between body of products, services and communications is a design model that addresses the changes in technological, social and consumer attitudes (Mont, 2002). The productservice-system model will be used to help influence and develop a framework for an innovative bra system in this thesis. For the consumer, a product-service-system shifts from merely buying a product to buying into a service that requires a higher level of customer involvement (Mont, 2002). For the producer, interests must be extended beyond the usual product life cycle boundaries and venture into the connections with other products and services, which results in an integrated solution for the consumer (Manzini \& Vezzoli, 2003). In this model, the relationship between consumer and producer does not end with the purchase of the product, instead the product is part of a system that is able to adapt and change with the consumer. This method can be applied to how bras are designed, fitted and purchased as well. The fit of the bra will no longer be an afterthought to the design. The design will incorporate and will be based on real data of the topology of each individual consumer. This data will then be stored and can be adjusted by the producer to better and more accurately serve the consumer, as needed.

\section{Customisation \& individualisation}

Standardised fitting systems seen in retail stores globally are not suited to assess the complexity of the versatile relationship between individual bodies and clothing. Human beings have a wide variety of body shapes and sizes and yet the current system puts every customer in discrete predetermined sizes. Current methods of developing these predetermined sizes are based on the dimension of an ideal customer, represented by a single fit model, and then other sizes are adapted through proportional increases and decreases (Raeve et al., 2012). However, one of the most intriguing alternatives to these traditional size and fitting methods is the use of a $3 \mathrm{D}$ scanner. 3D scanners are proven to be vital in identifying body size and body form (Vignali et al., 2004). According to research in The Netherlands, Germany, Spain, Britain and France, using a 3D scanner to determine clothing fit has significantly raised the share of the target group that can be dressed appropriately from 30-40\% to 50-80\% (Raeve et al., 2012).
The production of body fit parts and customisable features are not new concepts. But the task of achieving truly body fitting customisable products, using traditional skills and technology, can be quite detailed, labour intensive and time consuming (Tuck et al., 2008). The alternative is the concept of mass customisation.

Mass customisation has been defined as technologies and systems capable of delivering products that meet a customer's individual needs with nearly the same efficiency as mass production (Tseng \& Jiao, 1996). It covers a degree of customisation controlled by the consumer, where a selection of options or choices are available. Mass customisation services are mainly modular, with a production of modules that can be fastened together in varying configurations. Additionally, customisation may be in the form of color or material selection. This provides an economies-of-scale but it also allows some choices in the market for the consumer. Customisation does not offer the consumer full access to decisions about the product, the choices are limited. On the other hand, individualisation describes a deeper level of product uniqueness to the consumer. Individualisation involved products that were designed for the individual from the get go. This term better describes the design of the bra in terms of shape and size, where the bra is based off specific individual body data. Customisation may be present when the consumer decides what style or color the bra will be.

This thesis investigates the possibility of a bra system that involves 3D scanning and 3D knitting to fill the gap between the limitation of customer involvement in traditional mass customisation, and the inefficiency of a truly customisable bespoke product line (figure 3.2.). 3D scanning offers the feasibility of accurate measurements that would cater to different body shapes and in a way provides the true customisation that reflects the uniqueness of each individual. On the other hand, 3D knitting is a mass market manufacturing process, where rapid prototyping and processing is feasible. As explained in Chapter 02, Section V, with the complex 3D knitting capabilities, there is room for additional customizable features, such as specific knitting structure in certain parts of the bra for a more individualized fit. Moreover, the knitting of the bra allows for the use of the body scan measurements, which can influence the design structure of the bra. Ultimately, through customisation in the bra system, this thesis hopes to increase the consumer engagement and awareness about their own bra fit and shape. 


\section{DESIGNER}
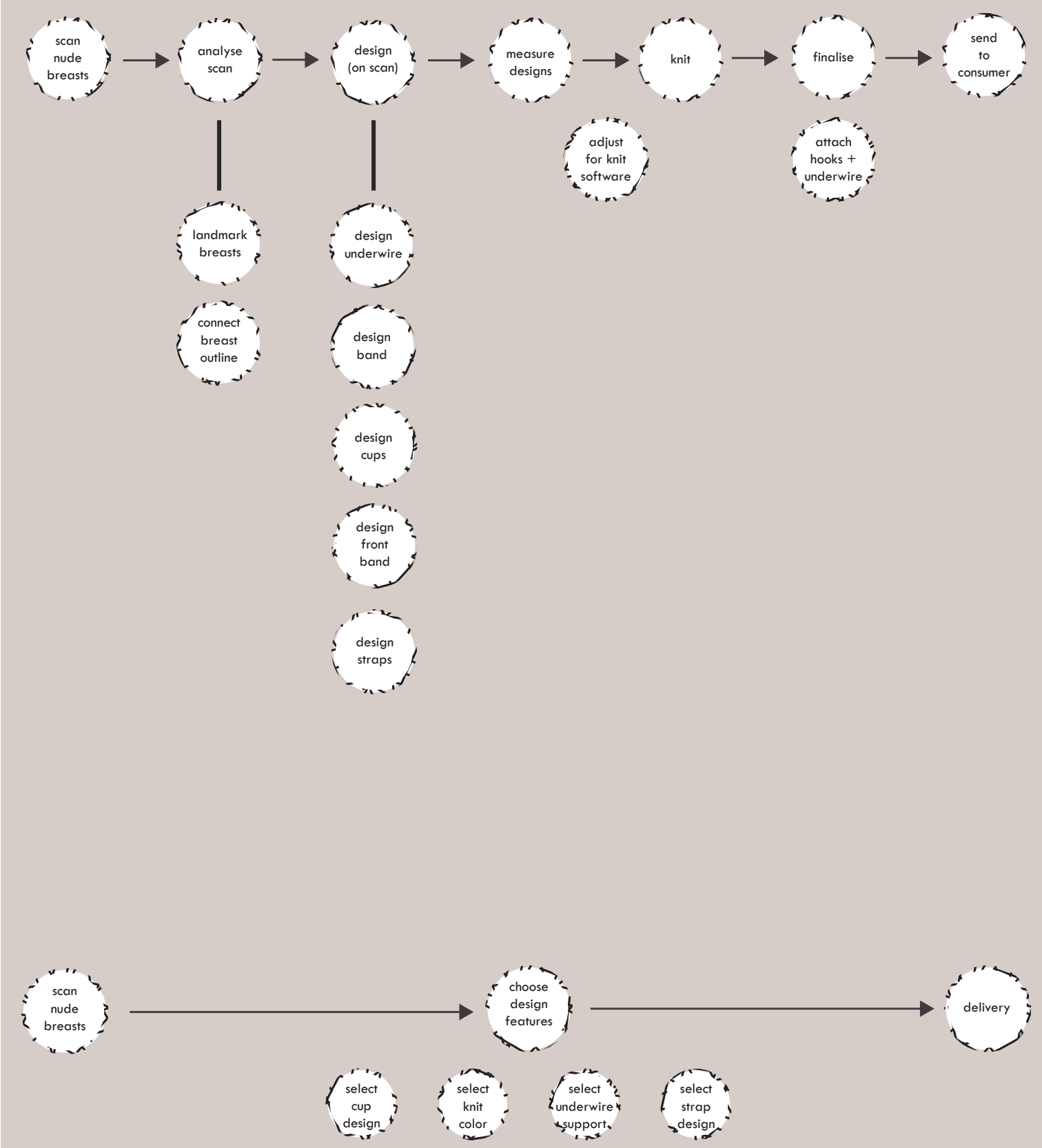


\section{DESIGN CASE STUDIES}

\section{Shima Seiki \& "Factory Boutique}

Shima"; Sandy Black \& "Knit to Fit"

The utilisation of 3D knitting technology as an approach to fashion on demand or mass customization models has been explored already. The following two case studies: the Shima Seiki "Factory Boutique Shima" retail store, and Sandy Black's "Knit to Fit" concept, presents the use of this technology to develop a system of integrating consumers as apparel co-designers, allowing for not only delivering personalized garments but also having the consumer as an integral part of the design process.

Wajima Koshan Ltd. opened a retail store, the Factory Boutique Shima, in 1997 and again in 2008 in Wakayama, Japan, specializing in on-demand production of customized knitted garments. The business concept enables a customer to enter the showroom, design and customise a flat knitted garment, and then have it manufactured promptly in a nearby production facility (Peterson et al., 2011, p. 6). The process then involves, browsing through catalogues and examining the merchandise, which are a selection of basic top and dress options; the design and customisation of the garment, where the customer is able to alter limited sections of the design, such as sleeve length; customer's measurements taken manually by a shop assistant, skilled in clothing design; pre-purchase and placing the order; manufacturing of the item; and finally the delivery of the finished garment (Peterson et al., 2011, p. 9). The Factory Boutique Shima responds to the previous gaps in customised knitted garment systems by developing a clear con-design interface between the company and the customer. The two main benefits of this system are: first, the customers are able to do a substantial amount of customisation without any assistance, and second, the customisation options are pre-programmed with the control information for the knitting machine (Peterson et al., 2011, p. 12), which cuts out time-consuming technical process of adjusting the design to be able to knit on the machine. The Factory Boutique Shima demonstrates a successful use of co-design through the employment of $3 \mathrm{D}$ knitting technology. However, this model has some limitations such as the need for a more accurate measuring system. As mentioned earlier, the measurement process is conducted manually by a shop assistant. This is not the most productive process which may be time-consuming, prone to human errors and possibly uncomfortable for certain customers. 

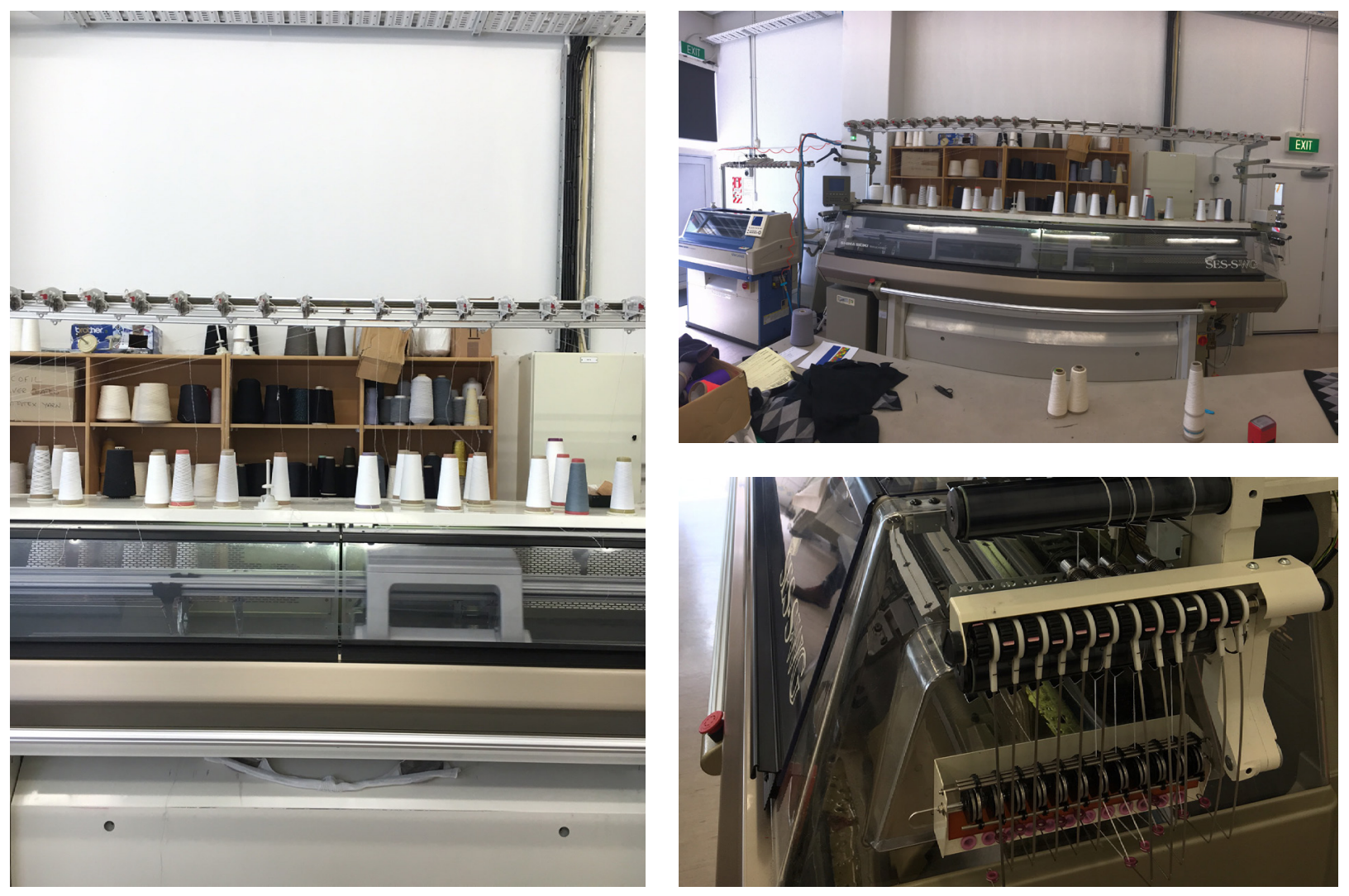

Professor Sandy Black is a member of the Fashion and Textile Design and Technology department at the London College of Fashion. In 2010, she developed the "Knit to Fit" concept as part of the research project, Considerate Design: Empowering fashion designers to think about sustainability. The "Knit to Fit" concept explores the application of 3D knitting technology and individual body measurements as means to develop personalized garments to give enhanced comfort and fit and localised individual production made to order (Black et al., 2009, p. 7). The project developed a range of seamless sweater styles customised to an individual's body shape and style preferences. This project is also addressing fit preference through "Form Fit", which allows for the application of fabric ease, where wearer to choose how tight or loose fitting their garment will be (Black et al., 2009, p. 7). The system involves three stages: $3 \mathrm{D}$ body scanning to define the measurement data set, the 'Form Fit' 2D pattern generation procedure, and the compatibility with the knitting machine to interpret the pattern profiles to produce a seamless 3D garment by means of a technical programmer (Black et al., 2009, p. 8). This project successfully illustrated the ability for 3D scanning and 3D knitting to combine as a system for individualised apparel and fitting preferences. However, this project is limited by the constraints of the 3D knitting software, where 3D measurements taken from the 3D scan must be manually translated into $2 \mathrm{D}$ for the knitting software to be programmed. 


\section{INITIAL DESIGN CRITERIA}

Based on literature search and background research, as presented in Chapter 02, we have identified a set of initial design criteria that has informed the methods and design process of this thesis. Two initial design criteria have been collated to address the bra service system proposed.

\section{B R E A S T D A T A C O L L E C T I O N}

(for the design development in Chapter 07)

Produce a clean 3D model

Identify relevant points on the breasts for bra design ( $B P, I B P, B B P, O B P, U B P$ ) (Lee \& Hong, 2006)

Scan should help highlight these landmarks (Lee et al., 2004)

Does not compress or distort the shape of the breasts (Kim et al., 2014)

Provide an alternative to other invasive methods, hands pushing upward and inward (Lee et al., 2004), or cellophane tape (Lee et al., 2004; Lee \& Hong, 2006)

Use scan data to draw comparisons between participants of the same bra size (selfdetermined bra size 12D)
Only a clean 3D model without extra scan meshes/data will allow for accurate measurements

Use these points to draw out an accurate breast outline which will be used for design development of the underwire line, as well as cup to band connection

This will skew breast measurements

These other methods are unreliable due to inconsistencies with how they may change or distort the breast shape

This will help validate the complexity of the breast shape, and help highlight differences between breast shapes of participants who share the same bra size. 


\title{
B R A D E S I G N D E V E L O P M E N T: 3 D K N I T T I N G E X P L O RA T I O N
}

\author{
(for the design development in Chapter 08)
}

\section{Criteria}

Design is to be based off measurements gathered from the 3D scan, specifically using the landmarks from literature

Asymmetry and differences in measurements
between left and right breasts are accounted for
Measurements take into account movement and
material stretch

Designed within the limitations of the 3D knitter

Fabric is breathable and flexible
Insure that the the design of the bra corresponds to actual individual body data which will provide a good fit

The bra structure should reflect the individual body data because no two breasts are exactly the same

Yarn and fabric choice will alter measurements, so body data measurements must be reviewed at the knitting stage

Underwire is consistent with actual breast outline and underline

Breast outline is one of the most important measurements for the bra design, an accurate underwire that fully supports and shapes the breast can only do so if it accurately reflects the breast outline

This will help validate the complexity of the breast shape, and help highlight differences between breast shapes of participants who share the same bra size.

Material is soft and non-restricting to the skin

Uses unique properties of the 3D knitter, in other words, uses specified knit structures

Bra design acknowledges fit criteria
For example, knit structures should be used thoughtfully to help with pressure, stretch and fabric density

Refer back to the fit criteria (fig. 2.7) to check that the design itself is inherent to a good fit 

C H A P TER 04

METHODOLOGY 
ঠे 
The purpose of this chapter is to present the methodology needed for the design phase. The last chapters reflected upon the background research, including literature reviews to assess the limitations and successes of previous work on bra purchasing behavior, bra fit and sizing, breast measurement and bra design processes. This background knowledge has helped inform a set of initial criteria (as noted in Chapter 03) to influence the following design phases and verify the methods and technology choices. This chapter will discuss these methods and where they will be applied in the four phases of design. The first four design phases, Chapter 05, Understanding the Bra Experience, Chapter 07, Breast Data Collection, Chapter 08, Bra Design Development: 3D Knitting Exploration, and Chapter 09, Bra Design Development, will directly influence the final output, Chapter 10, Results.

This chapter addresses the human factor in bra buying, fitting and design. The bra system should not operate independently of its intended users and must always connect back to individual needs at every stage.

Therefore, this research could also be considered part of a human-centered design methodology. As such, this research will be conducted as a mixed-methods approach (Creswell \& Plano Clark, 2011) with a combination of qualitative and quantitative processes. A rich qualitative data set is valuable in a topic that evokes a very personal and emotional response (Freeman, 2008). However, without grounding it in, or including support from, quantitative analysis, there will be only limited context for validating the findings.

The bra design and physical output (e.g. the actual bra) will be based on an iterative and exploratory prototyping process to which a research through design methodology has been adopted. Research through design (Martin \& Hanington, 2012, p. 146) recognizes the design process as a research activity. Research through design is distinguished by the design work itself, through, for example, material research, critically recording and analysing steps of the design process, experiments and iterations. 


\section{Interviews}

Informal, semi-structured interviews (Martin \& Hanington, 2012, p. 102) were conducted with each of the four participants to contextualize an understanding about their personal bra fitting and buying experiences. The interviews were open-ended questions and were aimed to lead the participants to discuss any positive or negative feeling or attitudes towards the bra experiences. A further two interview sessions were conducted with one the participants during user-testing the bra prototype. The first interview session was to receive feedback on the first bra prototype, and the second interview was to receive feedback on the altered bra prototype. A copy of the interview questions can be found in Appendix III.

\section{Content analysis}

To systematically examine the information gathered from the semi-structured interviews. The author conducted a content analysis (Cole, 1988), specifically an inductive content analysis to categorize and code the data. Content analysis is a research method for making valid inferences from written, verbal, or visual communication methods to their context, with the purpose of providing new insights or a representation of facts (Krippendorff, 1980). In this research, a collection of similar word phrases and key terms were drawn from the interviews and established into categories or themes. These categories helped establish a more quantitative output to the qualitative information set. Through a content analysis the author could notice what overlapping preferences and attitudes towards bras were consistent between the participants.

\section{Questionnaires}

The participants completed a short questionnaire, which aimed to establish an understanding of each participant's basic bra and breast knowledge, as well as participant's rating for their own bras. The first part of the questionnaire included short answers and multiple-choice answers. In the second part, the participants were asked to rate five of their own personal bras based on categories of comfort, support, aesthetic, style, shape worn with clothes, underwire function, and how often worn. A copy of the questionnaire can be found in Appendix III. 


\section{D Scanning}

3D scanning is used as the primary measuring technique to obtain the breast data needed for this thesis. 3D scanning is a tool that is not limited to acquiring one type of measurement. As discussed in Chapter 02, Section III and V, 3D scanning has been validated as accurate for obtaining breast shape in three dimensions, as well as a good tool for breast imaging for acquiring breast measurements.

Unlike other methods, 3D scanning allows for a collection of large quantity of measuring and imaging data with a single scan. In this thesis, three scans of each of the participants was conducted in a private space. The Artec Eva 3D scanner, a hand-held laser scanner that creates a 3D image was used for all the scans. Multiple flash bulbs are projected onto the participant from the hand-held device and a sensor measures the distance to the surface (Ebrahim, 2011). The scanner is attached to a computer with software that allows visualisation and manipulation of the 3D images captured. The Artec Eva has the ability to make quick, textured and accurate imaging, with a $3 \mathrm{D}$ resolution up to $0.5 \mathrm{~mm}$.

\section{Parametric modeling / Rhino Grasshopper}

The parametric CAD software, Rhino/Grasshopper, was used to extract the body data and appropriate measurements obtained from the 3D scanner. Through this software, the $3 \mathrm{D}$ model is manipulated and analysed to assess accurate breast measurements needed specifically for the bra design process. Grasshopper is a parametricbased software within Rhino 3D system that allows the user to apply the same measuring processes to each $3 \mathrm{D}$ scanned model that is inputted into the program, instead of measuring manually each time. The software stores the designed formula needed to extract the measurements.

\section{D printing}

$3 \mathrm{D}$ printing is the primary method for achieving underwire designs unique to the participant. The designs will be drawn parametrically in Rhino/Grasshopper and then printed using 3D printing. This method is used for rapid prototyping as well as achieving detailed complex curves that are based off actual body data. 3D printing techniques allow for individual customised parts to be printed rather than needing expensive tools for producing the same part. The Objet Connex 350 is capable of blending different material types at various ratios. This printer offers the ability to print flexible, rubber-like properties as well as stiff ones all in the same object. It was these properties that was advantageous to explore the possibility of a dual textured underwire prototype.

\section{D knitting}

$3 \mathrm{D}$ knitting is used to produce the seamless design of the bra base, cups and straps. As explained in Chapter 2, 3D knitting has advantageous properties such as creating seamless features within one panel. It also allows for creating uniquely sized tubes to be slotted into the underwires. Additionally, 3D knitting allows for experimenting with various knitting structures and a combination of yarn types. This will create new fabric types unique to the bra design, as well as deliver specific pressures and stretches in different parts of the bra, where necessary. The Shima Seiki, flat-bed SES-S "whole garment" is the 3D knitting machine of choice. Most New Zealand companies have invested in Shima Seiki knitting machines and therefore there is a support system in place for this research (Smith, 2013). The 3D knitting machine is usually only seen in a commercial context. However, the Textile and Design Lab at Auckland University of Technology (AUT) has acquired three Shima Seiki machines to be used for the purpose of research and design. The 3D knitting process will be the primary design method for achieving the final designed output. The knitting was conducted through an iterative process where each prototype helps influence the next.

\section{Iterative process}

An iterative process is a process of refinement (Karjaluoto, 2013, p. 150), where each iteration is analysed and making subsequent adjustments until the goal is reached. The iterative perspective was used to develop knitted bra prototypes which encompassed the desired shape, form, materials and knit structures. Following the iterative process methodology, each step in the progression was documented and discussed so that an informed decision could be made of how to improve the design for the following.

\section{User-testing}

Testing can be an important part to the design process. Testing, can offer on-going validation that can help determine whether the design is suitable or needs more attention (Karjaluoto, 2013, p. 159). User-testing was conducted as part of the design process in this research. First to analyse a set of initial knitted prototypes and gather participant feedback, then, after implementing this feedback, a second round of testing was conducted to deliberate the resulting design. 
A small pool of four volunteers, women ages 18 to 25 , with the self-identified bra size of $12 \mathrm{D}$, were called to participate in this research. The research needed to follow methods for an in-depth understanding of their personal bra experience, which called for a small number of volunteers to work in depth with them. Cup size D was selected because as discussed in earlier chapter, the average cup size has risen from B to D in the last years, and sizing charts, design process and fitting methods have not accounted for this change. The study also asked for participants to self report their bra size (12D selected for the study) to compare participants' reporting with their actual breast shape, and determine fit variations among all participants who are considered the same size. Lastly, the research selected participants with ages of 18 to 25 , because the youth population has a higher probability of not to seeking fitting advice and are more likely to be open to new technologies (Tsarenko \& Strizhakova, 2015, p. 45). 


\section{MET HODS A P P L I E D}

The design process for this thesis has been broken down into four distinct phases. The table below offers a brief description of the each design phase and the methods applied.

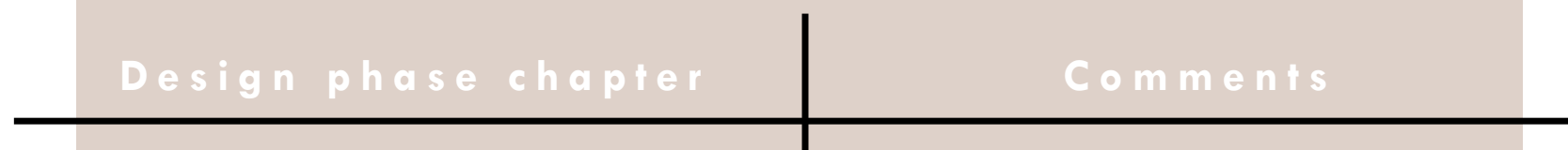

Chapter 05

Understanding the bra experience

Collection and assessment of a set of narratives based on participant's bra fitting and buying experience, basic knowledge of bras and breasts, as well as getting insight to the participant's bra fit and style preferences.

Methods: Interviews; questionnaires; content analysis

Chapter 07

Breast Data Collection
Acquisition of key data points on breasts shape and measurements to better understand breast topology

Methods: 3D scanning; parametric modelling

\section{Chapter 08}

Bra Design Development: 3D Knitting Exploration
This phase involves an exploration of non-traditional technologies and processes applied to bra design development. The iterative prototypes and experiments in this stage ultimately can lead to the final design, presented in the final phase.

Methods: 3D printing; 3D knitting; iterative process; user-testing

\section{Chapter 09}

Bra Design Development: Utilizing Measurements
Final bra designs are prototyped based on data from phases 1-4. Two final outputs that showcase the past experiments were developed and analyzed.

Methods: parametric modelling; 3D printing; 3D knitting; user-testing 

C H A P TER 05

BRA EXPERIENCE 
In order to design, develop and manufacture a wellfitted bra, all aspects of the bra must be understood and integrated, and they must be done on a human level, where the wearer is a central part of the overall system. In other words, reliance on literature or industry standards alone is not sufficient, and bra wearers participation and input are essential part of the ingredient for a good fit.

This chapter presents bra wearer's experience in terms of bra buying, fitting and design preferences. Through semi-structured interviews and brief questionnaires, the participants of this research project were able to narrate their own bra story. The interview questions were open ended and provided an introduction to the participant's personal bra experiences, preferences and suggestions. Additionally, the written questionnaire offered a brief insight into each participant's bra and breast knowledge. At the end of the questionnaire, participants were given the opportunity to assess five of their personal bras. The first four figures of this chapter (figures 5.1. to 5.4.) display the interview and questionnaire data in a collated manner. Thereafter, the data is demostrated through the individual participant responces (figures 5.5. to 5.12.). 


\section{"Current bras create this 'idealised', 'boost-up', 'breasts on display", look. It becomes about you thinking you are wearing a bra for other people instead of for comfort and the shape you already have. Bras should portray a realistic representation of different breast shapes and sizes."}

- Participant No. 3

\section{BRA SIZE}

Throughout the interviews and questionnaires the subject of bra sizes where discussed. The question of what bra sizes the participant considered herself was asked in the questionnaire, and bra sizes were again mentioned when the participant was rating her own five bras. In the end, each participant noted that she was more than one bra size. The size depended on bra brand, style, or whe ther they were old bras or new ones with a new size. The figure to the left simply demostrates the range of bra sizes for each participant. Though the participants called for this study were to be a $12 \mathrm{D}$, none of the participants were soley a $12 \mathrm{D}$. This suggests the variety of sizing for each body shape. Though the common bra size is $12 \mathrm{D}$, each participant also had a unique bra size that none of the other participants shared. 


\section{PA R T I C I PA N T B R A S I Z E S}

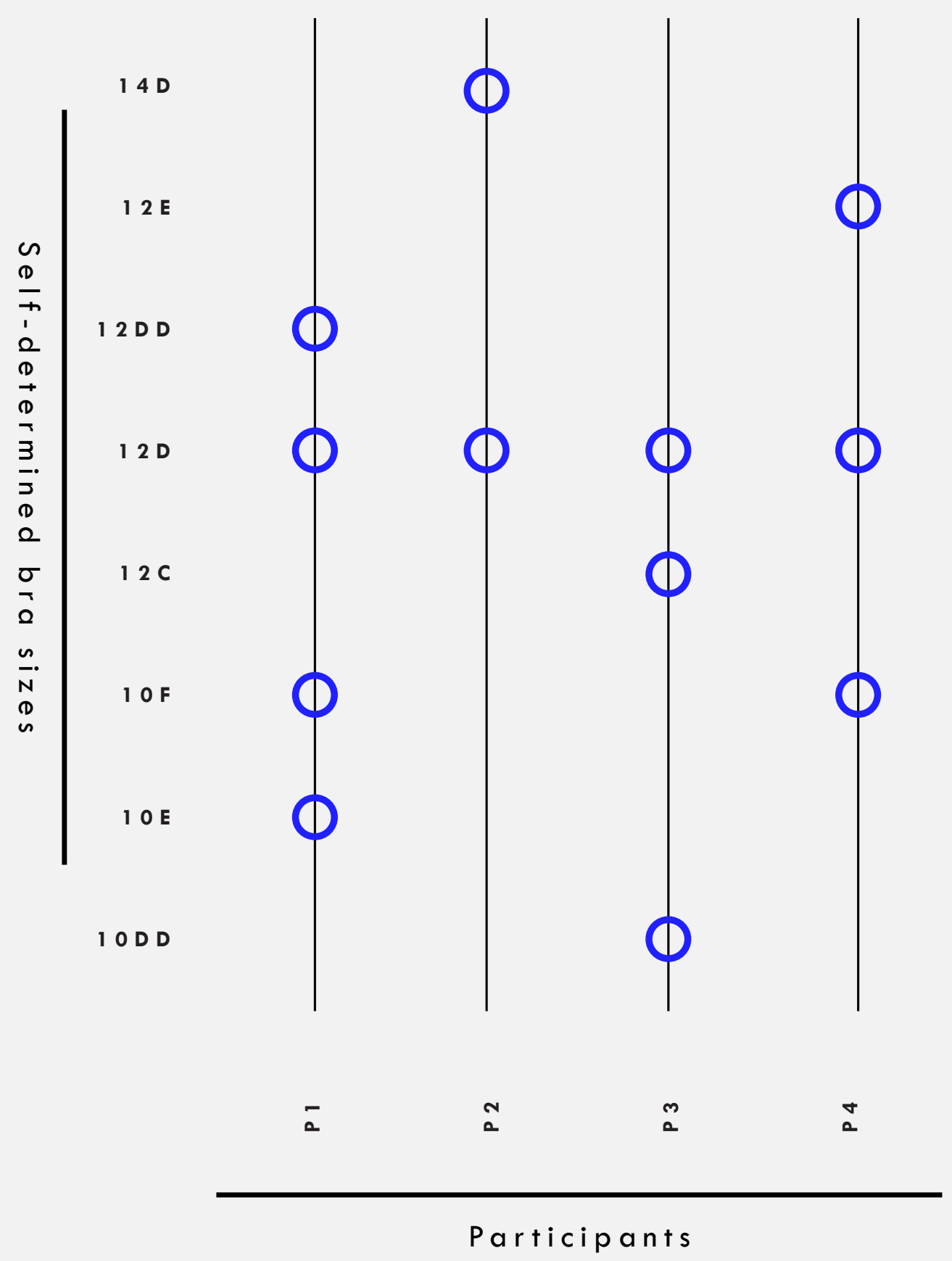




\section{P A R T I C I P A N T I N T E R V I E W S}

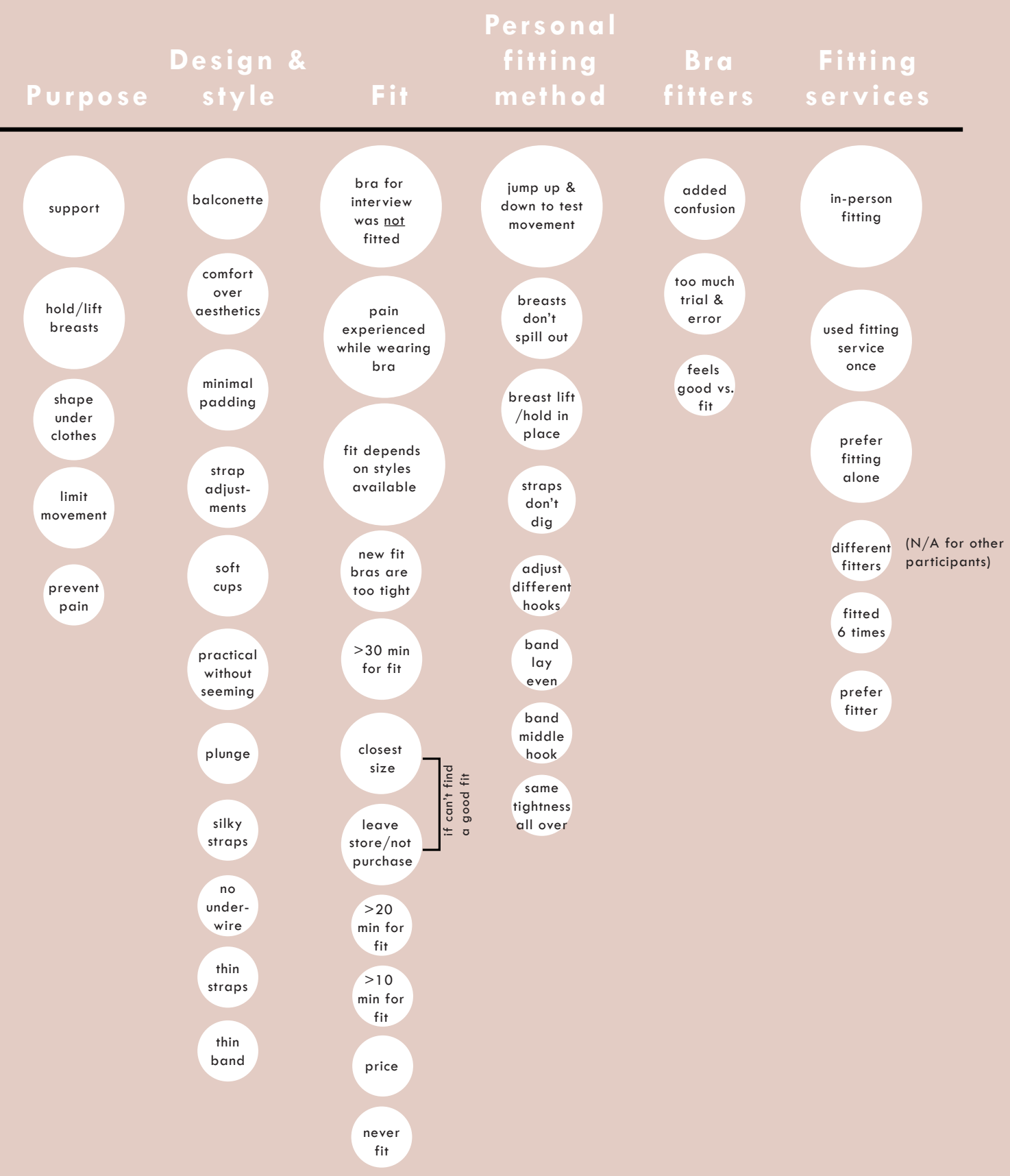

Participant key words \& phrases corresponding to each theme

Ordered from most amount mentioned to least 


\section{INTERVIEWS}

The aim of the interview was to collect narratives of bra fitting and bra buying experiences for women around the same age and breast size. These narratives provided guidance on individual bra attitudes and perceptions. The author met with the participants individually in a relaxed private environment. The interview followed a series of open-ended questions of which the participant could answer as long or as short as they desired. The interviews were analysed by collating the participant responses into six overall themes: purpose of wearing a bra; design and style preference; fit; individual fitting method techniques; thoughts on bra fitters; and fitting service experiences. The following graphics illustrate these six categories, followed by recurring key terms and phrases used by each of the participant's responses. The key terms and phrases were placed in each section accordingly. The collated result of the interviews follows the same system of six categories followed by key terms and phrases. However, the key terms and phrases used in the collated result are organized by hierarchy, where the size of the circle indicates relative number of participants sharing the same phrases or ideas. 
The questionnaires were conducted in two sessions. The first part involved short answers and a multiple-choice format in response to the participant's personal bra habits, as well as basic breast and bra knowledge. The second part asked participants bring five of their bras, which they rated on a Likert Scale ( 5 for most valued and 1 being least valued), based on comfort, support, aesthetic, style, shape worn with clothes, underwire function, and how often worn. Data collected from the participant's personal bras were described by type of bra (moulded, non-padded or padded), style of bra (balconette, plunge, sports, long-line, or soft-cup/bralet), and color (black, blue, nude, red, pink, or green). The data displayed individual bra ratings for each participant as well a collated version to compare bra rating based on the type of bra and style of bra.

\section{P A R T I C I P A N T B R A R A T I N G}

Collated data of participant bra ratings

based on bra type

least valued to most valued

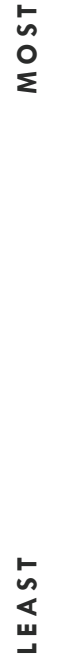

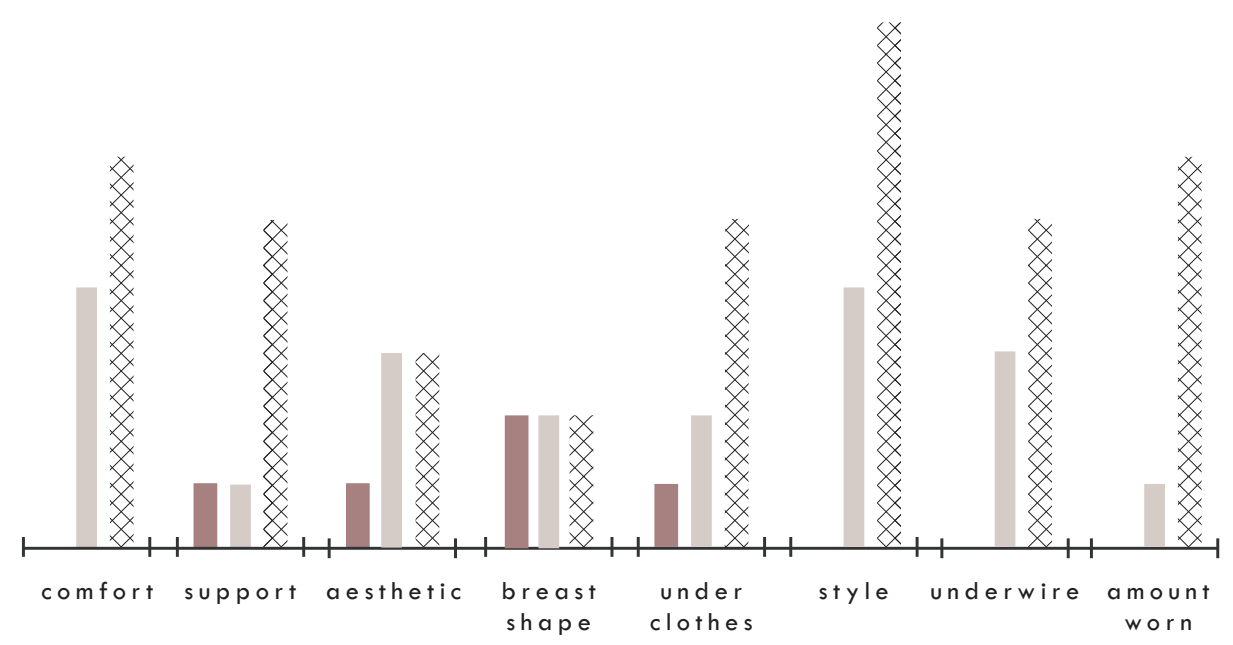

PA D D E D

M O ULDED

NON-PADDED 


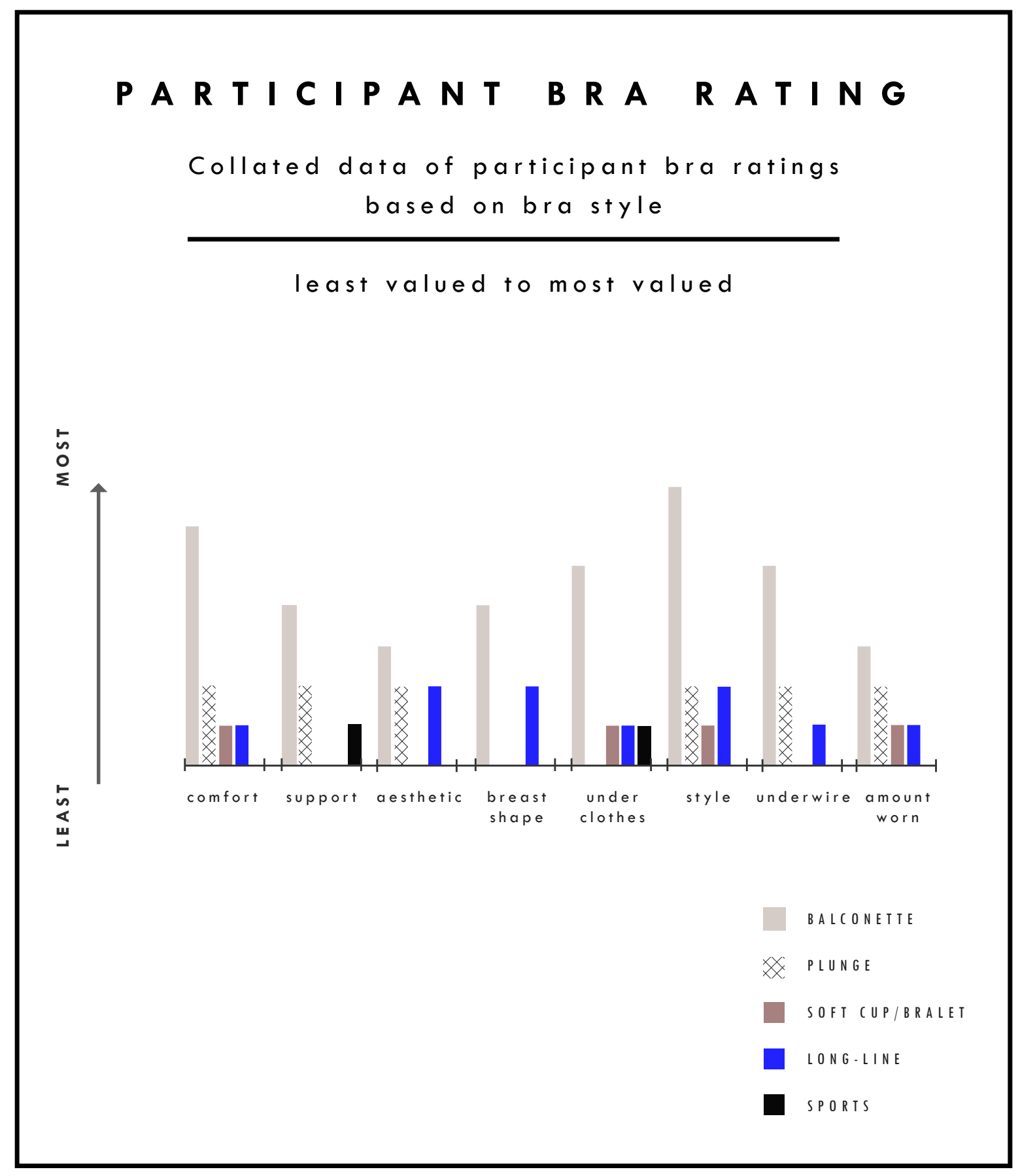


PARTICIPANT

$\mathbf{N}$

O. 1

$\stackrel{\infty}{\circ}$ 
"I hate trying to find the right fit, because you don't always want to ask the lady but if you just go for the same size there's a lot of Variability in what you get. Also I'm pretty active during the day and a couple of bras rub like they're not contoured properly to the body shape or sometimes the wire works its way out both resulting in un-comfy or rew skin." 
PARTICIPANT NO. 1

\section{N T E R V I E W}
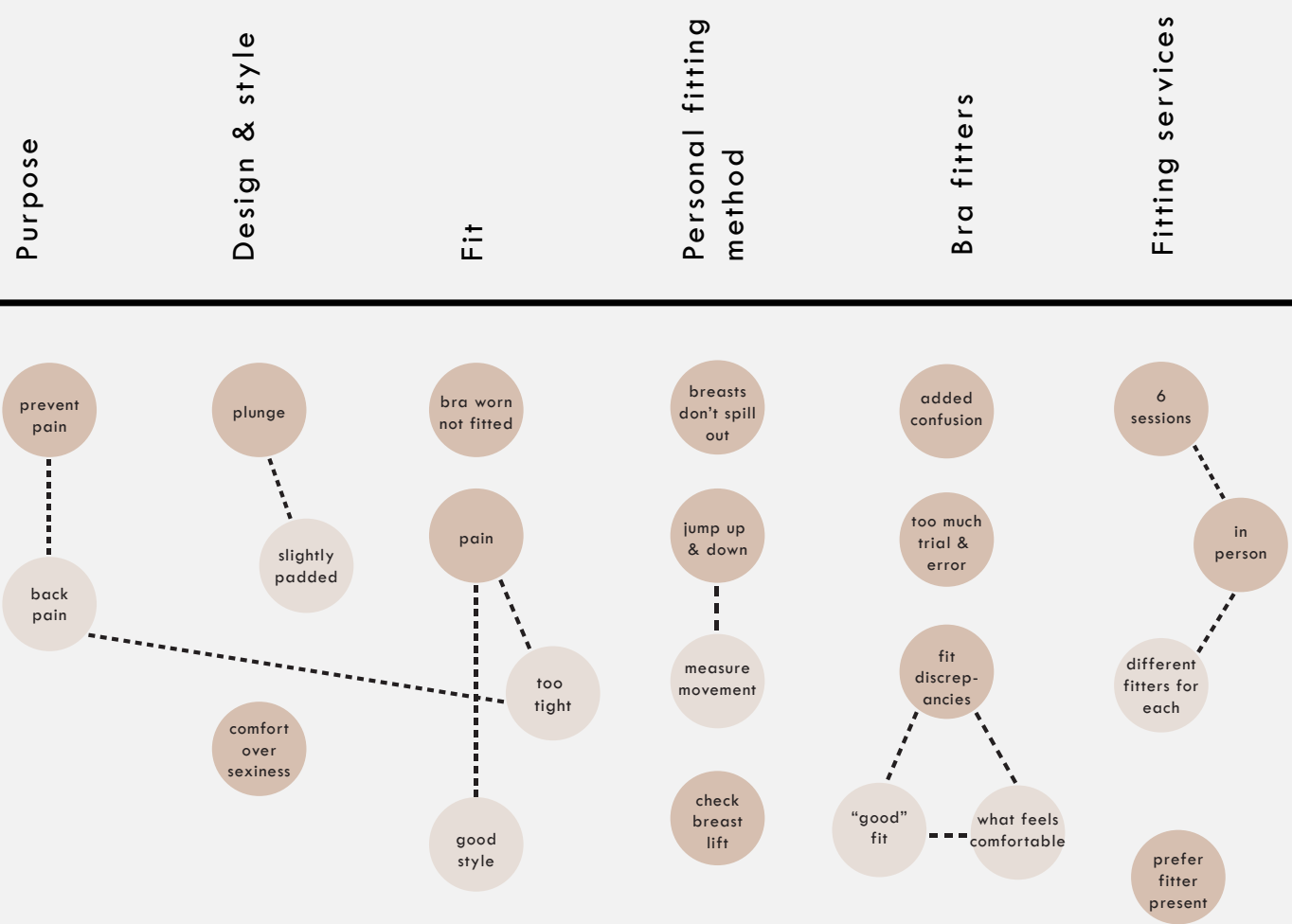

bad
style

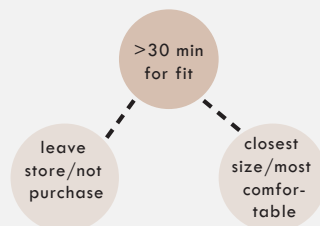


PARTICIPANT NO. 1

\section{$\begin{array}{lllllllll}B & R & A & R & A & T & \text { I } & \mathbf{N} & \mathbf{G}\end{array}$}

Participant No. 1's bra ratings

based on personal bra selection

least valued to most valued

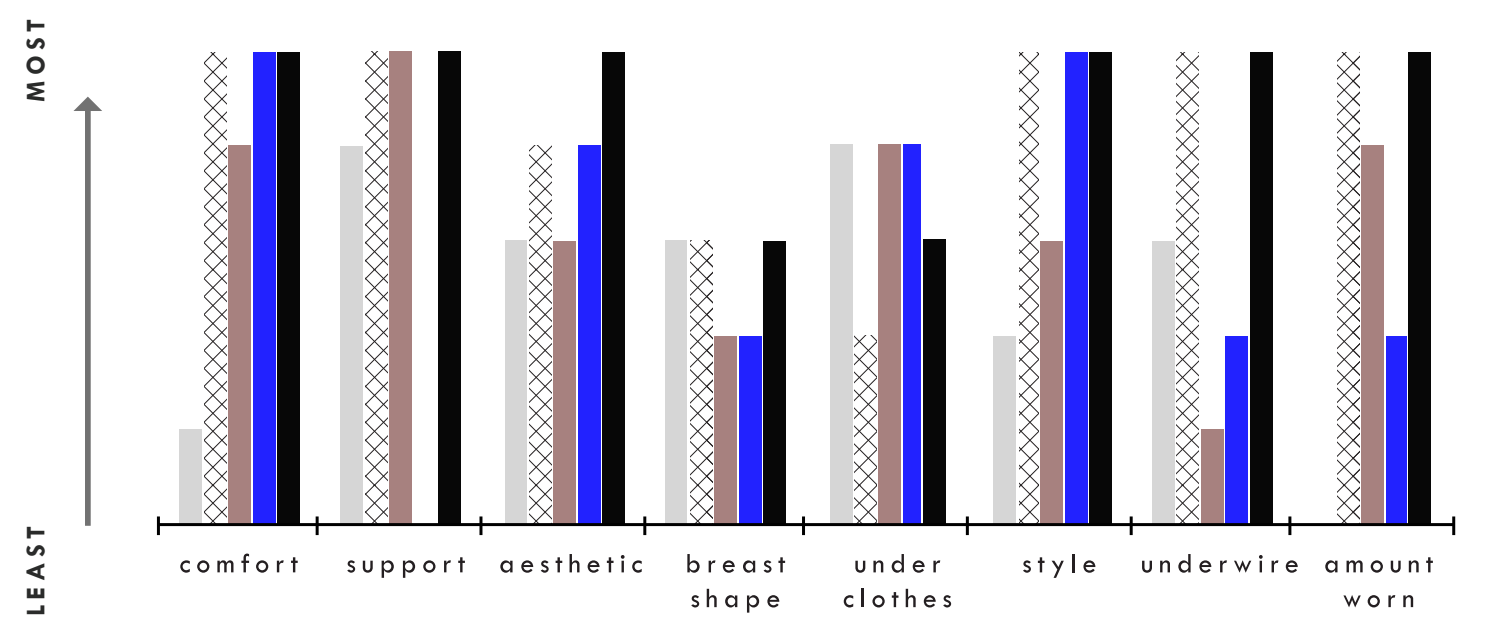

PADDED / BALCONETTE / BLACK

Non-PAdDED/PLUNGE / BLUE

NON-PADDED / SPORTS / BLACK

Moulded / balconette / black

WON-PADded / PLUNGE / BLACK

Five of Participant No. 1's personal bras

were used for this exersize. Each bra

was distinguished by bra type,

bra style, and color. 
PARTI CIPANT

$\mathbf{N}$<smiles>O</smiles>

2

○ 
"I like thinly padded bras, or lace $\mathbf{S}$ oft-cup bras with underwire. I like them the best and wear them the most. Sometimes cups are too close together for me, and sometimes the cups are too big when I try and find the right fit around the back - because I'm quite brodd." 
PARTICIPANT NO. 2

\section{N T E R V I E W}

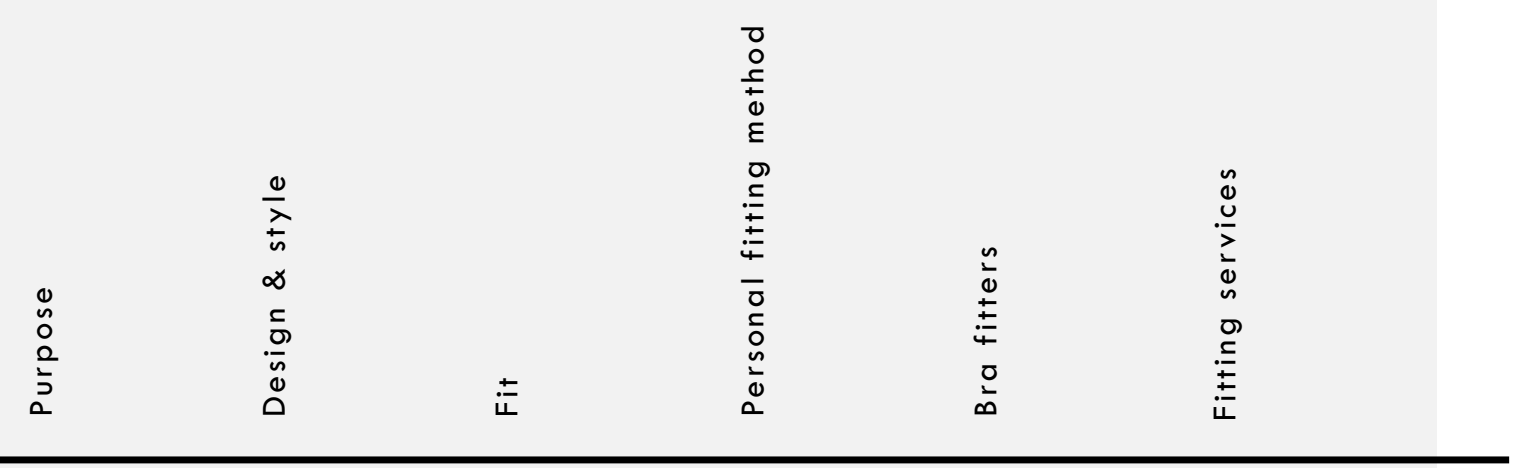

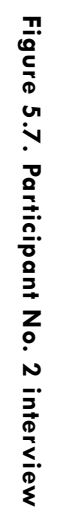
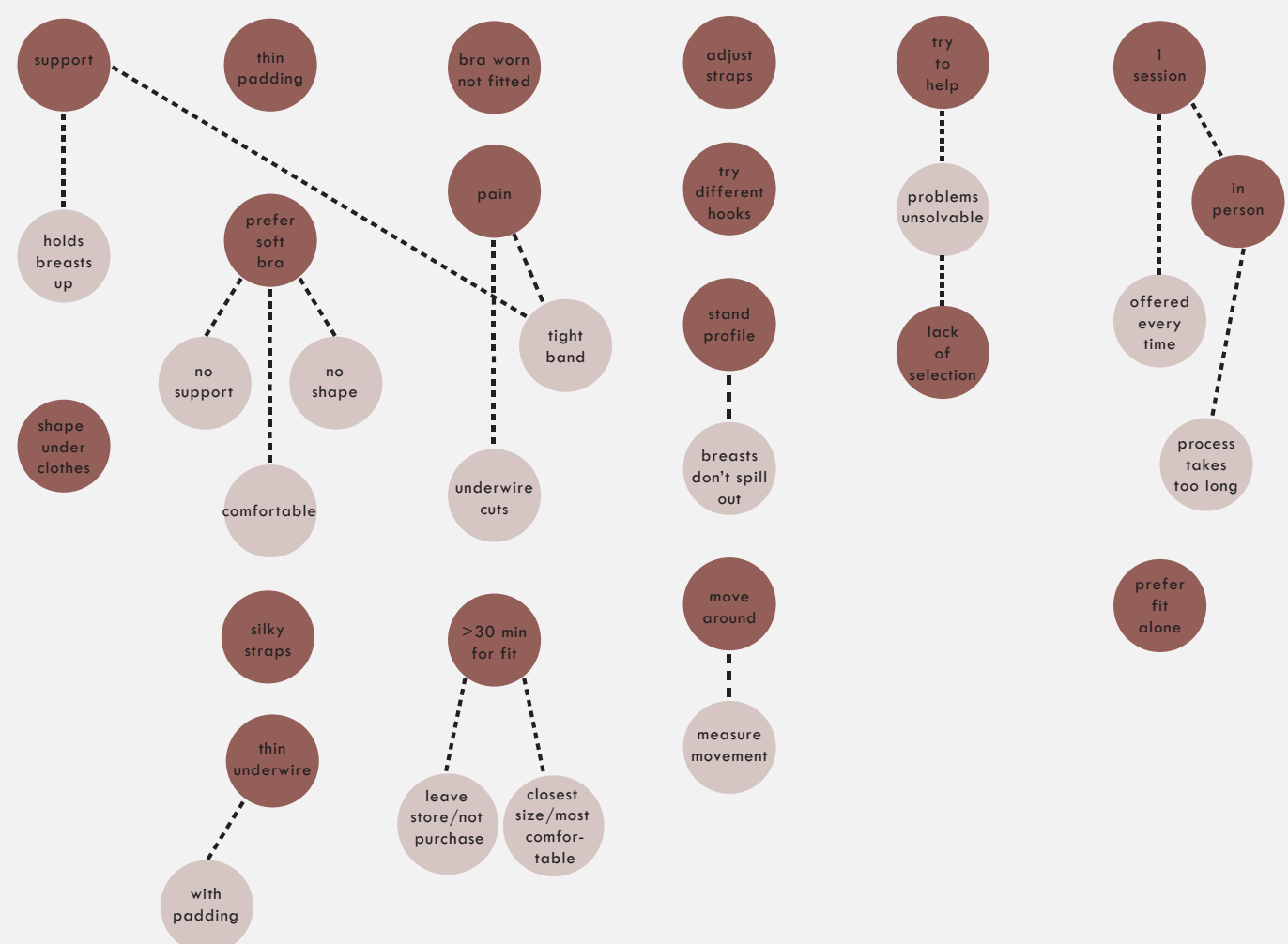

$\stackrel{\circ}{\sim}$ 
PARTICIPANT NO. 2

\section{$\begin{array}{lllllllll}B & R & A & R & A & T & \text { I } & \mathbf{N} & \mathbf{G}\end{array}$}

Participant No. 2's bra ratings

based on personal bra selection

least valued to most valued
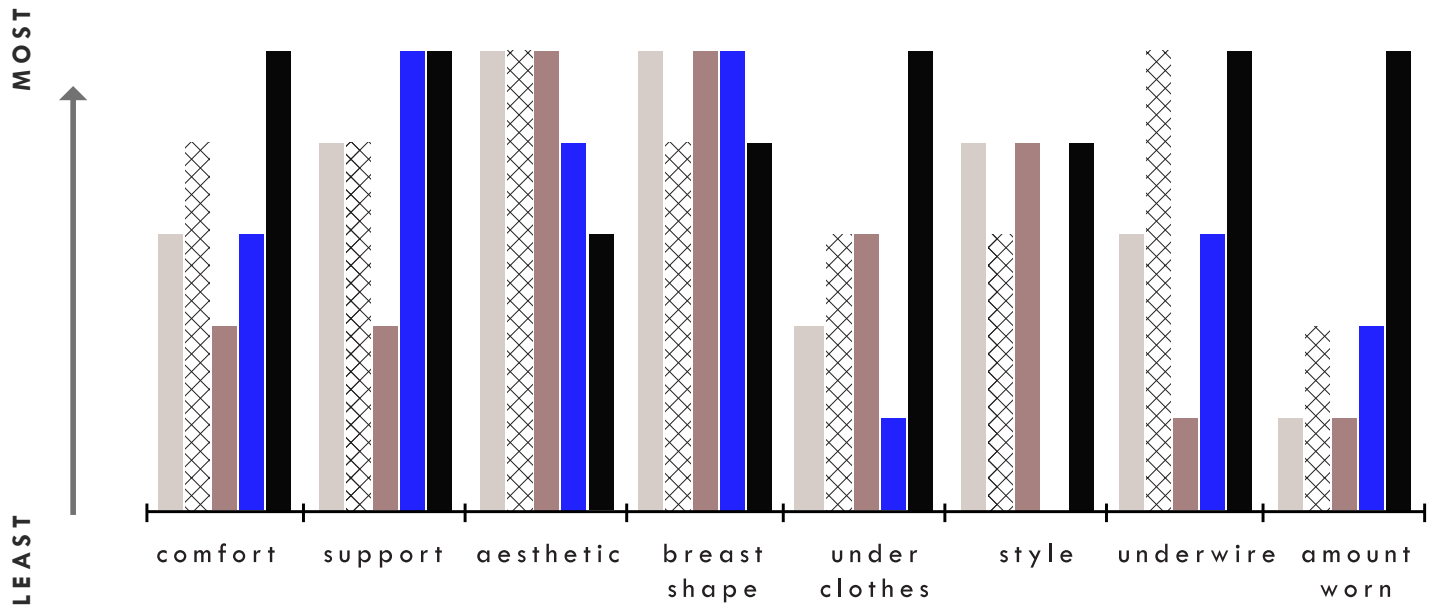

MOULDED / BALCONETTE / BLUE

X MOULDE / BalCONETte / RED

NON-PADDED/ LONGLINE / BLACK

PADDED / BALCONETTE / PINK

NON-PADDED/ BALCONETTE / GREEN

Five of Participant No. 2's personal bras were used for this exersize. Each bra

was distinguished by bra type,

bra style, and color. 
PARTICIPANT

NO. 3

$\stackrel{\circ}{\perp}$ 
"l like soft-cups with no

underwire, no padding

either. ldeol bra would be

n o bre. So something

that holds in place but you

can borely notice

would be perfect. Problems

arise when there is on

uncomfortable underwire,

which is restricting, and

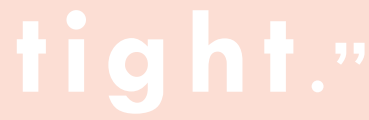


PARTICIPANT NO. 3

\section{N T E R V I E W}

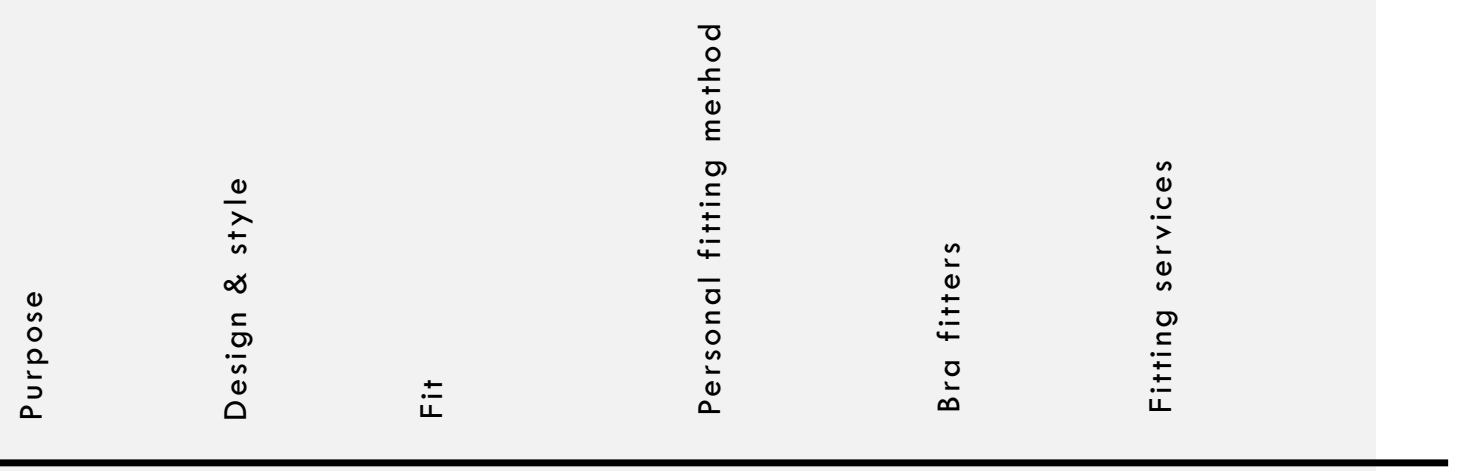

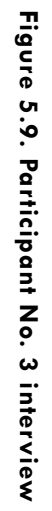
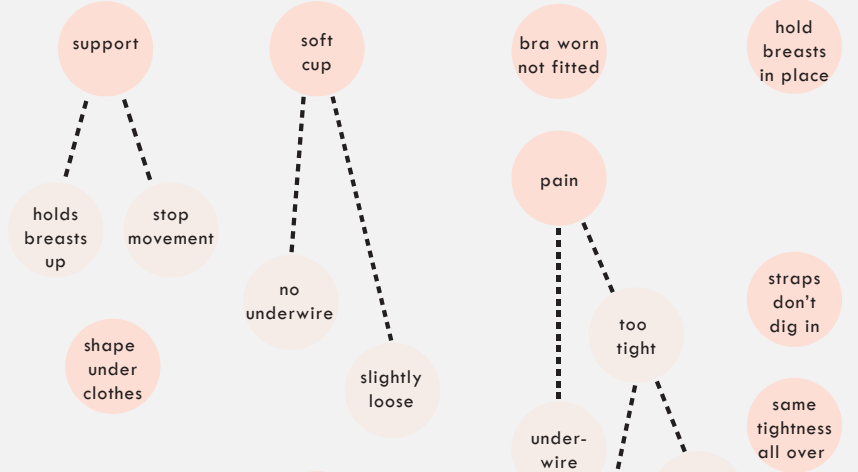

added
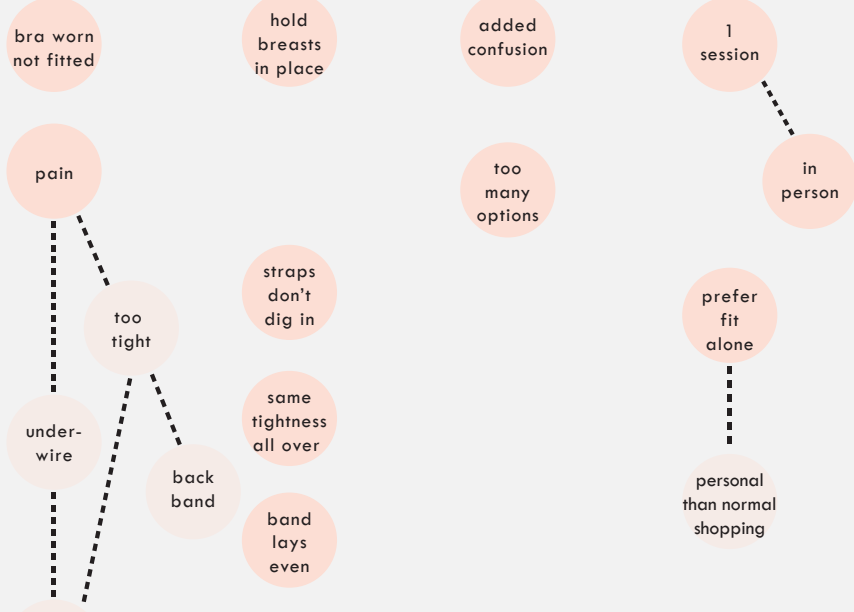

too

many

person

feel like

wearing
no bra

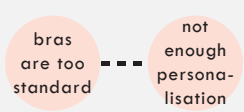

under
bust

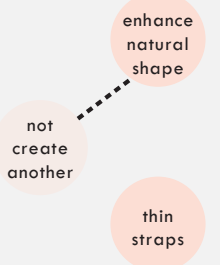

$\begin{array}{cc}\text { may fit - - - } & \text { not at } \\ \text { home }\end{array}$

instore

home

$a$ 
PARTICIPANT NO. 3

\section{$\begin{array}{lllllllll}B & R & A & R & A & T & \text { I } & \text { N } & \text { G }\end{array}$}

Participant No. 3's bra ratings

based on personal bra selection

least valued to most valued
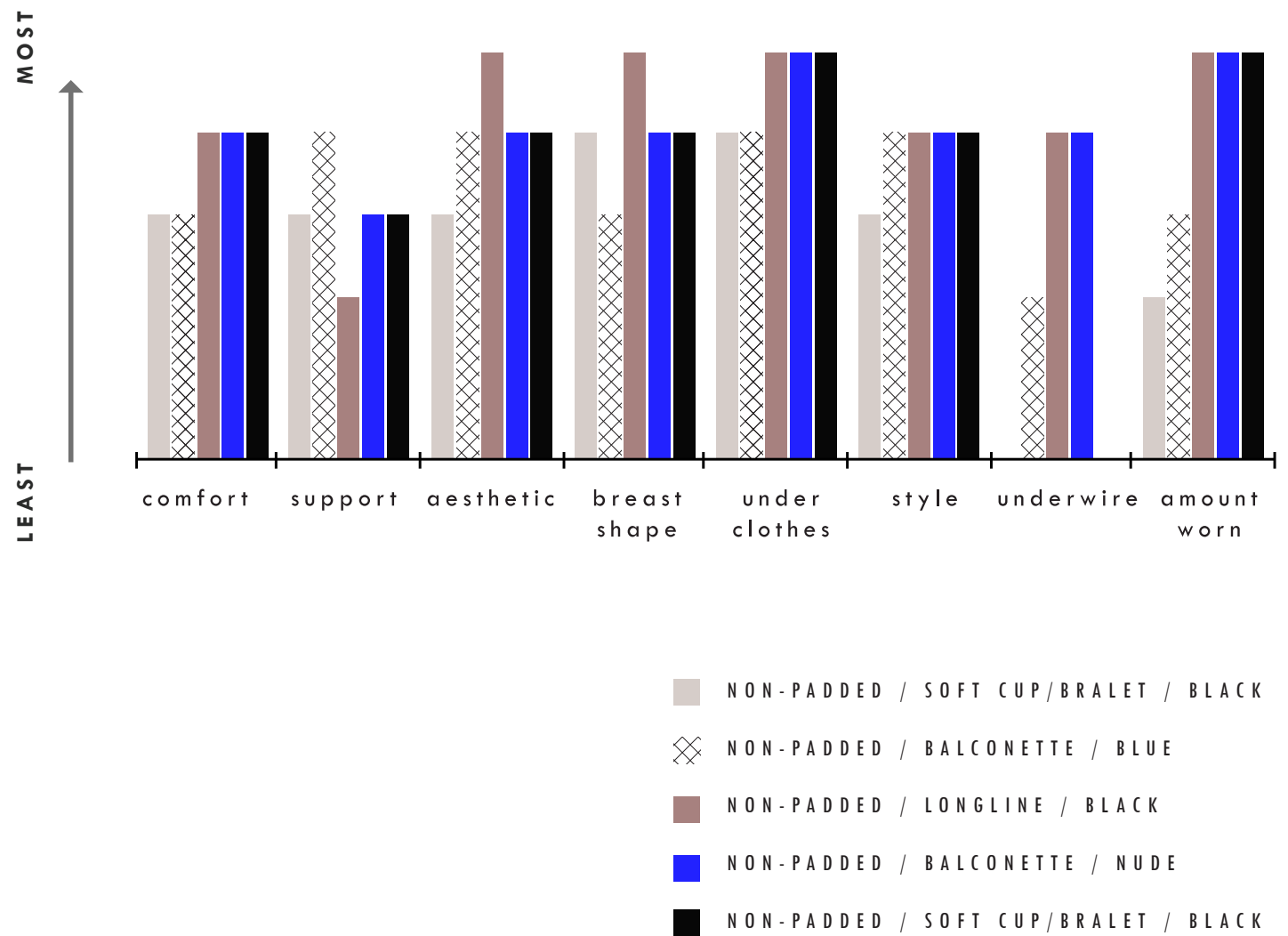

Five of Participant No. 3's personal bras

were used for this exersize. Each bra

was distinguished by bra type,

bra style, and color. 
PARTICIPANT

$\mathbf{N}$

O. 4

$\infty$ 
"I mostly wear balconette bras. Cotta strap em in! However, there

are no nice bras in bigger sizes, shoulder straps can tend to hurt after an active day." 
PARTICIPANT NO. 4

\section{N T E R V I E W}

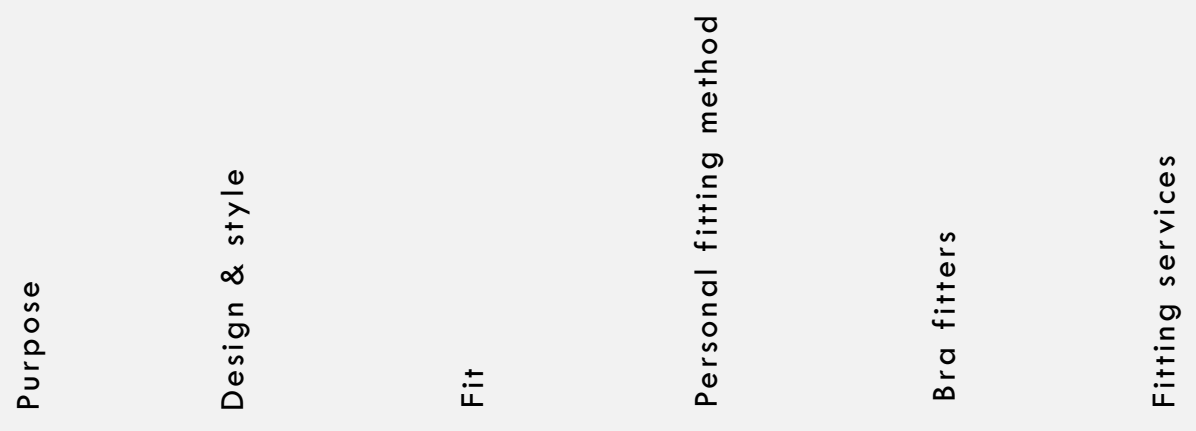

7
0
$c$
0
0
0
0
0
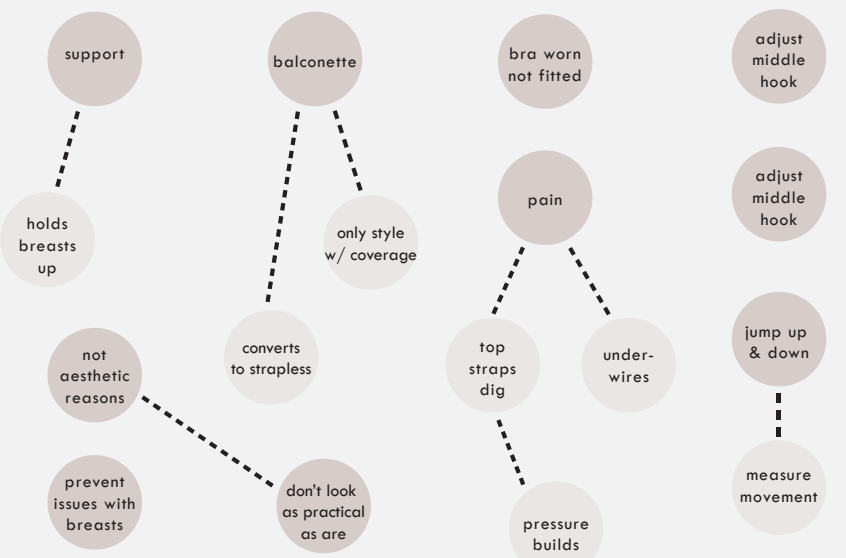

\section{helped
find a fit}

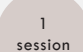
in

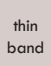

thin
band

thin
straps

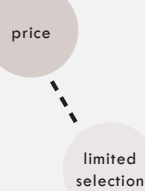

plunge

w/o spilling

selection

breasts

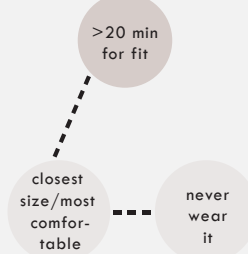

$\vec{\circ}$ 
PARTICIPANT NO. 4

\section{$\begin{array}{lllllllll}B & R & A & R & A & T & \text { I } & \mathbf{N} & \mathbf{G}\end{array}$}

Participant No. 4's bra ratings

based on personal bra selection

least valued to most valued

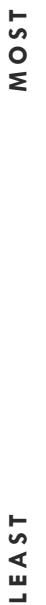

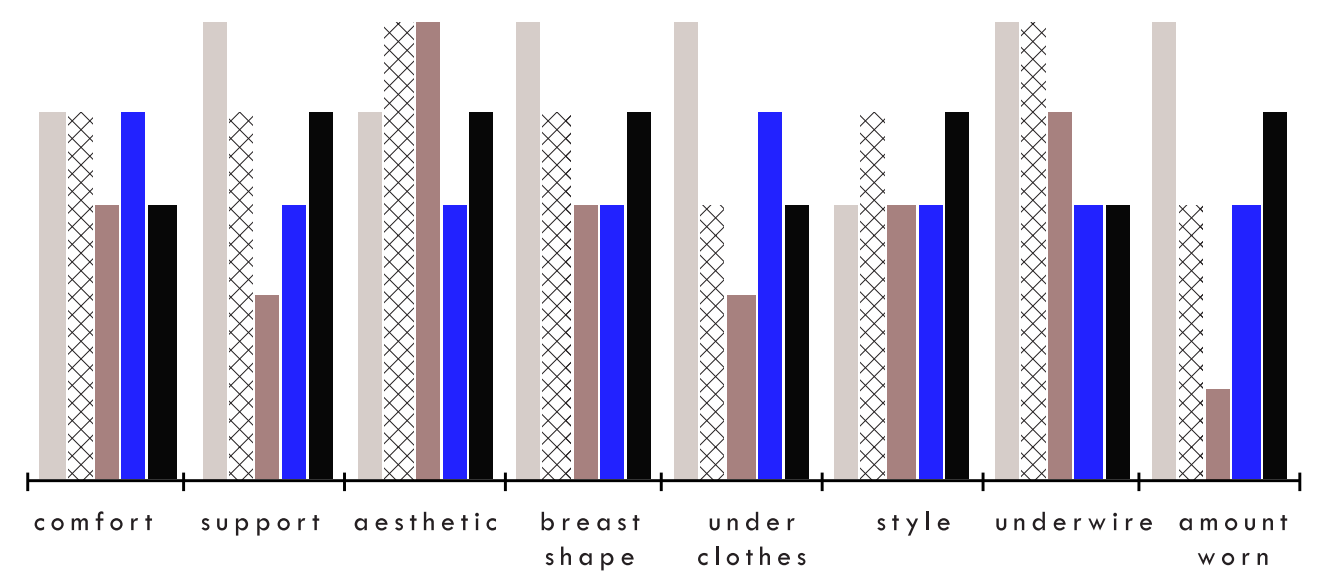

MOULDED / BALCONETTE / BLACK

X MOULDED/BALCONETTE / RED

PADDED / PLUNGE / PINK

MOULDED / BALCONETTE / PURPLE

MOULDED / BALCONETTE / WHITE

Five of Participant No. 4's personal bras

were used for this exersize. Each bra

was distinguished by bra type,

bra style, and color. 
CHAPTER 05

BRA EXPERIENCE

DISCUSSION 
The most common theme emerged during discussion of bra fit with participants: occurrence of discomfort and pain at some point while wearing a bra, and high dependency of good bra fit on the styles offered at the store. Additionally, though all participants had experienced being fitted by a professional fitter at a retail store, none of the bras worn at the time of the interview had been fitted. Only one participant had been fitted this way more than once and preferred a fitter to be present while shopping for a bra. None of the participants had used an online fitting service. The participants mentioned "jumping up and down to test movement" or "holding in place" as a component of their criteria to test whether a bra fit well or not. For every participant, a bra fit test included understanding how the breasts behave in the bra, whether the bra suppressed unwanted movement or was able to hold the desired shape. Yet, it is interesting to note according to literature and industry, these tests are not included in any bra fit criteria. However, there were clear discrepancies between what the participant considered to be a preferred bra design and fit, versus what bras they actually own and find comfortable or fit well. It seemed the participants lacked sufficient bra knowledge to verbalise the types of bras they actually like, or in some cases they actually favored other types of bras, but wear certain bras due to issues finding the preferred ones. The discrepancies between the results in the interview and the results in the questionnaires may mean that the participants may not actually recognize what they find most fitting in a bra, or that they may think they like a certain feature in the abstract but when analysing the physical bra itself it may differ. Additionally, since the interview and questionnaire sessions were conducted at two different days, the participant may have changed their mind or approach to their bra opinions. The interview was conducted first and the questionnaire second. In the interview session the subject of bra fitting and preferences was introduced, thus participant's questionnaire responses may have been influenced by having already discussed bras previously.

Due to the small scale of this research and limited number of participants, it is unlikely any significant claims can be made from these interviews or questionnaires. However they do offer a glimpse of an alternative to purely literature or industry based research. The casual nature of these sessions offered invaluable insight to real young female bra wearer's experiences with bras and is a significant part to understanding bra fit and design for this research. Nonetheless, they highlight real problems, namely no single participant was completely satisfied with her bras or current bra fitting systems/method, and skeptical of even trying alternative online bra-fit method. All participants experience, at some point, levels of discomfort and ill fit. 

C H A P T E R 06

DESIGN THEMES 
웅 
From earlier discussion, it is easy to see bra design is a highly complex system that encompasses broad fields of disciplines. A bra system is rather complex with many intricate parts, from strap design to breast volume to individualisation to bra purchasing services all to which may require their own dedicated research. However, for the purpose of this thesis three themes have been addressed as the main focus for research. The following three themes, Breast Outline, Cup Shape, and Distribution of Pressure, have been derived based on the literature search (Chapter 02), as well as through the data collected from the participant interviews and questionnaires. Each theme has been validated by the results of background research, and addresses bra concerns expressed by the participants. These themes help frame the design work and develop appropriate design responses for the research. 


\section{BREAST OUTLINE}

Identifying and understanding the shape of the breast outline (figure 2.9.) is one of the most important factors influencing a good fit for a bra (Lee et al., 2004). Not only does each woman have a unique breast outline, but also the breast outline directly influences how the bra fits against the body, specifically the fit of the bra cups, underwire line and front band. Therefore, to insure an individual bra fit, these bra features are highly dependent on an accurate breast outline measurement. Several of the bra fit issues raised as the results of participant interview and questionnaire sessions may also be directly linked to lack of breast outline measurement. Participants expressed several fit issues, but the main points can be categorized into three main concerns: breasts would "spill out of the bra", the "underwire[s] cut into the skin", and that the front band did not consider the variability in "distance between each breast". All of these issues could be resolved by an independent analysis and measurement of each bra wearer's accurate breast outline. Breasts that "spill out of the bra" relate to the cup size, but also the cup design or shape which connects the cup to underwire to sidepanel. A better design using breast outline will insure that the breasts are fully encased within the cup. "Underwire[s] [that] cut into the skin" suggests that the underwire does not respond to the curvature of the breast appropriately. Again, a breast outline measurement will offer a better fitted underwire shape to the individual breast curve. Lastly, as the front band is generally designed to be non-adjustable, some women experience the cups to be too far apart or too close. Again, the measurement of the front band can also be related to an accurate breast outline reference.

The literature review on breast outline as a key measurement for bra fit combined with the results of volunteer participants, as described earlier, led to the selection of breast outline as the first design theme. Two design techniques also contribute to this theme. First is the development of a semi-automated breast landmarking and measurement procedure, which are based on 3D scans and parametric software, Rhino Grasshopper. Through breast landmarking points, a breast outline can be drawn and measured, which in turn will be the basis to design an individually fitted bra. Second is the development of an underwire design based on breast outline, using specific $3 \mathrm{D}$ printing and knitting techniques. Both of these design responses are discussed in the following chapter, Chapter 07. 


\section{CUP SHAPE}

Bra cups are a critical feature of the bra. It is the cups, which encase, shape and help support the breasts. However, bra cups often do not adhere consistently to the shape, style, fit or size of breasts, and therefore bras do not follow standardized sizing and can result in an unpredictable fit. Additionally, since the current bra system relies heavily on the bra wearer to fit into an existing bra, versus the bra being fit for the bra wearer's breasts, many women cannot find a bra that fits properly to their body. Participants have also acknowledged this disparity in bra size, such as explaining that a good fit is limited "by what is offered in store". Furthermore, participants have noted that though there is a variety of styles and aesthetics, they are not offered in every size or body type. The results of interviews and questionnaires suggest a non-padded and balconette style bra have been most popular. However, it has been suggested that the non-padded bra sometimes does not offer enough support for larger breasts or shape the breasts in a desired manner.

In order to respond to miscommunication in sizing, the cup has been designed based off the individual breast measurements using the same system as the breast outline from above, through the parametric software, Rhino Grasshopper. Subsequently, the cup style can change and the measurements will adjust to differences, eliminating the need for multiple fitting sessions for each new bra style. Additionally, through experimental knitting techniques, a 3D formed cup design has been prototyped in attempt to respond to issues with traditional nonpadded bra cups. This cup shape is knitted seamlessly to the band and underwire in an effort to stretch and form to the wearer's breast while maintaining a desired shape and structure. This theme spreads over the design processes of Chapters 08 and 09.

\section{DISTRIBUTION OF PRESSURE}

One of the most notable misunderstood concepts is understanding which part of the bra offers the most amount of support for the breasts. It is commonly argued that the cups or the straps are the parts of the bra, which uplift and hold the breasts in shape and therefore the most important parts. However, literature review and industry research suggest the band is the section of the bra, which hold majority of the support (McGhee \& Steele, 2011). The band has been designed to fit quite tightly across the underbust section of the body. This design results in a very high-pressure structure, which may provide discomfort to displeasure for the wearer. Additionally, many bra bands have rigid seams by the side panels, which give the band structure, but they also add to the tightness and the uncomfortable high-pressure feel. The participants in this thesis had all expressed concerns with bra bands being too tight, though they understood that it was purposely fitted tight for support.

Some bra bands, such as Fortnight Lingerie, use a longline band design in their bras to distribute the pressure of tight bra bands, while giving more support from the bottom up. This thesis will use this same feature, and design bands that are long-line style to create an optimal support system, without high pressure concentrated in a small area. Additionally, the design of the bra is composed in a parametric manner, again using the software, Rhino Grasshopper. This means that each component of the bra, such as the cup, the band or the front-band, can also be adjusted as desired all while remaining consistent with the individual's breast measurements. Lastly, through the 3D knitting process, experiments working with knitting structure and yarn choice allows for pressure of the bra to be added or removed. This theme will be considered in Chapters 08 and 09 . 

C H A P T E R 07

BREAST DATA COLLECTION 
This chapter will identify and highlight the necessary parameters and measurement criteria that are essential for designing the bra structure for an individual fit. The essential body data was collected from 3D scanning technology to form a 3D mesh model, this captured images of the breast and upper torso. This model was then input into a parametric modeling software to which the author developed a semi-automated process to landmark and draw the necessary measurements.

The process was developed so that each $3 \mathrm{D}$ scanned model could be input into the software and landmarked accordingly. Using specific Grasshopper definitions, the landmarking points were deciphered, which allowed for subsequent measurements. 


\section{D SCANNING FOR \\ BREAST IMAGING}

3D scanning technology was used to obtain accurate breast images of each participant. Imbedded within the process of $3 \mathrm{D}$ body scanning, there are several methods for measurement purposes: scanning the breasts in the nude state, scanning the desired breast shapes by asking the participant to lift her breasts to where she wants the bra to be fit, and finally, based on the softly supported breasts, asking the participant to wear a soft bra. In this research, nude figure method was selected. Lifting breasts option was eliminated because the determination of participant's desired breast position varies from one to another, and from time to time. Similarly, different soft bra styles create different breast shapes, and consequently wide variations. Nude breasts option, however, gives unique original data that can reflect the true size and natural shape of the individual breasts. Each participant was asked to stand in a relaxed position with their hands on their lower waist, while the author captured the breasts and upper torso data, using the Artex Eva hand-held scanner. Once scan data had been acquired, each scan was examined in the editing software, Meshmixer. During this phase, any unnecessary imaging, such as the participant's partial image of head or arms, were removed from the scanned model. Moreover, small data corruptions, due to minor errors in scanning process (e.g. pin holes in the image), were corrected. 


\section{PARTICIPAN T 3 D SCANS}
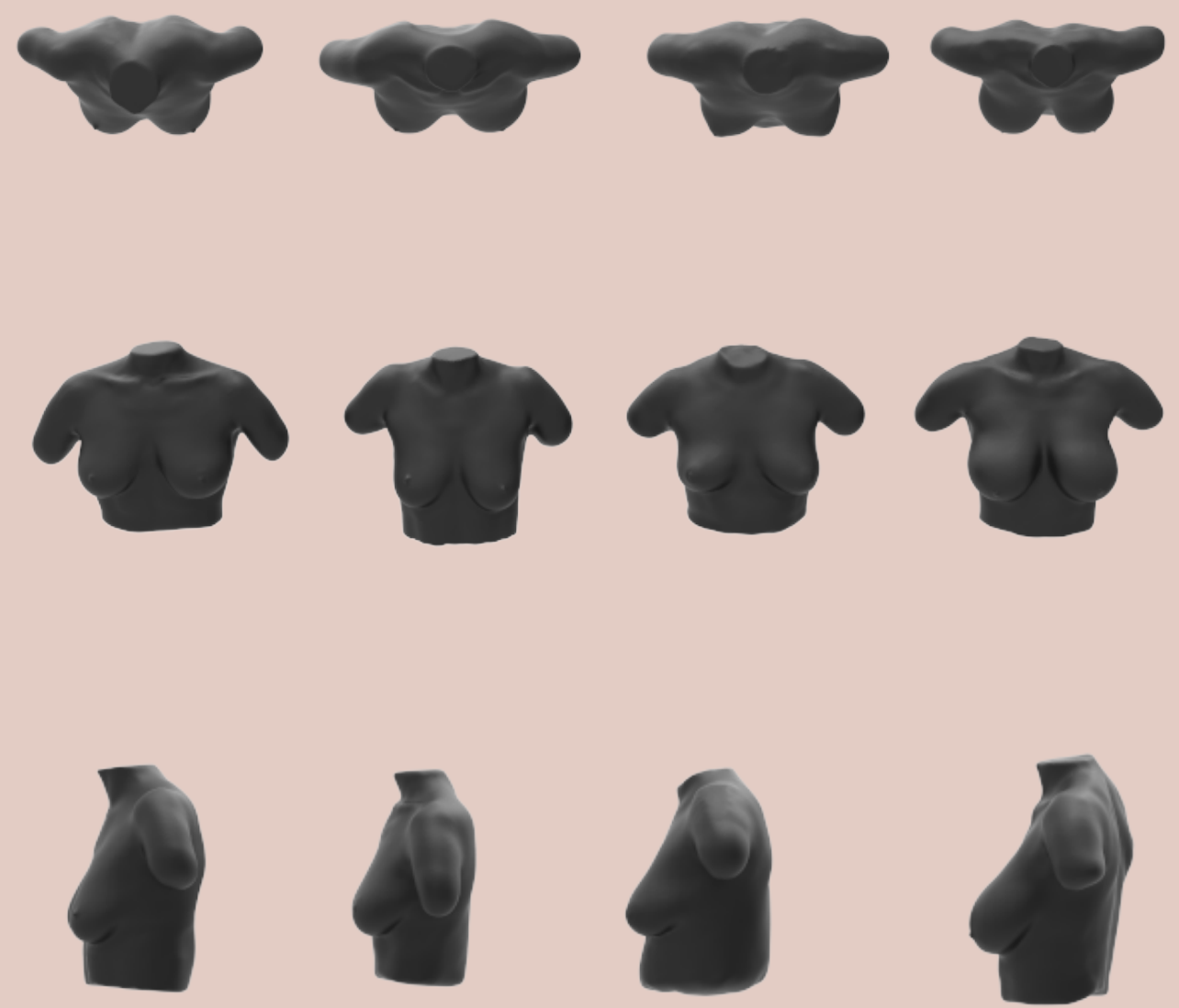

Participant No. 1 Participant No. 2 Participant No. 3 Participant No. 4 


\section{A N D M A R K I N G POI N T S}

(Adopted from the research of, Lee et al. [2004])

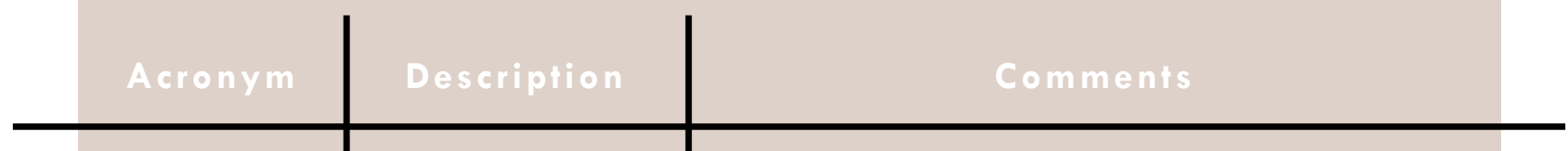

IBP

IBP
(L) and (R)
OBP
Outer breast point
(L) and (R)

The point at which the breast curve ends in the inner front, by the sternum. The positioning of this point may be variable depending on the bra style and design.

The point at which the breast curve

ends, by the arms. It is located on the line connecting the FAP and the SWP. It's location is defined as the midpoint between the FAP and the intersection point of FAP-SWP and the horizontal line passing through the BBP.

It is the point where the breast meets the ribcage, along the inframammary fold of the breast.

Bottom breast point

(L) and (R)

BP

Bust point

(L) and (R)

UBP

Upper breast point

(L) and (R)

UBPA

Upper breast point along underarm line

(L) and (R)

Determined where the nipple is positioned.

Defined, for medical use, as the highest point along the breast line (Lee et al., 2004). It is used to determine the breast outline along with the IBP, OBP and BBP.

Defined, for the brassiere industry, as the intersection point between the line from the CSP to the BP and the line between the two FAP Lee et al. (2004). The positioning of this point may be variable depending on the bra_sty le and_design.

The center point of the length of the shoulder.

CSP

Center shoulder

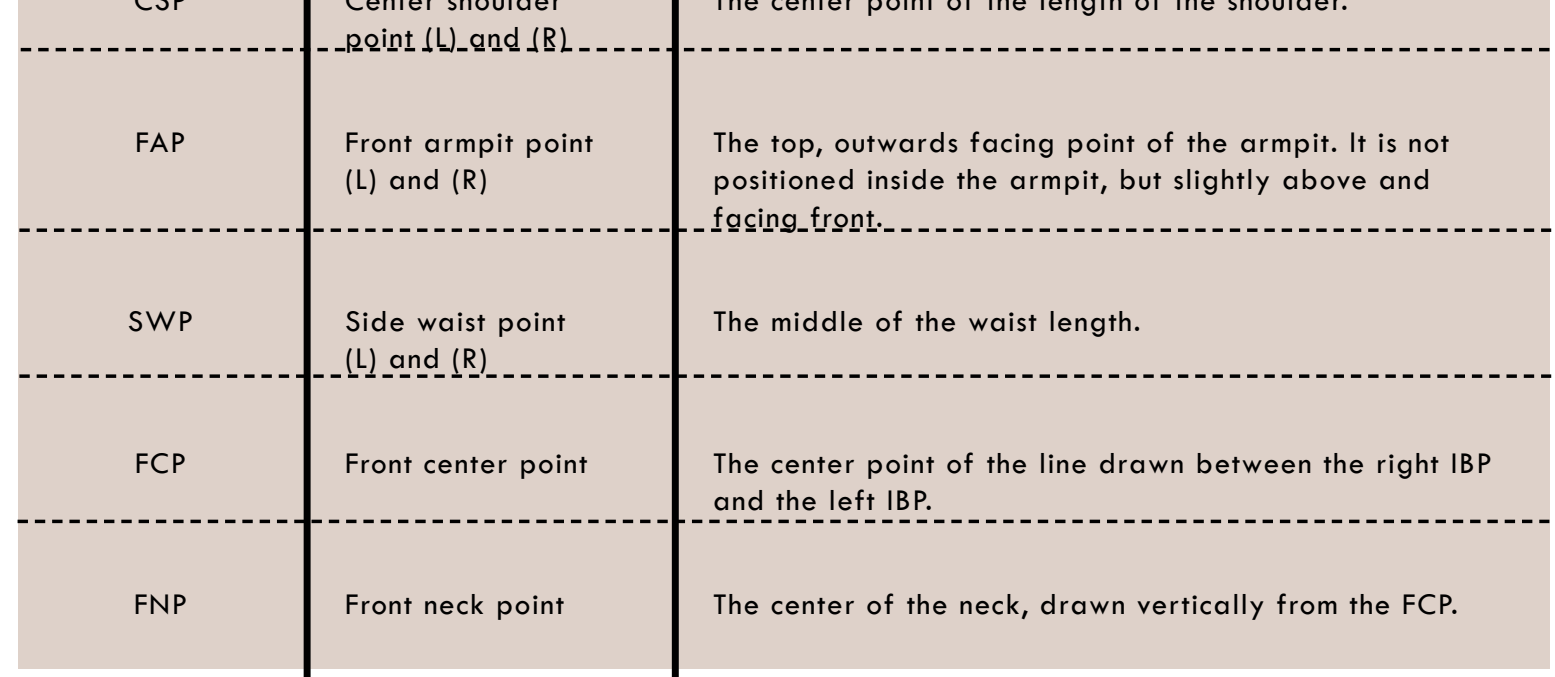




\section{LANDMARKING \\ FOR BREAST MEASUREMENT}

As discussed in Chapter 02, Section III, the landmarking points are a series of points on the breasts that have been validated to extract accurate measurements of the breasts (Lee et al., 2004; Lee \& Hong, 2006). The following measurements will then be the basis of which the bra design can be built.

\section{A D D I T I O N A L L A N D M A R K I N G P O I N T S F O R B R A D E S I G N}

(Author)

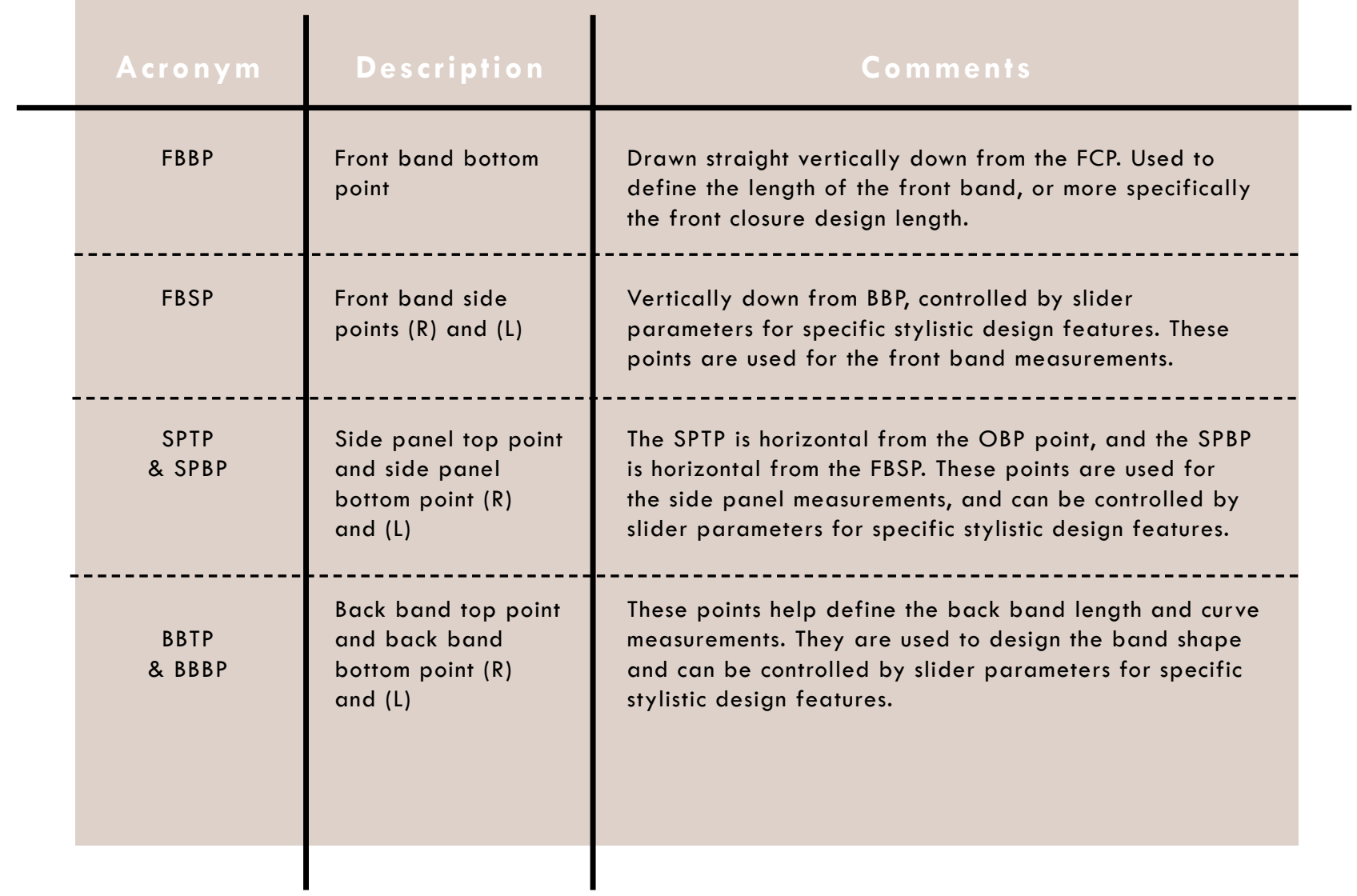




\section{A L L LANDMARKING POINTS}
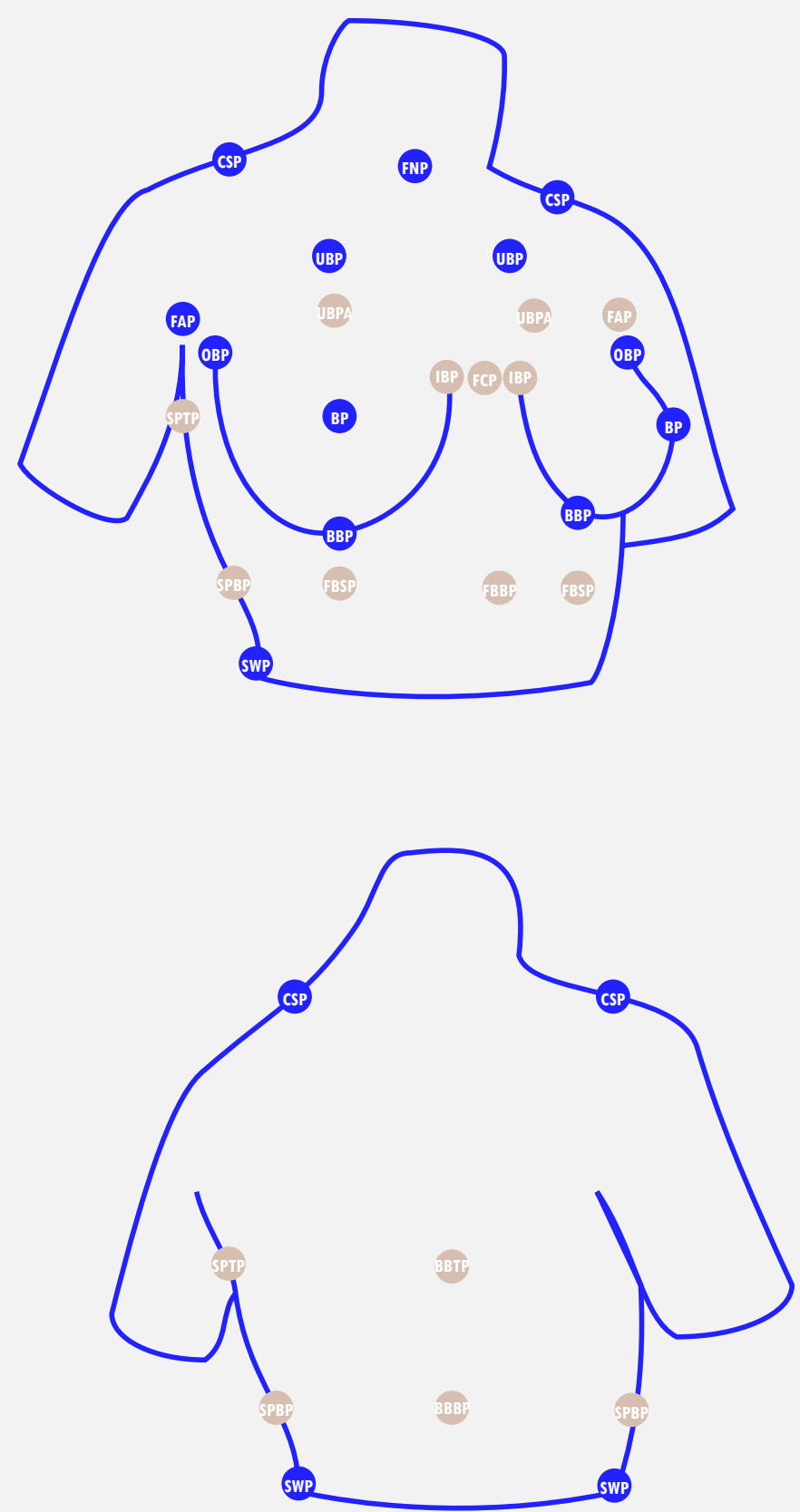
The Grasshopper software is based on programs that are created by dragging components onto a window.

The outputs to these components are then connected to the inputs of subsequent components. A series of components connected together are known as a Grasshopper "definition". In this case a series of parametric definitions were developed to help standardize the process of identifying the necessary landmarking points, as well as, grouping these points in order to extract accurate breast measurements. The process was such that a scan was input to the Grasshopper program, where first, six landmarking points (figure. 7.2.) were appointed manually. Once these landmarking points were identified on the scan, then additional points were automatically placed according to the designed Grasshopper definition list, determined by the author. The definitions can be turned on and off to call attention to certain measurements. Every measurement result on the scan refers back to the original landmarking points of figure. 7.2. This insures that when a new scan is inputted into the system, the measurements will not need to be re-calculated, as long as the landmarking points are placed correctly and consequently all other measurements should follow accurately as well. Though each scan represents the same landmarking points, it is clear that the positioning is slightly different for each case. When these points are used in the necessary combinations to make up the breast measurements, as explained later in figure 7.7., it starts to become apparent how each breast contour is unique. Though some points and measurements for a bra design will be static, such as the underwire line, many measurements will be highly variable and dependent on the bra style and design itself. For example, the breast outline depends on four landmarking points, IBP, UBP, $\mathrm{OBP}$ and BBP, all of which are constant in positioning. The actual cup design depends on, the IBP, UBPA, $\mathrm{OBP}$ and $\mathrm{BBP}$, where the UBPA positioning is variable depending on what type of cup style is preferred by the bra wearer. 
Participant No. 3
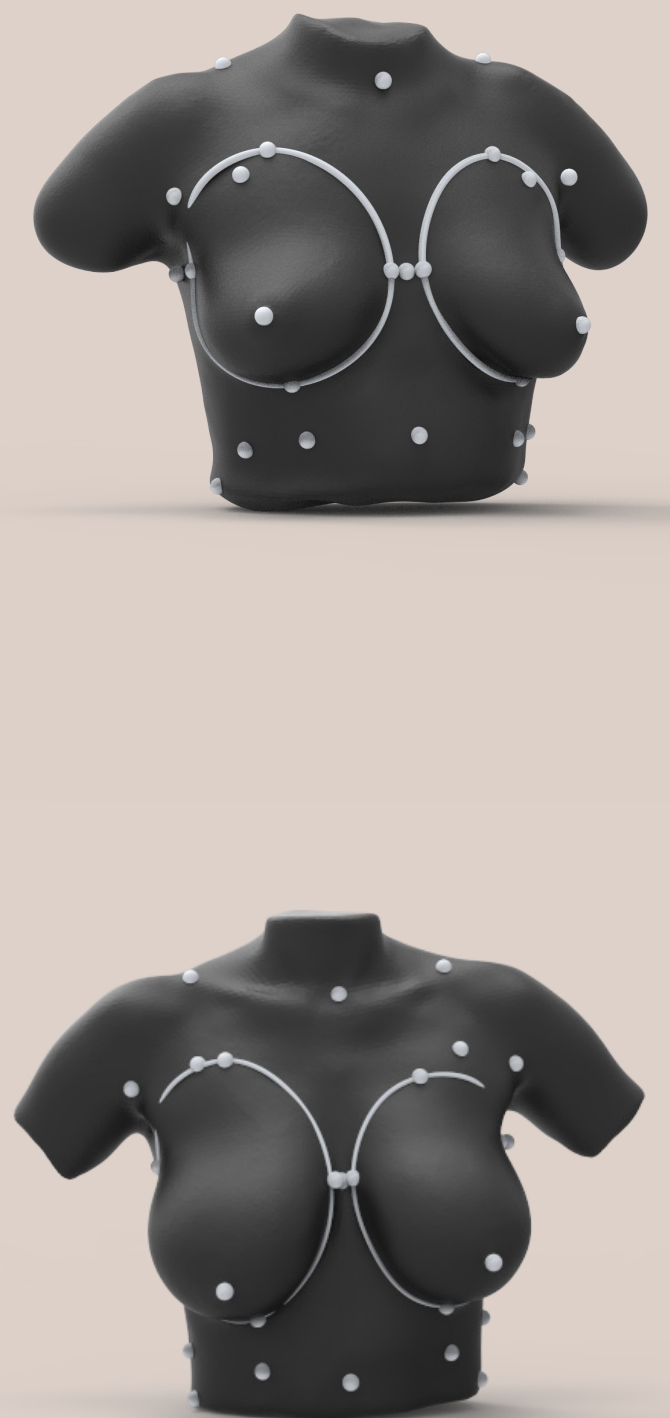

Participant No. 4 
The points IBP, BBP, BP, UBP, CSP, FAP, SWP, and FNP, are points that are manually determined by the descriptions and past research of Lee et al. (2004). Using these manually referencing, the following points, OBP, UBPA, FCP, FBBP, FBSP, SPTP, SPBP, BBTP, and $B B B P$, are obtained and developed to be marked automatically using the parametric measuring system described. The automation of the points insures that the measurements for bra design are adapted to the individual 3D scan that is inputted into the program.

\section{O U T E R B REAST P O I N T S (OB P)}

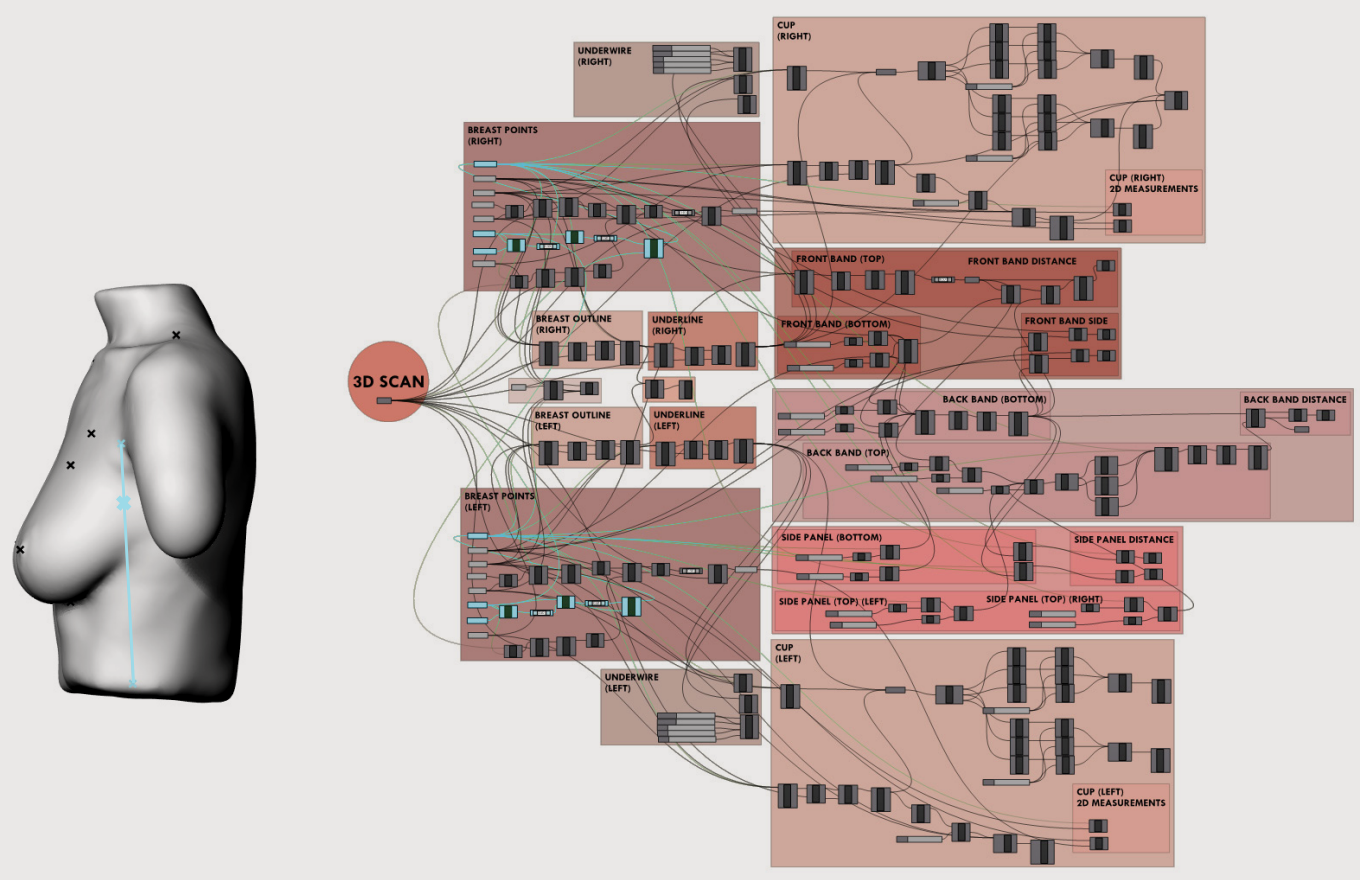


U P P E R B E A S T P O I N T S A ( U B PA)

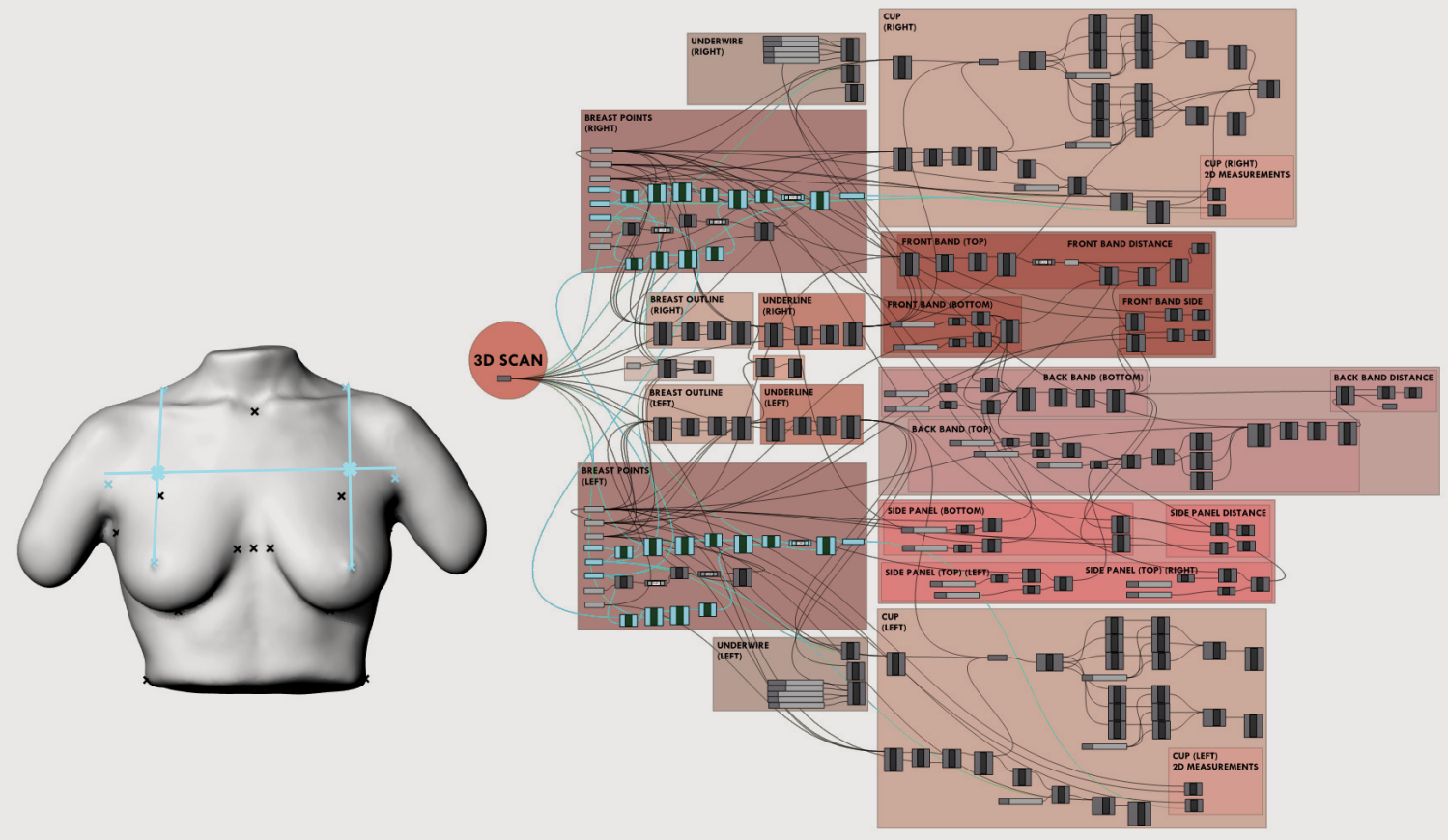

FRON T C E N T ER P O I N T (F C P )

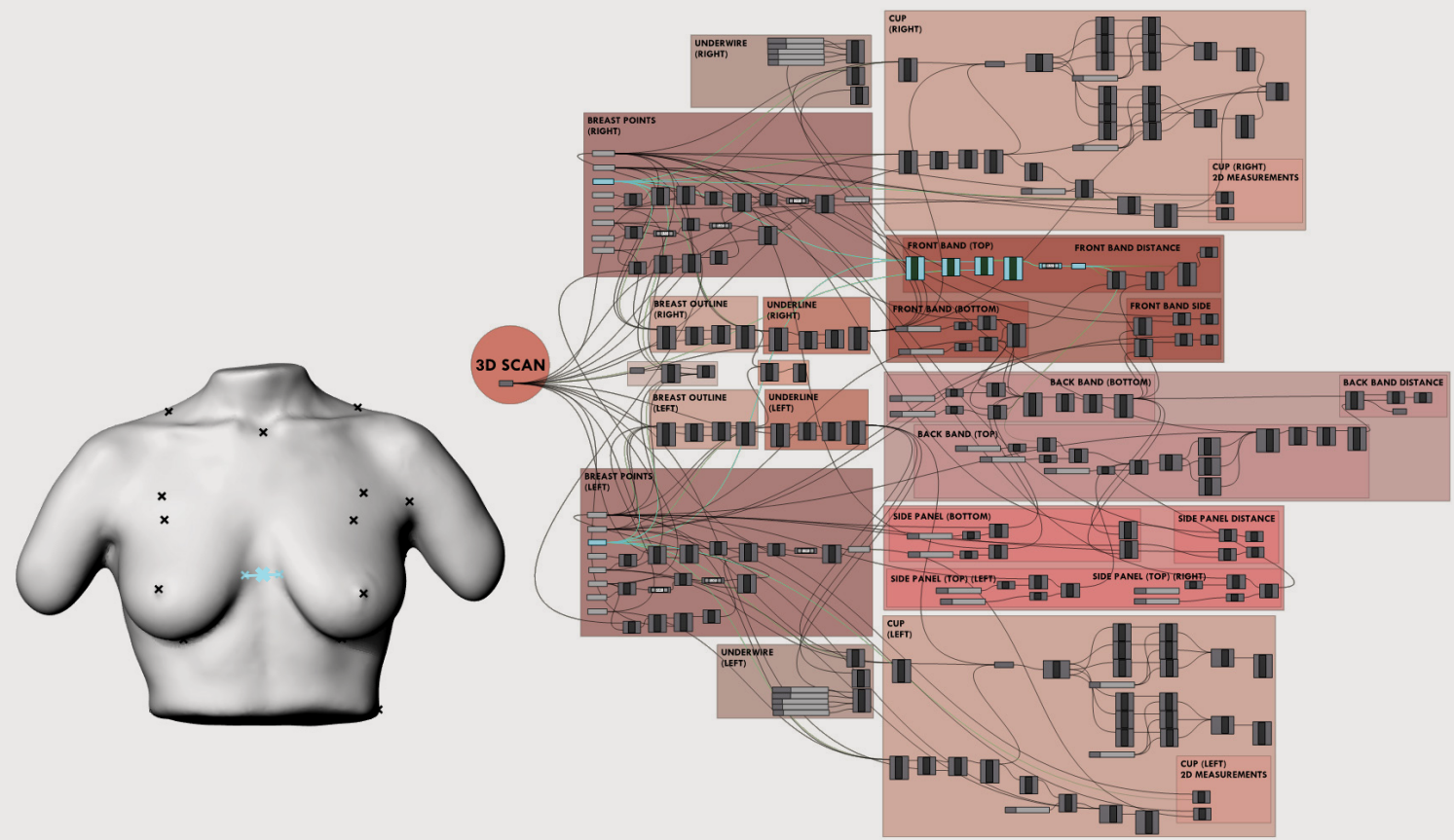




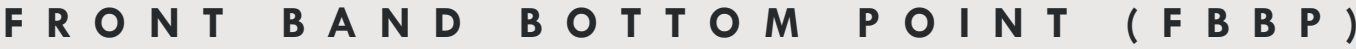

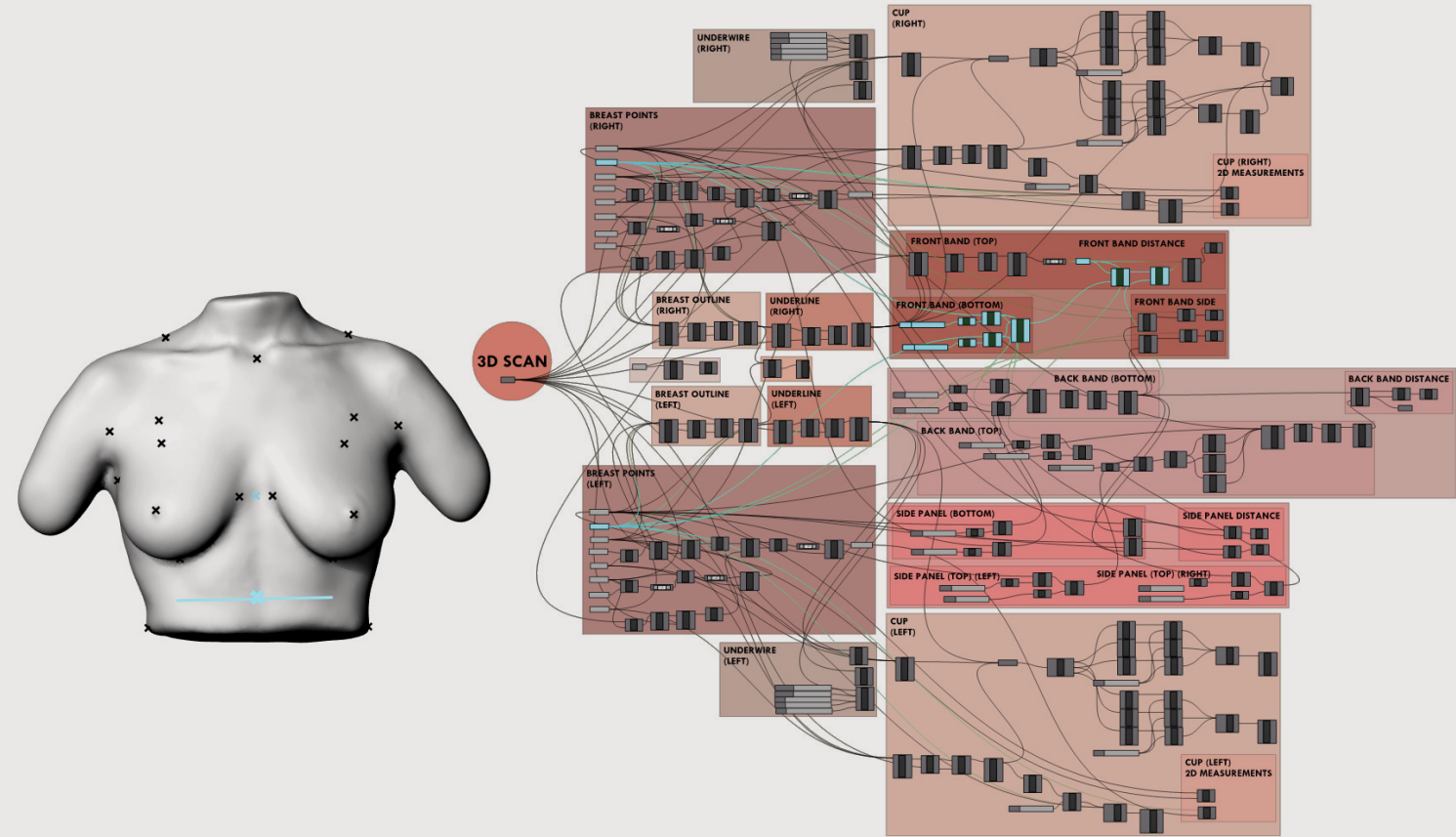

F R O N T B A N D S I D E P O I N T S (F B S P)

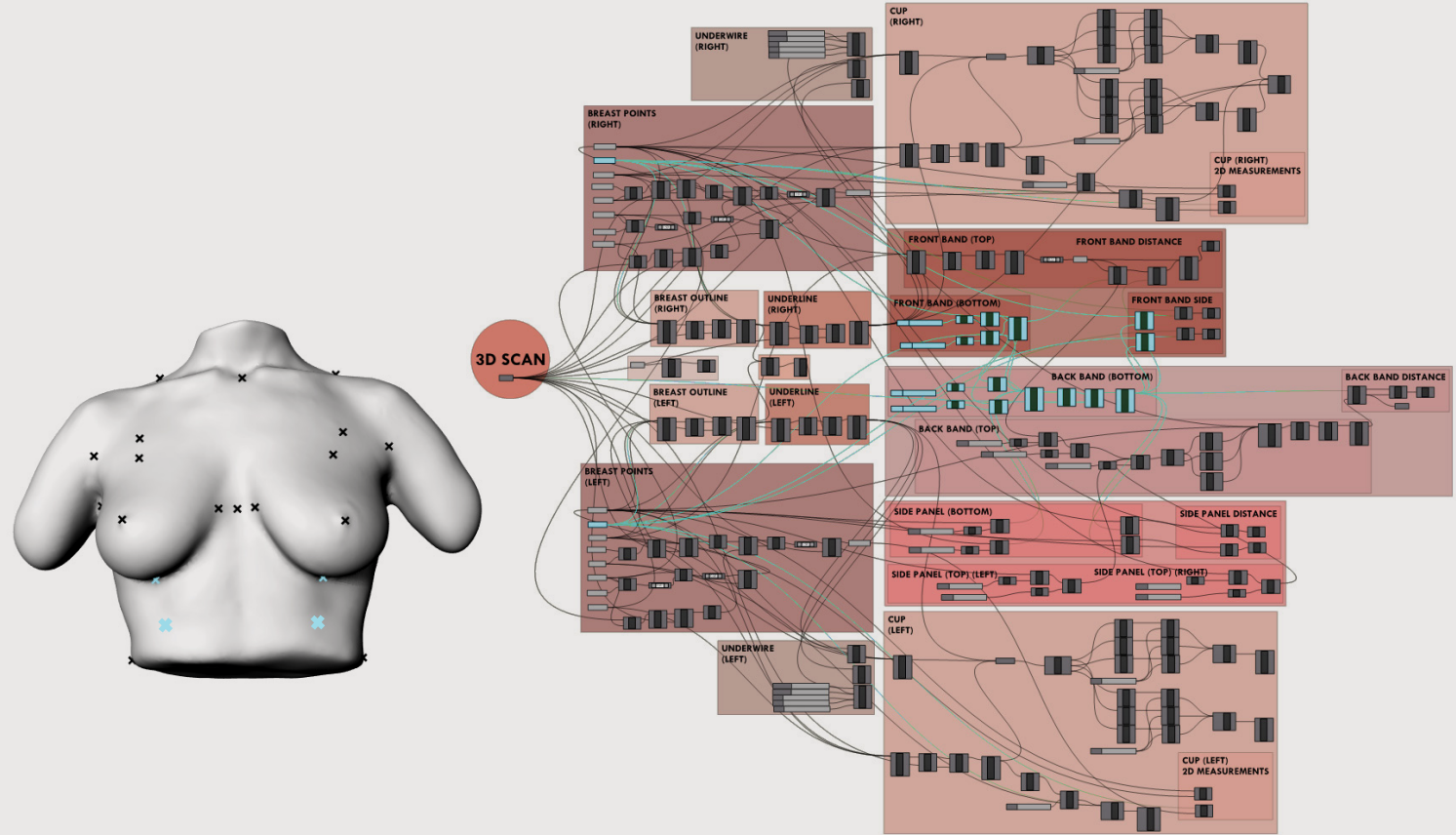


B ACK B A N D POIN T S (B B T P) (B B B P)

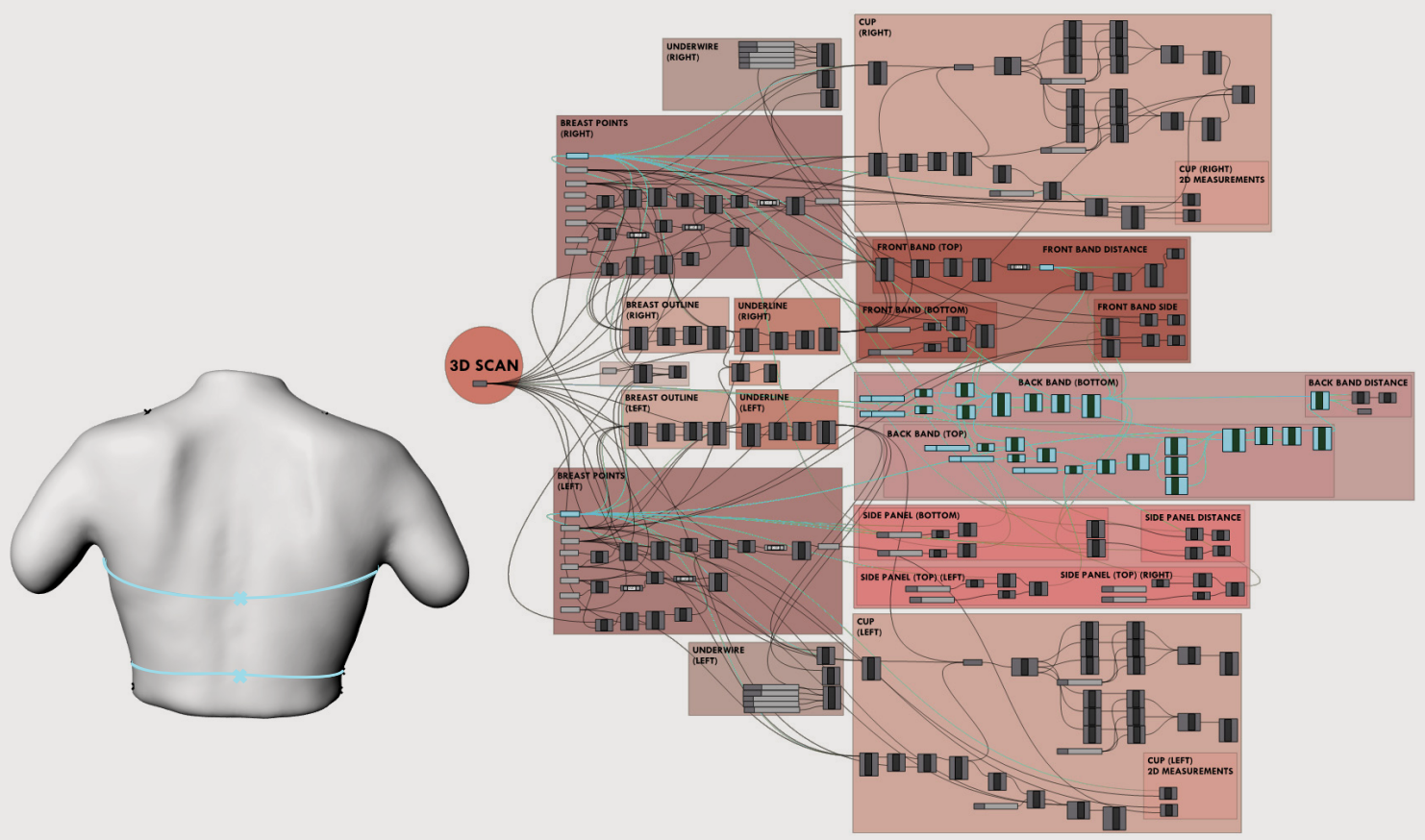

S I DE PANEL PO INTS (SPTP) (S PBP)

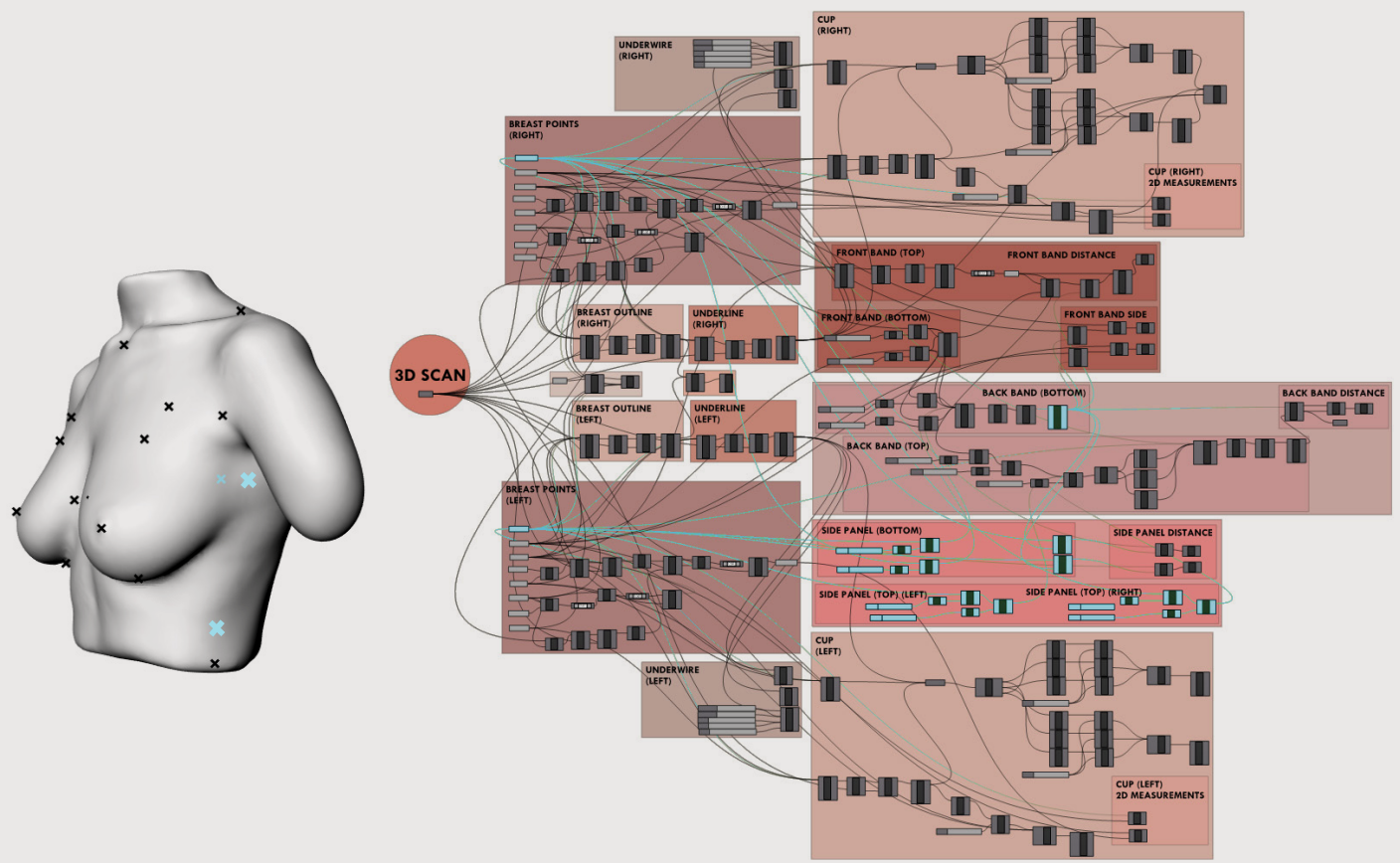




\section{DRAWING BREAST CONTOUR COMPARISONS}

Once the landmarking points have been established, an analyzing technique could be used to compare the breast scans. This research asked for participants of self-identified bra size, $12 \mathrm{D}$, but as noted in Chapter 05 , each participant explained that the bras they fit into were actually within a wide range of sizes. This meant that though every participant, at some point, was wearing the same size as indicated on the label of the bra, they were also wearing a range of sizes that were different from each other.

Through these landmarking points, the author was able to draw outlines of the different contours of the breast shape in order to further examine these variances (figure 7.7.). The first set of sketches outlined the points OBP to BBP to IBP for each breast, where a line is drawn through these points to create an underbust line, a line that is used to measure and design the underwire shape. The points between the two IBP are also connected in this sketch to outline the front band distance. The second set looks at the points drawn from OBP to BP to IBP for each breast. This can be understood at the bust point line. When the first set and second set are combined and viewed from a perspective angle it becomes quite interesting. Here, the differences between bust heights are examined. One can notice that each participant has a different bust point (BP) altitude and angle. Moreover, for each participant there are different BP heights between the right and left breast. This suggests that the line between OBP to BP and IBP to BP is unique not only for each participant but for each breast which would impact the measurements of the cup design as well as the side panels. 
Participant No. 1 Participant No. 2 Participant No. 3 Participant No. 4
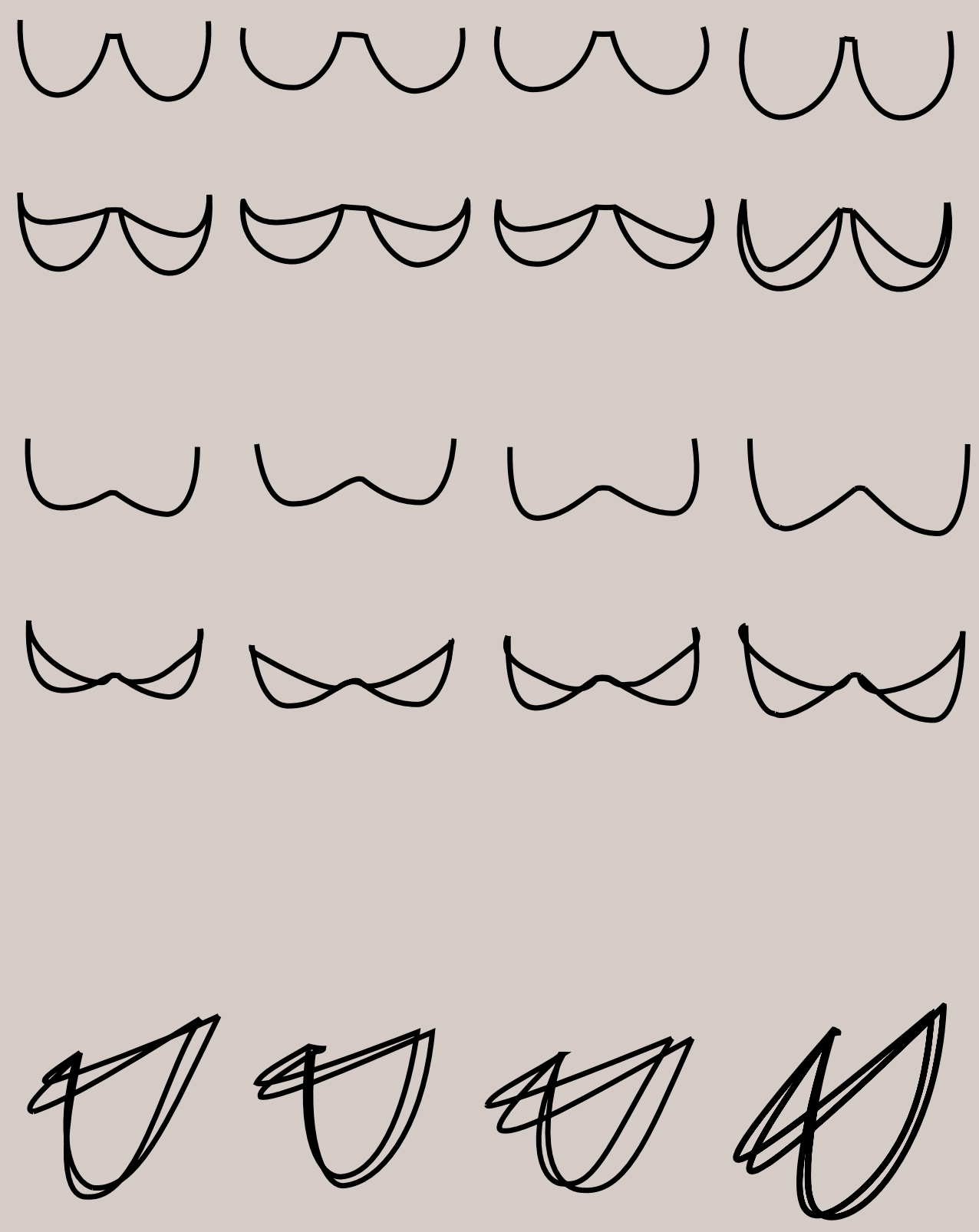

BREAST CONTOURS 


\section{"Underwires seem to be outdated and limited in design, variation and personalisation. Often too stiff, intrusive and creates discomfort! Need to create something adjustable and more in tune with the female anatomy."}

- Participant No. 1

Underwire from breast outline experiment

As mentioned earlier, the breast outline is important for understanding the breast shape and thus good fit into a bra the breast outline must be measured. The measurements of the breast outline were used to design the underwire (when the bra design requires one). It has been noted that the underwire being used in traditional bras is inconsistent with the actual breast outline shape of the wearer. Again, this is due to the fact that women must fit into predetermined shaped bras. Nevertheless, the commercial underwire is $2 \mathrm{D}$ and cannot accurately shape the breast, especially since each breast is shaped differently. 


\section{BREAST DATA COLLECTION}

\section{R I TER I A A S S E S S E D}

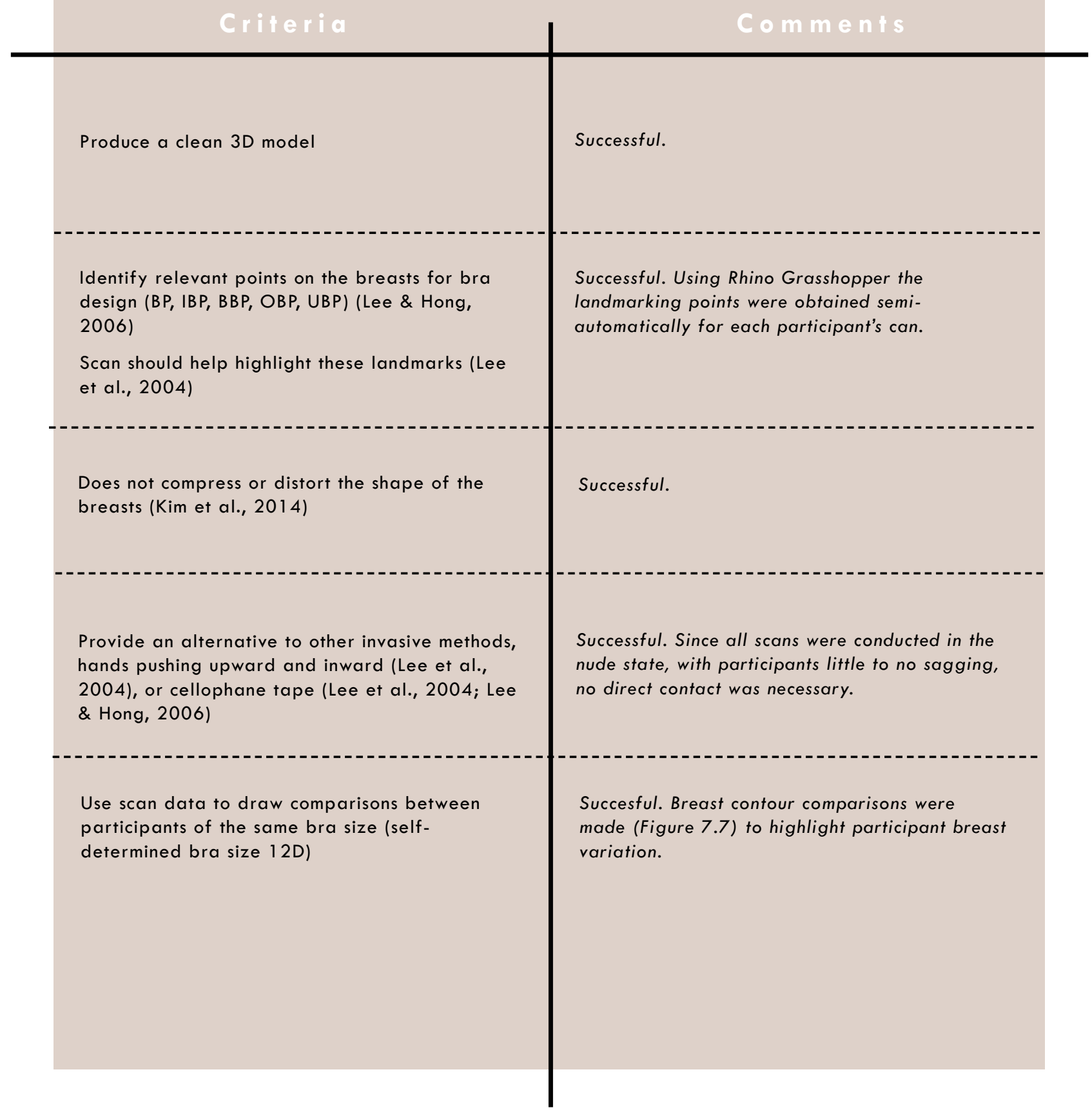


CHAPTER 07

BREAST DATA COLLECTION

DISCUSSION

$\vec{\omega}$ 
Traditional bra sizing is standardized in which bra wearers must fit into predetermined sized bras. These experiments, using landmarking tools to analyze breast shapes and contours through measurements drawn, demonstrate the variance in each participant. The scanning, however, was limited by the type of scanner used, the hand-held scanner. Though the scans acquired necessary body imaging data for every participant successfully, the requirement for a person (in this case, the author) to operate the handheld scanner, can potentially add a level of discomfort for the participant. In an ideal scenario, in a commercial setting, this phase of the bra system would involve an automated body scanner, such as the body scanners provided by, Shape Analysis (UK), which would automatically capture the body data without an external person operating the scanner, which would provide a great deal of privacy for each person. However, for the purpose of this research the Artex Eva hand-held scanner, which was readily available at the university and is relatively manageable to operate, was perfectly adequate. Three out of four of the participants did not mind the scanning scene, while one of the participant would have preferred an even more private setting. Though the process of obtaining the scans and setting was not optimal, the resulting scans provided "clean" data to work with. The landmarking method used for this thesis derived from the work of Lee et al. (2004). The author translated his methods for acquiring the landmarks in a semi-automated system in Rhino Grasshopper. This way each scan does not have to be landmarked manually, but instead can be input into the Grasshopper scene and have the appropriate landmarks and the measurements they produce appear against the scan. This method worked quite well to produce clean and useable scanned digital models that were able to provide a base for developing measurements based on landmarking points, even though it was limited by the initial manual landmarking necessary. Ideally the scan and software associated would be able to recognize more obvious landmarks, such as the BP or the CSP, which would accelerate the entire process and make these initial landmarking points to be more accurate. Of course, another benefit of landmarking and measuring through the designed Grasshopper system is that each landmark can be adjusted, which allowed the author change a part of the bra design without having to alter the rest of the bra as well. 

C H A P T E R 08

BRA DESIGN DEVELOPMENT: 3 D KNITTING EXPLORATION 
A research through design approach (Martin \& Hanington, 2012, p. 146) is discussed in this chapter to develop a bra prototype that utilizes 3D knitting manufacturing methods. In order to do this, the iterative process of developing knitted prototypes was spent with a professional 3D knitting technician. Together, over twenty iterations were developed to reach an understanding of 3D knitters capabilities in developing a knitted bra piece with innovative features. 


\section{INTRODUCTION TO 3D KNITTING}

The 3D knitting machine used for the following iterations in this chapter is the flat-bed SES-S "whole garment" knitting machine. These industrial knitting machines allow for a fully automated computer controlled method of production. However, the technical parameters of what can be knitted on the machine are dependent on the machine's capabilities and will determine the type of forms that will be produced.

In order to take advantage of the capabilities of this machine, the digital knit programming must also be understood. In order to knit with the Shima Seiki knitting machine, the first step is to programme the design. This involves a series of steps to translate the envisioned design into the computer code using the Shima Seiki CAD system, the data is then saved on a disk and transferred to the knitting machine itself (Underwood, 2009, p.

41). Currently, this digital knit programming has been largely in the domain of the knit technician. However, as the technology for the digital programming becomes more sophisticated and user friendly, there will be an opportunity for designers to enter this technical domain and engage in a discourse in relation to industrial knitting (Underwood, 2009, p. 41).

\section{Basic knit structure experiments}

To gather a better understanding of the 3D knitting machine's capabilities, a variety of small experiments highlighting various knitting structures were performed. All experiments (excluding the spacer sample) were conducted with $100 \%$ merino yarn as this yarn can be knitted without difficulty (some yarns, such as rayon or viscose, need closer attention while being knitted as they are more prone for the thread to intertwine, or are easier to tear while being knitted). Each sample was designed on the Shima Seiki software as the same size square shape. The resulting dimensions of each sample varied depending on the knit structure, which demonstrated the influence of knit structure on size and material properties. A rib structure has a great horizontal stretch, and the resulting physical sample is much wider, than, for example, a plain knit, even though they were both designed in the software as the same square size. These experiments helped develop a basic understanding of how the knitter knits, as well as the relationship between the knitting software and the resulting physical output. 


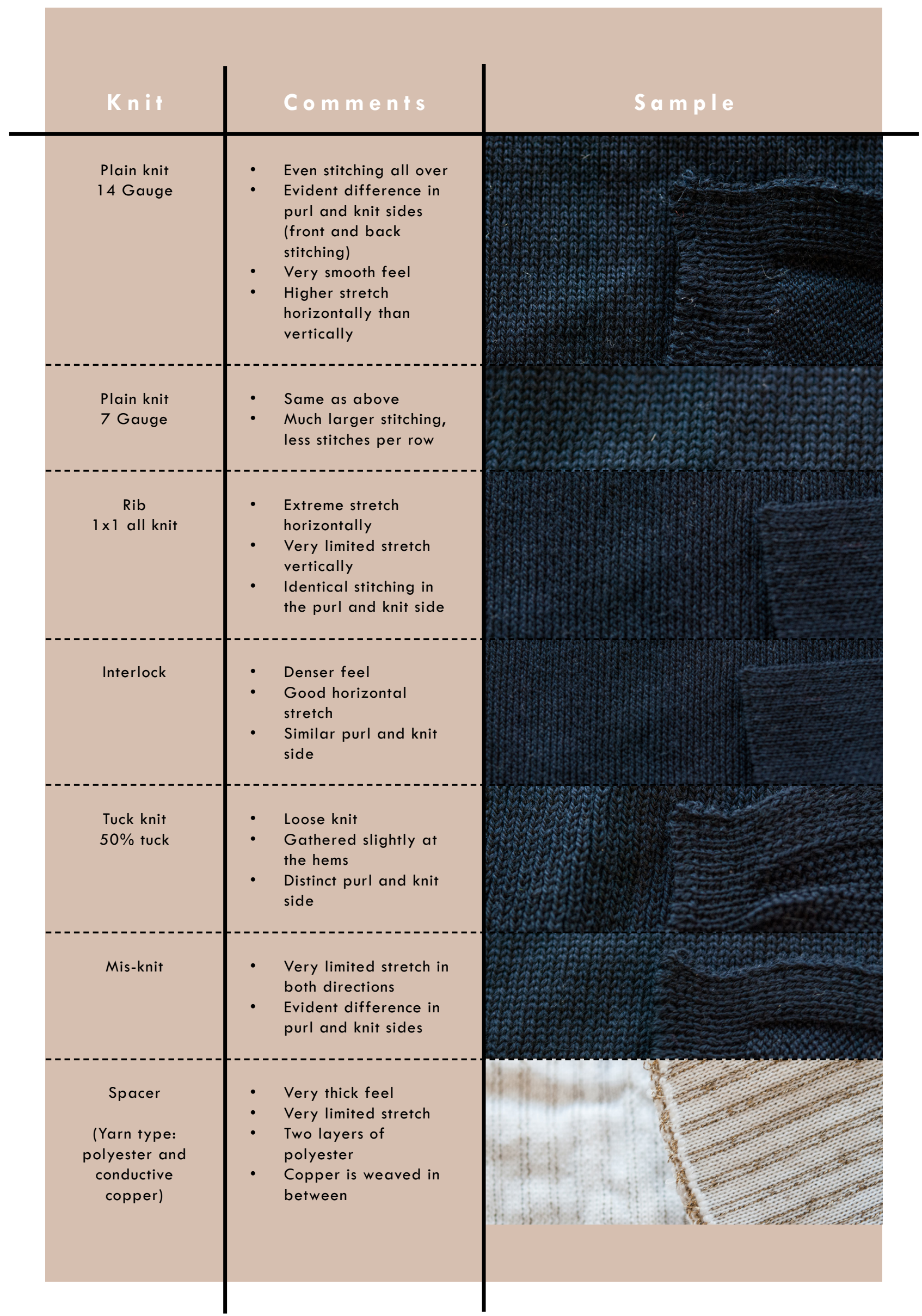




\section{Knitting a 3D Form}

This research aimed to push the boundaries of what has sought to be designed using 3D knitting technology in terms of bra design. Though previous seamless bra designs have been constructed using a similar manufacturing techniques to the 3D knitter, the author has been yet to gather any literature on the development of a knitted bra design that utilizes a seamless 3D shaping in its structure. In her research on knitting 3D shapes and forms which utilizes the same machine technology as this thesis, Underwood (2009) notes that there are limitations for designers to engage in authentic design of 3D knitted form due to the current setup and management of $3 \mathrm{D}$ shapes (by the suggested garment production templates, which are always based on 2D shapes like traditional manufacturing methods) (p. 54). The current digital knitting design software is setup so that the user select a template form and adjust or alter it as needed to generate their own design. The "template" system establishes an easy translation between designing in the software and the capability to knit it using the machine. However, as suggested by Underwood, these templates do not incorporate the development of 3D form (though the 3D knitter has the capability to knit this way). Underwood (2009) explains that for Shima Seiki and similar technologies, measurement and alterations are limited to $2 \mathrm{D}$ pattern pieces which is similar to a traditional 'cut and sew' production method (explained in Chapter 2) (p. 54). With this system, the widths and lengths of the piece can be changed but does not allow for the actual garment form to be altered in 3D (Underwood, 2009, p. 56). Currently, the software and programming process for the knitting machine is specific to the needs of the garment industry. Forms, templetes and tools are based off specific garment types, such as, a sweater, a shirt or socks. This means that development of a design (shapes and forms) is controlled by the knitting machine manufacturer (for the purpose of knitwear garments) and not the designer. Forms that fall outside of the range of garment production templates require the high expertise of the knit technician to navigate around and experiment alterative ways to build up a design. Such designs became complex and costly, in terms of time, programming requirements and the trial and error involved in sampling. (Underwood, 2009, p. 164). 


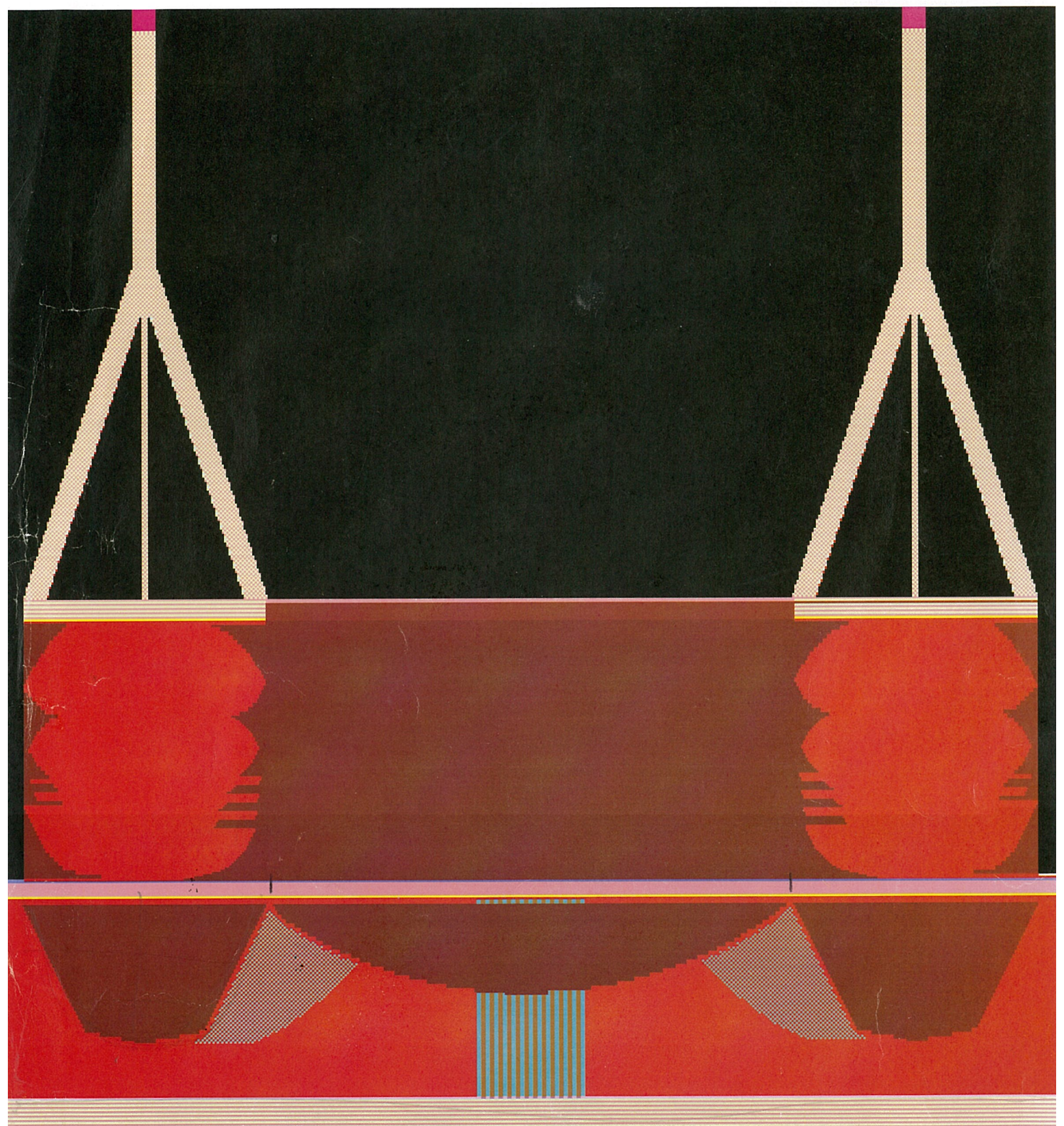

Digital knit design programming corresponding to

figures 8.6.0. - 8.6.1. 


\section{KNITTING ITERATIONS}

The following section outlines a series of iterations developed to knit a 3D formed designed bra garment.

This design process is based through an iterative design process (Karjaluoto, 2013, p. 150) in which each knitted iteration inspires future alterations and design decisions for the next. The design on the bra must be able to correspond to the abilities of the 3D knitter, and these iterations demonstrate how the design evolved accordingly. The bra was based off an almost seamless design, meaning that hooks were still considered to close the band. The bra was knitted flat but allowed for unique seamless designed knitting features, such as tubular knit structures, and the development of a 3D cup shape. Prior to the following prototyping series, it was discussed with the technician that this bra design would be most viable through knitting in the flat-bed knitting machine. This chapter demonstrates a sample of the knitted iterations, a full list and imagery of all knitted iterations may be viewed in Appendix II. 


\section{Initial shape}

\section{Series 1}

The first step towards knitting the bra piece was to understand the initial shape of the band and underwire curvature. Since this bra piece is a complicated form that cannot be adopted from a Shima Seiki digital design software template, it must be remolded into the software completely "free hand", in which the technician draws each stitch himself to create the desired design. This piece was used to quickly identify successfully fitted curves that would be later furthered developed. Merino yarn was chosen at the beginning of the design process, as merino is a uncomplicated yarn to manipulate and stitch. The next two iterations featured the addition of the bra cup sections. Though at this point there was no structuring for a $3 \mathrm{D}$ form, it was an attempt to capture the outline and seamless curvature from the band to underwire to cup. The underwire is demonstrated through a tubular knit structure, however, in these prototypes the tubular knit is too small to even slot in a wire.

\section{Initial yarn selection}

\section{Series II}

The initial design decisions for the bra were based on knitting a highly flexible material piece. A knit that could stretch a lot to allow for comfortable body movement but with a high recovery to insure the material gave enough support. Thus, experiments with polyester yarn was applied to the band and elastane yarn was applied to the cup. However, after being steamed the piece shrunk dramatically and did not add to durability of the garment. It was also clear that the elastance teared easily and did not hold its shape well. The polyester did create a satisfying thin and flexible fabric, though did not contain a very soft feel. Polyester was a possibility for further iterations, and pure elastane was not. Though the elastane was very stretchy and flexible, it was a yarn choice that was not desirable for the cups as it is not a breathable yarn and not comforting for the skin. These first tests were important to recognize the high variation on how the yarn will react after being knitted. The panel can be designed a certain measurement, but after being knitted the yarn choice will change. It is then important to consider how the panel is designed and measurements must consider how the yarn will react to being knitted. After a basic form was developed, where the cup is starting to project a $3 \mathrm{D}$ form, the band is seamlessly drawn out from the cups, seams were added to the top of the band, cup and the bottom to cleanly finish off the edges. Polyester yarn was maintained for knitting the band and merino was introduced for the cups. Merino allowed the cups to hold the structure better than the elastane but also had quite a good stretch and pull. Holes started to appear where the cups meet the band at the outer breast point (OBP) but was adjusted for in the following knitted piece. A tubular hem was added throughout the bottom of the knitted piece. The tubular hem helps achieve a better structure by creating a thicker border. 


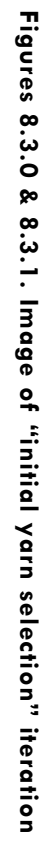
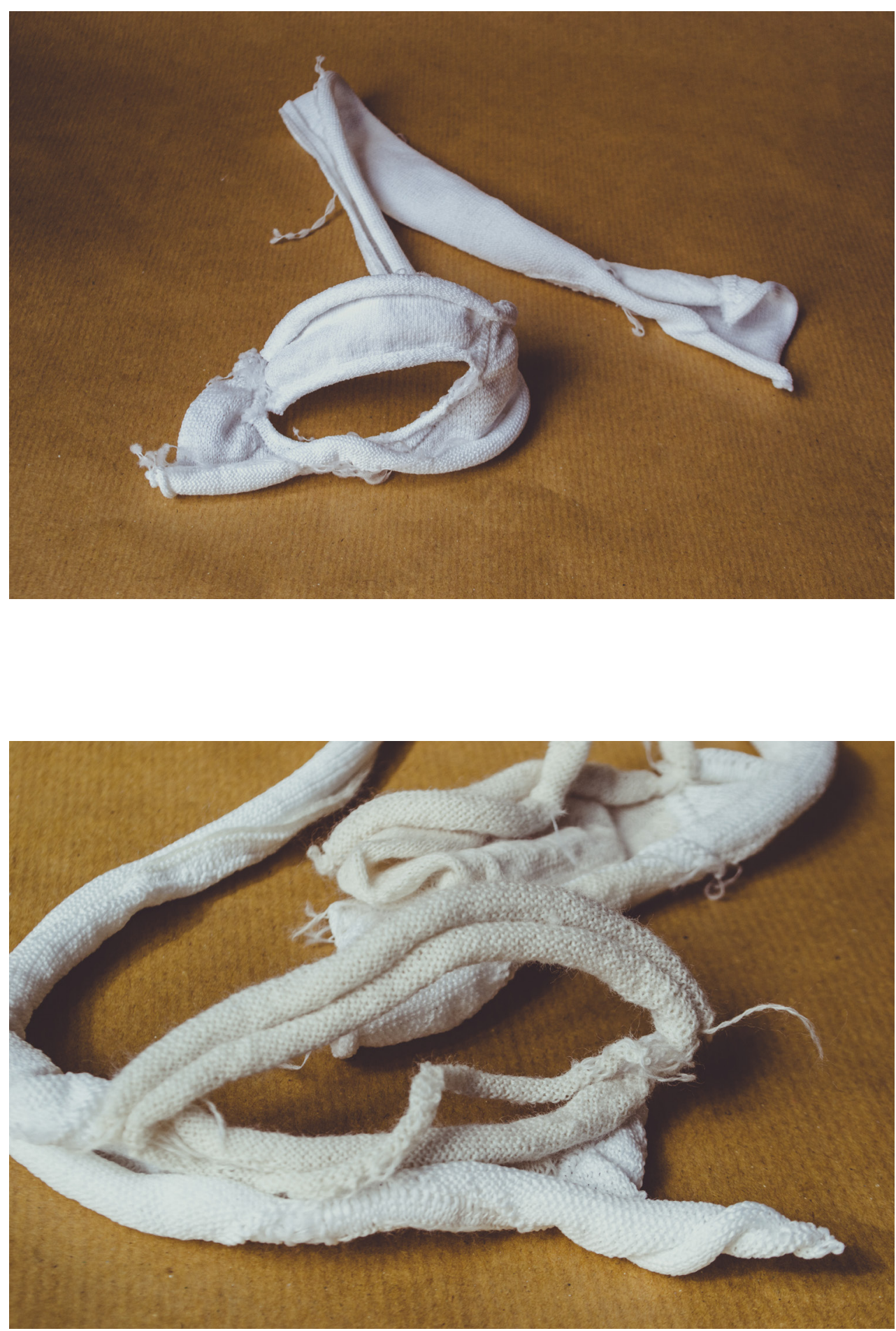

$\vec{N}$ 


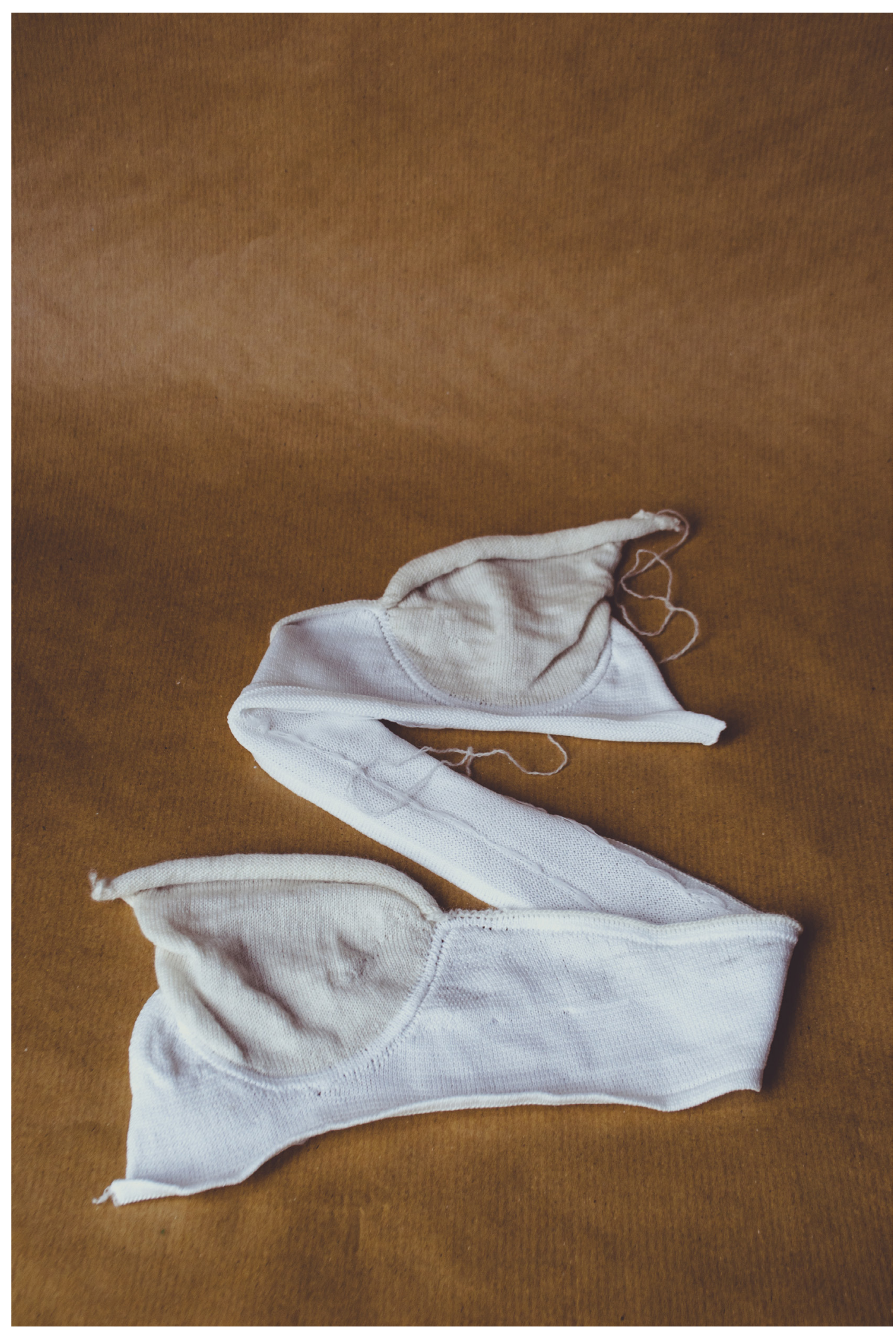




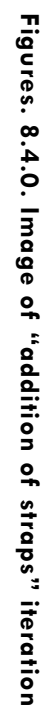

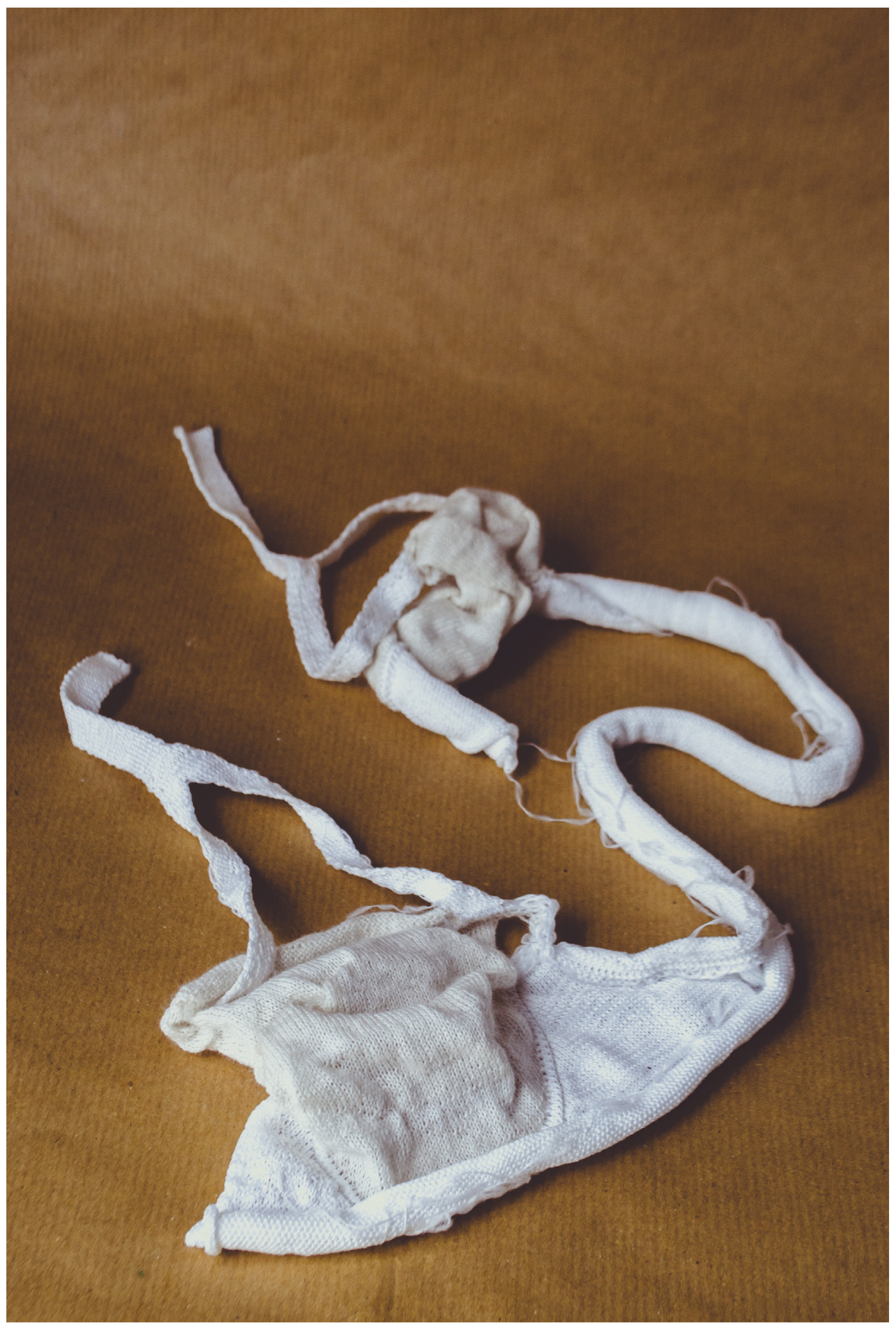

$\vec{D}$ 


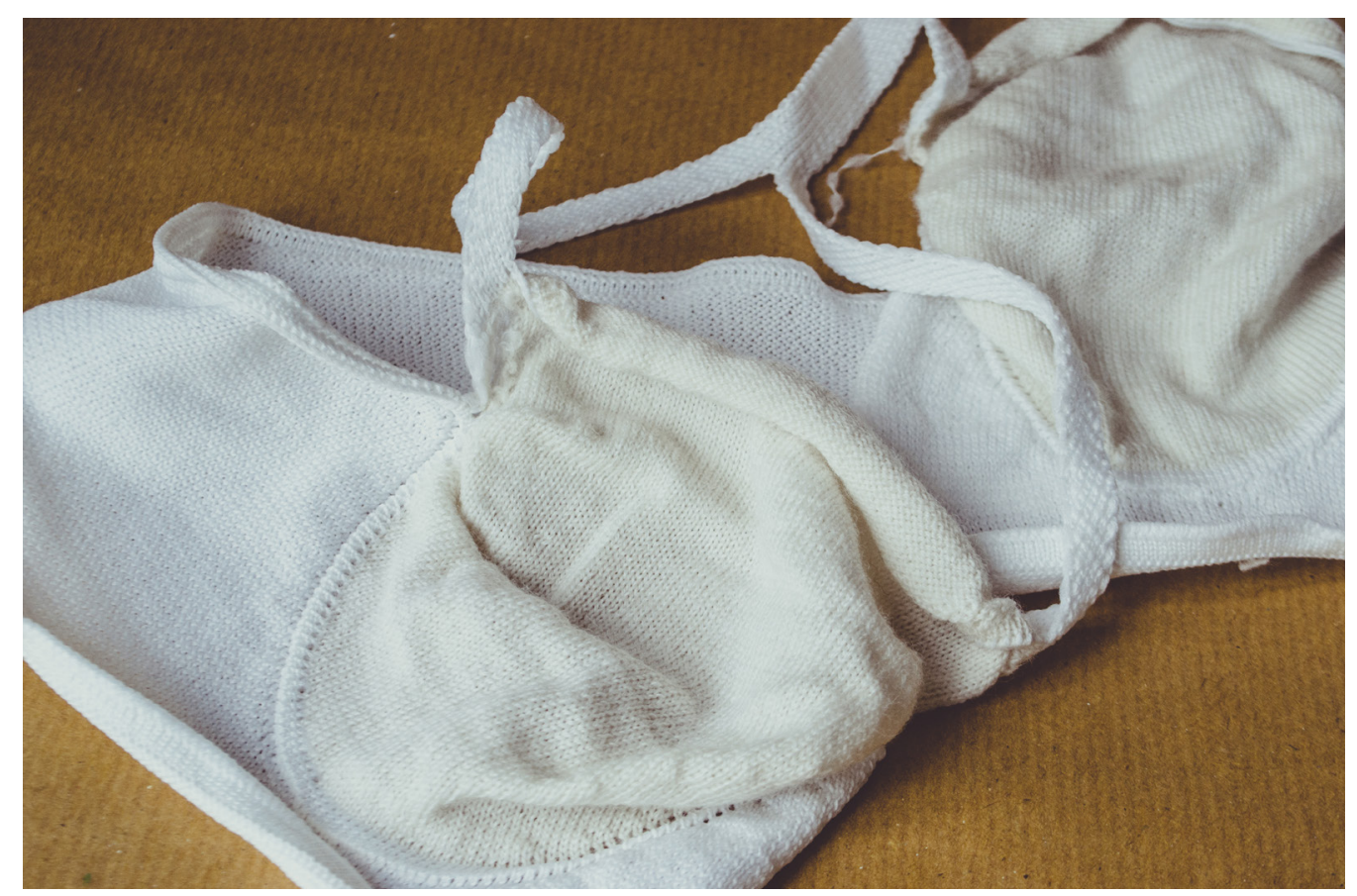

\section{Addition of straps}

\section{Series III}

Addition of straps implemented early on to test the seamlessness of the top end of the cups into the straps. Two straps were designed to come out of the IBP and the OBP. The angle of the strap placement was also examined as this influences the knit structure and the cleanness of the knitting. The number, thickness and detailed design of the straps has the opportunity to be changed in further iterations. This first version of the strap was designed using a $1 \mathrm{x} 1$ rib structure to ensure that the straps would not curl in, and a rib structure is more likely to lay flat. The final two iterations in this series failed to knit, as there were issues with knitting the cup edges into the band seamlessly. The cup edges kept having problems at the bind off, which was creating undesirable holes or loose stitches at the corners. As well as the cup at the inner breast point is creating a straight line up instead of seamlessly curving down to meet the front band. The strap placement, where the straps connected into the cups was adjusted to see if this could help with a cleaner and smoother knit. The outer breast point where the cup meets the underwire line was not seamlessly knitted at this point. Each time a panel was knitted, a hole or very loose stitching was created. It seemed that the front bed needles pulled when being held as the back bed needles knit. This caused too much stress for the front bed needles and thus the holes or tearing appeared. Adding a line of plain stitch before the new knit structure was introduced was applied to resolve the issue. 


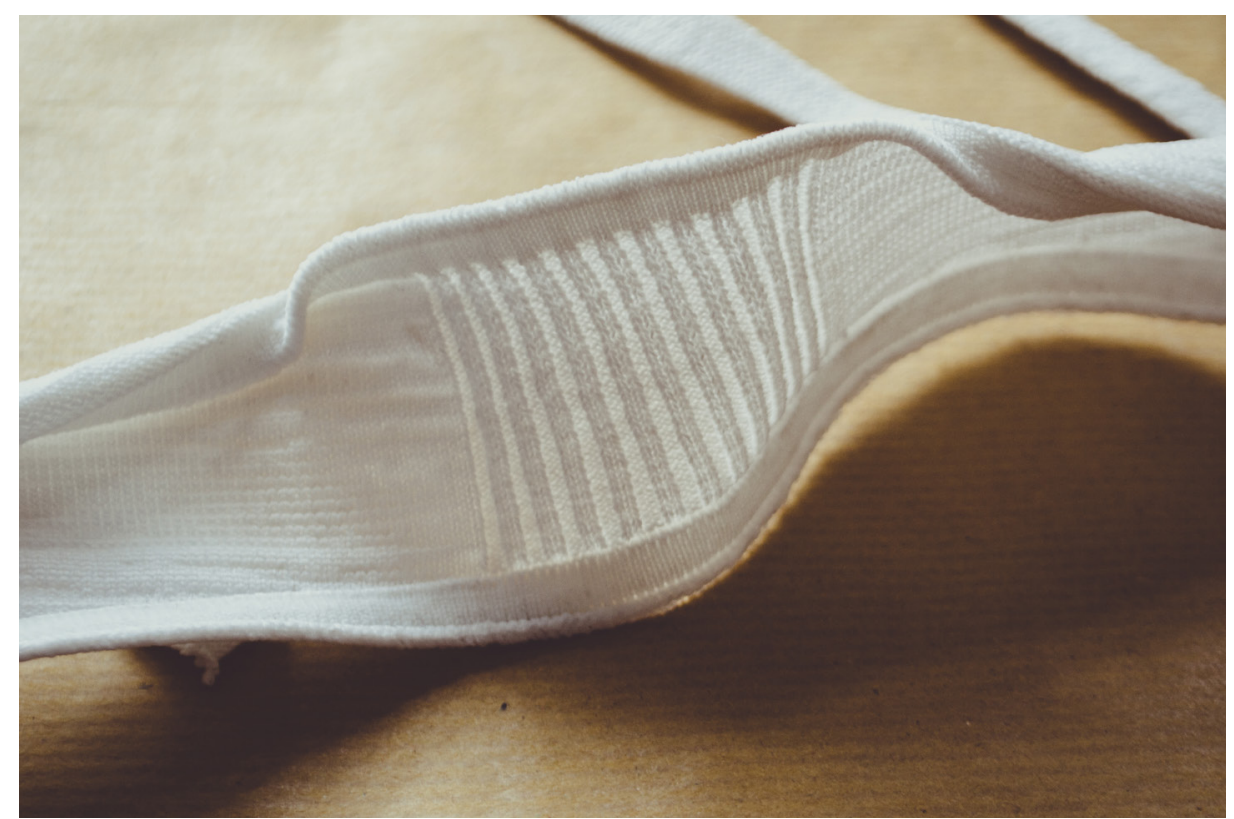

\section{Yarn and structure development}

\section{Series IV}

At this time lycra had been knitted throughout the whole garment, the band, cups and straps included. The lycra added some stretch as well as created a thicker denser fabric feel. It also helped smoothen the polyester band to lay flat. The polyester was very difficult to knit by itself. Due to its fibrous nature, the thin threads kept getting knotted or intertwined in each other when being knit. A thinner strap was added to the middle of the cup extending straight into the main shoulder strap. This was to help hold the cup shape so that it would be less likely to curl. The straps were initially developed with a simple $1 \mathrm{x} 1 \mathrm{rib}$, but that proved to be way too stretchy, so in this iteration the strap knit structure was replaced with an interlock structure which limited the overall stretch in both directions. Additionally, the bottom hem had been extended vertically, which would help with a more supportive shape. Additionally, the first development of including specific knit structure was implemented in this series of iterations. Knit structure changes the amount being knitted, for instance, a mis-knit stitching will offer a tighter, less stretch knitting compared to a plain stitch. When these two stitching structures are knitted there are noticeable size differences. Therefore, there must be care when adding structure, and change in overall shape must be considered. Through literature, it has been noted that the side panels of the bra should be quite low in stretch so that it can give a tighter and supportive feel, thus a mis-knit stitch was incorporated on both sides of the cups. As for the back, since this bra design has a front closure design, the back can be released of the pressure that the hooks and seams usually give off. The back panel was given a $2 \times 2$ rib structure for comfort and a great amount of stretch. 


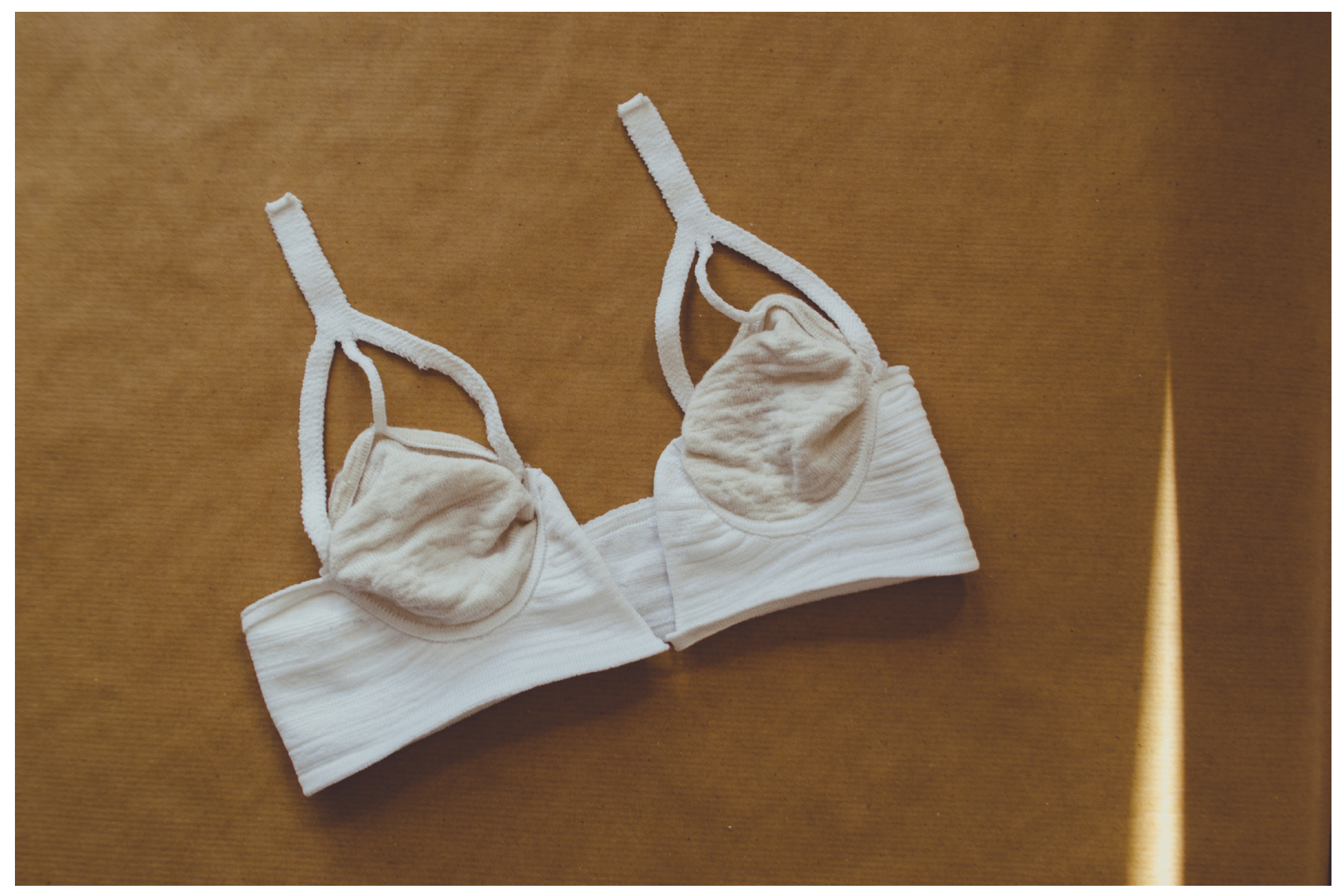




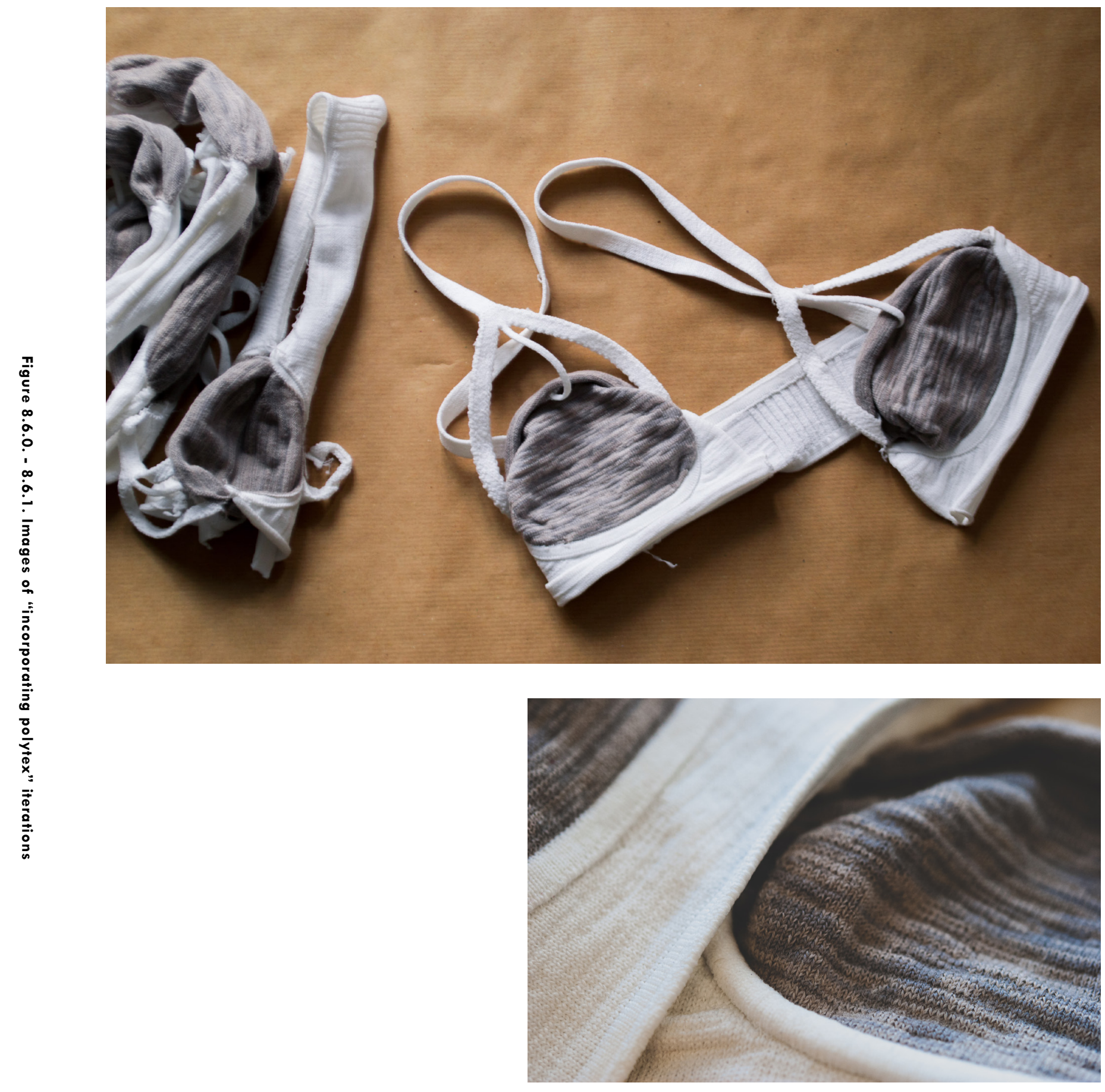

$\vec{\infty}$ 


\section{Incorporating pemotex}

\section{Series VI}

The iterations in this series involved the introduction of a new yarn, pemotex. Pemotex is a synthetic yarn which has an interesting property of becoming completely stretchless and hardened after being steamed. The pemotex was applied to underwire section. This could potentially help reinnovate how a bra should perform and support the breasts, and challenge the design of a traditionally wired bra by offering support without the discomfort of a hard underwire.

Experiments were first conducted to test a variety of knitting techniques in conjunction with the pemotex to see how the yarn performs and how it can best be used for the underwire placement. Tubular knitting structure was tested with a $1 / 2$ stitch in the back panel, $1 / 3$ stitch in the back panel, and a $1 / 4$ stitch in the back panel. By stitching a normal stitch in the front bed and less stitches in the back it would hold the back needles and consequently create a rounded effect in the front bed, the result looks like a hem with slight elevation which can be used to mimic the underwire section. The true ripple effect technique involves the back bed holding its stitches and the front bed stitching a plain stitch. This creates a rounded elevation effect as well but it is less structured and more drooped. These following tests resulted in multiple underwire possibilities for the final design:

- No underwire, just a seam to line the outline

- Flexible tubular slot with no external underwire

- Non-flexible hard 1/4 tubular slot with addition of external underwire

\section{Merino cups}

\section{Series $\vee$}

Merino yarn for the cups was an experimented yarn choice that was continued through the design process. Merino with lycra threaded simultaneously thickened the cup which offered better shape as well as stretch which is desirable for movement and comfort. The properties of fine merino include breathability, durability and long stability. Merino will also soften over time allowing for ease of wear. For this research, materials were considered that offered a calming feel, alternative to many over embellished bras with excess of lace and hard seamed structures that create a stiff and high pressure wear. Instead the knitting iterations were designed to be a lowpressure bra that will be able to move and grow with the wearer. In this series, the holes and loose stitching were resolved by fixing the layoff stitching. As mentioned before it was predicted that the loose stitches and holes were caused by the needle pulling the thread. Additionally, the straps were measured and then designed to be knitted at its full length.

- Non-flexible hard 1/4 tubular slot acting as the underwire itself

After the preceding tests, the close knit structure was using pemotex with a tubular knitting with $1 / 4$ less stitching in the back bed. The chosen measurements were 8 stitches in the front bed and 2 in the back bed. This allowed for a nice three-dimensional and rounded feature that was steamed solid. This iteration could be compared to the iteration right before it, though in both iterations the pemotex was used, there is a clear difference between the pintuck and the tubular stitch structure. The pemotex tubular structure gives structure to the underlining of the cup area, however not enough density to be used as an alternative to the underwire. Since it is still tubular, an additional wire could be potentially threading through the slot. On the other hand, the pemotex being used in the pin tuck iteration shows a successful application in creating an alternative to an underwire due to its density and $3 \mathrm{D}$ form which mimics the structure on the underwire, and $3 \mathrm{D}$ form gives a potentially greater shape than a flat underline. 


\section{PARTICIPANT FITTING}
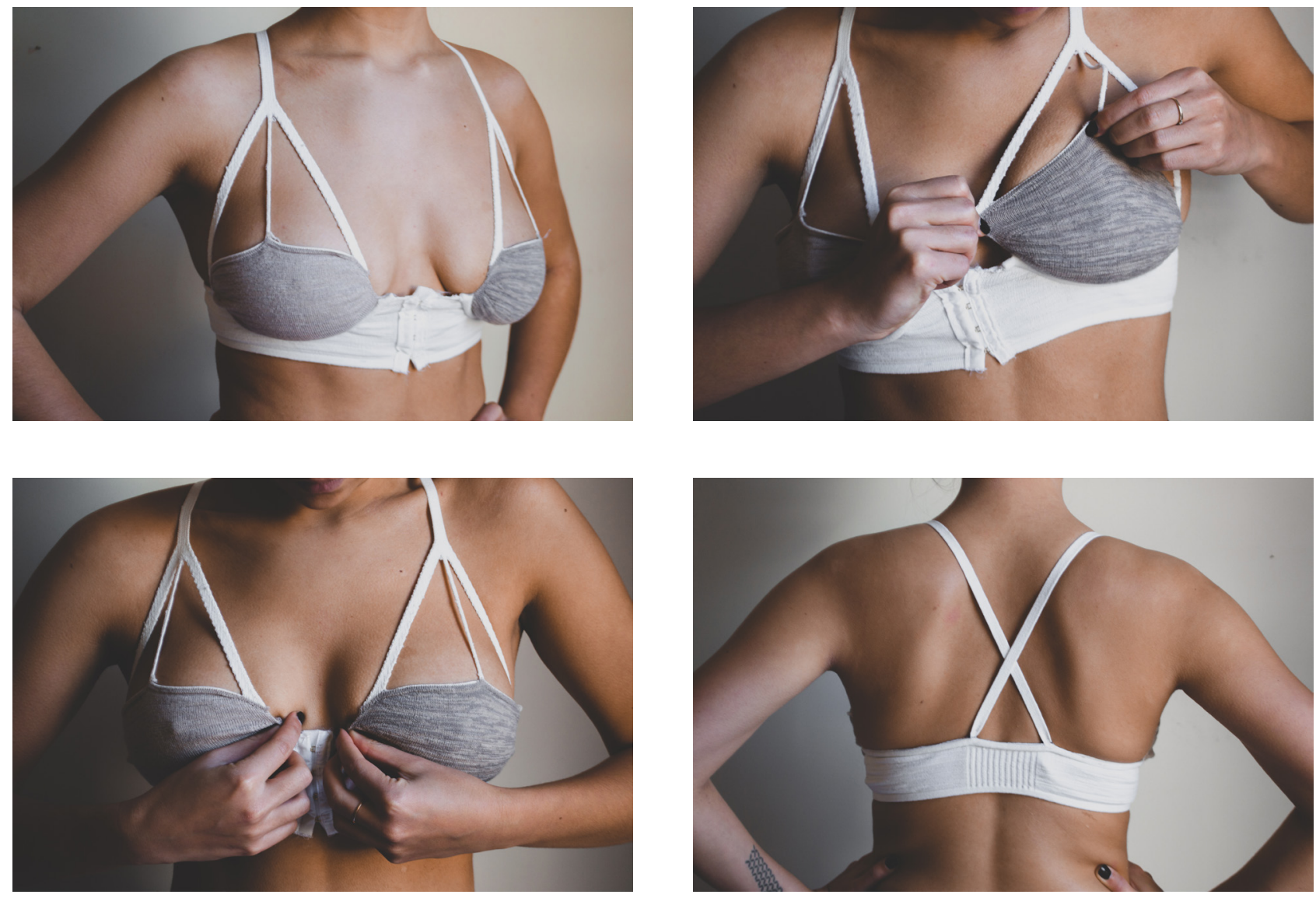

$\overrightarrow{0}$ 

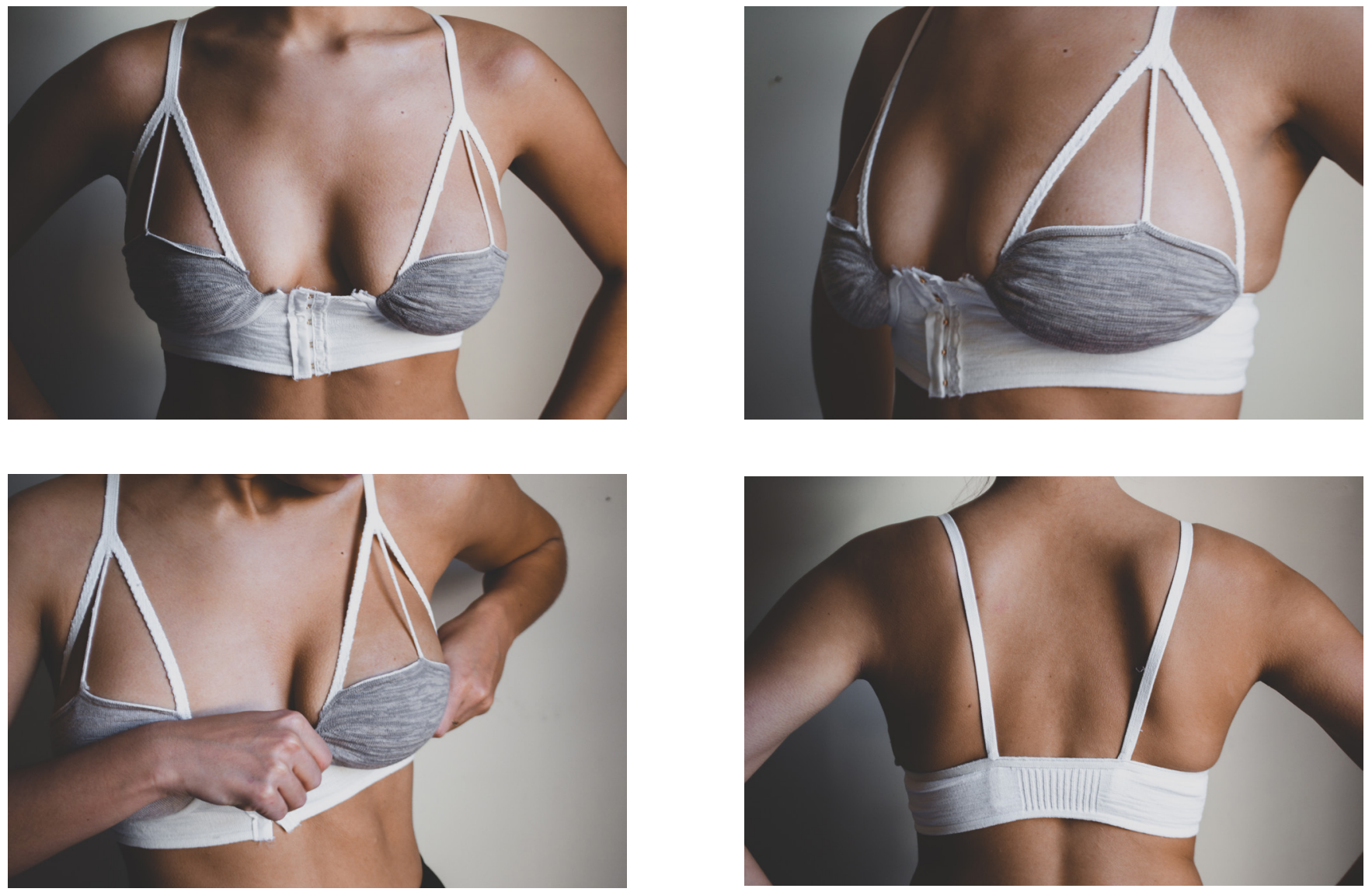

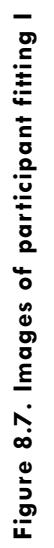


Two knitted bra prototypes were worn and tested by the participant. The two bras selected were the final two bras knitted in the iterative process, these bras were the most developed in terms of yarn choice, structure implemented and shape desired. Both bras were practically identical in design and both contained the polytex underwire. The only difference was that one bra contained a ridged underwire, with a polytex bottom hem and four front hooks, and the other with a tubular knit underwire with a polyester bottom hem and five front hooks. The main limitation of these bra prototypes were that they were developed through a research through design process to which the final design was based upon the preceding iterations, in other words, the bra prototype design was based upon experimentation of its past iterations. Therefore the bra prototypes were not made-to-measure and thus not measured or sized to the participant testing them. This testing session was not to determine a good fit, but more to offer insight of the participant's initial thoughts and suggestions of a non-traditional 3D knitted bra. An assessment of fit is addressed in the following two chapters, Chapter 9 and 10, where the measurements from the $3 \mathrm{D}$ scanned data are implemented.

The participant was asked to try on each bra and participated in a semi-structured interview (Martin \& Hanington, 2012, p. 102) to analyse the design. For both bras, it was immediately clear to the participant that the bra did not fit well. She specifically had a problem with the cups being "too far apart and too shallow", and did not cover the breasts enough length-wise. There was an overall lack of lift and support in the cup region. The participant expressed overall positivity when asked about the front-closure design, where the hooks were placed in the front of the bra, as opposed to more commonly in the back. She especially enjoyed the design of the gap between the cups and front band. Additionally, the materials, the yarn choice, was deemed a favorable aspect. However, she noted that it was too difficult to critique the way the material would influence the shape of the breasts since the bra was not the right fit. 


\section{F E E D B A C K B Y S E C T I O N}

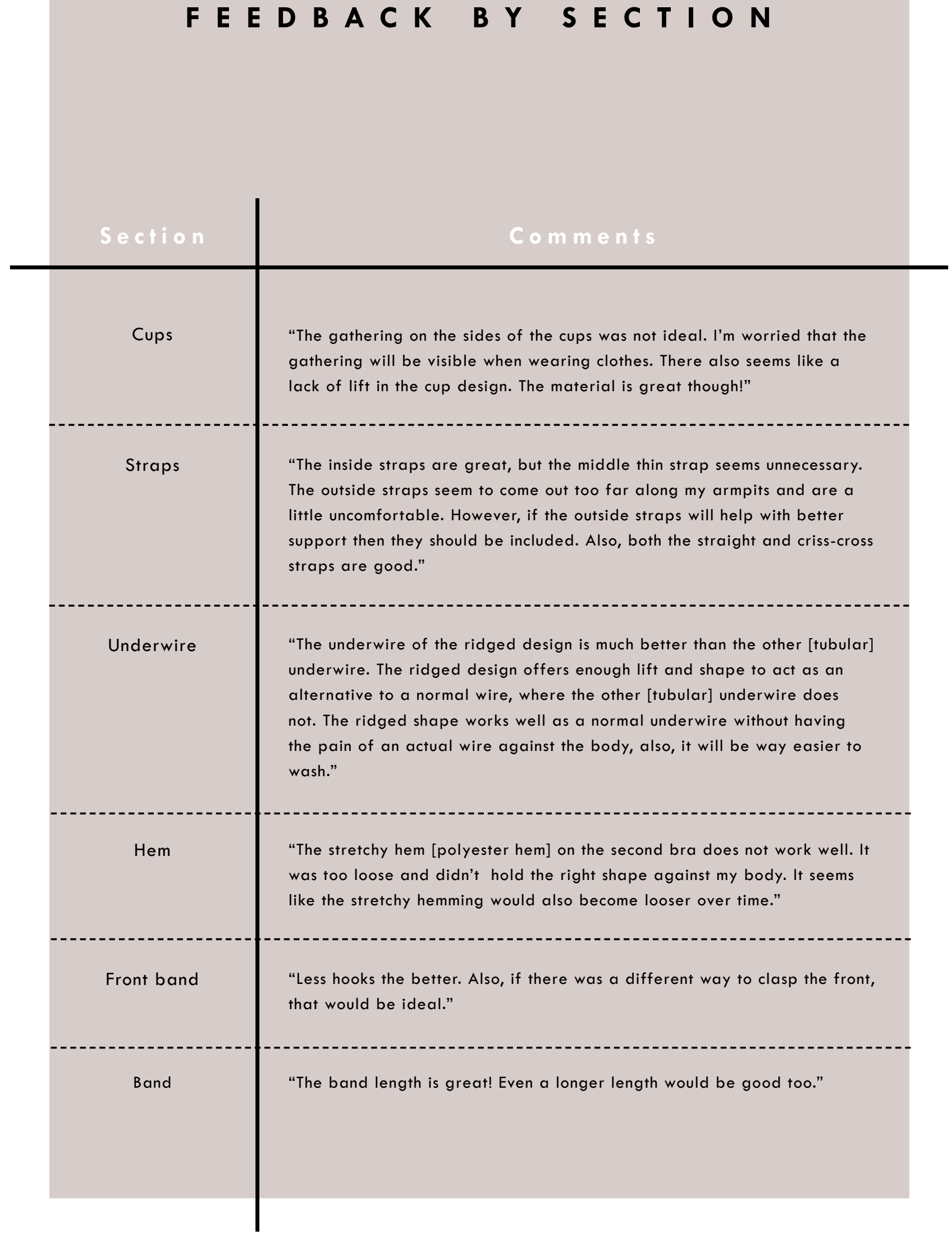




$$
\begin{aligned}
& M A \\
& \triangle A M \\
& M A D
\end{aligned}
$$




$$
\begin{aligned}
& M \\
& M \\
& M
\end{aligned}
$$




\section{KNITTING BASED ON \\ FEEDBACK}

\section{Vertical cup seam}

\section{Series VII}

Many suggestions were received from participant feedback, majority were small design preferences between the two prototypes tested, but the most crucial point of concern was the structural support of the cups. The participants noted that there was a lack of lift and shaping the cups offered, explaining that the knitting structure of gathering on the sides was not favorable. It has been noted that for non-padded soft bras seams are an important feature to maintain structure and form a desired breast shape. Therefore, for the following iterations the addition of a cup seam is explored to respond the lack of cup shape. However since the seam is vertical, creating a protruding feature is not as easy as simply add a line of structure like it would be on a horizontal line. Since the knitting machine builds up the garment horizontally, to create a seam that protrudes vertically will require some experimentation. Experiments were knitted on a basic square piece, with four lines of structure: $1 / 2$ tuck in the front; $1 / 2$ tuck in the back; $1 \mathrm{x} 1$ rib; plain, all knit. These experiments attempted to imitate structure of a vertical seam, however were limited by their effectiveness to stand out. Another experiment to develop a ridge for the cup seam was created using a type of lace stitch but covering up the holes created with a kickback. This experiment was quite successful in that it not only provided the necessary protruding form of seam, but it also gathered in the edges of the fabric uplifting and created a dome/3D effect which is highly favorable.

Rayon was introduced as a yarn to replace polyester to explore alternative yarn types. Though a synthetic fiber, the following iterations hoped to explore the silky aesthetic rayon develops when being knitted. Three bra piece iterations were knitted with the new yarn and the ridge for a vertical seam in the cup region. Though none of the iterations knitted successfully, through problems with machine knitting the rayon and pemotex, the ridge in the cup proved to be a very successful method of providing a full 3D form, and hopes to be better suited for breast shape in the bra. Since the rayon and pemotex combinations were causing unsuccessful knitted pieces, the final iterations in this series replaced the rayon with merino/lycra thinking that this yarn change would allow for a cleaner knit. Though the piece was able to knit there still remained issues, such as the cup not seamlessly knitting into the front band. These problems mirrored earlier issues with holes appearing in the cup to band region in the knitting iteration series II and III. 

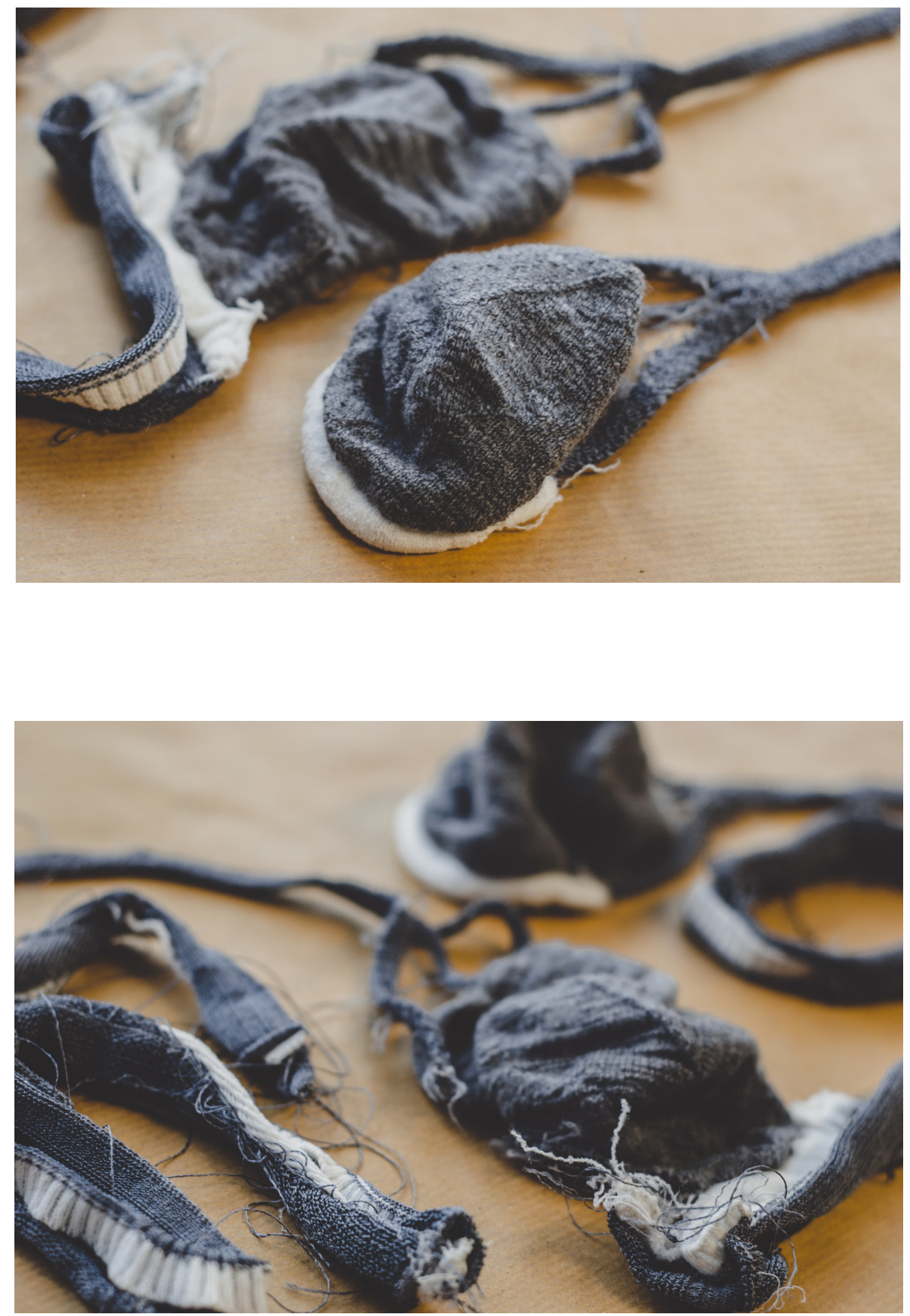

in 
CHAPTER 08

BRA DESIGN DEVELOPMENT:

3D KNITTING EXPLORATION

\section{DISCUSSION}


The resulting bra prototypes designed as a result of the iterative process hoped to use the many unique properties of the 3D knitting machine. The use of a $3 \mathrm{D}$ knitter in bra design is not a new technique, however, the majority of commercial bras using the 3D knitter are structureless, based on 2D patterns, knitted with rough nylon elastane, and designed as bralet or pull over, slip on bras. They have been noted to be comfortable, however are limited in adequate support as well as forming the desired breast shape for an everyday bra. The bra prototypes developed in this chapter hoped to address the lack of threedimensional shape in current 3D knitted bras, as well as create a range of knit and yarn experiments to address support, comfort and pressure concerns.

Developing prototypes was quite an extensive process that was highly dependent on trial and error. Though there was a clear design idea at the beginning of the process, in order to achieve it, a series of iterative prototypes were needed. Each iteration represented a development towards the final piece. Each adjustment or alteration needed to be knitted in order to adequately test its ability to succeed. Each change in shape, knit structure, yarn type and measurement will yield quite different knitting results, therefore, even the slightest and smallest changes must be knitted as a physical output. However, once a final prototype bra has been developed, and a final design structure decided, the nature of $3 \mathrm{D}$ knitting insures that replicating the exact garment will be easily achieved for each individual. Additionally, changes of the design are capable through editing the digital knitting software so that a new outcome does not have to be developed from a blank design.

Through the first prototypes developed (series I to VI), significant strides were made in terms of developing a seamlessly linked, cup to band to underwire design, where specific knitting structures were explored to offer alternatives to traditional bra design, as well as help with overall comfort and a low-pressure design (the softness and flexibility of merino/lycra and the firmness of pemotex to provide wireless underwire while maintaining desired shape). However, the largest limitation remained the cup design. To this point the cup is designed based on the gathering structure in the IBP and OBP corners, this gathering offers slight $3 \mathrm{D}$ forming which is then stretched when worn. Through testing with the participant it was clear that this method of forming the cup design based on gathering the sides was not sufficient in $3 \mathrm{D}$ forming nor could provide adequate shaping. An alternative method for creating a more $3 \mathrm{D}$ form that could better shape and support the breasts was necessary. The second prototypes (series VII), though not knitted at a level which could be worn and tested, demonstrated the ability to knit an improved 3D knitted and 3D shaped cup.
In terms of measurement, there are aspects of the breasts and body that are vital to acknowledge when considering bra fit (these are addressed in the previous chapters). Though these many measurements remain tremendously important to bra design, in this chapter, and point in the design process, understanding the $3 \mathrm{D}$ knitter's properties in relation to a bra design development took priority over measurement and sizing. After these processes were explored and a successful overall bra design was developed, then the specific measurements obtained from breast data were applied, as discussed in the succeeding chapter. 



$$
\text { C H A P TER } 09
$$

BRA DESIGN DEVELOPMENT:

UTILIZATION OF MEASUREMENTS 
This chapter brings together point measurements and design parameters to develop bra prototypes that follow the whole system principle, proposed in Chapter 03.

Two bra prototypes, Scan to Knit and Scan to Sew, were developed using two different manufacturing techniques but with the same measuring system, as described in Chapter 07. The first bra, Scan to Knit, is a continuation of the iterative process, explored in Chapter 08. It follows the previously developed knitting techniques and includes adjustments based on participant's feedback. However, Scan to Knit also incorporates accurate measurements of the participant into the knitting software. Bra, Scan to Sew, is produced by the traditional cut and sew method. This prototype is also sized dependent on the measuring system. The scanned 3D mesh model of a particular participant (henceforth Participant-2) was used for the development of both these prototypes. Participant-2 was chosen due to her smooth and error free scan data, as well as her readily availability during the design and manufacturing process. The overall aim of this chapter is to produce two different bra prototypes with different manufacturing methods, but based on the same participant's 3D scan and measurement data. 


\section{MO D I F I E D C R I T E R I A}

The initial criteria for manufacturing process of a physical bra (explained in Chapter 8 ) were examined and adjustments were made to make improvements for the final bra design outcomes. The initial criteria for the 3D scanning process, as discussed in Chapter 7 , did not require any modifications. The initial manufacturing criteria were specific to the $3 D$ knitting process but in order to include assessment of the cut and sew method bra prototype, a new set of criteria were required to address both techniques.

Distribution of pressure

Longline design.

The band must be extended long enough to aid in overall support.

Cup should lift and encase the breasts.

Cup form

The cups must be designed using knitting or sewing techniques to support the breasts appropriately.

Cup form

Vertical seams should be utilized in the cup design.

Vertical seams must be incorporated in most manufacturing processes to help shape the cups. 


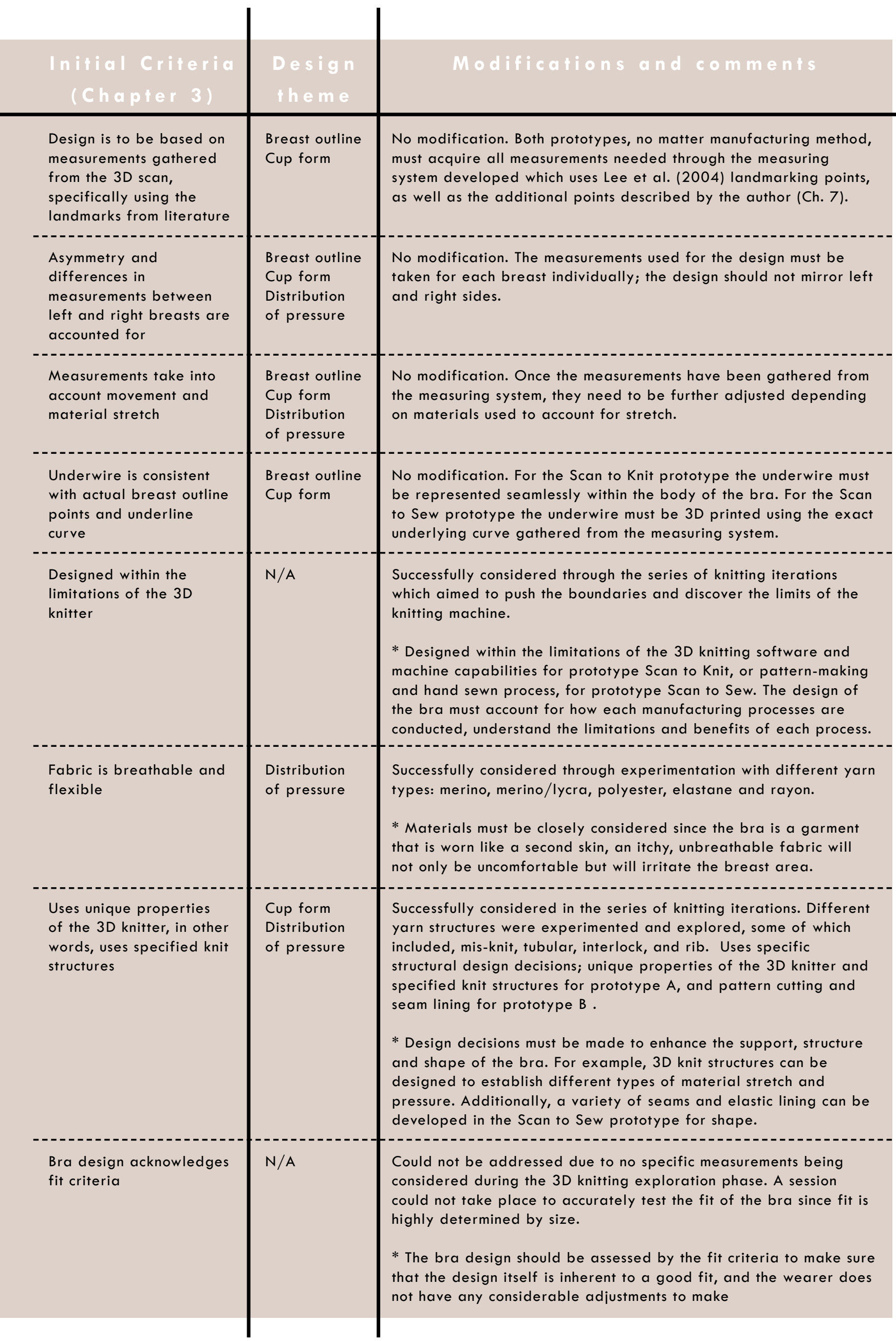




\section{SCAN TO KNIT}

The knitting exploration of Chapter 08 helped establish a bra design that incorporated a seamlessly knitted cup, front band, side panel, back band, and straps. Additionally the series of iterations led to an exploration of different knit structures and yarn types, resulting in plain knit for the cups and front band, mis-knit for the side panel, rib for the back band and bottom hem, and tubular for the straps. The cup design was further developed after participant-2's feedback, which suggested a lack of lift and desired breast shape. A vertical seam was therefore applied to the cup to enhance the structure, as well as simultaneously knitting a more 3D shaped form. This section discusses the continuation of all these components, while implementing the appropriate measurements gathered from the Participant-2's digitally scanned 3D mesh model.

The measurements needed for the Scan to Knit prototype were gathered through the parametric measuring system. The necessary measurements and associated landmarking points used are described in the table to the right. These measurements were selected based on how the design is drafted on the Shima Seiki digital knit software. 


\section{S C A N T O K N I T \\ M E A S U R E M E N T S}

Cup distance vertically to show knit length

BBP to UBPA (right and left, $R$ \& L)

Front band distance horizontally, from inner

$\operatorname{IBP}(L)$ to IBP (R)

breast underline point

Front band distance vertically, from top of

$F C P$ to $F B B P$

the front band to the bottom

Lowest underline point to bottom of the

band vertically

$B B P$ to $F B S P(R \& L)$

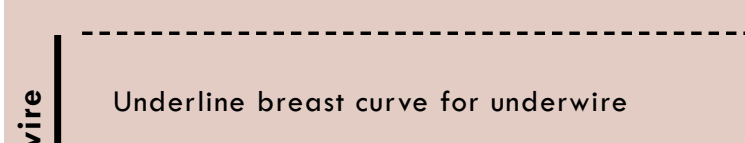

$O B P$ to $B B P$ to $I B P(R \& L)$

Top of breast underline distance horizontally

$I B P$ to $O B P(R \& L)$

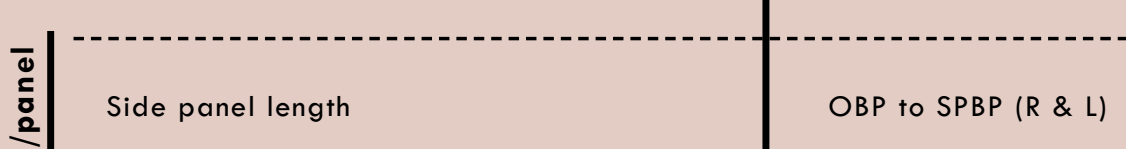

등

Top of band curve length

$F B B P$ to $S P B P(L)$ to $B B B P$ to $\operatorname{SPBP}(R)$ 


\section{SCAN TO KNIT \\ MEASUREMENTS FOR \\ DIGITAL KNITTING}




\section{U P}

LENGTH: BBP \& UBPA (R \& L $)$
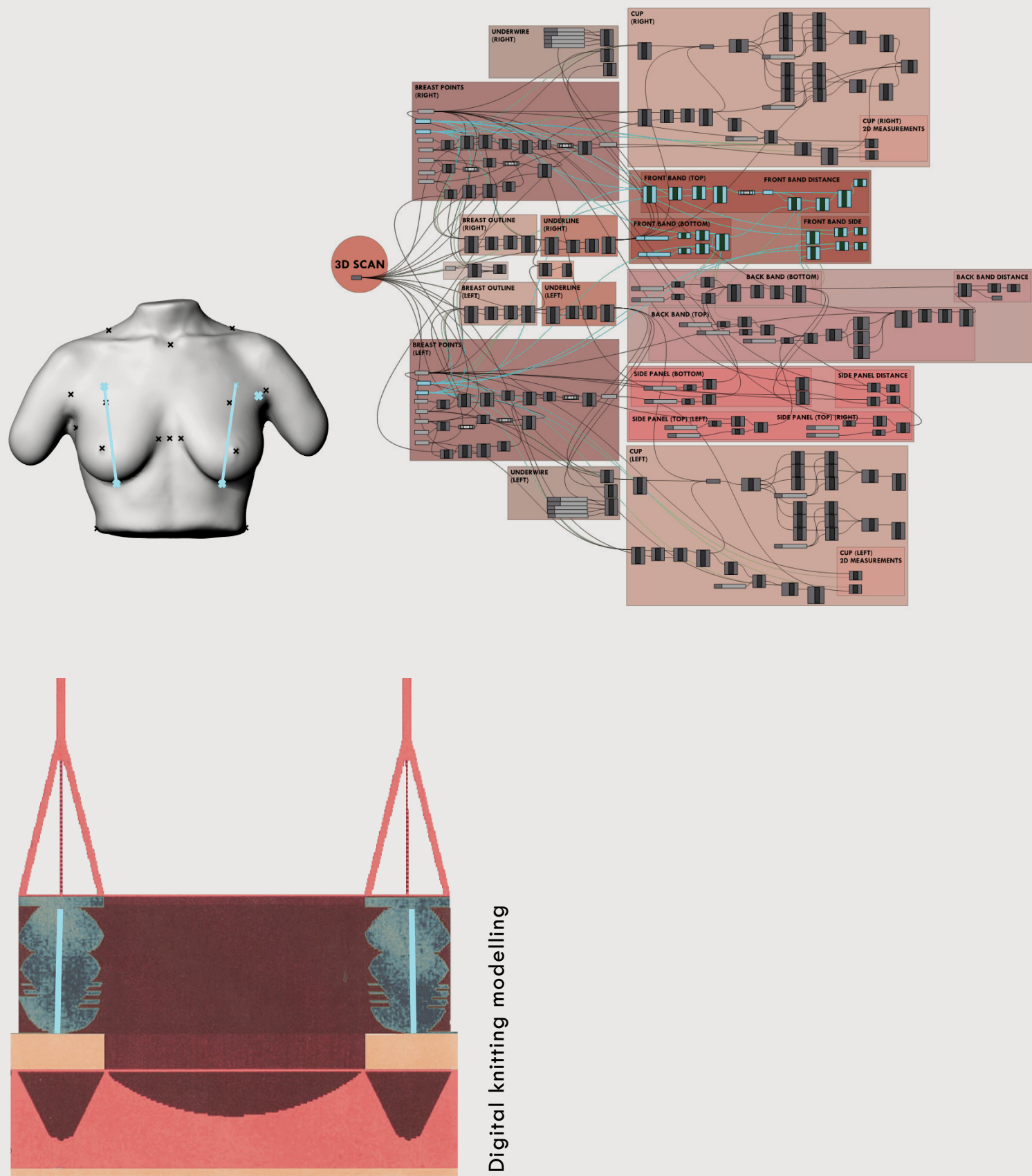

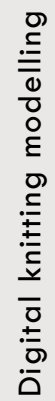




\section{F R O N T B A N D}

$T O P: I B P(R) \& I B P(L)$

LENGTH: FCP \& FBBP

SIDES: $B B P$ \& FBSP $(R \& L)$
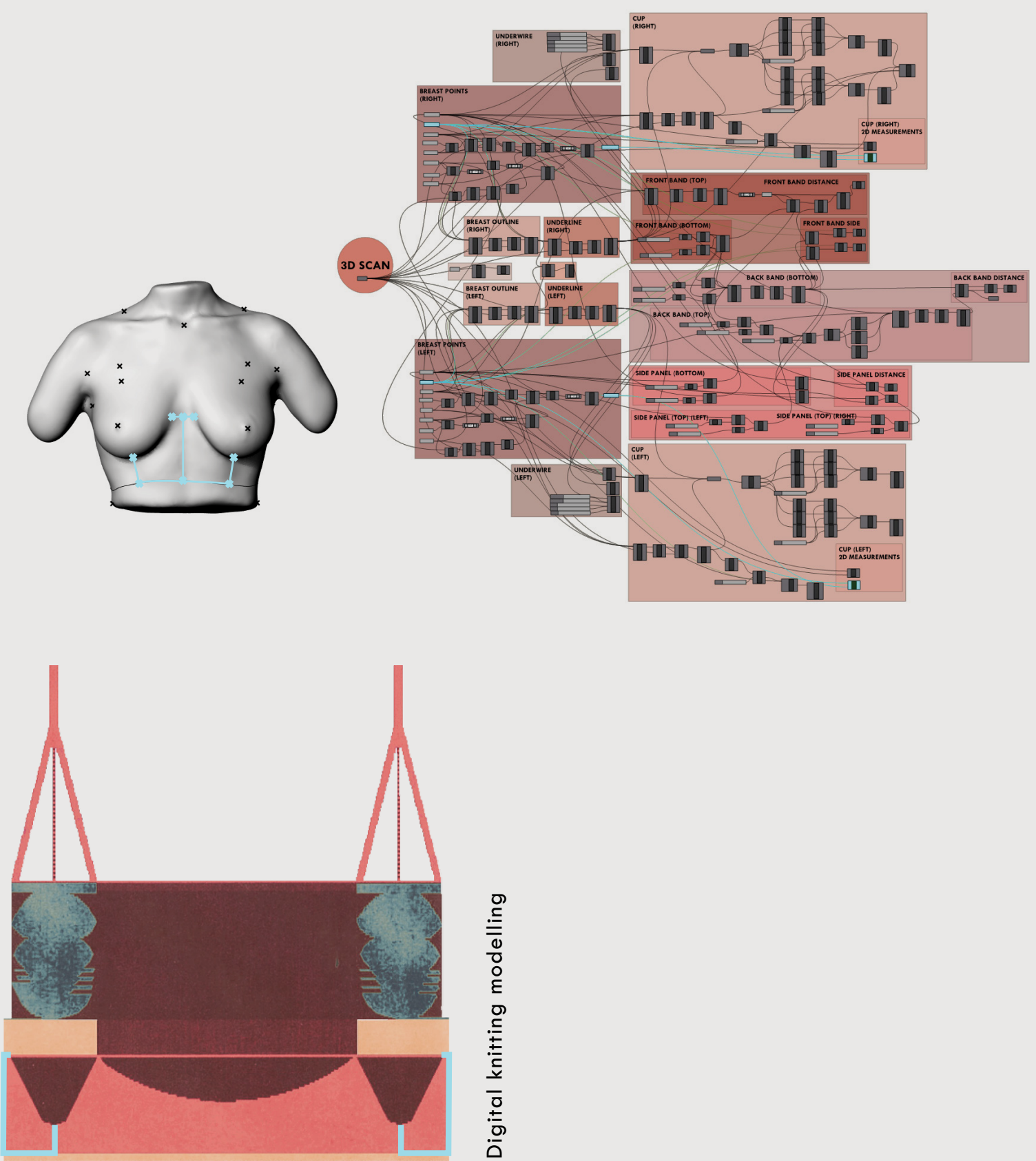

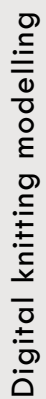




\section{U N D E R L I N E / U N D E R W I R E}

UNDERLINE CURVE: OBP, BBP \& IBP (R \& L)

WIDTH: IBP \& OBP (R \& L )
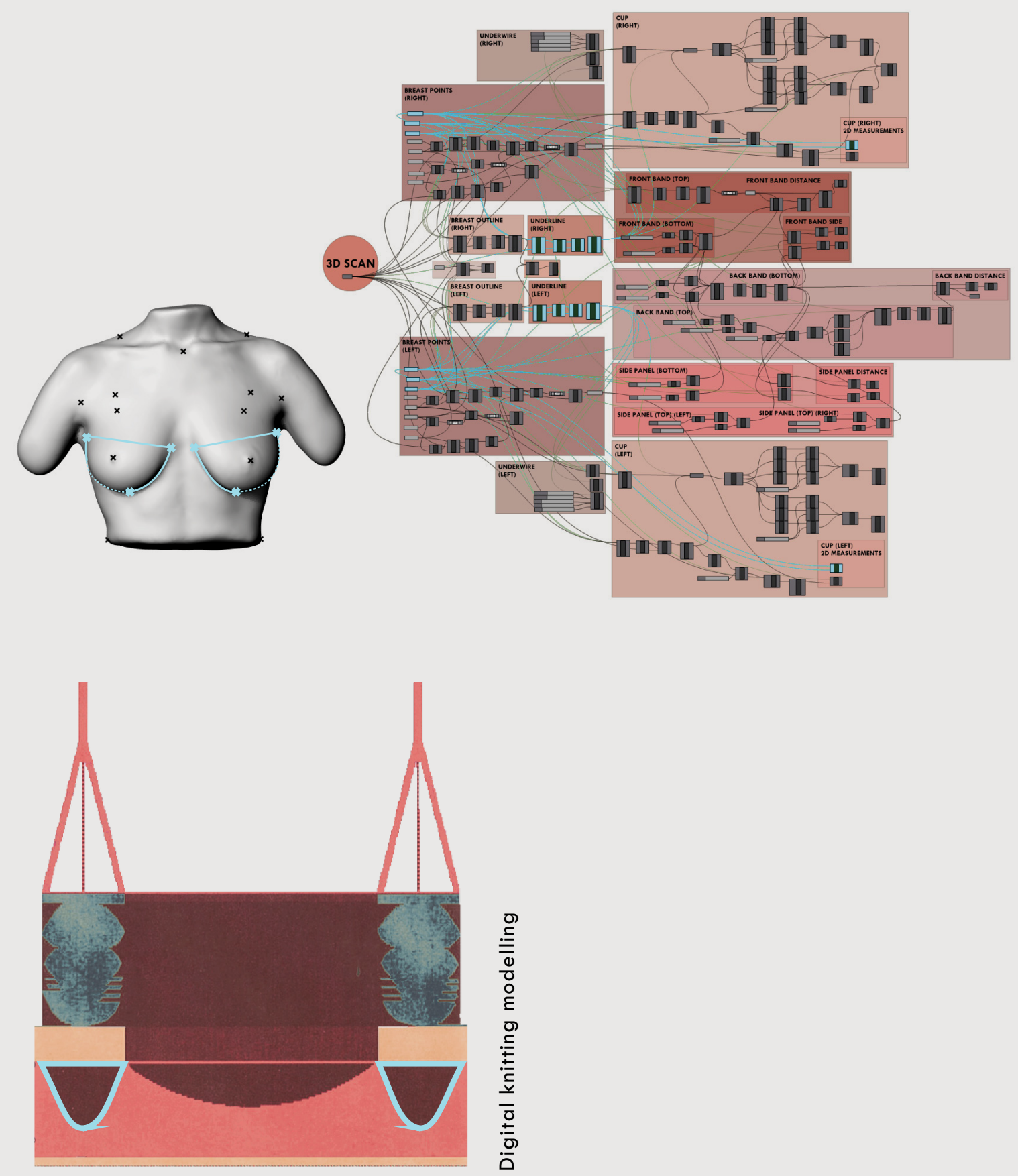

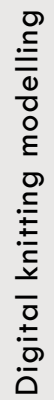




\section{S I D E P A N E L}

LENGTH: OBP \& SPBP (R \& L $)$
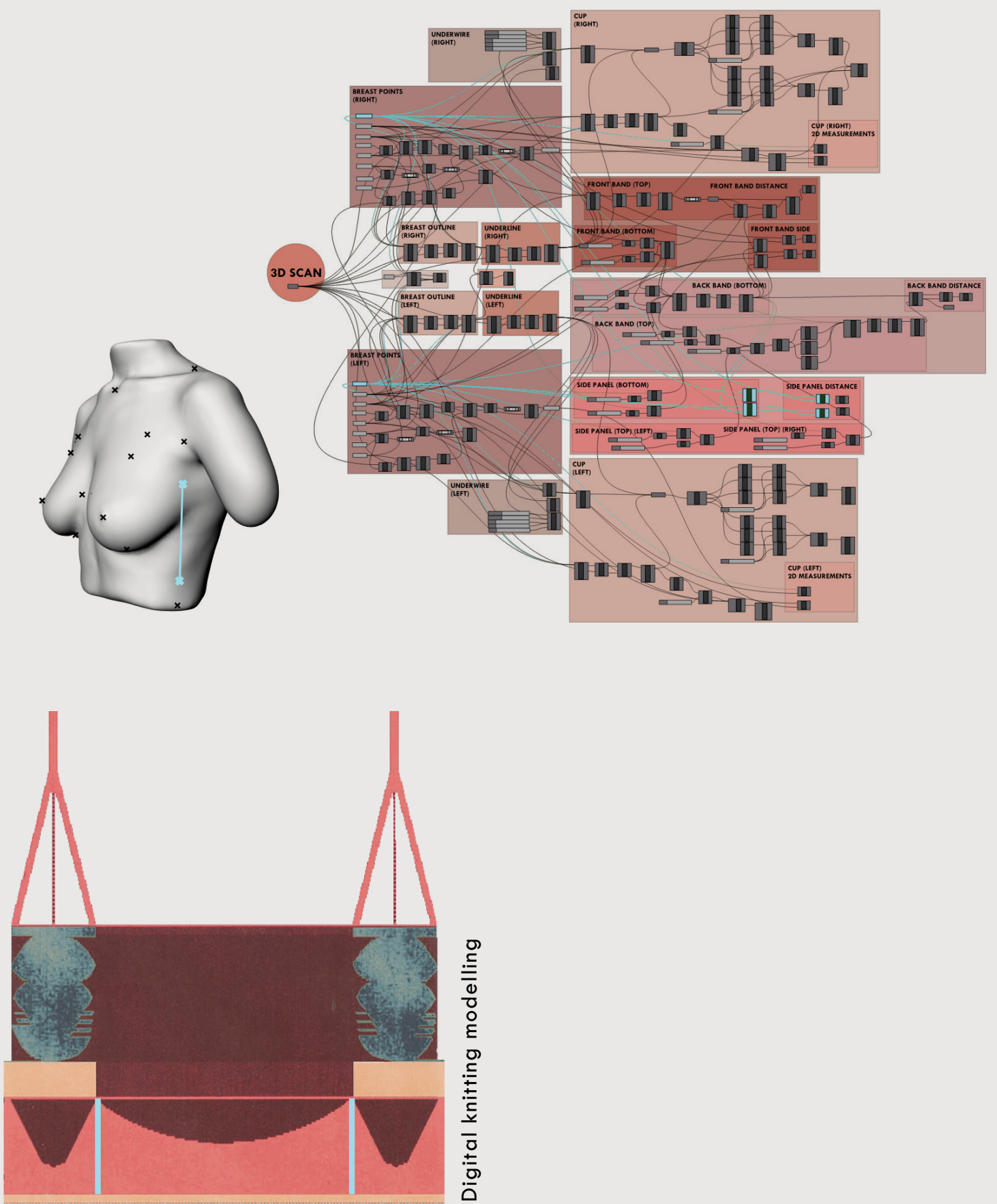


\section{B A C K B A N D}

TOP: OBP (R \& L), SPTP (R \& L), \& BBTP

$B O T T O M: F B B P, S P B P(R \& L), \& B B B P$

LENGTH: BBTP \& BBBP
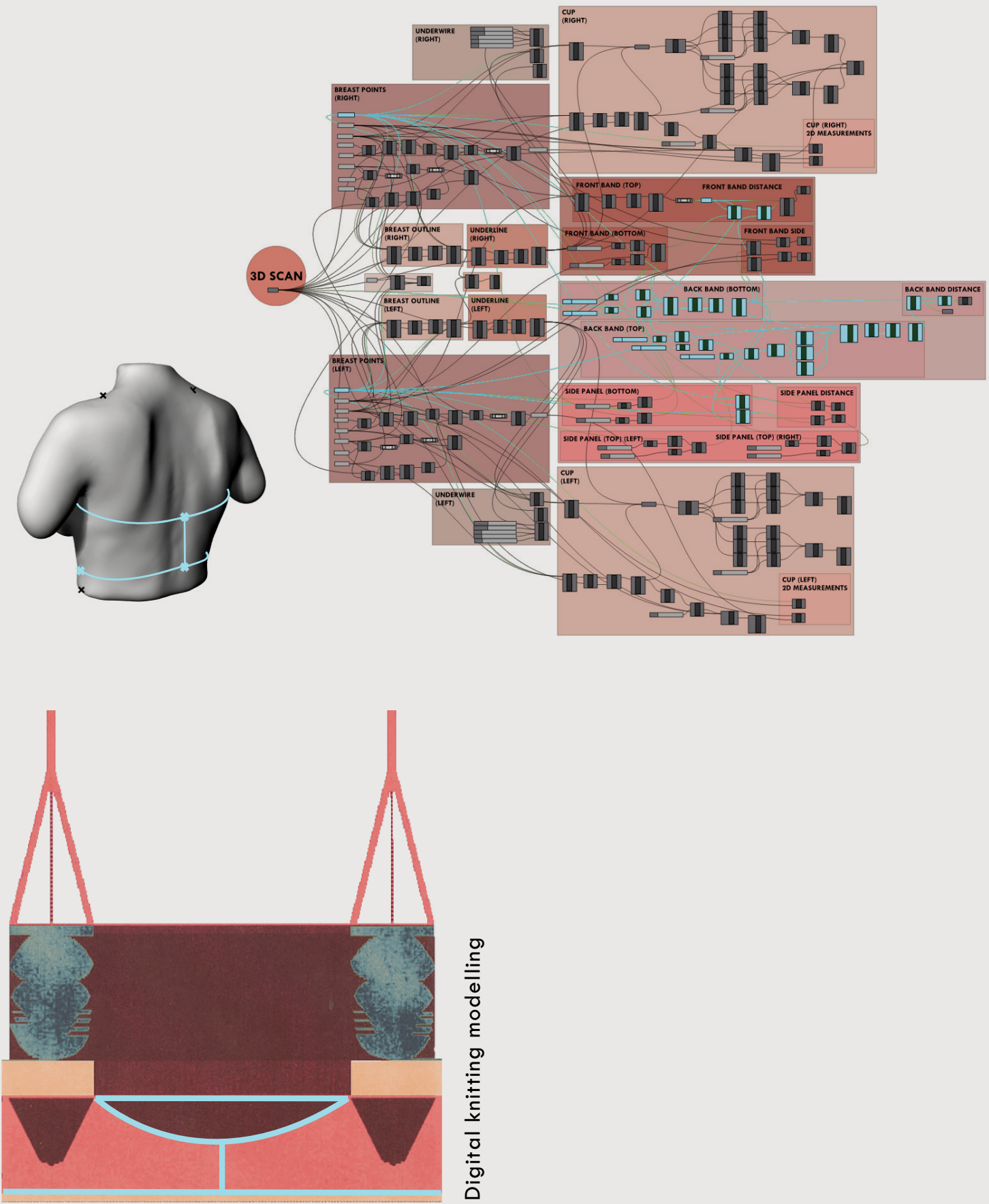


\section{Application}

In order to utilize the measurements gathered from Chapter 07, the latest prototype developed from Chapter 08 had to be analysed and manually assessed. This step was needed to account for how the knitted piece behaved after completion. In other words, since sizing can be altered by yarn choice and knit structure, this physical piece needed to be measured to act as reference when accounting for the new desired measurements taken from the participant's scan data.

All measurements were able to successfully translate into the digital knitting software excluding the breast underline curve measurement which is used to define the knitted underwire, and the connecting side panel measurement which connects to the OBP point of the underline curve, underwire curve. The breast underline curve is the curve that not only dictates the angle, shape and length of the underwire but also the distance between the OBP point and the bottom of the band (the measurement used for the side panel). A curve was able to be made in the software, but not at the correct length or angle needed. The inner part of the curve (connecting to the IBP), in the front band, was successfully knit at the correct measure, but the outer part (the part that connects to the side panel and OBP) was not successful. To change the shape of this outer part of the curve would affect the knitting developments of the cup form. At present, the underwire curve is a symmetrical U-shape at which the cup shape knits within. Future research, and knitting exploration, would need to consider how to angle the underwire shape to account for asymmetry in its nature.

In terms of materiality and structure, the prototype continued the same merino/lycra combination and used several of the same successfully knitted structures as previous iterations. Minor changes were made in the knit structure to include a rib bind off at the top of the band to address unwanted "curling". 

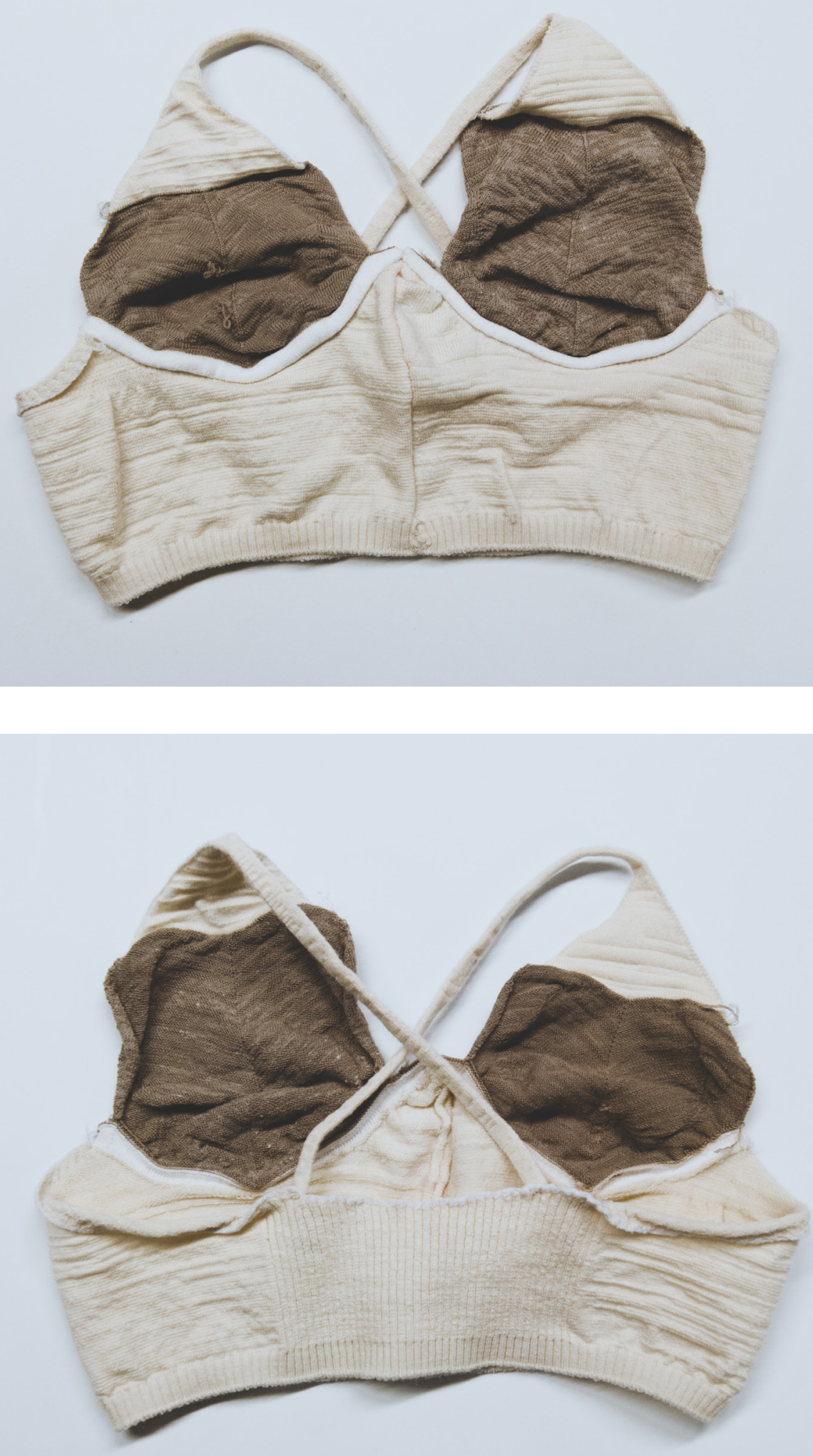


\section{S C A N T O S E W \\ ME A S U R E M N T S}

Nipple to end of underwire at side, (L) and

(R)

$B P$ to IBP (R \& L)

Nipple to end of underwire at centre, (L) and $(R)$

$B P$ to $O B P(R \& L)$

Nipple to base of breast, (L) and (R)

$B P$ to $B B P(R \& L)$

Distance between underwires at centre front

IBP $(L)$ to IBP $(R)$

Depth of longline from centre front down

$F C P$ to $F B B P$

Depth of longline from base of breast down

$B B P$ to $F B S P(R \& L)$

Depth of longline from side seam down

$O B P$ to $S P B P(R \& L)$

๖ำ

Back panel length

BBTP to BBBP

Centre front to centre back bust measurement for (L) and (R) - the widest point of bust, line usually runs through nipple point

Centre front to centre back ribcage measurement for (L) and (R) - directly beneath the breast, where breast meets ribcage.

Bust curve measurement around torso, including $O B P, B P, \& I B P$ 
The following section discusses an experimentation of the traditional sewing manufacturing method, Scan to Sew, to compare the different qualities given compared to the Scan to Knit method previously explored. This comparison was particularly important regarding the limitations found within the $3 \mathrm{D}$ knitting manufacturing process. In order to develop a bra prototype in this context, the expertise of a research assistant from Massey University, School of Fashion, was acquired.

Unlike the Scan to Knit measurements, which were gathered from the digital parametric measuring system, manually adapted for material consideration, and then input digitally into the Shima Seiki digital knit software, the Scan to Sew bra design utilized the measurements manually. A list of measurements, in the table below, is based on fashion design literature for bra design. These measurements were obtained digitally through the parametric measuring system, but then drafted manually using traditional pattern-making techniques (this method of design drafting is discussed in Chapter 02, Section IV). The manually drawn patterns were formulated and cut into the designated pieces. These pieces were then used to compose the prototype. 


\section{SCAN TO SEW \\ MEASUREMENTS FOR \\ CUT \& SEW}




\section{U P}

BUST CURVE 1: $B P$ \& IBP (R \& $L)$

$B U S T$ CURVE 2: $B P$ \& OBP $(R \& L)$

$B U S T$ CURVE 3: $B P$ \& $B B P(R \& L)$
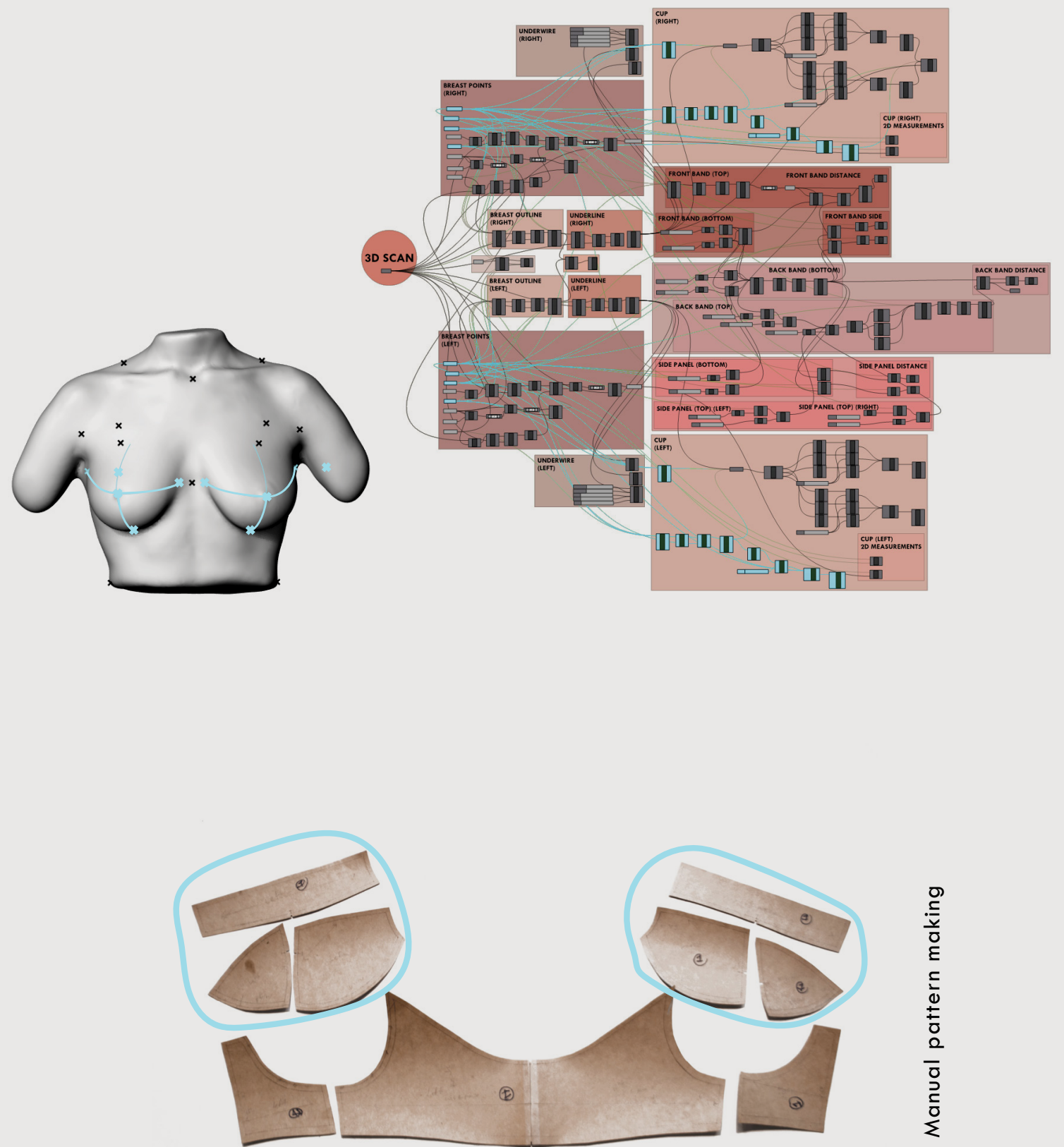


\section{F R O N T B A N D}

$T O P: I B P(R \& L)$

LENGTH: FCP \& FBBP

SIDES: $B B P$ \& FBSP $(R \& L)$
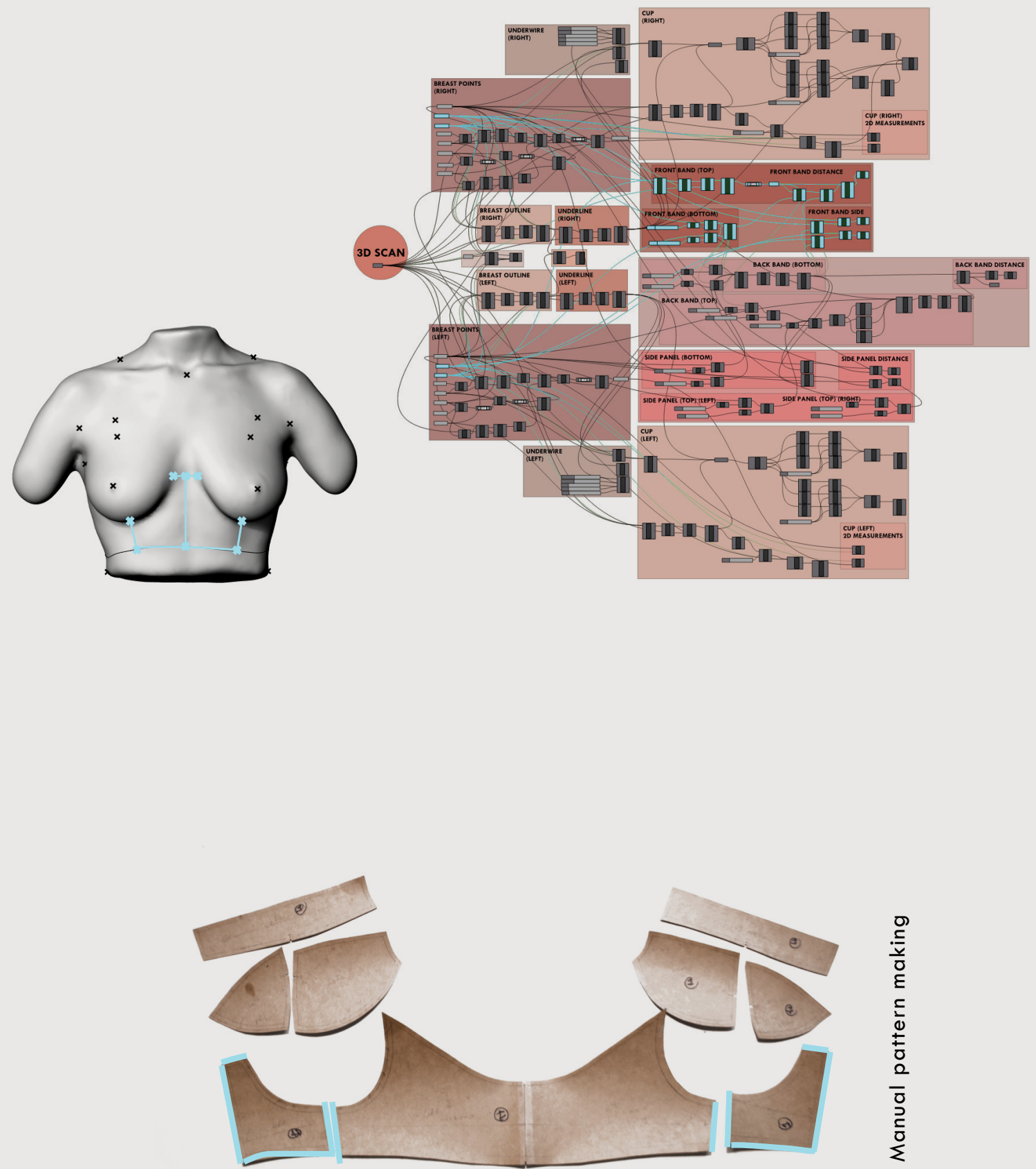

$\vec{\infty}$ 


\section{S I D E P A N E L}

LENGTH: OBP \& SPBP
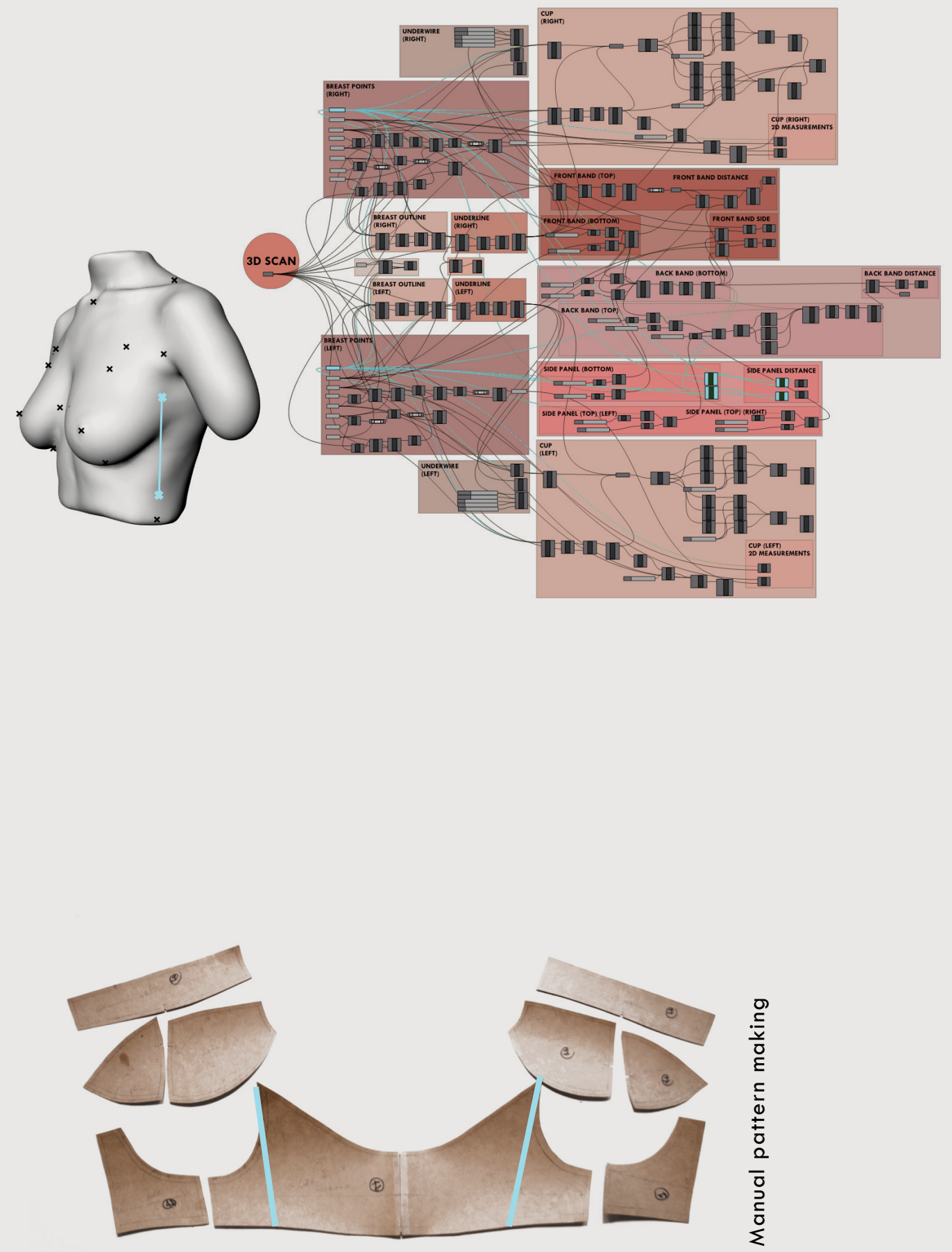


\section{B A C K B A N D}

LENGTH: BBTP TO BBBP
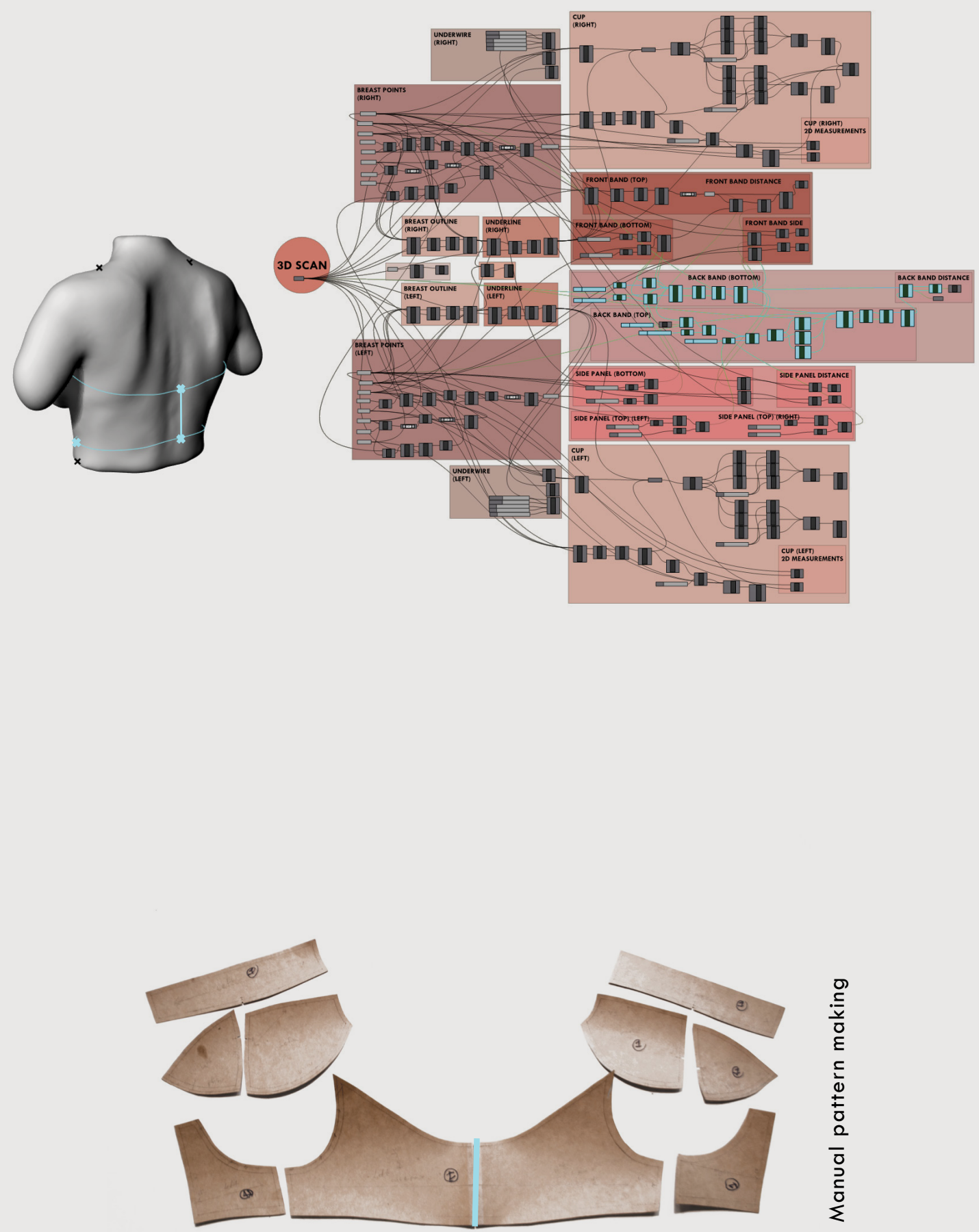


\section{O VER B U S T \& U N D E R B U S T}
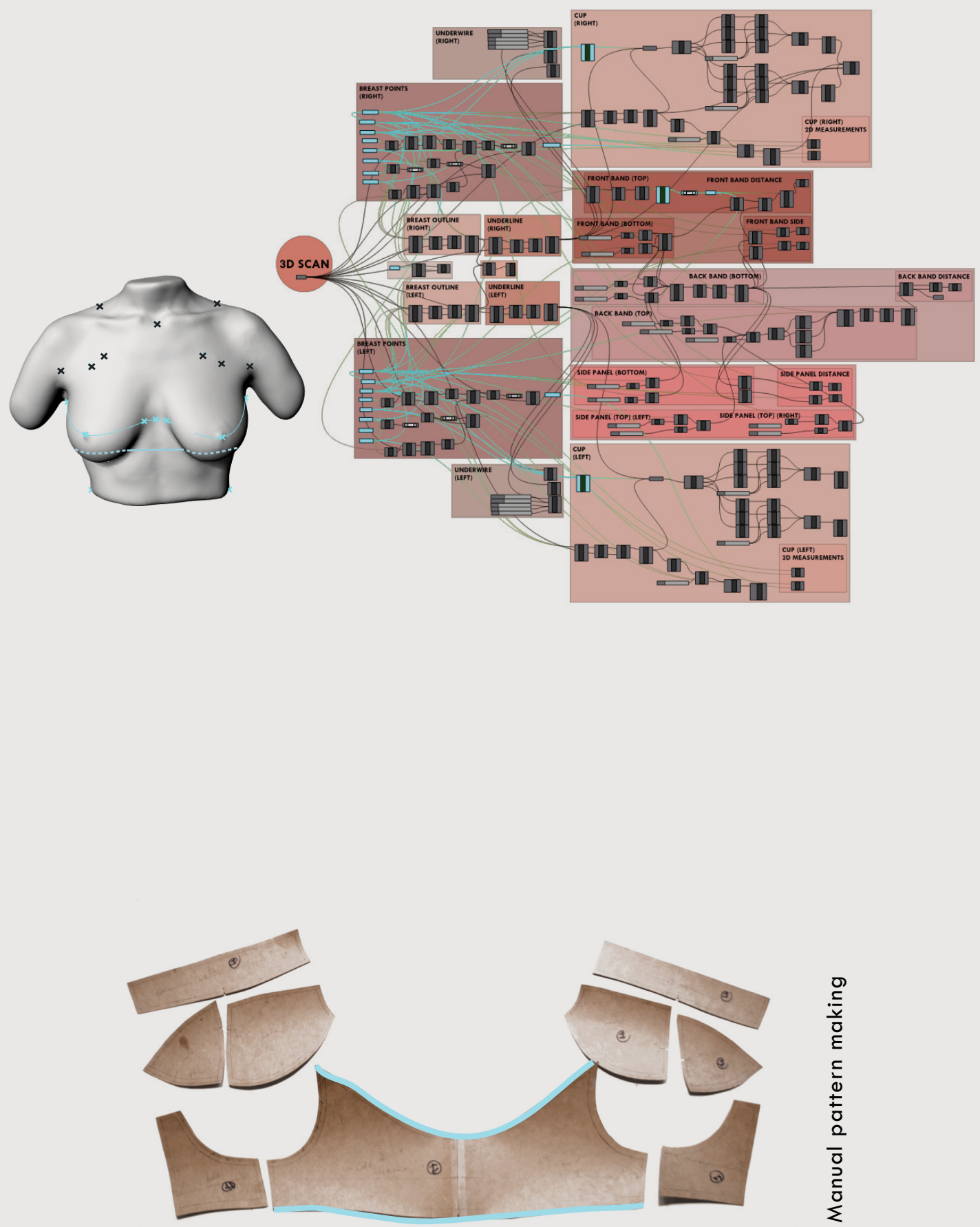


\section{Application}

The Scan to Sew method, as with Scan to Knit design process, depends on a second step measurement to account for material stretch. In this case, while drawing the patterns, an additional $2 \mathrm{~cm}$ was added to provide for stitching. This extra allowance then folded into the seams so the actual body of the bra would have the appropriate size. The underwire had a major role in the construction of the patterns. The underwire was needed to be developed first so that it could be used as a reference to create the patterns of the bra design. A 3D printed multi-material print of an underwire was fabricated based on the curve of the participant's actual underline breast curve (a measurement obtained from the parametric measuring system). The underwire was composed of two parts, an inner, firmer plastic base, and a softer flexible outer casing. These two parts created a structured yet highly flexible underwire alternative, which was designed to allow for better movement, while wearing the bra, as well as more comfortable fit compared to the rigidity of traditional metal underwire. Another notable design decision was the application of seam structures within the bra piece. Seams were added under the bust area for each breast, in the front of the band section. These two seams mirrored the same measurements gathered through landmarking points BBP to FBSP. The seams offered a more structured front band and better support for the base of the bra. Vertical seams were added to the cups, as discussed previously, to aid in cup shape, as well as breast shape. Lastly, materiality was closely considered for each part of the bra. There was a continuation of material exploration through merino knit. The merino knit was used for the cup design (as investigated in Chapter 08). However, since a fabric was selected for the design instead of yarn (for a 3D knitting process), a very fine gauge knit merino could be used to accentuate softness, breathability and stretch. A thick cotton fabric was chosen for the band to allow for the best support structure. For the side panels, mesh was applied to allow for a release of pressure on the side of the body and under the arms. The side panels were designed to draw back to the middle part of the back of the band, which was created to be a low cut and allow for room for shoulder blade movement. 

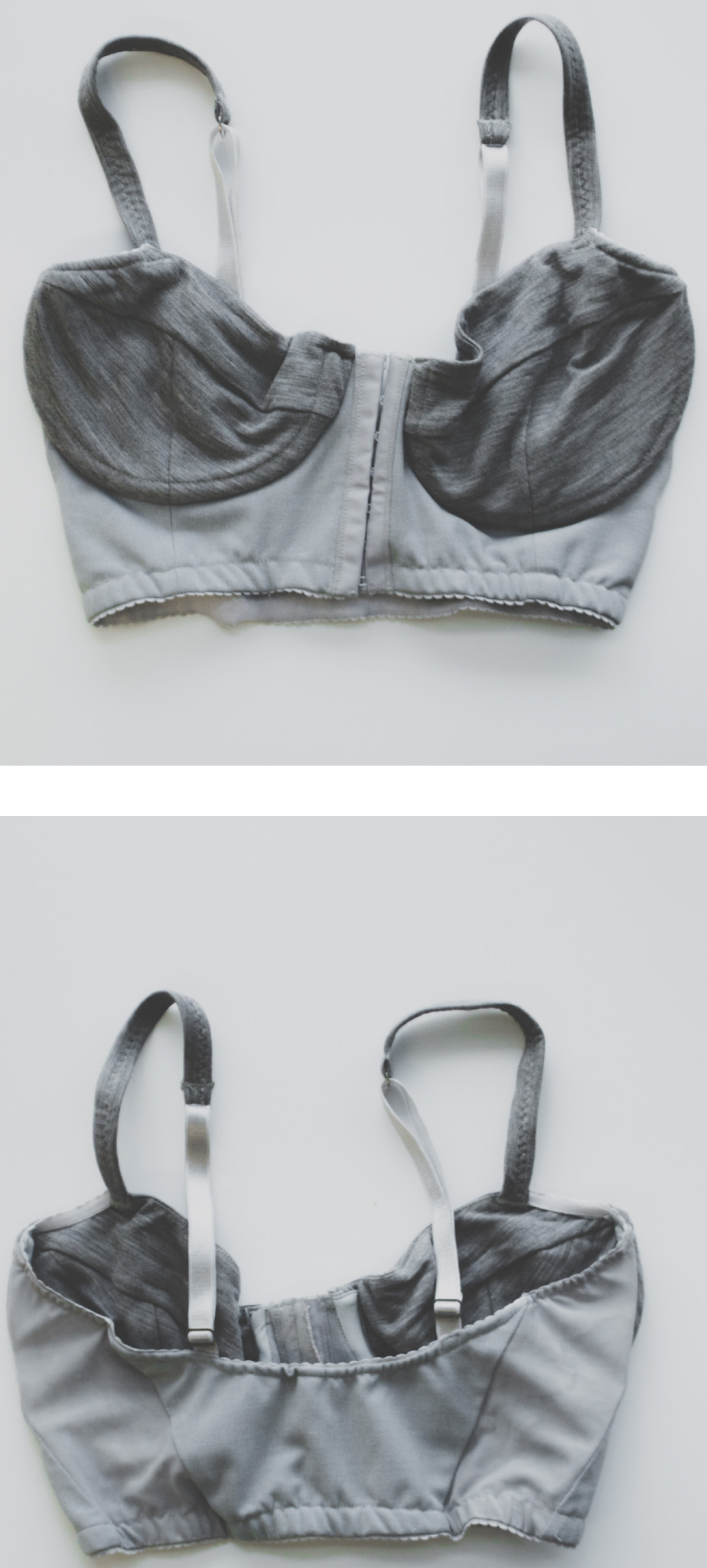


\section{COMPARING \\ MEASURE MENTS}

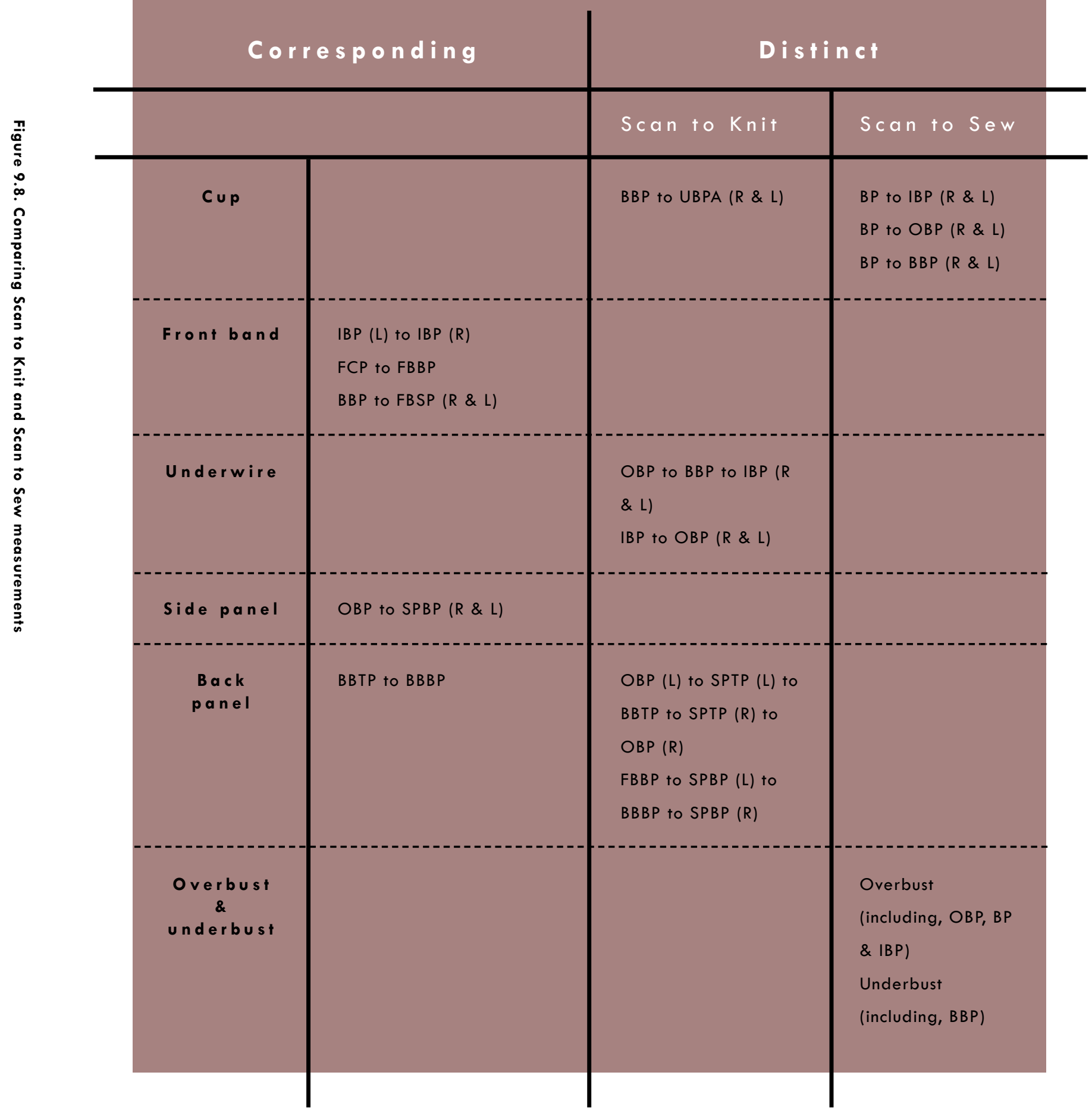




\section{$C O M P A R I N G$ \\ $M E T H O D S$}

\begin{tabular}{|c|c|c|}
\hline & Scan to Knit & Scan to Sew \\
\hline Benefits & $\begin{array}{l}\text { - Alternative design to } \\
\text { traditional underwire, through } \\
\text { the use of experimental } \\
\text { knitting structures (ridging } \\
\text { techniques) as well as } \\
\text { pemotex yarn which create } \\
\text { a solid but comfortable } \\
\text { structure. } \\
\text { (Almost) seamless design, } \\
\text { the lack of seams releases } \\
\text { unwanted pressure against } \\
\text { the body. } \\
\text { 3D shaping of the cup } \\
\text { structure without the use of } \\
\text { seams (2D structures sewn } \\
\text { together). } \\
\text { Specific knit structures to add } \\
\text { more or less stretch where } \\
\text { desired. } \\
\text { An overall light appeal and } \\
\text { feel. } \\
\text { Little to no material waste in } \\
\text { production. } \\
\text { Short production time. }\end{array}$ & $\begin{array}{l}\text { - Quite easy to adapt } \\
\text { measurements into the final } \\
\text { design. } \\
\text { - Ability to work with a variety of } \\
\text { different types of material. } \\
\text { Curves are much easier to } \\
\text { incorporate into measurements. }\end{array}$ \\
\hline Limitations & $\begin{array}{l}\text { - The ability to accurately } \\
\text { implement the underlying/ } \\
\text { underwire measurement. } \\
\text { Though an underwire has } \\
\text { been successfully knit using } \\
\text { specific experimental knitting } \\
\text { techniques, the desired shape, } \\
\text { length and accurate measure } \\
\text { is unsuccessful. This is the most } \\
\text { important limitation because } \\
\text { it can influence the fit of the } \\
\text { cup and band as well. }\end{array}$ & $\begin{array}{l}\text { - Skilled sewer with a high } \\
\text { expertise in cut and sew bra } \\
\text { design needed. } \\
\text { Time consuming process. } \\
\text { Though the measurements were } \\
\text { provided, a unique selection } \\
\text { of patterns needed to be } \\
\text { drawn and formulated for each } \\
\text { measurement change. } \\
\text { Dependency on physical } \\
\text { underwire to create bra pattern } \\
\text { against it. } \\
\text { Seams are necessary for design } \\
\text { production. }\end{array}$ \\
\hline
\end{tabular}


CHAPTER 09

BRA DESIGN DEVELOPMENT:

UTILIZATION OF MEASUREMENTS

\section{DISCUSSION}


This chapter sought out to validate the parametric measuring system developed by the author by demonstrating and comparing two different manufacturing methods of producing a designed bra utilizing measurements obtained from the system. The first method, Scan to Knit, involved the digital knit programming and 3D knitting machine (as explored in Chapter 08) to knit a single bra piece, the second, Scan to Sew, was the traditional cut and sew method of creating multiple bra patterns and sewing them together. Though both methods set out to produce a bra for the same participant, and obtained the measurements needed for construction through the information developed in the same parametric system, each method has it's own set of measurement requirements, advantages and limitations.

One of the most valuable benefits of $3 \mathrm{D}$ knitting is that a combination of different knit structures and yarn types can be seamlessly knit together in one garment piece, versus traditional cut and sew which depends on sewing together different pattern pieces, these pieces may be of different structure and material properties but they are not seamless. This seamlessness allows for less pressure against the body all around. A major limitation of the Scan to Knit method prototype was that it did not account for all measurements proposed. The two measurements, underline breast curve, and side panel length, could not be accurately designed in the digital knit software and future developments suggest the development for an asymmetrical curve for the underwire, while still allowing appropiate seamless $3 \mathrm{D}$ shaped curve to be knit within it.

One of the greatest successes of a traditional cut and sew process is that the design is not limited by what a machine can create (knitting is dependent on the knitting machine's capabilities). There is no worry if one section of the bra will be able to knit seamlessly to another, or if the type of knit structure will change the shape and sizing of the piece. Each piece of the bra is independently cut and designed to another therefore each piece can be removed, altered and replaced. However, the greatest limitation was depending on the physical underwire instead of the measurement of the underline breast curve. The underwire itself was based off real body data, the participant's underline breast curve, and utilized the appropriate landmarking points, but its flexibility made it difficult to draw patterns based off it. The underwire was experimentally $3 \mathrm{D}$ printed using multi-material filament which sought to explore alternative ways to the traditional metal underwire. The flexibility was designed so that there would be more comfort and allow to move the body with ease when wearing the bra. But its flexibility also made it very easy to change in size and shape. Since the cut and sew method depends on the underwire shape for measurement, the versatility of the $3 \mathrm{D}$ printed underwire proved to cause some mishap.
By comparing the methods of both bra manufacturing processes and design techniques, the research was able to compare and contrast the use of the measurements needed and used for each method. Though each method of developing a bra prototype was completed in a distinct manner they both successfully utilized the measurements gained from the parametric measuring system. Some measurements were used for both methods, such as IBP to IBP, FCP to FBBP, BBP to FBSP, OBP to SPBP, and $\mathrm{BBTP}$ to $\mathrm{BBBP}$ which are interestingly enough all related to the front band, side panel and back band. The cup, however, was measured in quite different manners. For knitting, 2D measurements were used to grasp an understanding of length and width, where the underline breast curve was used for reference, and specific knitting structures were used to created a 3D form and cup shape. Yet, for the cut and sew method, three curve measurements all which reference the bust point (BP) were used to create three different patterns, which together, when sewn, make up a $3 \mathrm{D}$ shape for the cup design. 

C H A P TER 10

RES U LT S 
This chapter brings together the developments made through the two distinct manufacturing processes to develop two unique bra prototypes, Scan to Knit and Scan to Sew. Each prototype was assessed according to the modified criteria of the previous chapter. Figures 10.1 and 10.2. illustrate the results of Scan to Knit and Scan to Sew processes. In the next few pages, assessment of each criteria is presented, followed by participant feedback. 


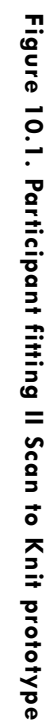
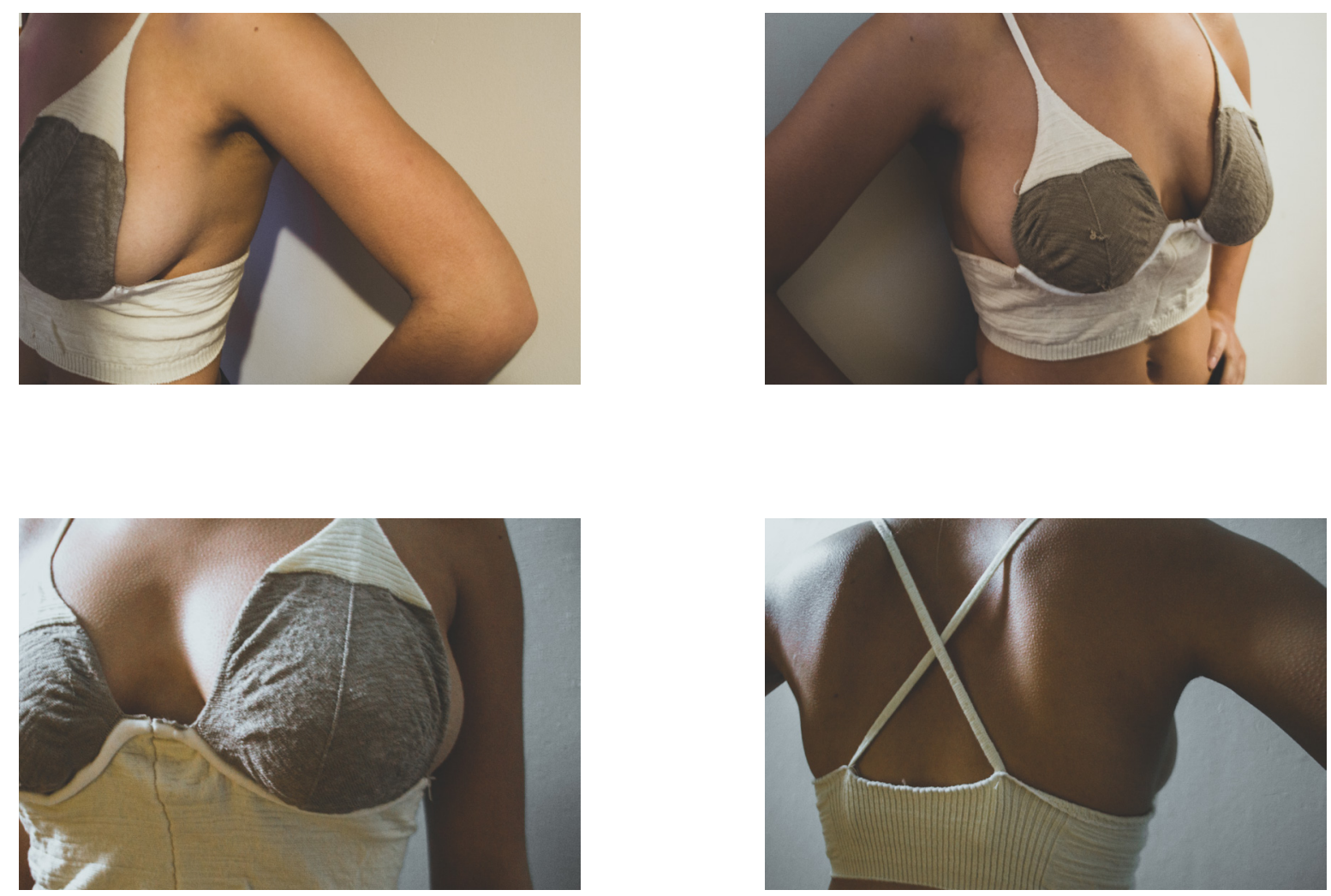

$\overrightarrow{0}$ 

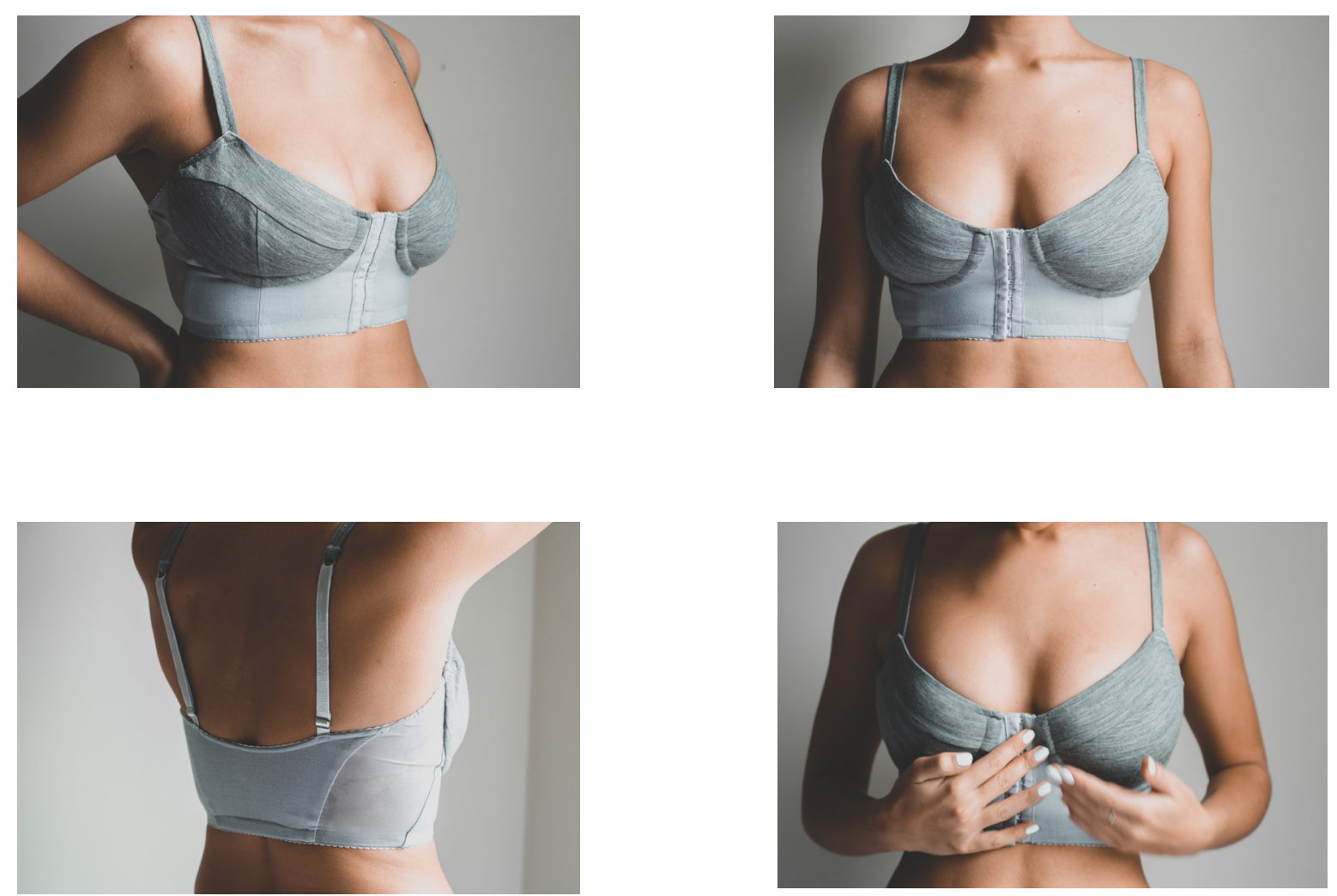

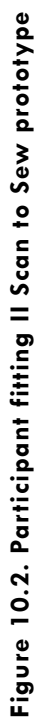




\section{R I TERIA A S SESSE D}

Criterio

Both prototypes, no matter manufacturing method, must acquire all measurements needed through the measuring system developed which uses Lee et al. (2004) landmarking points, as well as the additional points described by the author (Ch. 7 ).

The measurements used for the design must be taken for each breast individually; the design should not mirror left and right sides.

Design theme

Breast outline

Cup form
Breast outline

Cup form

Distribution of pressure
Once the measurements have been gathered from the measuring system, they need to be further adjusted depending on materials used to account for stretch.

For the Scan to Knit prototype the underwire must be represented seamlessly within the body of the bra. For the Scan to Sew prototype the underwire must be $3 \mathrm{D}$ printed using the exact underlying curve gathered from the measuring system.

$$
\text { - - - }
$$

Designed within the limitations of the 3D knitting software and machine capabilities for prototype $A$, or pattern-making and hand sewn process, for prototype $B$. The design of the bra must account for how each manufacturing processes are conducted, understand the limitations and benefits of each process.

Materials must be closely considered since the bra is a garment that is worn like a second skin, an itchy, unbreathable fabric will not only be uncomfortable but will irritate the breast area.

Design decisions must be made to enhance the support, structure and shape of the bra. For example, 3D knit structures can be designed to establish different types of material stretch and pressure. Additionally, a variety of seams and elastic lining can be developed in the Scan to Sew prototype for shape.

The bra design should be assessed by the fit criteria to make sure that the design itself is inherent to a good fit, and the wearer does not have any considerable adjustments to make

Longline design. The band must be extended long enough to aid in overall support.

Cup should lift and encase the breasts. The cups must be designed using knitting or sewing techniques to support the breasts appropriately.

Vertical seams should be utilized in the cup design. Vertical seams must be incorporated in most manufacturing processes to help shape the cups.
Breast outline

Cup form

Distribution of pressure

Breast outline

Cup form

$N / A$

Distribution of pressure

Cup form

Distribution of pressure 


\section{Scan to Knit}

Unsuccessful. One measure, the underline/ underwire curve measure (OBP to BBP to IBP [R \& L]) was not accurately utilized and had difficulty being programmed into the digital knit design.

Successful, as the right and left cup reflect differences in measurements between breasts. However, it is also unsuccessful in that the underwire did not reflect the correct measurements due to inability to draw underline/ underwire curve (as above).

Successful. The final measurements referenced a sample piece with correct knit structure and yarn type.

Successful. The underwire was knit seamlessly connected to the band, cup and front band.

Successful.

Successful. Merino and lycra combination was used throughout the bra prototype. Pemotex was seamlessly knit as an underwire alternative.

Successful. Specific knit structures were used throughout the bra, all unique to their purpose. Mis-knit for the side panel, $2 \times 2$ rib for back band, $2 \times 1$ rib for the hem, plain stitch for the bind off, lace stitch and ridged seam for the cups, and tubular for the straps.

$<<$ See table in the next page $>>$

Successful. Included a longline design.

Unsuccessful. Lack of appropriate underwire measurements have affected the cup shape so that the breasts are not encased appropriately.

Successful. A specific vertical ridged knit structure was used to shape and support the cups.

\section{$S e a n$ to $S \in W$}

Successful. All measurements used for the prototype were obtained from the parametric measuring system.

Successful. All measurements were gathered for each breast individually.

Successful. An added measurement was added to consider material stretch.

Successful. The underwire was 3D printed based on the actual breast underline measurements.

Successful.

Successful. Merino was used for the cups and straps, and a soft cotton was used for the band. Mesh was used for the side panel to offer additional flexibility and decrease pressure.

Successful. The pattern was cut with specific seams intended. Seams were used for the shaping of the cups, and, support for the front band and side panels.

$<<$ See table in the next page $>>$

Successful. Included a longline design.

Successful. Cup measurements encased the breasts appropriately.

Successful. Horizontal and vertical seams were used to shape and support the cups. 


\section{\begin{tabular}{llllll|llll}
$B$ & $R$ & $A$ & $F$
\end{tabular}}

\section{B and}

Primary support should come from the band

Does not ride up

03

Fitted on the loosest hook

04 Follows a horizontal line across the body

05 At most two fingers should be able to fit underneath

06 No flesh should bulge out

07 Fits firm

Allows for comfortable breathing

\section{Cups \& Underwires}

02 Breast does not bulge or spill over the cups

03 Cup has no wrinkles, gaps or spaces

04 Space on just the top of the cup suggests style flaw

05 Breast should be fully enclosed within the cup / smooth line

06 The underwire should not dig into the skin

07

Wire should not slip down

\section{Straps}

01 Do not dig into the skin / do not leave a red mark

02 Should sit firm, at most two fingers should be able to fit underneath

03 Should not slip off

04 Smooth line against the skin

\section{Front band}

\begin{tabular}{|c|c|}
\hline $\begin{array}{l}01 \\
02\end{array}$ & $\begin{array}{l}\text { Lie flat against the body } \\
\text { No gaps or space } \\
\qquad \text { Assymetry }\end{array}$ \\
\hline 01 & Fit to the larger breast \\
\hline
\end{tabular}




\section{B R A F I T C R I T E R I A \\ A S S E S S E D}

\section{Band | Cups $\mid$ Straps |Front band}

Les y m e try

Successful
for points

$01-04$,

$06,08$.

However,

05 and

07 call

for a firm

fit. The

knitted

band

should be

tigher. The

rib hem

is slightly

too

stretchy.

Successful

Successful

$\mid$

3
0
$n$
0
\pm
0
$u$
$n$
Unsuccessful for

points $2-3,5$, and 7 ,

since this is related

to the effectiveness

of the underwire

length which defines

the cup shape on

the sides. Since the

underwire curve

could not be knitted

to the accurate

measurements, the cup

could not fully encase

the breasts.

Successful for points 02 - 07.

Unsuccessful for point 01. See "front band" box to the right for comments.
Unsuccessful.

The flexibility

of the designed

underwires

led to a very

comfortable fit

all around the

cups but are

suggested to also

be the cause of

an unstable and

gaping front

band. The front

band did not

provide enough

structure to

secure the front

inner section

underwire in

place.
Successful for the

basic shape of

the cups. However, unsuccesful for

asymmetry in

general, due to

the inability to

utilize the accurate

measurements for

the underwire curve

in knitting. The

underwires were not

assymetrical and

therefore did not

connect to the cups

appropiately.

Successful. Each

cup was designed

based off

individual breast

measurements,

though there

was only slight

asymmetry, the left

cup and underwire

accounted for a

slightly larger

measurement. 
Both Scan to Knit and Scan to Sew bra prototypes were fitted and tested by the participant that the bra was designed for. The participant tried each one and discussed the successes and limitations of each prototype in reference to the different components of bra, including the different bra sections, shapes, structures, form and materiality with the author. 


\section{Scan to Knit}

\section{"S oft, flexible, calm and unique"}

The pemotex underwire was expressed as one of the main successes to this bra prototype. The pemotex allowed for a great amount of flexibility as well as comfort. It was hard for the participant to offer feedback regarding the cup region, as there lacked material to encompase the breasts sufficiently, though the participant did mention that the vertical seam on the cups was an improvement from the first prototype fitting (figure 8.7. and 8.8.). The merino lycra yarn type was favorable, the participant expressed that the material felt very light and noncompressing. The participant explained that the thickness of the merino material was great in that only one layer of material was needed to support and shape the bra. She compared it to her own bras which all have a lining layer as well as an outer layer. She preffered one layer as it felt closer to the skin as well as less restricting. Again, it was noted that if the cup/underwire region were knitted with the exact measurements, enough material to fully encase the breasts, the bra itself would have been much more successful. The band length was an improvement from the first fitting and offered better overall structure around the chest. Though she preffered the three strap design of the previous knitted bra prototype, she expressed this type of strap design seems to have a better lift, though she remarked it was difficult to efefctively critique without a full underwire and cup shape.

\author{
Scan to Sew
}

\section{"Warm, cosy, breathable, modern, \& refreshing"}

Straight away, the thin gauge merino fabric, used for the cups and the straps, stood out as one of the successes of this prototype. The participant remarked that the thickness was ideal and was not too padded for the cups, but shaped enough to sit nicely and form the breasts in a desired shape underclothes. The merino also helped the comfort of the straps against the skin and could be tight enough for support without cutting in. The underwire was also another point of satisfaction. She noted that the flexibility and softness of the wire provided ample comfort on the outer part of the cups, and allowed for easy movement under her arms. The participant expressed that the success of the underwire was that it was unnoticeable when worn, pressureless against the body, yet still helped in form a good enough shape for the cups, she expressed that she was "willing to sacrifice a total breast and cup lift for comfort [the underwire gave]". She claimed that the front band design was admirable, and explained that most front closure bras she encountered seemed unflattering, too traditional or even medical, such as post-mastectomy bras that close in the front. She explained that a new design of front closure bras was refreshing and a good idea as they are easier to put on and off. However, the front band on this prototype did gap where it should be flat against the skin, and explained that this section was the only real limitation in the bra design. The participant also suggested that the amount of hooks could be a point for future development, by reducing the number of hooks or a redesign of what type of hook or clasp can be used to connect the band. 


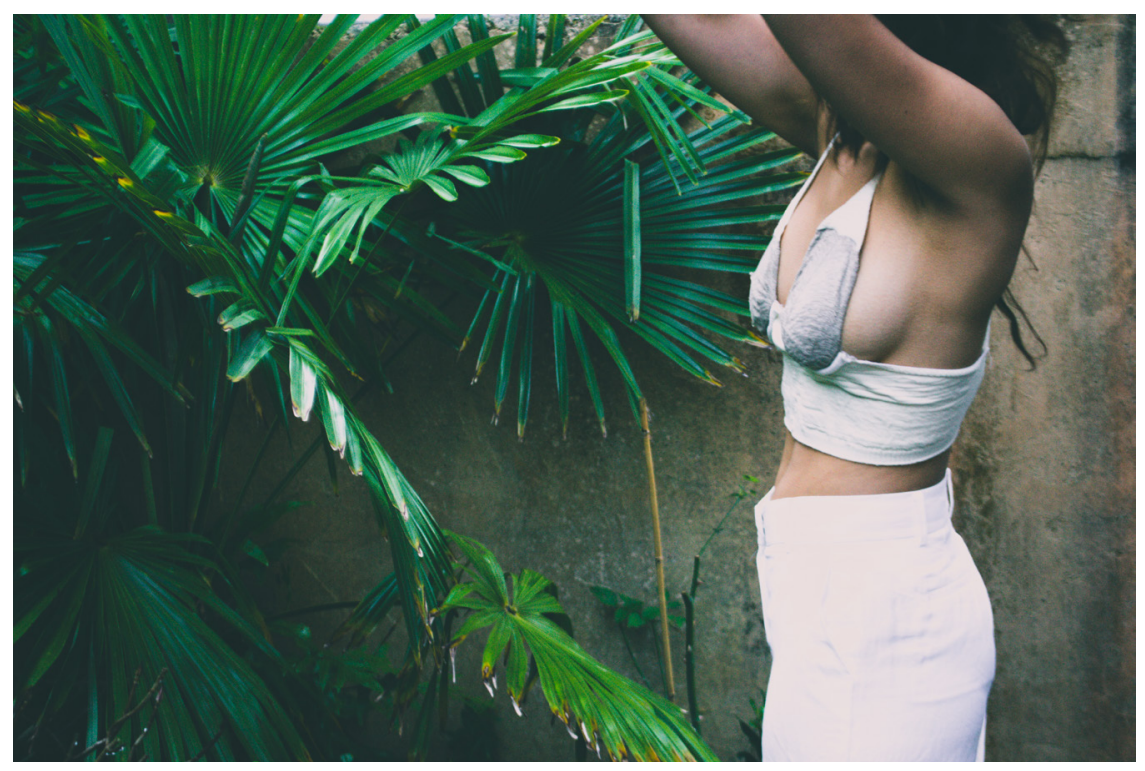

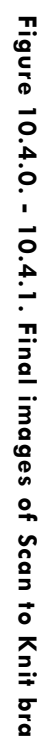

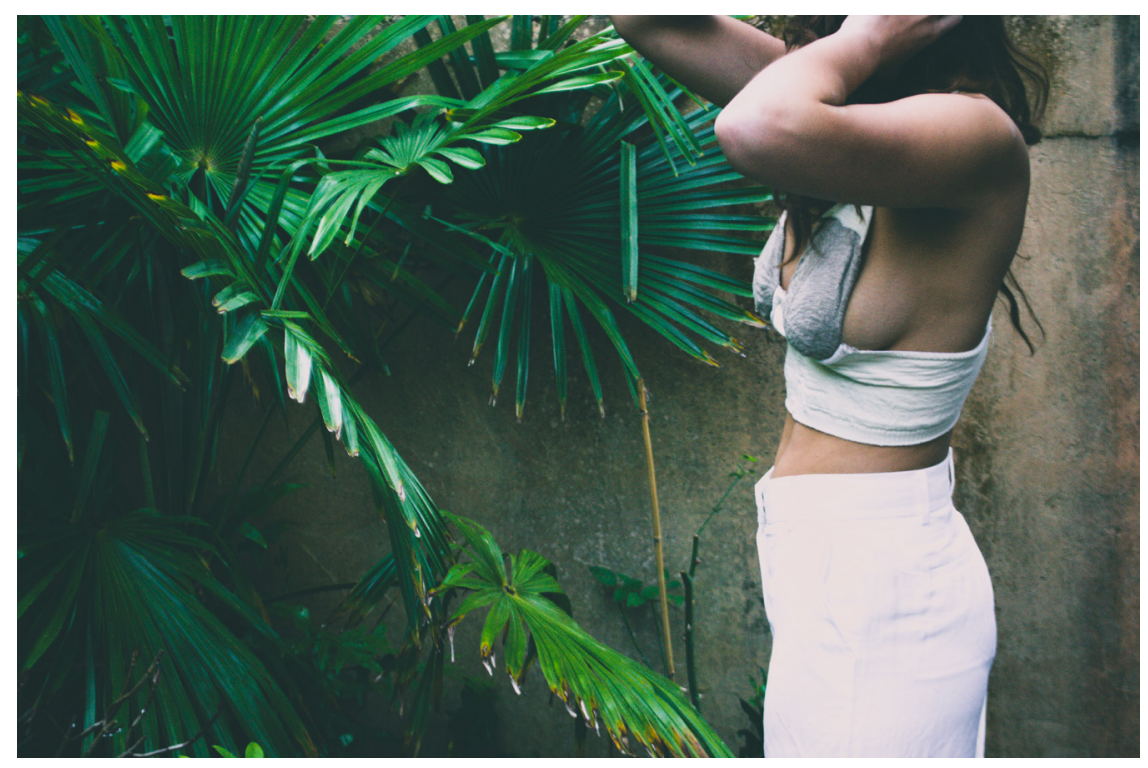

No 


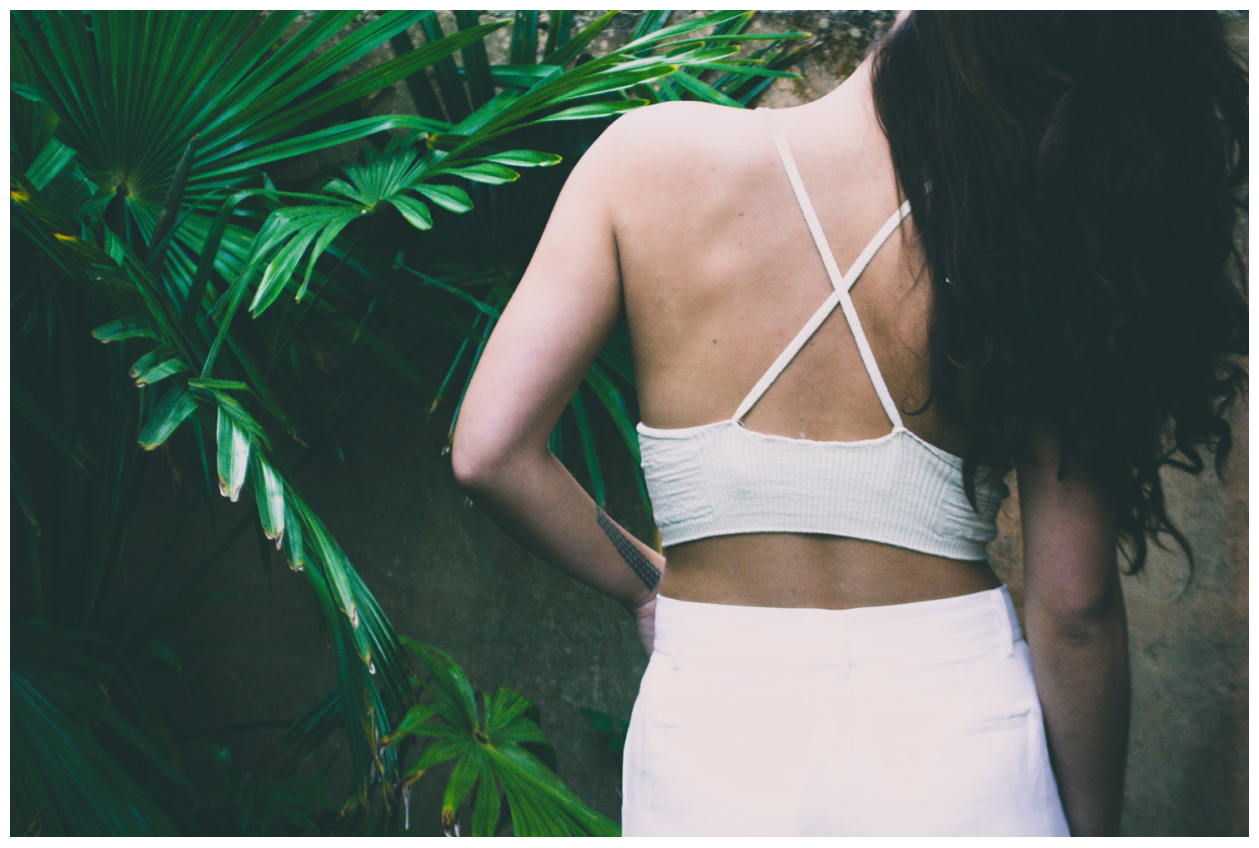

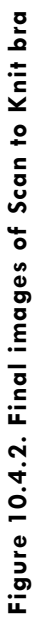




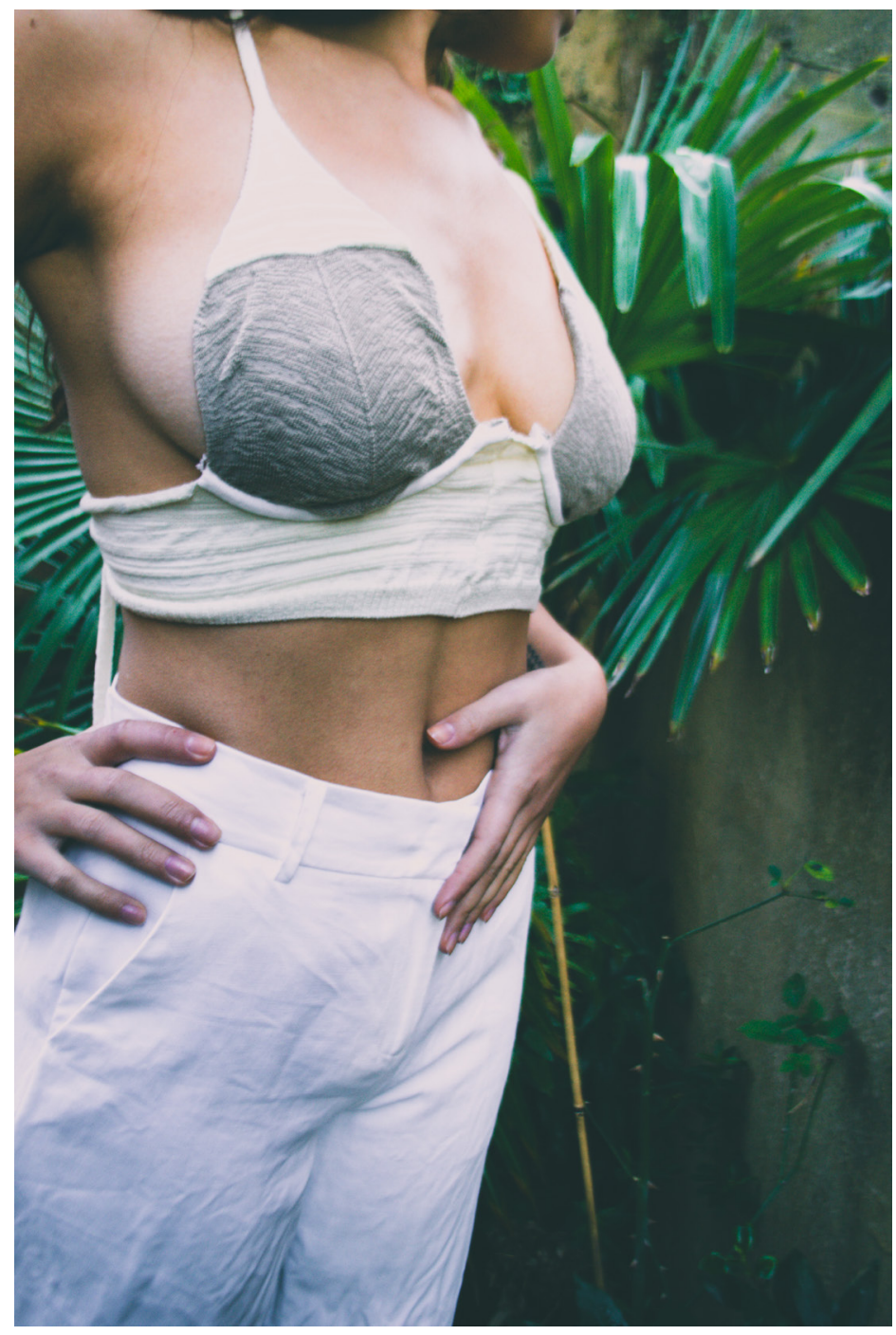

No 

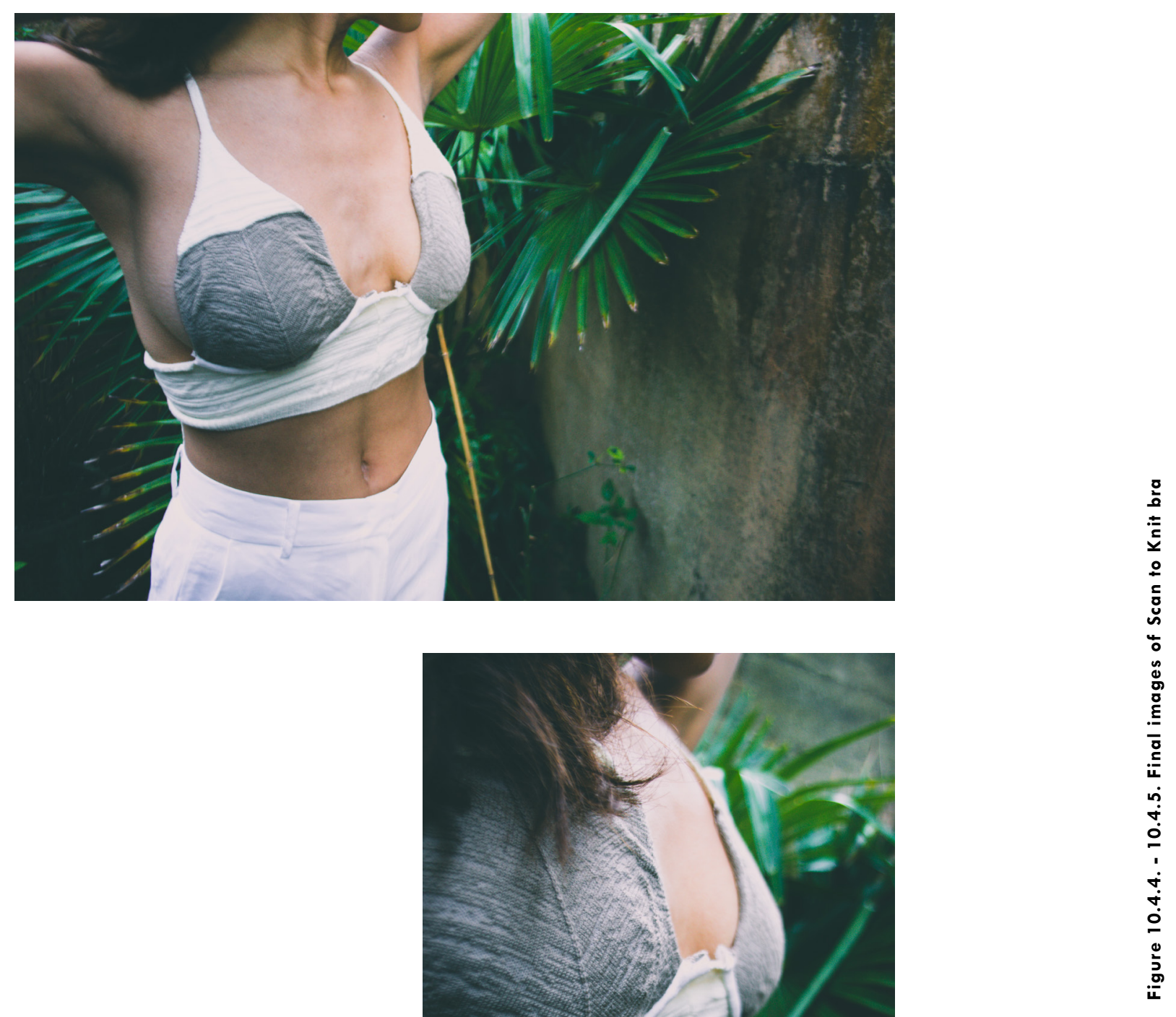

ำ 


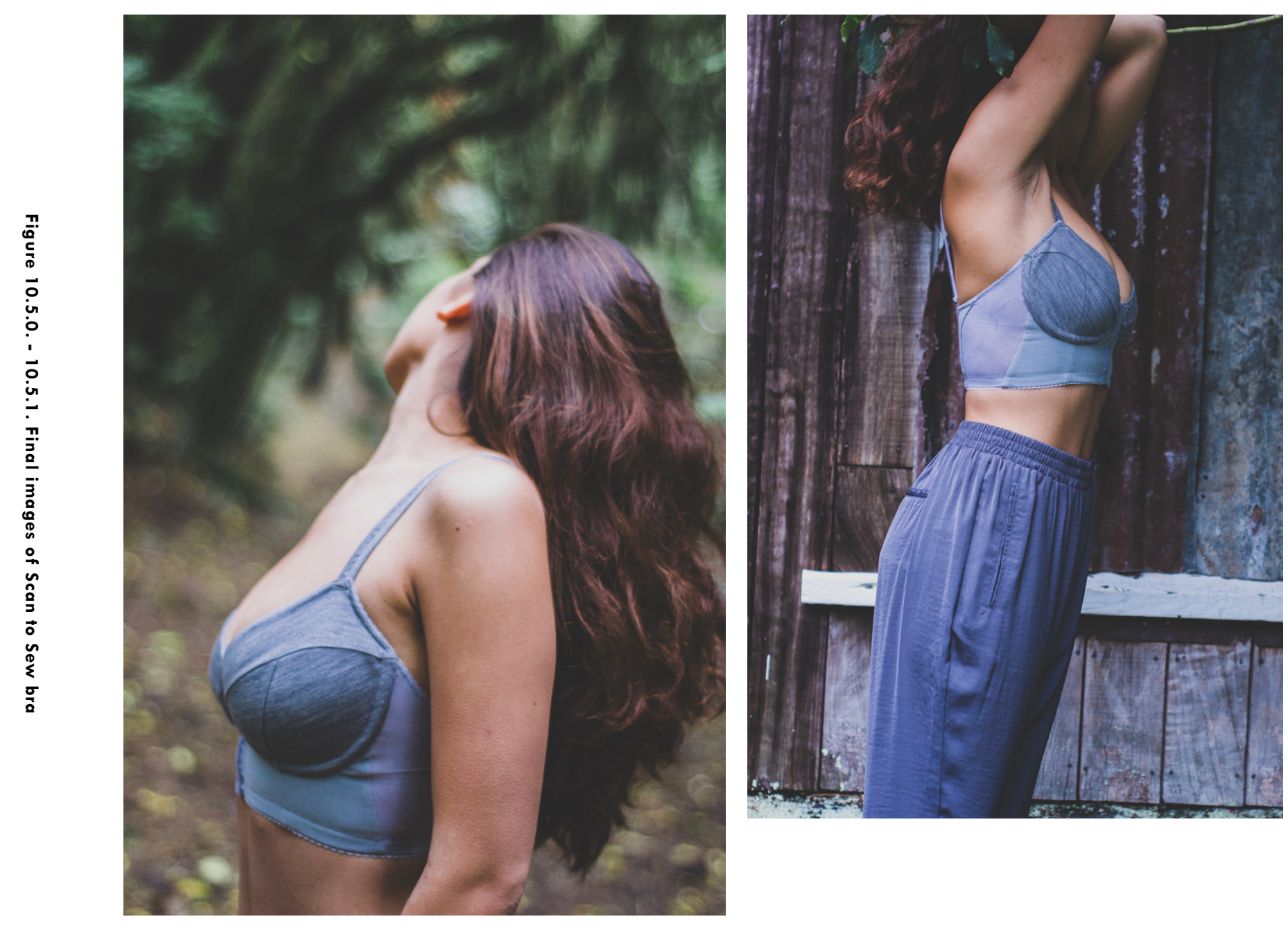

숭 

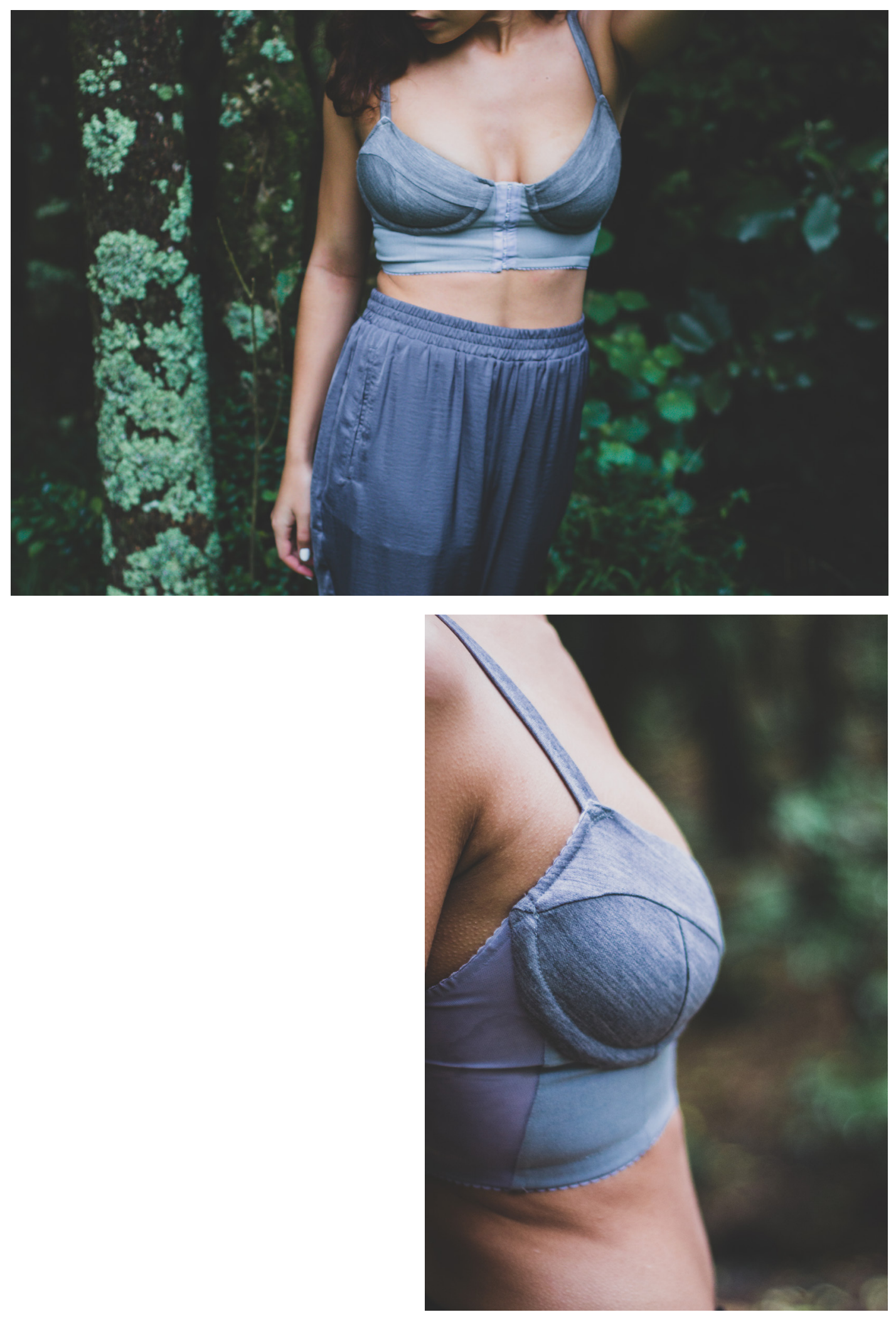

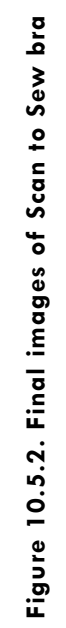




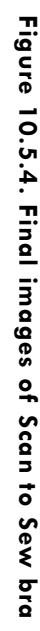

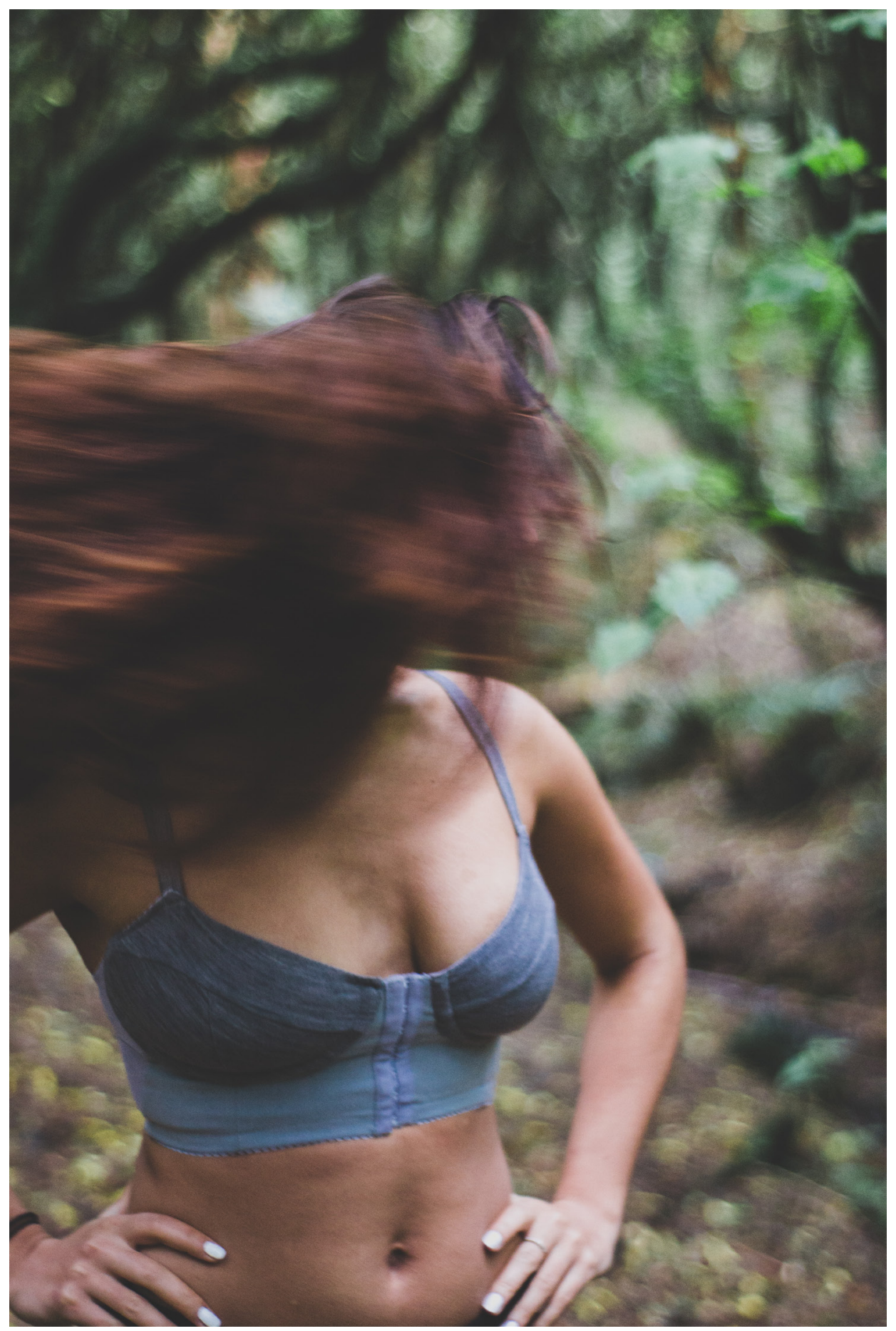

$N$
$\infty$ 

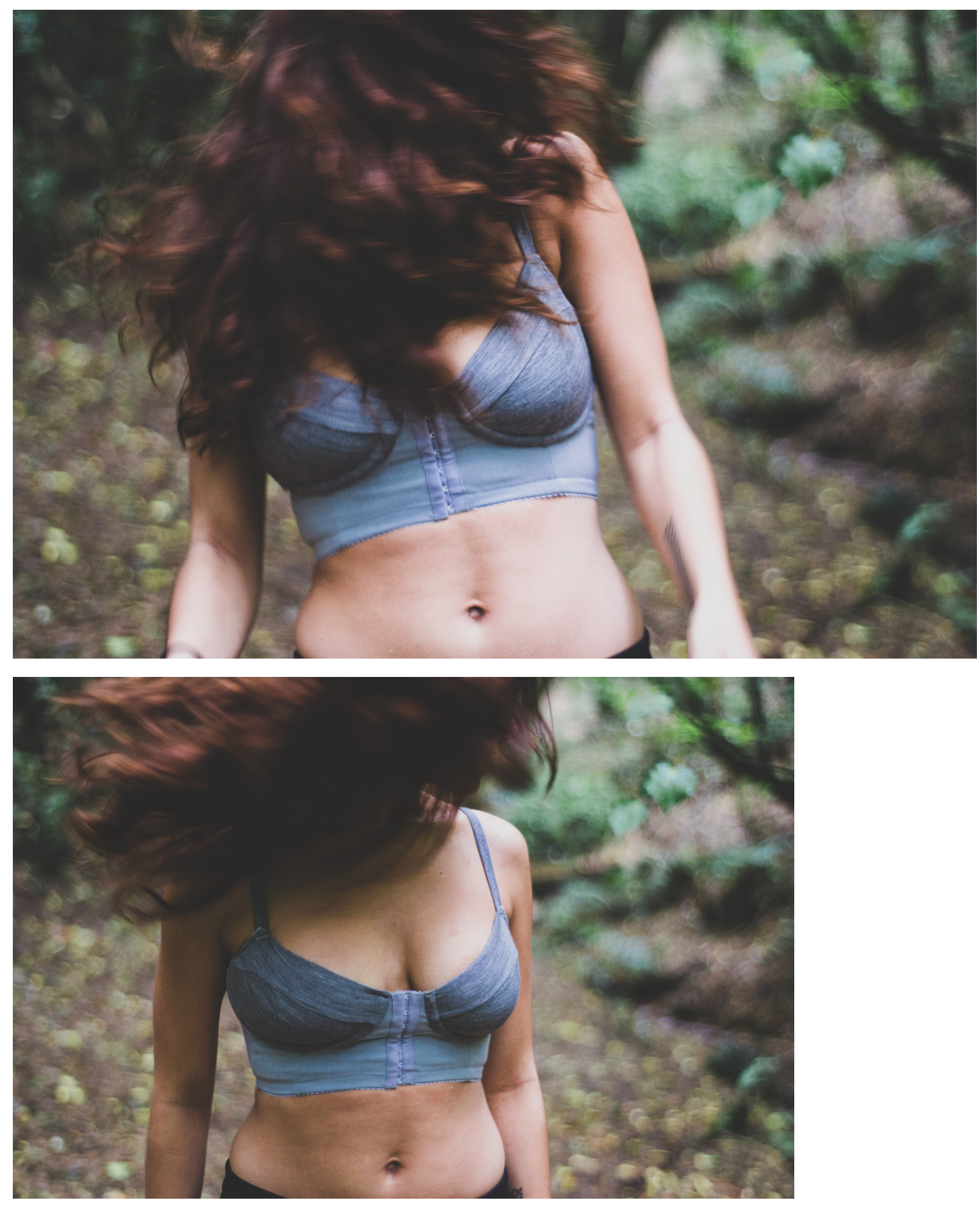

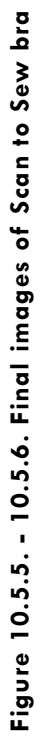

옹 

C H A P T E R 11

DISCUSSION\&

CONCLUSION 


\section{DISCUSSION}

This thesis explored a variety of avenues for design and concept of the bra, both as a product and as a future service. This exploration, in turn, helped develop a better understanding surrounding traditional, contemporary, and alternative approaches for improved fit and design. Volunteer participants played a vital role in the conceptual research, as well as helping steer research into a humancentered design approach. This will ensure that at each phase of investigation, the bra is conceptualised, designed and manufactured with the right human factor in mind. There are numerous applications to the process proposed in this research. Most bra design and manufacturing companies are seeking better ways of making bras that are aesthetically pleasing, are affordable and meet the changing demand (as well as body shape and contour) of their customers. The gap between what users of a bra need and require and what the bra manufacturers (and designers) provide is widening more than ever. This thesis by itself cannot revolutionized the process or even the culture of bra design. With manufacturing on one side and bra fit and selection on the other, it has opened new avenues of exploration for developing a process and concept for this important garment. The technical application, as will be discussed, requires further exploration and maturity.

Background literature has suggested a gap in breast measuring specifically in bra fit and design. The response to the lack of adequate measuring techniques is one of the key findings in this thesis, this has led to the development of an alternative method of measuring breasts, with benefits to bra design, and in the context of bra design an improved and individualised fit. The development of a digital parametric measuring system (Chapter 07) draws landmarking points and corresponding measurements based on an individualised 3D scanned digital mesh model of the person. Some landmarking points are fixed in placement to insure accurate referencing, and some points are flexible to allow for specific design interests. This system allows for necessary measurements to adapt to the individual $3 \mathrm{D}$ scan that is uploaded, all data is digitally stored so that the exact measurements can be referenced at a later point. Adaptive measurements are an essential part of the manufacturing system that are needed to mass produce a product (to keep the price relatively low), while attempting to respond to individual body types and consumers' sense of aesthetic and comfort. In the current market, mass produced bras (or essentially any product) are designed for a generic body types with discrete size steps. The advent of $3 \mathrm{D}$ scanning and $3 \mathrm{D}$ knitting and other advanced manufacturing technologies have allowed for a more readily available consumer product. As discussed in this research, many of these products are not sensitive to size fluctuation. However, in bra design and manufacturing, small fluctuation in size and fit can have a dire impact on comfort. Hence, the importance of this research is its contribution to adaptive measurement, gaps in measurements, and human factor.

3D scanning was a critical part to the entire research investigation. The scanning technique was utilized to gather a 3D model (mesh data) of the breast and upper torso, and in turn, draw measurements from. All succeeding landmarking points, measures needed for the design, and physical bra output stem from the necessity of an adequate $3 \mathrm{D}$ scan. The scans used for this research involved the author participating in capturing the scan for each participant. In this research, the breasts are scanned in a stagnant position. However as mentioned in the corresponding chapter, this is not the most optimal nor efficient procedure for scanning. The involvement of the author in the scanning process meant that there was a lack of privacy for the participant, as well as more trial and error to obtain a clean and useable scanned digital model. Further research is proposed to involve a body scanning system that is automated so that the participant, or consumer, will need only enter a private room, like a changing room, to be quickly scanned. Additionally, though the whole breast was accurately imaged into a usable 3D model, there is a chance to further research the desired shape of the breasts in the scanning process. In other words, to have the participant position their desired shape of their breasts, so that the corresponding measurements will adapt to it. A next step of investigation would be the incorporation of an adjustable, even disposable (one time use), bra that will be worn while being scanned for desired fit, until this process can be constructed in a digital manner, using algorithms to understand, or predict, how the breasts will behave within the desired bra shape. This would respond to the limitations in thesis research of not being able to account for variability in measurement due to breathing and posture. 
Literature suggests that ill-fitted underwires are the cause of discomfort and lack of adequate support in bras. Interviews and questionnaires with participants in this research also expressed that underwires were a source of pain and frustration in bras. This thesis responded to this issue through the development of of an underwire based on the breast outline which used the measurements gathered from the utilization of the $3 \mathrm{D}$ scan and digital measuring system, and produced through multi-material 3D printing. Multi-material 3D printing and design based on measurements from the system designed, led to a light and flexible but still structured support system. A direction for future research is a further investigation of the design and materiality of the underwire through $3 \mathrm{D}$ printing. This thesis undertook a limited exploration of underwire design through 3D printing by using multimaterial fabrication. However further research has the chance to redefine the underwire in terms of meaning, shape, and structure. This thesis experimented with a stiff base and a flexible outer layer, but the resulting underwire was limited by not holding adequate structure in certain areas. An investigation of understanding how multimaterial printing can influence individuality in underwire design, as well as utilize the various properties of design different sections depending on its need for strength, stiffness, flexibility and comfort.

The exploration of using 3D knitting in the context of bra design (Chapter 08) was a fascinating process filled with experimental techniques of utilizing the knitters capabilities to design and produce an alternative to traditional bra componentry. The iterations developed as a part of this research have led to pushing boundaries of what may be expected from a 3D knitted piece and tools to do it. One of the most intriguing aspects of the 3D knitting investigation was the construction of sections of seamless 3D forms within a flat piece, as seen through the development of the cup and underwire design. The 3D knitting technique of a 3D shaped cup offered an alternative to traditional cut and sew method of creating several patterns and sewing them together to create a $3 \mathrm{D}$ form. Participant interviews and questionnaires suggested that a soft or unpadded bra style was most popular, but current designs lack the desired breast shape when worn. The knitted 3D form of the cup was designed with a vertical knit structure to respond to the lack of shaping as well as limit the amount of seam structure as seen in most current un-padded bras. Additionally, the seamlessly knit underline with pemotex yarn and specific knit structure to create $3 \mathrm{D}$ ridging, was able to emulate the structure and support of a traditional metal underwire but also flexibility and softness against the body which offered a greater sense of comfort. This research explored the development of knitting a multi shaped garment, where a section may have a $3 \mathrm{D}$ form which seamlessly connects to a flat section, as in the bra prototype where the cup and underwire were 3D shaped and connected to a flat front band, side panel, back band and straps. This process also has the potential to be expanded to other areas in design. For example, currently, the popular Nike Flyknit shoes that are $3 \mathrm{D}$ knitted in a flat piece and sewn together, could potentially implement $3 \mathrm{D}$ shape structures for a more seamless fit.

As a result of the iterative process of developing a bra prototype, many avenues for further research have been noted that have not been able to be explored within this thesis. One of the most significant points is the relationship between scanning technology and digital knitting programming, in other words, creating seamless, three-dimensional shaping directly into the digital knit program. At the moment there is a disconnect between gathering appropriate body data and measurements (Chapter 07), and 3D knitting a physical bra outcome (Chapter 10). Though this thesis explored the utilization of measurements for 3D knitting, the transition from measuring software to knitting software was not seamless, and a manual consideration was needed (Chapter 09). The integration of $3 \mathrm{D}$ shaping in the digital knit programming will directly aid in the accuracy of measuring complexities, like the breasts, and limit manual intervention and reduce the risk for miscommunication from one software to the next. Further research is suggested to develop a plug-in between gathering 3D body data and inputting the curves and shapes directly into the knit program. Though at the moment, 3D shaping is possible to knit, they are currently designing in $2 \mathrm{D}$ and then controlled by specific knitting structures for $3 \mathrm{D}$ shape. A digital knit program that allows to design directly in $3 \mathrm{D}$ will allow for much more accurate bra design measurements and understanding of the resulting shape. 
$\stackrel{N}{D}$ 


\section{CONCLUSION}

Current bra fitting techniques do not appropriately consider individual and unique breast characteristics to improve bra fit, this critical oversight can lead to discomfort, pain and chronic health issues. Research has shown that for an accurate fit of a bra, detailed measurements of the breasts, and breast geometry, are necessary. This research focuses on developing an overall system for a more accurate bra design, instead of focusing on the product alone. The bra, consequently, becomes part of a life-long service system that is able to adapt to both physical and personal desire of the bra wearer. The research has led to the design of a bra fitting and design system that allows each user to be measured for her unique contour and individual body shape. Due to the symbiotic relationship between bra fit and bra design, the project's system has been designed parametrically in order to digitize the measuring process to insure that the points of measure adapt to each new scan and each woman is treated uniquely. Unlike traditional bra fitting methods, this system utilizes advanced technologies and digital manufacturing techniques, such as $3 \mathrm{D}$ scanning, to consider additional factors, not being currently considered, such as breast outline, shape, and asymmetry. Some degree of breast asymmetry is the norm of the female body, and this must be taken into account in accurate fittings. Through an iterative process, the research developed a series of prototypes that explored the distinctive qualities a $3 \mathrm{D}$ knitter can offer, such as specific knit structure and the development of seamless $3 \mathrm{D}$ form. The data accumulated would help produce tailored bra designs in order to insure a more precise bra fit. This thesis hopes to inspire future bra designs to utilize advanced digital technologies to facilitate improved bra fit through bra design, as well as, insure that the bra wearer is part of the conversation and has a say about this integral part of her everyday intimate attire. 

REFERENCES 


\section{LITER AT URE}

Apeagyei, P. R. (2010). Application of 3D body scanning technology to human measurement for clothing Fit. JDCTA International Journal of Digital Content Technology and Its Applications, 4(7), 58-68.

Black, S., \& Eckert, C. M. (2009). Developing Considerate Design: Meeting Individual Fashion and Clothing Needs Within a Framework of Sustainability. Handbook of Research in Mass Customization and Personalization (In 2 Volumes), 813-832.

Bowlexs, K., Steele, J. R., \& Munro, B. J. (2012). Features of sports bras that deter their use by Australian women. Journal of Science and Medicine in Sport, 15(3), 195-200.

Bremner, N. (2005). Seamless bodysize - technology round-up. Knitting International, 112, 26-29.

Bressler, K., Newman, K., \& Proctor, G. (1998). A Century of Style: Lingerie. Quarto Publishing Plc., London.

Bulstrode, N., Bellamy, E., \& Shrotria, S. (2001). Breast volume assessment: Comparing five different techniques. The Breast, 10(2), 117-123.

Bye, E., Labat, K. L., \& Delong, M. R. (2006). Analysis of Body Measurement Systems for Apparel. Clothing and Textiles Research Journal, 24(2), 66-79.

Chan, C. Y., Yu, W. W., \& Newton, E. (2001). Evaluation and Analysis of Bra Design. The Design Journal Design J, 4(3), 33-40.

Chen, C., Labat, K., \& Bye, E. (2011). Bust prominence related to bra fit problems. International Journal of Consumer Studies, 35(6), 695-701.

Chen, C., Labat, K., \& Bye, E. (2010). Physical characteristics related to bra fit. Ergonomics, 53(4), 514 524.

Chen, X. (2015). Advances in 3D Textiles. Woodhead Publishing Limited, Cambridge, UK.

Chen, X., \& Wang, J. (2015). Breast volume measurement by mesh projection method based on 3D point cloud data. International Journal of Clothing Science and Technology Int Jnl of Clothing Sci \& Tech, 27(2), 221-236.

Choice Magazine. (2005). Shadow shop: bra fitting services. Fit for what? Choice Magazine, $15-19$.

Cole, F. L. (1988). Content Analysis. Clinical Nurse Specialist, 2(1), 53-57.

Creswell, J. W., \& L., P. C. (2011). Designing and conducting mixed methods research. Los Angeles: SAGE Publications.

Devarajan, P., \& Istook, C. L. (2004). Validation of 'Female Figure Identification Technique (FFIT) for Apparel' Softwear. Retrieved 2011 5-October from NCSU Web site, http://www.tx.ncsu.edu/jtatm/ volume 4 issue 1 /articles/Istook/devarajan _full_106_04.pdf

Dewsnap, B., \& Hart, C. (2004). Category management: A new approach for fashion marketing? European Journal of Marketing, 38(7), 809-834.

Ebrahim, M. (2011). 3D Scanners: History, applications, and future. Review article, Assuit University.

Ebrahim, M. (2015). 3D Scanners techniques overviews. International Journal of Science and Research (IJSR), 4(10), 323-331.

Fong, L.W. (2014). Development of seamless knitted bra for optimum fit. (Doctoral Thesis, The Hong Kong Polytechnic University, Hong Kong, China). Retrieved from, http://hdl.handle.net/10397/6859

Freeman, M. (2008). Hermeneutics. In L. M. Given, The SAGE Encyclopedia of Qualitative Research Methods 385-388. Los Angeles, London, New Delhi, Singapore: Sage Publications.

Gabriel, A., Fritzsche, S., Creasman, C., Baqai, W., Mordaunt, D., \& Maxwell, G. (2011). Incidence of breast and chest wall asymmetries: 4D photography. Aesthetic Surgery Journal, 31, 506-510.

Gehlsen, G., \& Stoner, L. J. (1987). The Female Breast in Sports and Exercise. Sports Women Medicine and Sport Science, 13-22.

Greenbaum, A., Heslop, T., Morris, J., \& Dunn, K. (2003). An investigation of the suitability of bra fit in women referred for reduction mammaplasty. British Journal of Plastic Surgery, 56(3), 230-236. 
Hardaker, C., \& Fozzard, G. (1997). The bra design process: a study of professional practice. International Journal of Clothing Science and Technology Int Jnl of Clothing Sci \& Tech, 9(4), 311 -325.

Hart, C., \& Dewsnap, B. (2001). An exploratory study of the consumer decision process for intimate apparel. Journal of Fashion Marketing and Management: An International Journal Jnl of Fashion Mrkting and Mgt, 5(2), $108-119$.

Hague, R. (2005). Unlocking the Design Potential of Rapid Manufacturing, in Rapid Manufacturing: An Industrial Revolution for the Digital Age (eds N. Hopkinson, R.J.M. Hague and P.M. Dickens), John Wiley \& Sons, Ltd, Chichester, UK.

Hu, J.L. (2008). 3D Fibrous Assemblies. Woodhead Publishing Limited, Cambridge, UK.

Istook, C. L., \& Hwang, S. (2001). 3D body scanning systems with application to the apparel industry. Journal of Fashion Marketing and Management: An International Journal Jnl of Fashion Mrkting and Mgt, 5(2), 120-132.

Jenkins, S. P. (2005). Sports science handbook: The essential guide to kinesiology, sport and exercise science. Brentwood, Essex: Multi-Science.

Jin, Z., Yan, Y., Yu S., \& Tao, J. (2009). Study on prediction model for seamless underwear fitness in comfortable apparel pressure. 2009 International Conference on Machine Learning and Cybernetics 2, 828-832.

Karjaluoto, E. (2013). The design method: A philosophy and process for functional visual communication. New Riders.

Kayar, R., Civelek, S., Cobanoglu, M., Gungor, O., Catel, H., \& Emiroglu, M. (2011). Five Methods of Breast Volume Measurement: A Comparative Study of Measurements of Specimen Volume in 30 Masectomy Cases. Breast Cancer: Basic and Clinical Research , 5, 43-52.

Kim, D-E., LaBat, K., Bye, E., Sohn, M., \& Ryan, K. (2014). A study of scan garment accuracy and reliability. The Journal of The Textile Institute 1-9.

Kim, J. W., Lee, S. Y., \& Hong, K. H. (2000). Development of sensible brassiere for middle aged women. Journal of Korean Society of Clothing and Textiles, 24(5), 714-723.

Kovacs, L., Eder, M., Hollweck, R., Zimmermann, A., Settles, M., Schneider, A., \& Biemer, E. (2007). Comparison between breast volume measurement using $3 D$ surface imaging and classical techniques. The Breast, 16(2), 137-145.

Krippendorff, K. (1980). Validity in content analysis. In E. Mochmann (Ed.), Computerstrategien die kommunikationsanalyse, 69-112.

Lam, C. (2005). Seamless: technology, market, future. Melliand International: Worldwide Textile Journal $8(3), 199-200$.

Lee, H., \& Hong, K. (2007, 05). Optimal brassiere wire based on the 3D anthropometric measurements of under breast curve. Applied Ergonomics, 38(3), 377-384.

Lee, H., Hong, K., \& Kim, E. (2004). Measurement protocol of women's nude breasts using a 3D scanning technique. Applied Ergonomics 35, 353-359.

Liang, X.(2008). An investigation into the pressures and sensations caused by wearing a bra and the influence of these on bra fitting. (Doctoral Dissertation, De Montfort University). Retrieved from, http://hdl.handle.net/2086/4810

Losken, A., Seify, H., Denson, D. D., Paredes, A. A., \& Carlson, G. W. (2005,). Validating Three-Dimensional Imaging of the Breast. Annals of Plastic Surgery, 54(5), 471-476.

Manzini, E., \& Vezzoli, C. (2003). Product-service systems and sustainability: Opportunities for sustainable solutions. Paris: UNEP.

Martin, B., \& Hanington, B. M. (2012). Universal methods of design: 100 ways to research complex problems, develop innovative ideas, and design effective solutions. Beverly, MA: Rockport.

McGhee, D. E. (2009). Sports bra design and bra fit: Minimising exercise-induced breast discomfort. (Doctoral Thesis, University of Wollongong, Wollongong, Australia). Retrieved from, http://ro.vow. edu.au/theses/3854

McGhee, D. E., \& Steele, J. R. (2010). Optimising breast support in female patients through correct bra fit. Journal of Science and Medicine in Sport, 13, 568-572. 
McGhee, D. E., \& Steele, J. R. (2011). Breast volume and bra size. International Journal of Clothing Science and Technology Int Jnl of Clothing Sci \& Tech, 23(5), 351-360.

McCall, T. (2014). Can New Technology Help You Find a Better-Fitting Bra? Fashionista. Retrieved from, http://fashionista.com/2014/07/bra-fitting-technology

McKinnon, L., \& Istook, C. L. (2002). Body scanning. Journal of Fashion Marketing and Management: An International Journal Jnl of Fashion Mrkting and Mgt, 6(2), 103-121.

Mills, C., Risius, D., \& Scurr, J. (2014). Breast motion asymmetry during running. Journal of Sports Sciences, 33(7), 746-753.

Mitchell, N. A. (2013). Utilisation of 3D body scanning technology as a research tool when establishing adequate bra fit. (Master's Thesis, Manchester Metropolitan University, Manchester, United Kingdom). Retrieved from, http://hdl.handle.net/2173/314013

Mont, O. (2002). Clarifying the concept of product-service system. Journal of Cleaner Production, 10(3), 237-245.

Morris, D., Mee, J., \& Salt, H. (2002). The calibration of female breast size by modeling. Proceedings of International Foundation of Fashion Technology Institutes Conference, Hong Kong.

Oh, S. \& Chun, J. (2014). New Breast Measurement Technique and Bra Sizing System Based on 3D Body Scan Data. Journal of the Ergonomics Society of Korea 33, 299-311.

Page, K., \& Steele, J. R. (1999). Breast Motion and Sports Brassiere Design. Sports Medicine, $27(4), 205-$ 211 .

Pandarum, R., Yu, W., \& Hunter, L. (2011). 3-D breast anthropometry of plus-sized women in South Africa. Ergonomics, 54(9), 866-875.

Paquette, S. (1996). 3D Scanning in Apparel Design and Human Engineering. IEEE Computer Graphics and Application, 16(5) 11-15.

Peterson, J., Larsson, J., Mujanovic, M. \& Mattila, H. (2011). Mass Customisation of Flat Knitted Fashion Products: Simulation of the Co-Design Process. AUTEX Research Journal, 11(1), 6-13.

Raeve, A., Cools, J., Smedt, M.(2012, November). Mass Customization, Business Model for the Future of Fashion Industry. Paper presented at the 3rd Global Fashion International Conference, Madrid, Spain. Retrieved from, https://expertise.hogent.be/files/10254908/Mass_customization_business_ model_for_the_future_of_fashion_industry_full_text.pdf

Rong, Z. (2006). Breast sizing and development of 3D seamless bra. (Master's Thesis, The Hong Kong Polytechnic University, Hong Kong, China). Retrieved from, http://hdl.handle.net/10397/2619

Rose, T. (2016). The end of average: How we succeed in a world that values sameness.

Shin, K. (2007). Patternmaking for the underwired bra. New Directions, The Journal of The Textile Institute, $98(4), 301-318$.

Shin, K. (2009). The Origins And Evolution Of The Bra. (Doctoral Thesis, University of Northumbria, NewCastle, United Kingdom). Retrieved from, http://www.scribd.com/doc/284756290/The-originand-evolution-of-the-bra-Kristina-Woo-Kyung-Shin-2009-pdf

Simmons, K. P., \& Istook, C. L. (2003). Body measurement techniques. Journal of Fashion Marketing and Management: An International Journal Jnl of Fashion Mrkting and Mgt, 7(3), 306-332.

Smith, A. (2013). Seamless knitwear: Singularities in design. AUT University, Auckland, New Zealand. Available at, http://hdl.handle.net/10292/5761.

Spencer, D. (2001). Knitting Technology: A Comprehensive Handbook and Practical Guide. Woodhead Publishing Limited, Cambridge, UK.

Standring, S., \& Gray, H. (2008). Gray's anatomy: The anatomical basis of clinical practice. Edinburgh: Churchill Livingstone/Elsevier.

Tsarenko, Y., \& Strizhakova, Y. (2015). Insights into Gendered Consumption: Modeling Retailer Outcomes and Consumer Shopping Characteristics. Developments in Marketing Science: Proceedings of the Academy of Marketing Science Marketing in Transition: Scarcity, Globalism, \& Sustainability, 157-160.

Tseng, M., \& Jiao, M. J. (1996). Design for Mass Customization. Annals of the CIRP, 45(1), $153-156$.

Tuck, C.J., Hague, R., Ruo, M., Ransley, M. \& Russel, P. (2008). Rapid manufacturing facilitated customisation. International Journal of Computer Integrated Manufacturing, $21(3), 245-258$. 
Vignali, C., Vrontis, D., \& Vronti, P. (2004). Mass customisation and the clothing industry. Ekonomski pregled, $55(5-6), 502-512$.

Wang, L., Chen, D., \& Lin, B. (2009). Analysis of Pressure Distribution of Brassiere's Under Wires. Journal of Fiber Bioengineering and Informatics JFBI, 2(1), 19-24.

Wang, L., Chen, D., \& Lin, B. (2011). Effects of Side Strap and Elastic Hems of Bra Materials on Clothing Pressure Comfort. Journal of Fiber Bioengineering and Informatics JFBI, 4(2), 187-198.

White, J., \& Scurr, J. (2012). Evaluation of professional bra fitting criteria for bra selection and fitting in the UK. Ergonomics, 55(6), 704-711.

White, J., Scurr, J., \& Smith, N. (2009). The effect of breast support on kinetics during overground running performance. Ergonomics, 52, 492-498.

Wohleber, C. (2003). The Bra. American Heritage's Invention and Technology, 18(4), 1-2. Retrieved from, http: //www.inventionandtech.com/content/bra-0

Wood, K., Cameron, M., \& Fitzgerald, K. (2009). Breast size, bra fit and thoracic pain in young women: a correlational study. Chiropractic and Osteopathy, 16 (1), 1-7.

Wright, M.C.M. (2002). Graphical analysis of bra size calculation procedures. International Journal of clothing Science and Technology, $14(1), 41-45$.

Wu, L., Yick, K-L., Ng, S-P., Yip, J., \& Kong, K-H. (2012). Parametric design and process parameter optimisation for bra cup molding via response surface methodology. Expert Systems with Applications: An International Journal, 39(1), 162-171.

Yick, K. L., Ng, S. P., Zhou, X. J., Yu, W., \& Chan, D. (2008). Wire Frame Representation of 3D Moulded Bra Cup and Its Application To Example-based Design. Fibres and Polymers, 9(5), 653-658.

Yip, J., \& Yu, W. (2006). Intimate apparel with special functions. Innovation and Technology of Women's Intimate Apparel, 171-195.

Zheng, R. (2007). Breast sizing and development of 3D seamless bra. Hong Kong: Institute of Textiles and Clothing, The Hong Kong Polytechnic University.

Zheng, R., Yu, W., \& Fan, J. (2006). Development of a new chinese bra sizing system based on breast anthropometric measurements. International Journal of Industrial Ergonomics, 37(8), 697-705.

Zheng, R., Yu, W., \& Fan, J. (2009). Pressure evaluation of 3D seamless knitted bras and conventional wired bras. Fibers Polym Fibers and Polymers, 10(1), 124-131.

\section{FIGURES}

All figures, other than those listed below, were produced by the author.

2.1. True \& Co. Fit Quiz. (2016). True \& Co.. Retrieved from, https://trueandco.com/quiz

2.2. Your Perfect Fit. (2016). Victoria's Secret. Retrieved from, https://www.victoriassecret.com/bras/findyour-perfect-fit

2.3. Bra Size Calculator. (2016). Marks \& Spencer. Retrieved from, https://www.marksandspencer.com/s/ lingerie/be-inspired/bra-fitting-tool

2.4. Fit Finder. (2016). ThirdLove. Retrieved from, https://www.thirdlove.com/collections/bras\#fit-finder

2.8. Breast Shape Directory. (2016). ThirdLove. Retrieved from, https://www.thirdlove.com/pages/breastshape-dictionary 

A P P E NDIX 


\section{APENDIX I}

\section{NOTES ON BRA FIT CRITERIA \\ BASED ON INDUSTRY \& ACADEMIA}

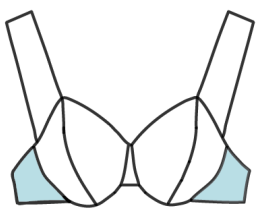

\section{ThirdLove}

+ You'll know the right band size when you can slip two fingers underneath the back of the band.

+ The band should be snug on the loosest hook, so when your bra stretches out you can continue to tighten it.

+ If your band is creeping up on the back then it's time to buy a smaller band size so that you have a snug fit.

\section{Triumph}

+ It is important that it fits firmly, parallel to the cups.

+ If the band rides up at the back it is probably too big. Please choose a smaller under bust size and go up a cup size.

\section{Victoria Secret}

+ If the band is too tight to easily slide a finger underneath it, your band may be too small.

+ Your band should lay even all around and be snug enough and parrallel to the floor.

+ If your band rides up in the back or is higher in the back, you may need a smaller band or replacement bra. 


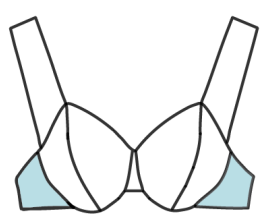

Bendon Lingerie, includes: Heidi Klum Intimates, Stella McCartney, Pleasure State, Lovable, Evollove, Fayreform, and Hickory.

+ Your underbust is the primary support for your bust, and is the anchor for your bra.

+ When your bra does not provide enough firmness and support for your underbust the back becomes looser, rides up, and your breasts sag.

+ Be sure to fasten it on the loosest, or middle, hook. If you can comfortably fasten it on the tigtest hook, try going down a back size.

\section{Bras $\mathrm{n}$ Things}

+ Check the band follows a horizontal line across your body and is not riding up at the back. If it is, go down a back size.

+ You should be able to get two fingers easily underneath.

+ Band rides up or sits too high: First check straps are not too tight. Decrease band size until it sits horizontally around the body, the back should be the same level as the front in the mirror.

\section{bras.co.nz}

+ The band is riding up your back. Your cups are too small, and you will need a larger cup size.

\section{Marks and Spencer}

+ The underband should be horizontal across your back.

+ It should be snug but with enough room to fit two fingers underneath it.

+ Go up a back size if the breast tissue under your arms is bulging over the underband.

+ Go down a back size if your breasts are showing beneath the underband and/or the underband is riding up your back.
+ The underband is designed and engineered to carry $80-85 \%$ of the weight of the breasts (Jonsson, 2013)

+ The underband should be snug enough not to shift around, but still allow two fingers to be inserted under the hooks (Choice Magazine 2005)

The underband is too small if flesh bulges over the back under band (Choice Magazine, 2005)

+ The back of the underband should sit securely and not ride up when moving the arms above the head (McGhee et al., 2008; Yu, 2011)

+ The back of the underband should be straight across and not ride-up or squeeze the flesh (Luciana \&t Voltz, 2009)

$+\quad$ The underband should be a firm fit but not uncomfortable (McGhee et al., 2008)

+ Tension in the underband should allow for comfortable breathing (Yu, 2009) 


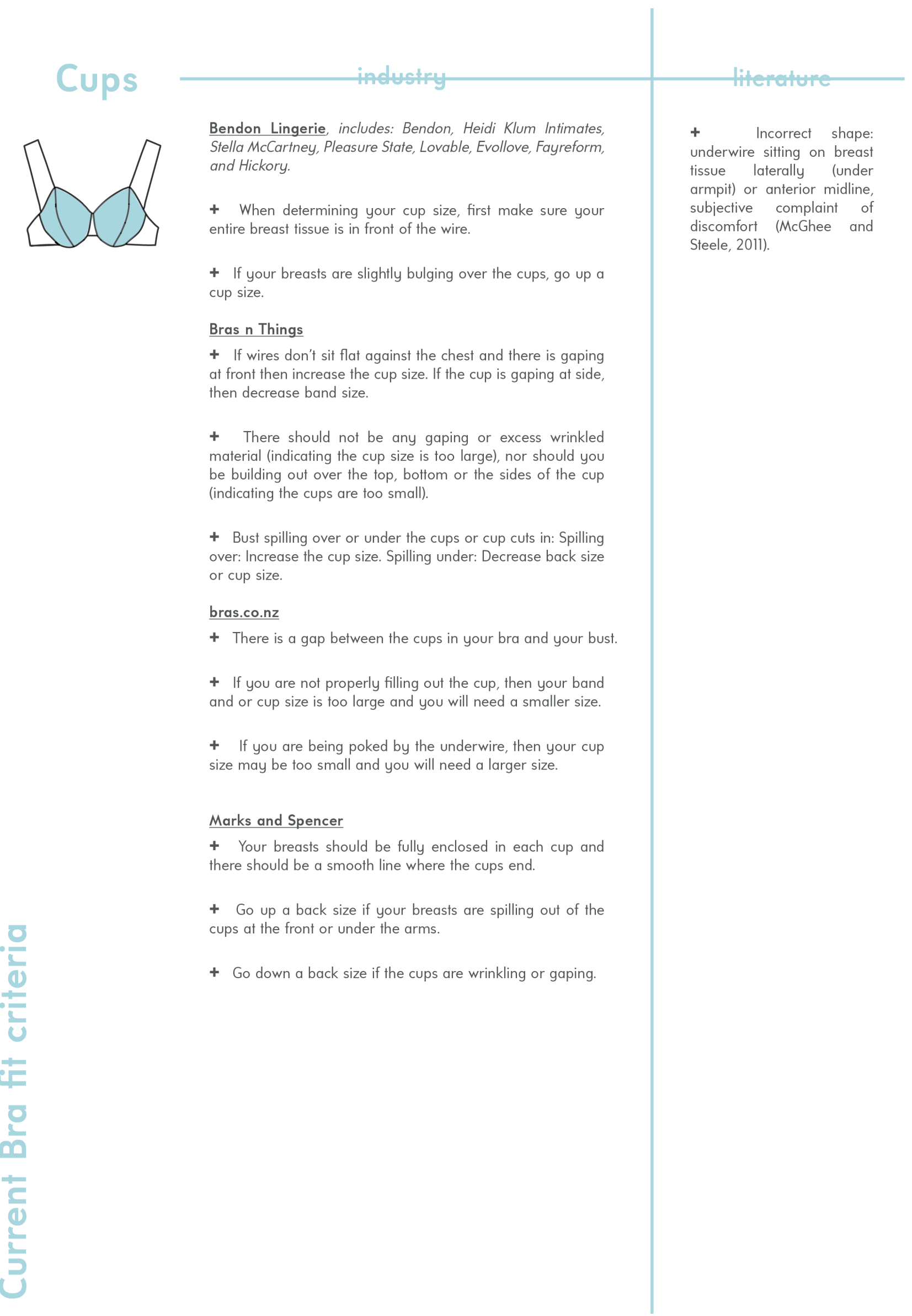




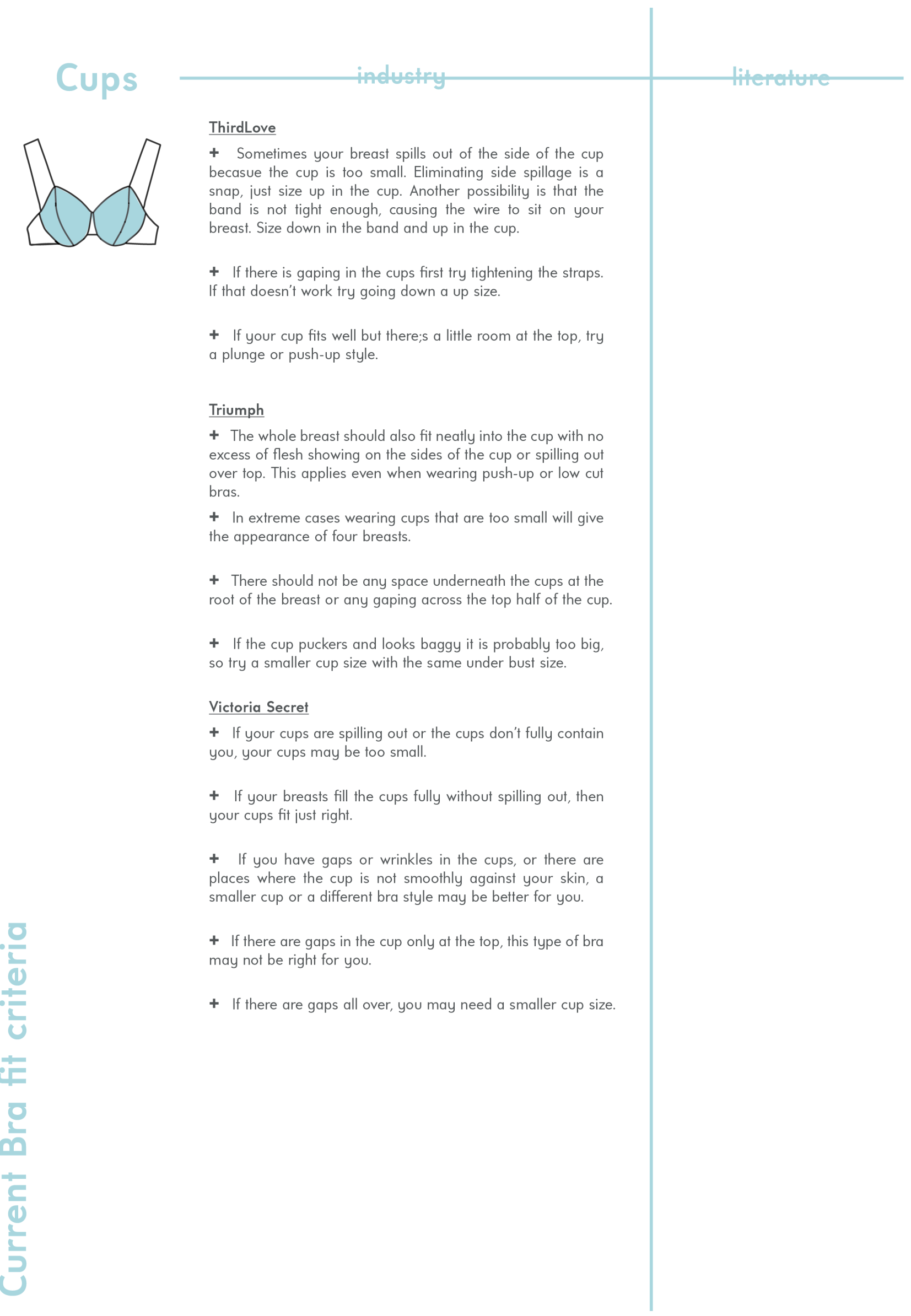




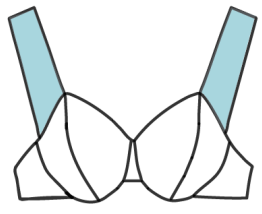

Bendon Lingerie, includes: Bendon, Heidi Klum Intimates, Stella McCartney, Pleasure State, Lovable, Evollove, Fayreform, and Hickory.

+ Each strap should rest on top of your shoulder without digging in- the weight of your breast should be on your bra's underbust, not your straps.

+ If you notice the strain is on your shoulders your bra's underbust is not doing its job.

+ You should only be able to fit no more than 3 fingers between the shoulder straps and your body.

If you can pull the shoulder straps further away from your body, make your straps firmer.

\section{Bras $\mathrm{n}$ Things}

+ Straps shouldn't leave a red mark.

+ If straps slip off your shoulders, either tighten straps or go down a band size.

+ You should be able to get two fingers easily underneath.

\section{bras.co.nz}

+ The straps are digging into your shoulders. You may need to loosen the straps. Or, your cups may be too small, pulling the bra downward and creating tension in the straps, you may need a larger cup size.

+ The straps are falling off your shoulders. Most likely, your band size is too large and you will need a smaller band. If you are not properly filling out the cup, then your band and or cup size is too large and you will need a smaller size. It is also possible that your bra has lost its elasticity.

\section{Marks and Spencer}

+ The bra straps should be fully enclosed in each cup and there should be a smooth line where the cup ends.

+ Loosen your straps if they are digging into your shoulders, and/or they feel tight and are casuing the cups to dig into your breasts.

+ Tighten your bra straps if they are slipping off your shoulders and/or you have narrow shoulders, avoid balcony bras as these have wide-set straps.
+ Shoulder straps should be snug without falling down or cutting into the skin (Choice Magazine, 2005)

$+\quad$ They should be firm but not digging in (McGhee \& Steele, 2011)

+ They should be wide enough and adjusted to comfortably provide breast support without digging into the shoulders - the straps should have room for adjustment in case they need to be tightened later (McGhee \&t Steele, 2011)

+ They should have the correct tension for breast support/ allow enough adjustment, but not too much turning/ strap should not fall off easily/ no cutting in the shoulder/ no fatigue (Yu, 2011)

+ Straps should lie perfectly in place without digging in (Luciani \& Voltz, 2009)

$+\quad$ The underband of a bra provides the majority $(80 \%)$ of support for the breasts, with the straps providing just (20\%) (Jonsson, 2013) 

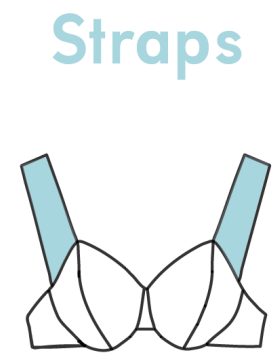

\section{ThirdLove}

+ If your straps are digging in chances are your band is too big or has stretched of to the point that you're not getting the support you deserve. Try buying one band size smaller.

+ If your straps are slipping, tighten the straps. We recommend tightening your straps every other month.

\section{Triumph}

+ They should sit parallel or slightly V-shaped on the back.

+ If the straps are digging into your shoulder it might be a sign that the under bust band is too big and not giving enough support. Try a smaller under bust size and a cup size bigger.

+ If the straps, after being adjusted, are slipping down your shoulders it might mean that the under bust band is too small. Try a bigger under bust size, and consider trying a cup size smaller.

\section{Victoria Secret}

+ If your straps dig in the band may be too large.

+ If your straps slip off a bra style with more strap options may help. 


\section{Hooks}
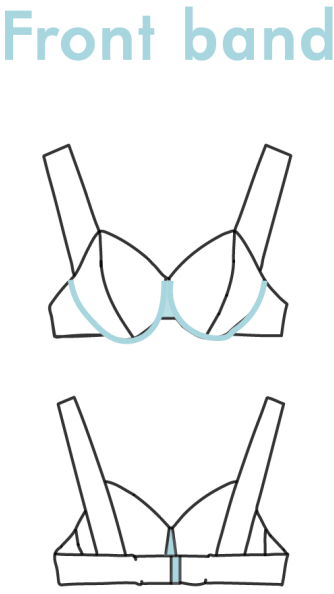

\section{UNDERWIRE}

Bras $\mathrm{n}$ Things

+ Wires should be flat against the rib cage and chest

\section{Marks and Spencer}

+ The wires at the front should sit flat at the breastbone between your breasts.

+ Go up a back size if the wires are digging into your breast tissue and/or the wires are liffing away from your breast bone.

+ Go down a back size if the wires are slipping down your body, towards your waist.

\section{Triumph}

+ The wire should go right around the contour of the breast sitting flat against your ribs without digging into the breast tissue.

+ If you feel the underwire is digging in between your breasts or in the breast tissue, try going one cup size bigger, but keep the under bust size the same.

\section{FRONT BAND}

Bendon Lingerie, includes: Bendon, Heidi Klum Intimates, Stella McCartney, Pleasure State, Lovable, Evollove, Fayreform, and Hickory.

+ The centre panel should lie flat against your ribcage.

\section{Triumph}

+ The centre front of the bra sits between the breasts and is also called a bra bridge. The bra bridge should lie flat against the body.

+ If there is a gap between the bra bridge and your rib cage, or the bra bridge sits on top of your breasts, it might mean that the cup size is too small. Try to go up a cup size, keeping the under bust size the same.

\section{FRONT BAND}

+ Too loose when not all in contact with the sternum (McGhee and Steele, 2011).

+ All of the front band should be in contact with the skin on the chest, not gaping away from the body (McGhee et al., 2008) 
Additional

bra fit criteria

suggestions

Assymetry

\section{Bendon}

+ Every woman has one breast that is slightly larger than the other. You should always fit for the larger breast once you determine your cup size below. 


\section{APENDIX II}

\section{D KNITTED ITERATIONS}

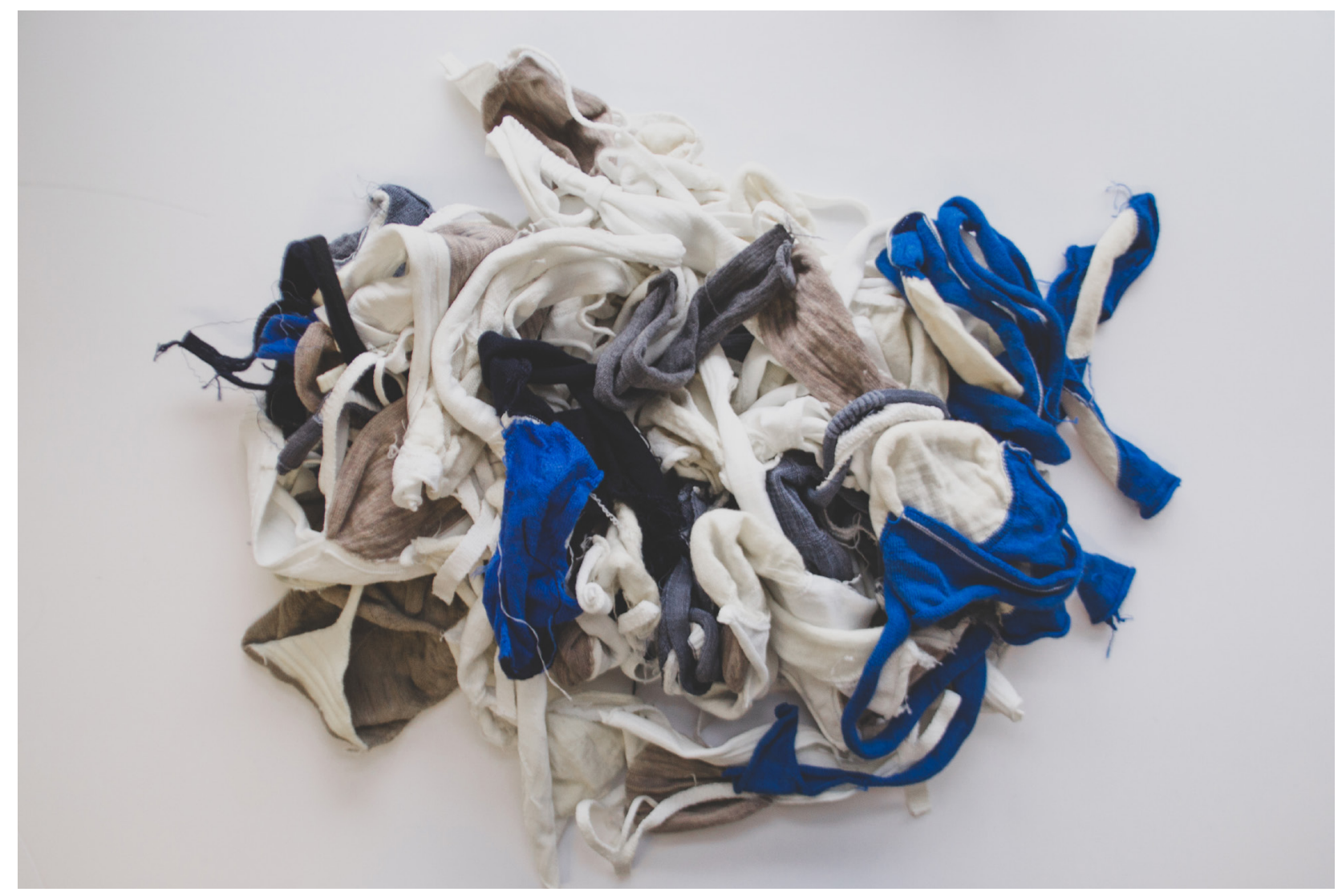




\section{Series I}
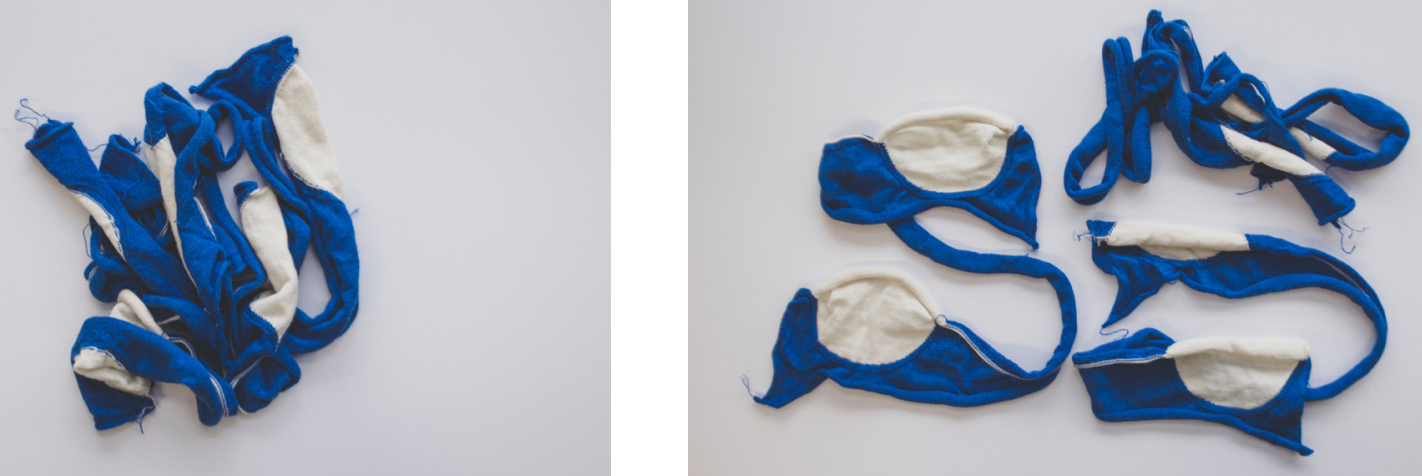

\section{Series II}
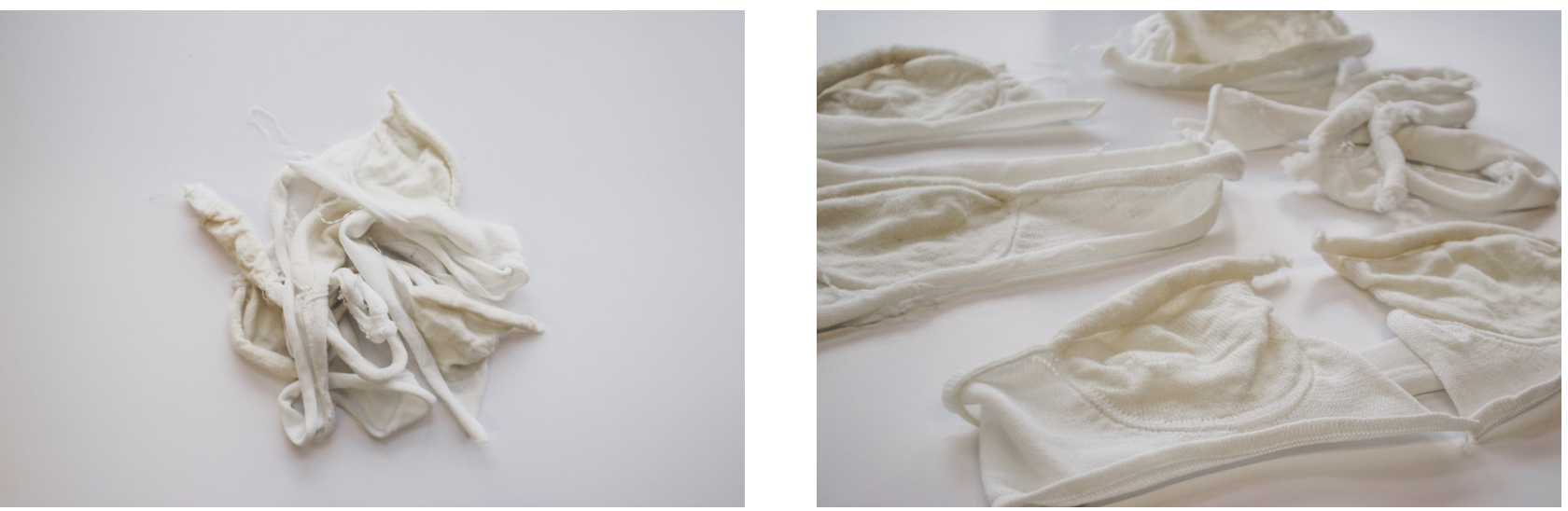

\section{Series III}
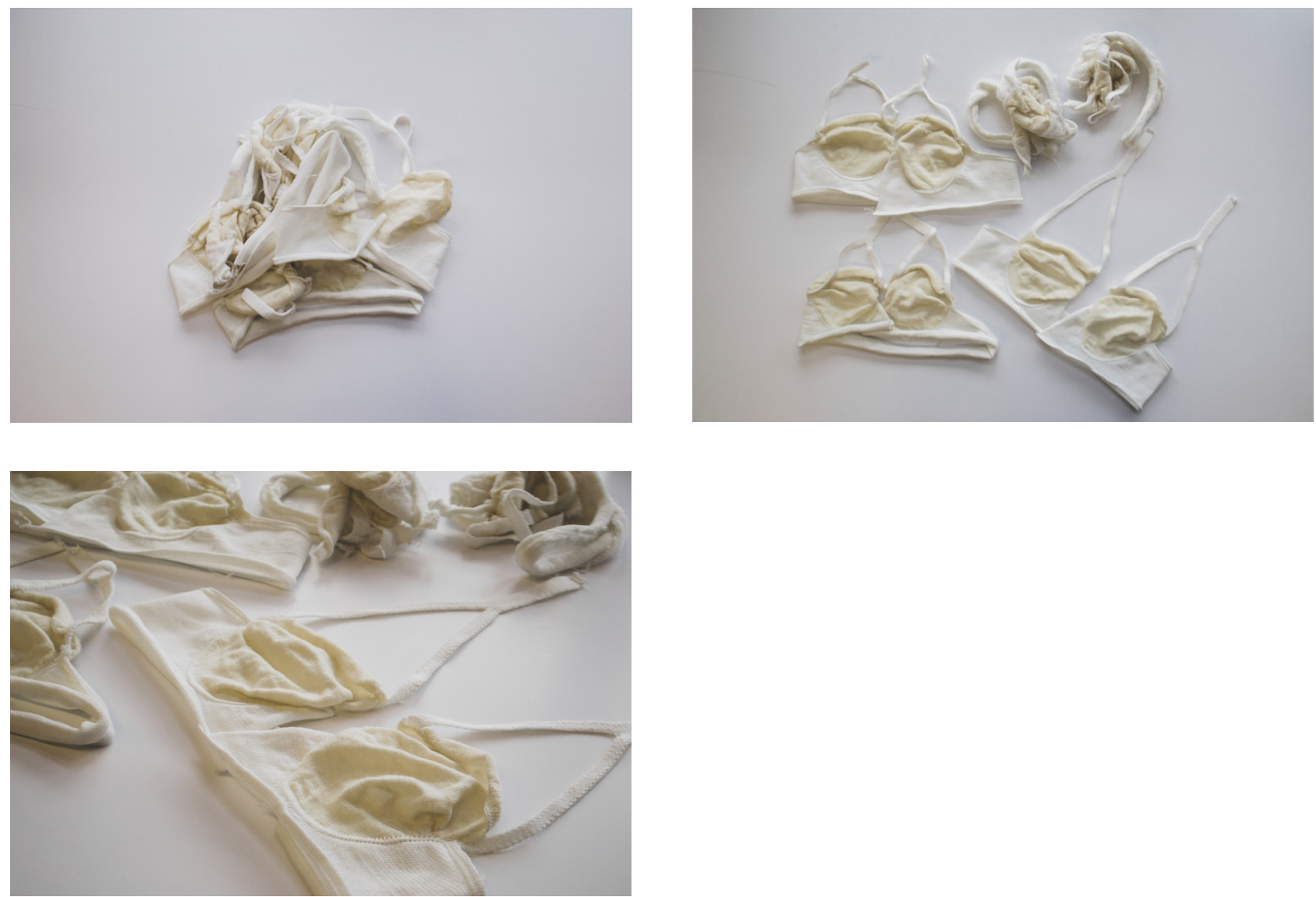
Series IV
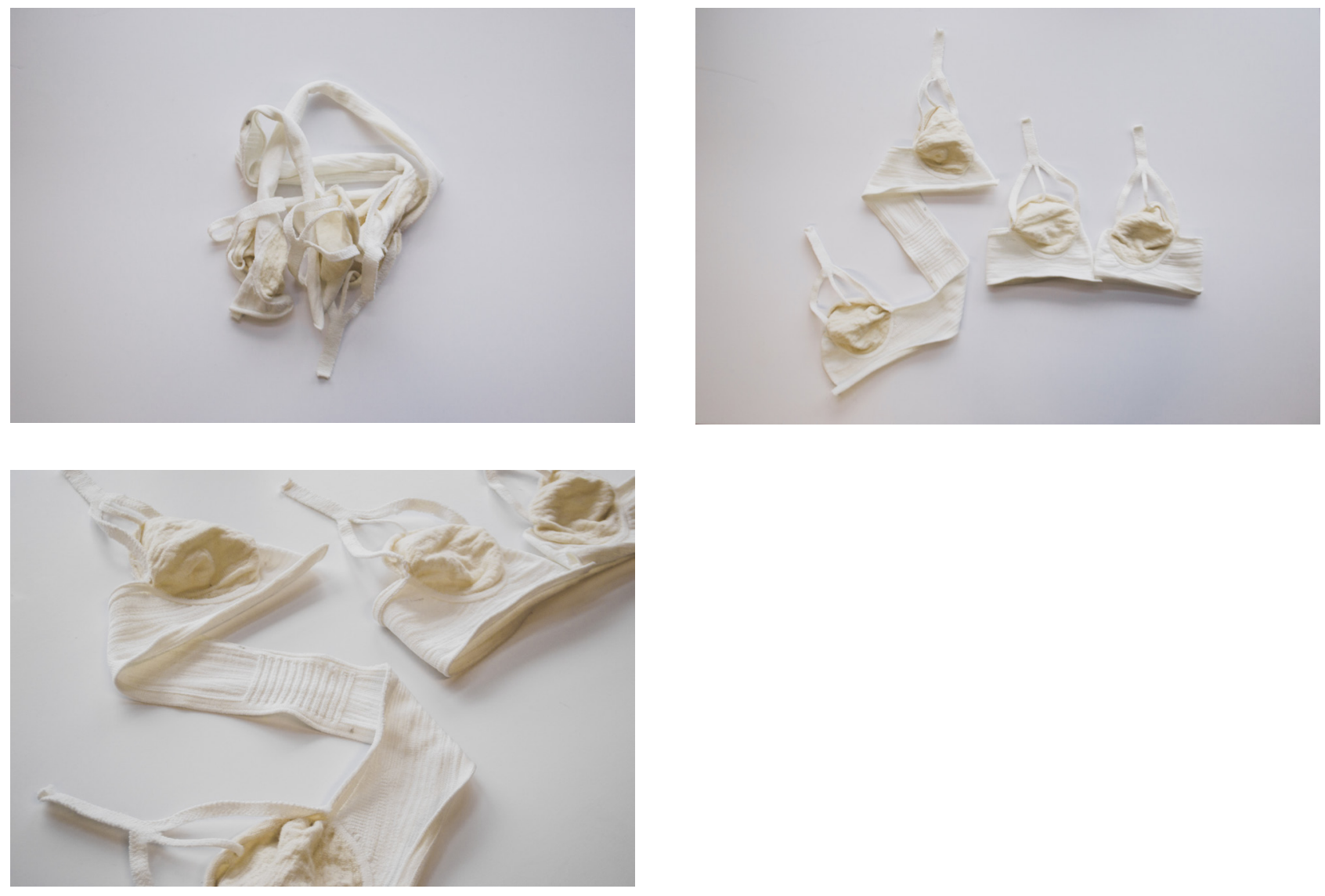

\section{Series V - VI}
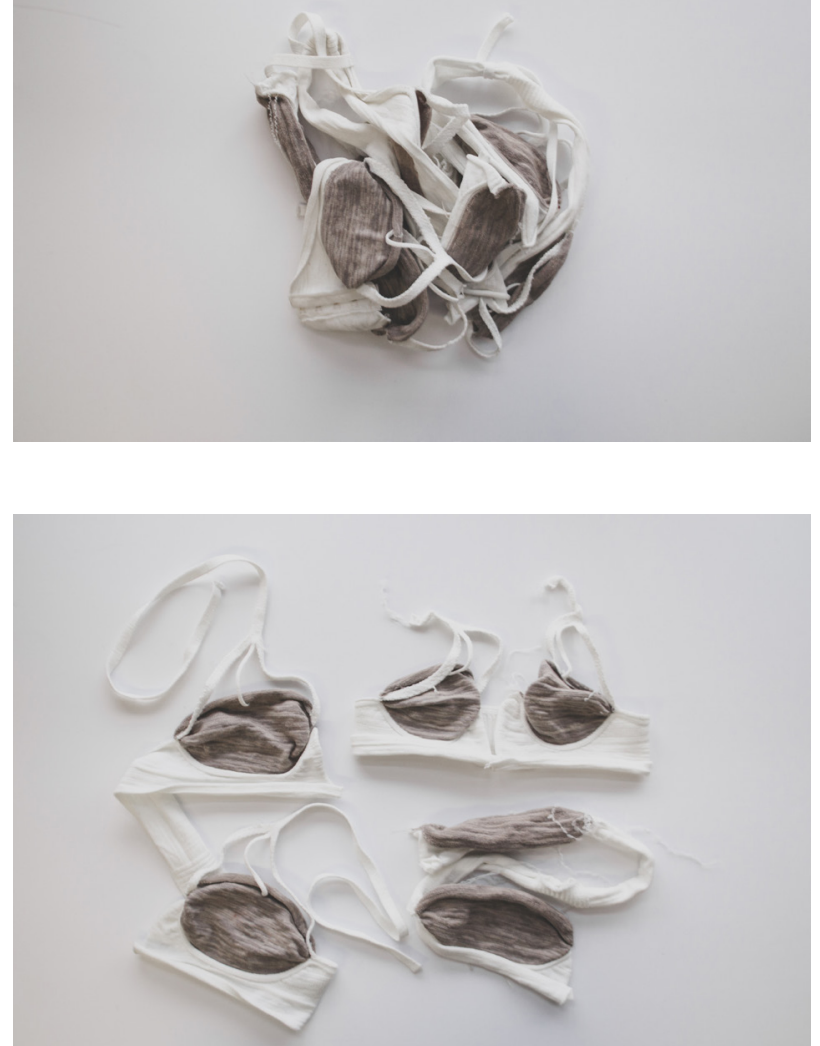

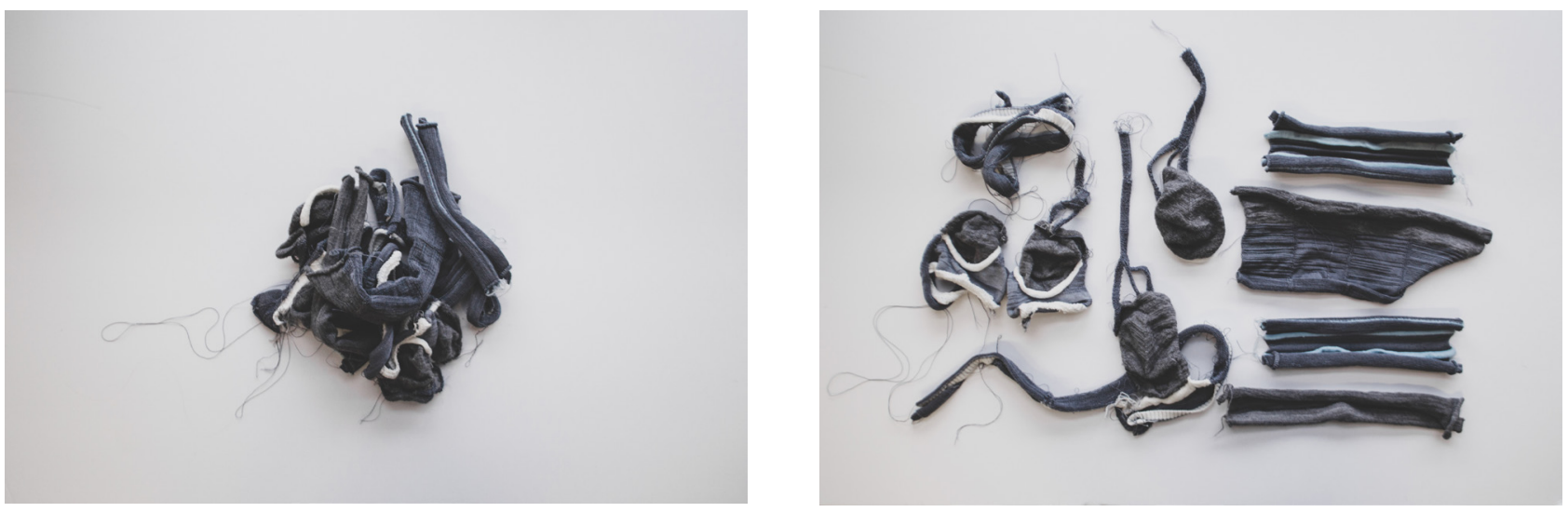

\section{Series VIII}
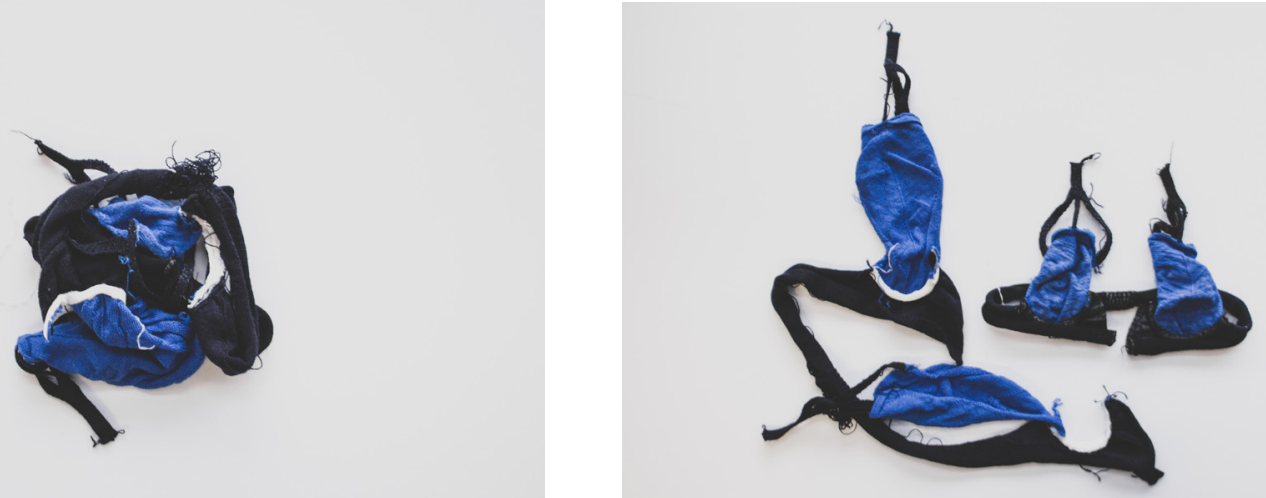

Series VIIII
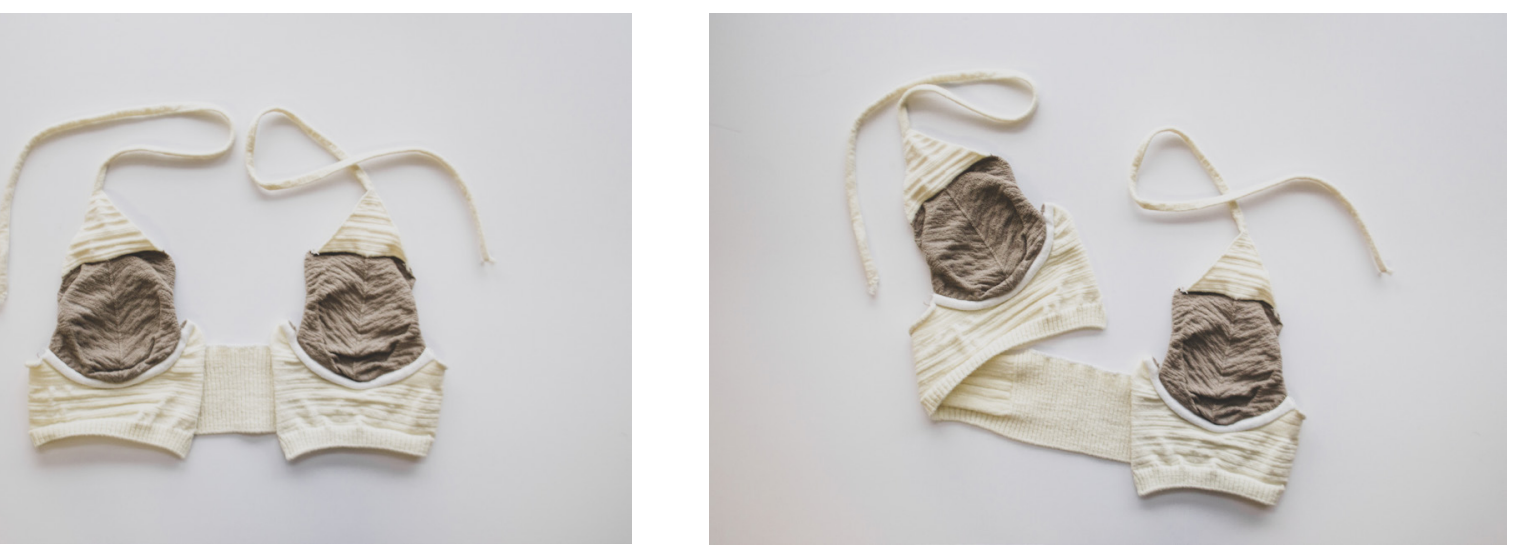
APPENDIX II CONTINUED

3D KNITTING DIGITAL PROGRAMMING 

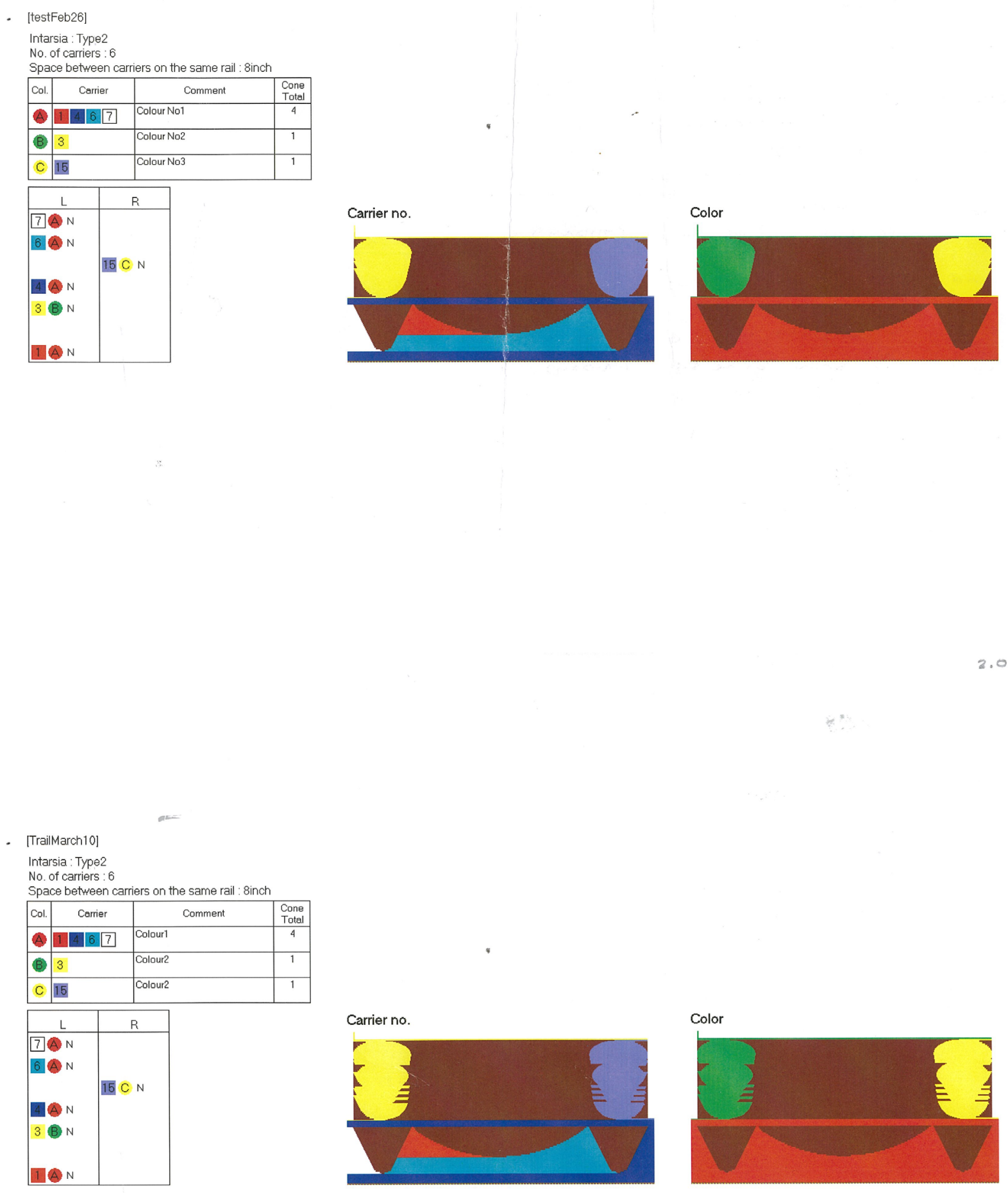

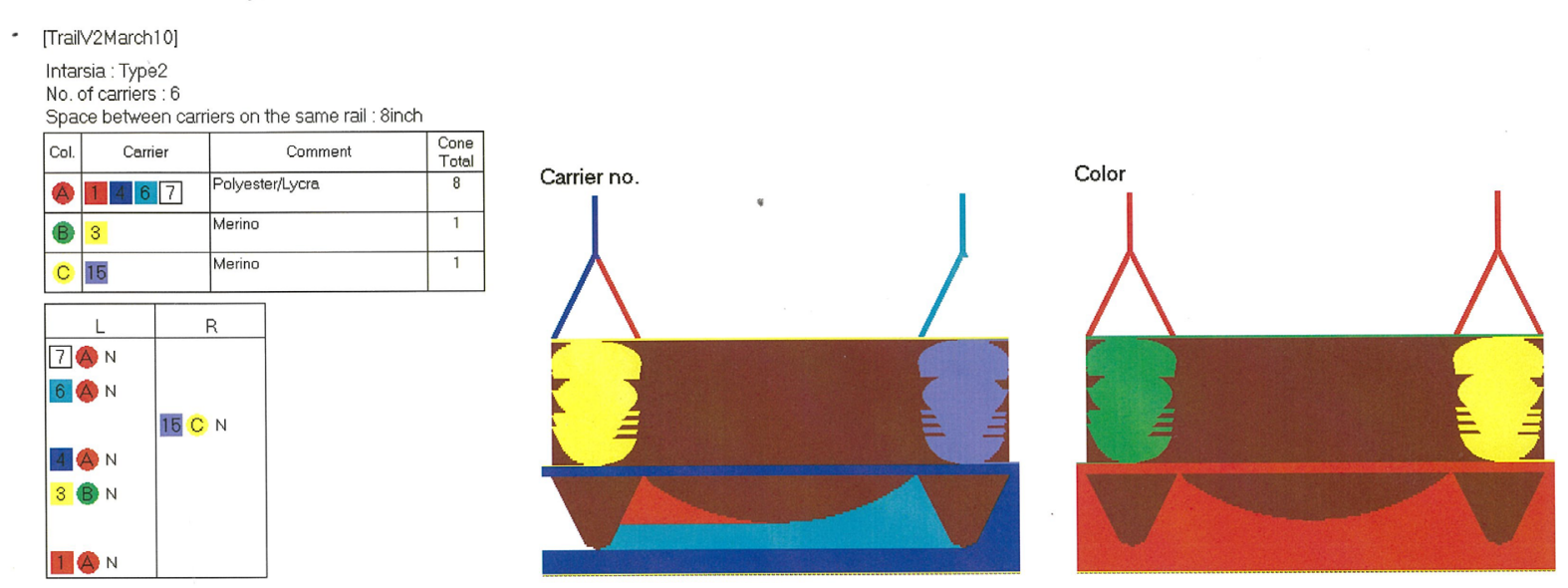

[TrailV4March11]

Intarsia : Type2

Space between carriers on the same rail : 8 inch

\begin{tabular}{|c|c|c|c|}
\hline Col. & Carrier & Comment & $\begin{array}{l}\text { Cone } \\
\text { Total }\end{array}$ \\
\hline (A) & 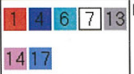 & Polyester/Lycra & 14 \\
\hline (B) & 315 & Merino/Lycra. & 2 \\
\hline
\end{tabular}

\begin{tabular}{|c|c|}
\hline L & $\mathrm{R}$ \\
\hline \multirow{3}{*}{$\begin{array}{l}7 \otimes \mathrm{N} \\
6 \otimes \mathrm{N}\end{array}$} & 17 A \\
\hline & \\
\hline & $15 \mathrm{~B} N$ \\
\hline $4 A \mathrm{~N}$ & $14 \otimes N$ \\
\hline $3 \mathrm{~N}$ & $13 \otimes N$ \\
\hline $1 A N$ & \\
\hline
\end{tabular}
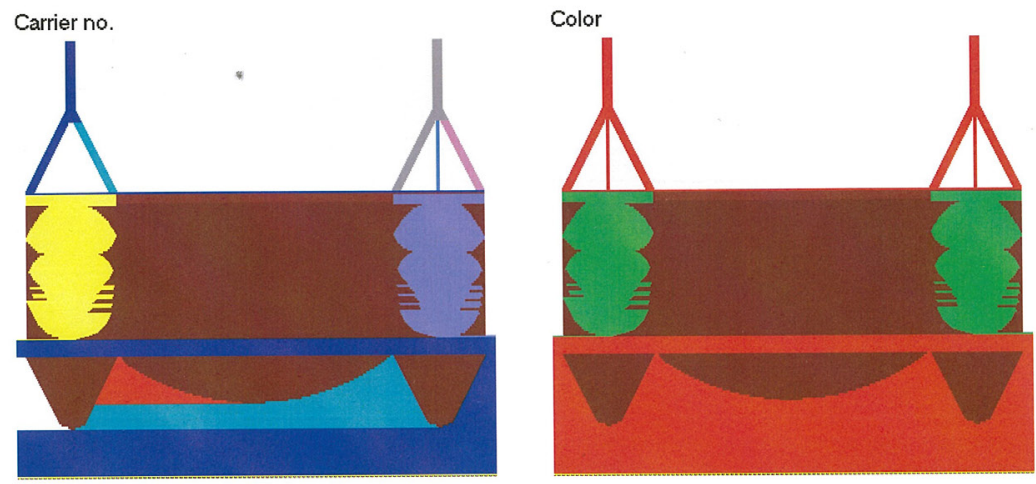
[Trial1April14

Intarsia : Type1

No of carriers: 11

Space between carriers on the same rail : 17.4inch

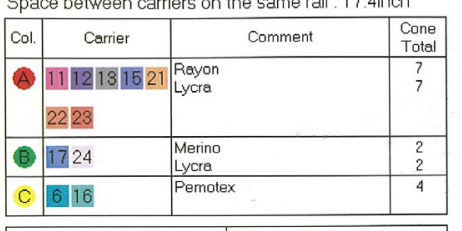

\begin{tabular}{|c|c|c|c|}
\hline \multicolumn{2}{|c|}{ L } & \multicolumn{2}{|c|}{$R$} \\
\hline Out & In & In & Out \\
\hline \multirow{7}{*}{$6 \mathrm{c} \mathrm{KSW}$} & 17 B KSW1 & & \\
\hline & $16 \mathrm{C} \mathrm{kSW}$ & & \\
\hline & 15 \& $\mathrm{KSW}$ & & \\
\hline & & 24 B KSW1 & \\
\hline & $13 \triangle \mathrm{KSW}$ & 23 KSW1 & \\
\hline & $128 \mathrm{KSW}$ & 22 ASW & \\
\hline & $11 \otimes \mathrm{KSW} 1$ & $21 \Leftrightarrow \mathrm{KSW}$ & \\
\hline
\end{tabular}
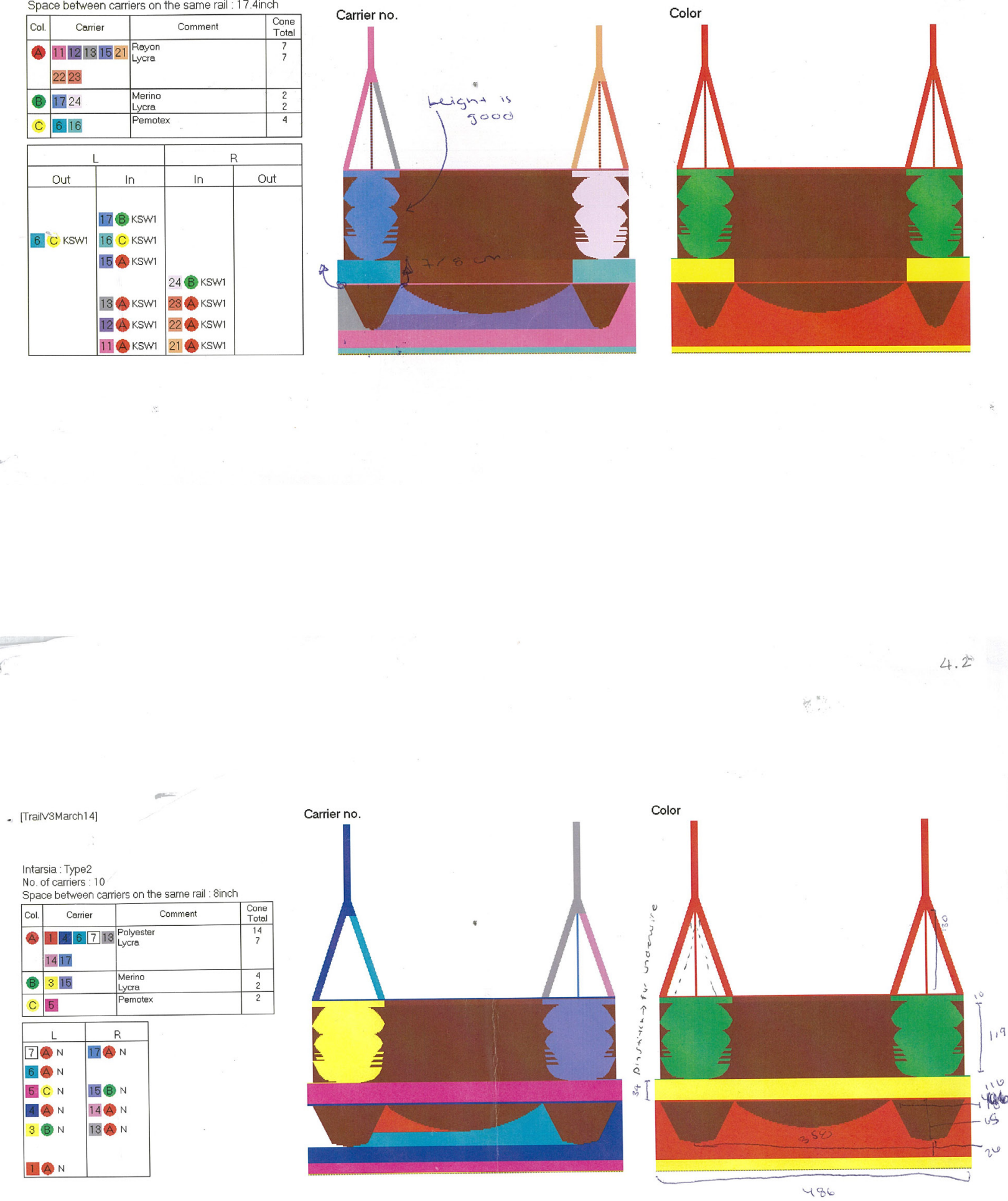


\section{APPENDIX III \\ ETHICS APPROVAL \\ INTERVIEW \& QUESTIONNAIRE SHEETS}

Victoria Univeristy of Wellington

Ethics Approval: Number 22243

Beyond girth and cup size: design of an adaptive bra system for better fit

18 December 2015 to 13 June 2016 


\section{CONVERSATION GUIDE}

Beyond girth and cup size: Design of an adaptive bra system for better fit

Researcher: Xuxu Amoozegar-Montero, School of Design, Victoria University of Wellington

\section{ATTITUDE TOWARDS BRAS}

1. Why do you wear a bra?

2. When do you wear a bra?

3. What style of bra do you prefer?

4. Are you satisfied with current bra designs?

5. What is a major problem in the design of bras?

6. What would you like to see in a new bra design?

7. Do you experience pain when wearing a bra? If so, where and when?

\section{BRA FITTING EXPERIENCE}

1. How likely are you able to find a bra that fits?

2. Was the bra you are currently wearing fitted by a fitting service? If so, by what kind of service? Professional bra fitter? Online fitting quiz? Or fitting phone app?

a. How often do you use a bra fitter? What kind of service?

b. Do different fitters help you each time?

c. If you have used more than one fitting method, are there noticeable differences between them?

d. Does the fitter find you the right fit?

3. How long does it take you to find a bra that fits?

4. How can you tell your bra is supportive?

Anything else about bra designs or your bra fitting experiences you would like to mention? 


\section{PARTICIPANT QUESTIONNAIRE}

Beyond girth and cup size: Design of an adaptive bra system for better fit

Researcher: Xuxu Amoozegar-Montero, School of Design, Victoria University of Wellington

Name:

Please answer the following questions to the best of your knowlegde. If you have any questions during this time please let me know.

\section{ABOUT YOUR BRA}

What size do you currently wear?

Do you think it fits you correctly? Circle one.

YES NO

How many hours do you normally wear a bra during the day?

How many bras do you own?

How many bras do you buy a year?

\section{BRA KNOWLEDGE}

Breasts are made up from:

Fat and glands,

Muscle and bone

Muscle and fat

Don't know

Muscle and glands 
SCHOOL OF DESIGN Te Kura Hoahoa

FACULTY OF ARCHITECTURE \& DESIGN Te Wāhanga Waihanga-Hoahoa

VICTORIA UNIVERSITY OF WELLINGTON, PO Box 600, Wellington 6140, New Zealand

Phone + 64-4-463 6200 Fax +64-4-463 6204 Email architecture@vuw.ac.nz Web www.victoria.ac.nz/design

Breasts are mainly supported by?

Skin

Muscle and bone

Coopers ligaments

Don't know

The bra size $10 \mathrm{C}$ is the same cup size (volume) as $14 \mathrm{C}$ ? Circle one.

YES NO

All bra sizes of $14 \mathrm{D}$ have the same underwire shape? Circle one.

YES NO

If $12 \mathrm{D}$ is too tight around the band, but cup is perfect, what would be the next appropriate size be?

$10 \mathrm{E}$

$14 \mathrm{C}$

$14 \mathrm{D}$

Don't know

The band of a new correctly fitted bra should be hooked to which hook?

Loosest

Tightest

Middle

Don't Know

\section{HEALTH AND BRAS}

Which factors influence discomfort? Tick all that apply.

$\begin{array}{llll}\square & \text { Fabric } & \square & \text { Cups } \\ \square & \text { Seam } & \square & \text { Hooks } \\ \square & \text { Underwire } & \square & \text { Padding } \\ \square & \text { Straps } & \square & \text { Other }\end{array}$

What is most important to you? Tick all that apply.

Comfort

Material

Support

Fit

Style

Quality

Price

Other 
